\title{
Aplicações de Approximate Bayesian Computation a controle de qualidade
}

\author{
Thiago Feitosa Campos
}

TESE APRESENTADA

$\mathrm{AO}$

Instituto De Matemática e Estatística

DA

Universidade DE SÃo PAUlo

PARA

OBTENÇÃO DO TÍTULO

$\mathrm{DE}$

DOUTOR EM CIÊNCIAS

\author{
Programa: Estatística \\ Orientador: Prof. Dr. Sergio Wechsler \\ Coorientador: Prof. Dr. Luís Gustavo Esteves
}

Durante o desenvolvimento deste trabalho o autor recebeu auxílio financeiro da CAPES

São Paulo, Junho de 2015 


\section{Aplicações de Approximate Bayesian Computation a controle de qualidade}

Esta versão da tese contém as correções e alterações sugeridas pela Comissão Julgadora durante a defesa da versão original do trabalho, realizada em 11/06/2015. Uma cópia da versão original está disponível no Instituto de Matemática e Estatística da Universidade de São Paulo.

Comissão Julgadora:

- Prof. Dr. Sergio Wechsler (orientador) - IME-USP

- Prof. Dr. Luís Gustavo Esteves - IME-USP

- Prof. Dr. Jesús Enrique García - UNICAMP

- Prof ${ }^{a}$. Dr ${ }^{\mathrm{a}}$. Laura Leticia Ramos Rifo - UNICAMP

- Prof. Dr. Marcio Alves Diniz - UFSCar 


\section{Agradecimentos}

A tarefa de escrever essa tese foi bastante difícil, ceratocone nos dois olhos Foi um fator complicador que tive que lidar durante a produção dessa tese. Não teria conseguido sem o apoio de amigos e família, em especial minha mãe que sempre deu apoio incondicional durante toda minha vida. Meus amigos Alexandre Patriota e Victor Fossaluza, atualmente professores do IME-USP, e Caio Freire foram importantes, sempre ajudando a manter a tempestade mental viva.

O período de tempo que passei como professor substituto na UFSCar foi basante engrandecedor para minha vida, e por isso quero agradecer a todos os professores e funcionários do departamento de estatística da universidade federal de São Carlos apenas por terem feito parte da minha vida naquele momento.

Minha amiga Raquel Guimarães e sua tia Catharina merecem um agradecimento especial por me ajudarem a superar momentos difíceis.

Ao meus amigos Alexander Strelow, e família, Fernando, Felipe(Pride), e família, e Léo, obrigado pelos momentos de descontração com o RPG, comidas gostosas e boa cerveja.

Alexandre Patriota e sua namorada, Fernanda, merecem um agradecimento adicional por terem me hospedado por uma semana, da ocasião da defesa da minha tese.

Desculpem aos meus amigos que esqueci de mencionar, mas agradeço a vocês por serem meus amigos. 


\section{Resumo}

CAMPOS, T. F. Aplicações de Approximate Bayesian Computation a controle de qualidade. 2015. 120 f. Tese (Doutorado) - Instituto de Matemática e Estatística, Universidade de São Paulo, São Paulo, 2015.

Neste trabalho apresentaremos dois problemas do contexto de controle estatístico da qualidade: monitoramento "on-line" de qualidade e enviromental stress screening, analisados pela óptica bayesiana. Apresentaremos os problemas dos modelos bayesianos relativos a sua aplicação e, os reanalisamos com o auxílio do $\boldsymbol{A} \boldsymbol{B} \boldsymbol{C}$ o que nos fornece resultados de uma maneira mais rápida, e assim possibilita análises diferenciadas e a previsão novas observações.

Palavras-chave: Approximate Bayesian Computarion, Controle de qualidade, Preditivismo. 


\section{Abstract}

CAMPOS, T. F. Applications of Approximate Bayesian Computation in quality control . 2015. 120 f. Tese (Doutorado) - Instituto de Matemática e Estatística, Universidade de São Paulo, São Paulo, 2015 .

In this work we will present two problems in the context of statistical quality control: on line quality monitoring and environmental stress screening, analyzed from the Bayesian perspective. We will present problems of the Bayesian models related to their application, and also we reanalyze the problems with the assistance of $\boldsymbol{A} \boldsymbol{B C}$ methods which provides results in a faster way, and so enabling differentiated analyzes and new observations forecast.

Keywords: Approximate Bayesian Computation, Quality control, Forecast. 


\section{Sumário}

$\begin{array}{ll}\text { Lista de Figuras } & \text { ix }\end{array}$

Lista de Tabelas $\quad$ xi

$\mathbf{1}$ Introdução $\quad \mathbf{1}$

1.1 Organização do trabalho . . . . . . . . . . . . . . . . . . . . . . . 2

2 Approximate Bayesian Computation $\quad 5$

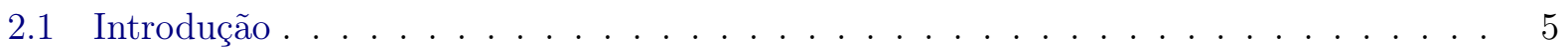

2.2 Função de verossimilhança . . . . . . . . . . . . . . . . . . 5

2.3 Algoritmo $A B C \ldots \ldots \ldots \ldots \ldots \ldots$

2.3.1 Gênese do Approximate Bayesian Computation . . . . . . . . . . . . 7

2.3.2 Observando variáveis aleatórias contínuas . . . . . . . . . . . . 8

$2.3 .3 \quad M C M C-A B C \ldots \ldots \ldots \ldots \ldots$

2.3 .4 Noisy $A B C \ldots \ldots \ldots \ldots \ldots \ldots$

2.4 Toy model . . . . . . . . . . . . . . . . . . . . . . . . . . . . 11

2.4 .1 Beta-binomial . . . . . . . . . . . . . . . . . 11

$2.4 .2 \quad$ Normal-gama . . . . . . . . . . . . . . . . . . . . . . 13

2.5 Comparando o $\boldsymbol{A B C}$ com um algoritmo $M C M C \ldots \ldots \ldots \ldots$

3 Gerando observações de uma distribuição de Holgate $\quad 21$

3.1 Introdução . . . . . . . . . . . . . . . . . . . . . . . . . . . . 21

3.2 Distribuição de Poisson bivariada . . . . . . . . . . . . . . . . . . . . 21

3.3 Abordagem Bayesiana . . . . . . . . . . . . . . . . . . . . . . 23

3.4 Gerando observações . . . . . . . . . . . . . . . . . . . . . . . . . 24

3.4.1 Gibbs Sampler . . . . . . . . . . . . . . . . . . . . 24

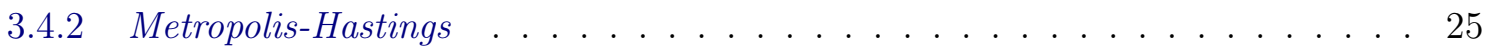

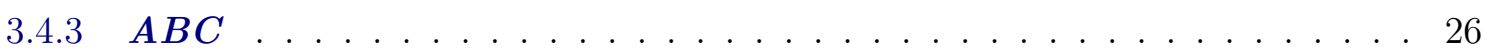

3.4 .4 Comparando métodos . . . . . . . . . . . . . . . . . . . 28

3.5 Prevendo novas observações $\ldots \ldots \ldots \ldots \ldots \ldots$

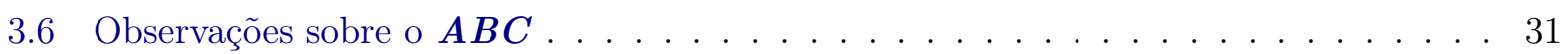

$4 A B C$ aplicado ao modelo Bayesiano não-paramétrico para o monitoramento "online" de qualidade de Taguchi para atributos. $\quad 45$

4.1 Introdução . . . . . . . . . . . . . . . . . . . . . . . . . . 45

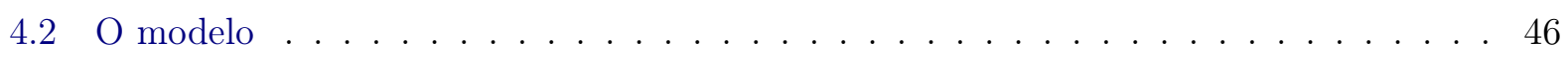

4.2 .1 Suposições . . . . . . . . . . . . . . . . . . . . . . 46 
4.2 .2 Modelo . . . . . . . . . . . . . . . . . . . . . . . 46

4.2 .3 Vantagens e desvantagens . . . . . . . . . . . . . . . . 47

$4.3 A B C$ aplicado ao modelo não paramétrico . . . . . . . . . . . . . . . . . . . 48

4.3.1 Gerando observações de um processo de Dirichlet . . . . . . . . . . . . . . . . 48

4.3.2 Gerando observações do processo de controle de qualidade. . . . . . . . . . . . 52

4.3.3 $\boldsymbol{A B C}$ configurado para gerar uma função de probabilidade acumulada . . . . 52

4.3.4 Escolha do método para gerar uma observação de um processo de Dirichlet . 54

4.4 Calculando a estimativa da proporção dos itens não-conforme ao longo do processo de produção usando o amostrador de Gibbs . . . . . . . . . . . . . . . . . . . . . . 54

4.5 Resultados das simulações do $\boldsymbol{A B C} \ldots \ldots \ldots \ldots \ldots \ldots$

4.5.1 Análise dos hiperâmetros . . . . . . . . . . . . . . . 56

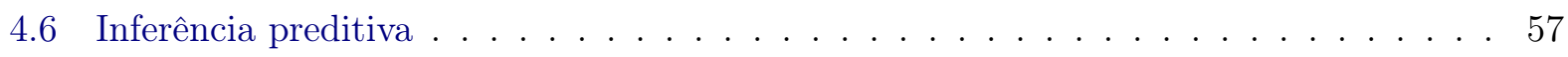

4.7 Observações e considerações finais . . . . . . . . . . . . . . . 57

$5 \quad A B C$ aplicado a Enviromental Stress Screening $\quad 67$

5.1 Introdução . . . . . . . . . . . . . . . . . . . . 67

5.2 Abordagem Bayesiana para a metodologia $E S S \ldots \ldots \ldots$. . . . . . . . . . 67

5.2 .1 Função de risco a priori . . . . . . . . . . . . . . . . 68

5.2 .2 Análise Bayesiana . . . . . . . . . . . . . . . . . . . . . 68

5.3 Usando o $\boldsymbol{A B C}$ na metodologia $\boldsymbol{E S S} \ldots \ldots \ldots \ldots \ldots \ldots$

5.3 .1 Exemplo numérico . . . . . . . . . . . . . . . . . . . . 70

$5.4 \quad M C M C$ aplicado à metodologia $E S S \ldots \ldots \ldots \ldots \ldots \ldots$

5.5 Taxa de fala crescente . . . . . . . . . . . . . . . . . . . . 73

5.5.1 Modelo com taxa de falha crescente . . . . . . . . . . . . . . . . 74

5.5 .2 Exemplo numérico . . . . . . . . . . . . . . . . . . . . 76

5.6 Previsões de futuros enviromental stress screening . . . . . . . . . . . . . . . . 77

5.7 Considerações finais . . . . . . . . . . . . . . . . . . . . . . 77

6 Conclusões $\quad 87$

6.1 Sugestões para Pesquisas Futuras . . . . . . . . . . . . . . . . . . 88

$\begin{array}{lr}\text { Referências Bibliográficas } & 89\end{array}$ 


\section{Lista de Figuras}

2.1 (a) Histograma da amostra média gerada no primeiro cenário comparada com a densidade a posteriori (equação 2.12); (b) Histograma da amostra média gerada no segundo cenário comparada com a densidade a posteriori (equação 2.12); (c) Histograma da amostra média gerada no terceiro cenário comparada com a densidade a posteriori (equação 2.12); (d) Histograma da amostra média gerada no quarto cenário comparada com a densidade a posteriori (equação 2.12 ) . . . . . . . . . 16

2.2 (a) Função de probabilidade acumulada de $(\mu, \tau) \mid \mathbf{x}$; (b) Função empírica média da amostra do primeiro cenário; (c) Função empírica média da amostra do segundo cenário. 17

2.3 (a) Gráfico de dispersão de todas as amostras geradas no primeiro cenário; (b) Gráfico de dispersão de todas as amostras geradas no segundo cenário. . . . . . . . . . . 18

2.4 (a) Função de probabilidade acumulada de $(\mu, \tau) \mid \mathbf{x}$; (b) Função acumulada empírica média da amostra do primeiro cenário; (c) Função acumulada empírica média da amostra do segundo cenário; (d) Função acumulada empírica média da amostra gerada pelo amostrador de Gibbs. . . . . . . . . . . . . . . . . . . 19

2.5 (a) Gráfico de dispersão de todas as amostras geradas no primeiro cenário; (b) Gráfico de dispersão de todas as amostras geradas no segundo cenário; (c) Gráfico de dispersão de todas as amostras geradas pelo amostrador de Gibbs. . . . . . . . . . . . 20

3.1 Boxplot do número da iterações que cada algoritmo necessitou para gerar a amostra. 33

3.2 Histogramas de observações a posteriori de $\lambda_{1}, \lambda_{2}$ e $\lambda_{3}$ geradas pelo $M C M C$ via

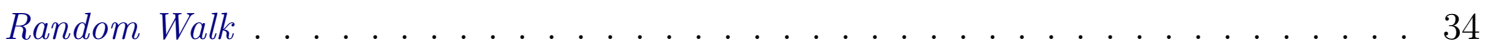

3.3 Histogramas de observações a posteriori de $\lambda_{1}, \lambda_{2}$ e $\lambda_{3}$ geradas pelo $M C M C$ via Independence Chain . . . . . . . . . . . . . . . . . . . . . . . 35

3.4 Histogramas de observações, geradas pelo $\boldsymbol{A} \boldsymbol{B} \boldsymbol{C}$ a posteriori de $\lambda_{1}, \lambda_{2}$ e $\lambda_{3} \ldots \ldots 6$

3.5 Histograma em três dimensões das amostras de $\lambda_{1}$ versus $\lambda_{2}$ geradas pelo Independence Chain MCMC . . . . . . . . . . . . . . . . . . . . . 37

3.6 Histograma em três dimensões das amostras de $\lambda_{1}$ versus $\lambda_{3}$ geradas pelo Indepen-

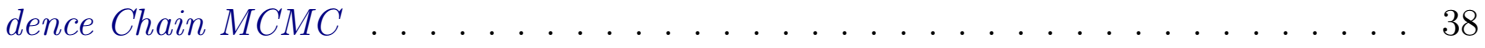

3.7 Histograma em três dimensões das amostras de $\lambda_{3}$ versus $\lambda_{3}$ geradas pelo Independence Chain MCMC . . . . . . . . . . . . . . . . . . . . . . . 39

3.8 Histograma em três dimensões das amostras de $\lambda_{1}$ versus $\lambda_{2}$ geradas pelo $\boldsymbol{A B C}$. . 40

3.9 Histograma em três dimensões das amostras de $\lambda_{1}$ versus $\lambda_{3}$ geradas pelo $\boldsymbol{A} \boldsymbol{B} \boldsymbol{C}$. . 41

3.10 Histograma em três dimensões das amostras de $\lambda_{3}$ versus $\lambda_{3}$ geradas pelo $\boldsymbol{A} \boldsymbol{B} \boldsymbol{C}$. . . 42

3.11 Disgrama de dispersão em três dimensões da distribuição preditiva . . . . . . . . . . 43

3.12 Representação da distribuição preditiva na forma de um histograma em três dimensões 44 
4.1 Número de linhas da matriz $\mathbb{C}_{k} \ldots \ldots \ldots \ldots$. . . . . . . . . . . . . . 49

4.2 Número de linhas da matriz $\mathbb{C}_{k}^{\prime} \ldots \ldots \ldots \ldots \ldots$. . . . . . . . . . . . . 50

4.3 Boxplot do numero de interações necessárias para gerar amostras utilizando o stickbreacker e o Rapid Simulation, como amostradores da distribuição priori . . . . . . . 55

4.4 Estimativas de $F(t) \mid \tau=k$ geradas pelo $\boldsymbol{A B C}$, usando o Stickbreaker e o Rapid Simulations of A Dirichlet Process . . . . . . . . . . . . . . . . . . . 59

4.5 Estimativas de $F(t) \mid \tau=1$, obtidos pela equação 4.4, pelo $\boldsymbol{A B C}$ (algoritmo 14) e

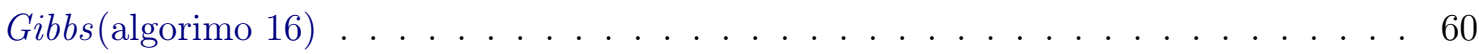

4.6 Estimativas de $F(t) \mid \tau=k$ para $a=b=\gamma=\Lambda=\Delta=5$, dado $\tau=k$ para $k=1, \ldots, 1061$

4.7 Estimativas de $F(t) \mid \tau=1$ variando de acordo com valores de $a \ldots \ldots$. . . . . 62

4.8 Estimativas de $F(t) \mid \tau=1$ variando de acordo com valores de $b \ldots \ldots$. . . . . . . 63

4.9 Estimativas de $F(t) \mid \tau=1$ variando de acordo com valores de $\gamma \ldots \ldots$. . . . . . 64

4.10 Estimativas de $F(t) \mid \tau=1$ variando de acordo com valores de $\Lambda \ldots \ldots$. . . . . . 65

4.11 Estimativas de $F(t) \mid \tau=1$ variando de acordo com valores de $\Delta \ldots \ldots 6$

5.1 Histograma das amostras da distribuição de probabilidade a posteriori de $p, \lambda_{e}$ e $\lambda_{g}$ geradas pelo algoritmo $18 \ldots \ldots \ldots \ldots \ldots$

5.2 Função de risco a posteriori . . . . . . . . . . . . . . . . . 80

5.3 Histograma das amostras de $\left(\alpha, p, \lambda_{g}, \lambda_{e}\right)$ gerados pelo $M C M C$, algoritmo $19 \ldots 1$

5.4 Função de risco a priori quando a taxa de falha é crescente . . . . . . . . . . . . . 82

5.5 Histograma das amostras da distribuição a posteriori de $\left(\alpha, p, \lambda_{g}, \lambda_{e}, k_{g}, k_{e}\right) \ldots \ldots$

5.6 Função de risco a posteriori . . . . . . . . . . . . . . . . . . 84

5.7 Gráfico de barras das distribuições preditivas marginais de $x, y, z$ e $m$ suponto taxa de falha constante. . . . . . . . . . . . . . . . . 85

5.8 Gráfico de barras das distribuições preditivas marginais de $x, y, z$ e $m$ suponto taxa de falha crescente. . . . . . . . . . . . . . . . . 86 


\section{Lista de Tabelas}

2.1 Sumário do número de interações necessárias para gerar uma observação usando o amostrador de Gibbs . . . . . . . . . . . . . . . . . . . . 15

3.1 Sumário do número de iterações necessárias para gerar uma observação da distribuição de Holgate usando os algoritmos 9,10 e $11 \ldots$. . . . . . . . . . . . . 29

3.2 Distribuição preditiva . . . . . . . . . . . . . . . . . . . . . 31

4.1 Estatísticas descritivas do número de interações que o $\boldsymbol{A B C}$ levou para gerar as estimativas de $F(t) \mid \tau=k$, usando tanto o Stickbreaker como o Rapid Simulation . . 54

4.2 Distribuição preditiva do número de inspeções necessárias para detectar um item não-conforme, num próximo ciclo de produção . . . . . . . . . . . . . . 58 


\section{Lista de Algoritmos}

$1 \quad A B C$-Rejeição $1 \ldots \ldots \ldots \ldots \ldots \ldots$

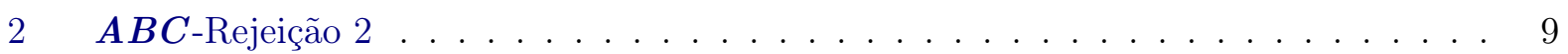

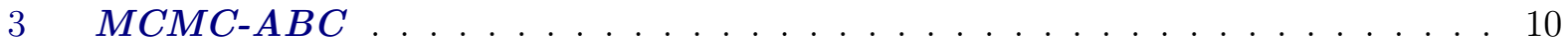

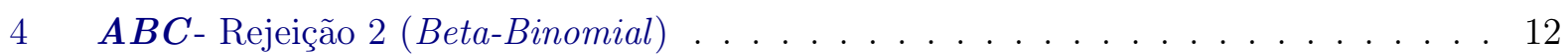

$5 \quad \boldsymbol{A} \boldsymbol{B C}$ - Rejeição $2($ Normal-gama $\ldots \ldots \ldots \ldots \ldots \ldots \ldots$

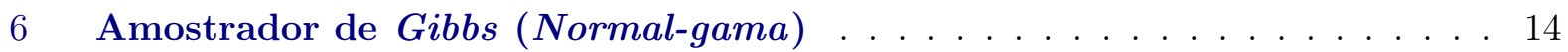

7 Amostrador de Gibbs para gerar observações da distribuição Holgate . . . . . . . . . 25

8 Metropolis-Hastings . . . . . . . . . . . . . . . . . . . 25

9 Random Walk Chain Metropolis-Hastings . . . . . . . . . . . . . . . . . 27

10 Independence Chain Metropolis-Hastings . . . . . . . . . . . . . . . . . . . . . 27

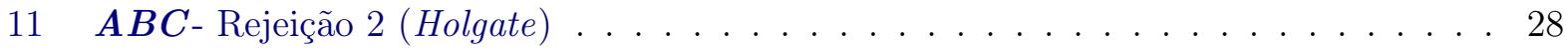

12 Gerador de processo de Dirichlet . . . . . . . . . . . . . . . . . . . . . . 52

13 Gerador de processo de controle de qualidade de Taguchi . . . . . . . . . . . . . . 52

14 Processo de estimação de $F(t) \mid \tau=k \ldots \ldots \ldots \ldots \ldots$

15 Amostrador de Gibbs para obter o estimador bayesiano não-paramétrico da função de sobrevivência para o caso de censura intervalar quando a priori é processo de Dirichlet. . . . . . . . . . . . . . . . . . . 56

16 amostrador de Gibbs para obter observações da distribuição 4.4 . . . . . . . . . . . 56

$17 \boldsymbol{A B C}$ para gerar amostras de $\pi\left(\alpha, p, \lambda_{g}, \lambda_{e} \mid x, y, z, m\right) \ldots \ldots \ldots \ldots$

$18 \boldsymbol{A B C}$ para gerar amostras de $\pi\left(\alpha, p, \lambda_{g}, \lambda_{e} \mid x, y, z, m\right) \ldots \ldots \ldots \ldots \ldots$

19 MCMC via independence chain para gerar amostras do modelo $5.8 \ldots \ldots$. . . . . 73

$20 \boldsymbol{A B C}$ para gerar amostras de $\pi\left(\alpha, p, \lambda_{g}, \lambda_{e}, k_{g}, k_{e} \mid x, y, z, m\right) \ldots \ldots \ldots$ 


\section{Capítulo 1}

\section{Introdução}

Os fenômenos da natureza sempre chamaram atenção do homem. Na pré-historia o conhecimento sobre os fenômenos naturais representava a diferença entre viver ou morrer. A capacidade de poder explicar, e com isso poder prever tais fenômenos se tornou importante para a evolução humana. Tal cenário demonstra o importante papel que um esforço de explicação e previsão teve em termos práticos tanto quanto em termos explicativos

Com a necessidade de obter novas explicações sobre o que era contemplado pelo ser humano, sugiram novas formas de responder aos questionamentos levantados perante tais fenômenos. O pensamento matemático tornou-se uma alternativa para explicar tais contemplações. Por considerar a aleatoriedade, caos dos fenômenos observados, a abordagem estatística é a forma mais adaptada tal tarefa.

Modelos estatísticos vem evoluindo de maneira similar às explicações dos fenômenos observados. Essa evolução do modelo deve-se, em grande parte, a evolução tecnológica. Atualmente, modelos com suposições cada vez mais sofisticadas podem ser criados, avaliados e usados no mundo prático. Dos modelos, mais especificamente modelos estatísticos, possuidores de sofisticações teóricas, que aumentam o nível de complexidade do mesmo, os que mais se beneficiaram do avanço tecnológico são os modelos sob a óptica Bayesiana. Um exemplo são modelos que não tinham um teorema que estipulava uma família conjugada e, com o avanço tecnológico, passaram a ser frequentes.

Com a constante sofisticação dos modelos, desejo latente de alguns estatísticos, um fenômeno aflorou, a extrema complexidade computacional dos mesmos. Até que ponto a contínua sofisticação de modelos estatísticos é possível, levando em consideração a complexidade gerada por ela? Quais custos a utilização de um modelo super sofisticado pode trazer ao seu usuário? Quais os ganhos e perdas tem-se por utilizar um modelo menos sofisticado, mas mais acessível?

O controle estatístico do processo de produção de um determinado item é uma área de estudo em que a complexidade de modelos estatística é uma característica indesejável. Quanto maior a complexidade do modelo utilizado maior é o tempo necessário para diagnosticar uma possível quebra na qualidade do processo de produção.

Assegurar o controle de qualidade de uma linha de produção é essencial para evitar a comercialização de produtos defeituosos. Tsunemi et al. (2012) e Barlow et al. (1994) propõem modelos Bayesianos para dois procedimentos de monitoramento da qualidade do processo de produção. $\mathrm{O}$ primeiro trata o monitoramento de um processo de produção em linha segundo a abordagem de Taguchi (ver Taguchi et al. (1989)). Tsunemi et al. (2012) apresentam uma abordagem Bayesiana não-paramétrica para modelar a verificação de um item não-conforme durante uma inspeção do ciclo de produção.

Já em Barlow et al. (1994) o contexto abordado é o da pós-produção. É assumido que os itens produzidos podem atender ou não a qualidade estipulada. Um lote destes itens é submetido a um ensaio de estresse simulando, de forma acelerada, o tempo de uso. O trabalho em questão apresenta uma abordagem bayesiana para estimar a proporção de itens de baixa qualidade que foram produzidos, bem como o tempo médio da vida útil dos itens de boa e baixa qualidade.

Em ambos os modelos há um fato em comum, uma complexidade computacional intensa, ao ponto de inviabilizar o seu uso. Tal complexidade é devida a suposições teóricas extremamente sofisticadas, feitas nos seus respectivos trabalhos. 
Quando um modelo em estudo é complicado de analisar, pelo fato de não ter uma forma analítica amigável, a opção para a utilização do mesmo, é a geração de observações deste modelo em estudo.

Existem diversos métodos para gerar observações de um modelo estatístico, todos baseados na distribuição de probabilidade associada ao modelo. O mais simples é utilizar a função inversa da distribuição acumulada $\left(F^{-1}\right)$.

Quando a complexidade envolvida na análise é grande, são necessários métodos computacionais mais potentes. Para os modelos Bayesianos mais sofisticados os métodos mais usuais são baseados em Markov Chain Monte Carlo, MCMC. O amostrador de Gibbs e o algoritmo de MetropolisHastings são os algoritmos mais utilizados.

O lado negativo dessa estratégia de análise é o fato de que esses algoritmos dependem direta, ou indiretamente, da forma da função de verossimilhança. Dependendo da complexidade da função de verossimilhança, como é o caso do modelo apresentado por Tsunemi et al. (2012), gerar observações do modelo continua sendo uma tarefa extremamente difícil. Os modelos apresentados em Tsunemi et al. (2012) e Barlow et al. (1994) são dois modelos que apresentam uma complexidade computacional tão grande que impossibilita até mesmo a utilização de métodos $M C M C$, como será visto e discutido mais a frente nesse trabalho.

Em casos como esse que o Approximate Bayesian Computation $(\boldsymbol{A B C})$ se destaca. Em sua essência está o fato de não utilizar, de forma numérica, a função de verossimilhança. Essa premissa é o grande atrativo do $\boldsymbol{A B C}$ e, também, um ponto de grande discussão. Pela sua sua ideia motriz, o $\boldsymbol{A B C}$ é o candidato ideal para gerar observações dos modelos supracitados.

O $\boldsymbol{A} \boldsymbol{B} \boldsymbol{C}$ é bastante usado em áreas biológicas: Beaumont et al. (2002) implementam o método em genética de populações e discutem o problema de inúmeros parâmetros indesejados nos modelos estatísticos utilizados nessa área de pesquisa. Em Cornuet et al. (2008) é proposto um programa computacional, Do It Yourself Approximate Bayesian Computation (DIY ABC) para analisar dados de população genética e aferir informações sobre a história evolutiva da mesma. Beaumont (2010) descreve o $\boldsymbol{A} \boldsymbol{B} \boldsymbol{C}$, bem como técnicas de $\boldsymbol{M C M C}$ nos contextos de populações genéticas, demografia de colonização, história demográfica humana, filogeografia, entre outros.

No presente trabalho é introduzido o uso do $\boldsymbol{A} \boldsymbol{B} \boldsymbol{C}$ no contexto de controle de qualidade. Com isso é esperado que as análises dos modelos de Tsunemi et al. (2012) e Barlow et al. (1994) fiquem mais palpáveis e, assim, fazer com eles sejam utilizados com mais frequência . Outra ambição é a possibilidade estender os resultados, já conhecidos, para outros casos igualmente interessantes. Por exemplo, relaxar a suposição de taxa de falha constante, presente em Barlow et al. (1994), usando uma taxa de falha crescente.

Com o objetivo de manter a complexidade computacional no nível mais baixo possível, decidiu-se por apenas implementar a versão mais simples do $\boldsymbol{A B C}$.

\subsection{Organização do trabalho}

Esse trabalho se organiza da seguinte forma. O capítulo 2 apresenta o algoritmo Approximate Bayesian Computation em seus detalhes. Discute-se o fato do $\boldsymbol{A B C}$ usar, ou não, a função de verossimilhança de um modelo. O funcionamento é ilustrado através de toy models. Suas peculiaridades são discutidas, o que muda nas amostras geradas quando mudamos os argumentos computacionais do $\boldsymbol{A} \boldsymbol{B} \boldsymbol{C}$ e, por fim, o desempenho do $\boldsymbol{A} \boldsymbol{B} \boldsymbol{C}$ é comparado com o do $\boldsymbol{M C M C}$.

No capítulo 3 é apresentado um breve resumo sobre a distribuição de Holgate, que possui uma função de probabilidade, e por consequência uma função de verossimilhança, analiticamente complicada. O $\boldsymbol{A B C}$, e o $\boldsymbol{M C M C}$, são configurados para gerar observações de uma distribuição de probabilidade a posteriori, distribuição dos dados observados é a distribuição Holgate, e seus desempenhos comparados. Acredita-se que esse pode ser um exemplo esclarecedor de que o $\boldsymbol{A} \boldsymbol{B} \boldsymbol{C}$ pode gerar amostras de um modelo sem necessitar de sua função de verossimilhança.

Nos capítulos 4 e 5 é seguido o seguinte roteiro, para ambos os capítulos. Apresentar o problema em estudo, revisando os artigos de origem do problema, justificar o uso do $\boldsymbol{A} \boldsymbol{B C}$, aplicar o mesmo, compara-lo com o $M C M C$ e, por fim usar os dados gerados para obter a distribuição de probabilidade preditiva de um nova observação do experimento de interesse. 
Finalmente, no capítulo 6, conclui-se o trabalho, e são apresentadas diretrizes para futuros trabalhos. 


\section{Capítulo 2}

\section{Approximate Bayesian Computation}

\subsection{Introdução}

Na inferência Bayesiana há uma necessidade recorrente de gerar observações de dados de interesse, e os motivos para tal necessidade são variados. Em grande parte das análises bayesianas essa necessidade é oriunda ao fato da distribuição de probabilidade a posteriori não possuir uma forma analítica fechada, o que é bastante frequente quando não é possível trabalhar dentro da famílias de distribuições conjugadas usuais, que são modelos mais simples e amplamente conhecidos.

Quando não se tem uma forma analítica fechada, a obtenção de resumos (como esperança, variância, máximo, mínimo, entre outros) a posteriori fica bastante dificultada. A geração de observações de uma distribuição de probabilidade é de grande ajuda neste caso, já que existem métodos numéricos que possibilitam calcular probabilidades, estimar densidades, fazer qualquer manipulação (inferência) que se deseje, e para isso basta ter um número de observações suficientemente grande.

Alguns dos algoritmos que geram dados observáveis (também chamados de amostradores) mais utilizados são baseados em $\mathbf{M C M C}$, sigla para Markov Chain Monte Carlo, um algoritmo que propõem gerar observações de uma deseja da distribuição de probabilidade através da evolução de cadeias de Markov. Pode-se dizer que o amostrador de Gibbs (Casella e George, 1992) é uma das variantes do $\boldsymbol{M C M C}$ mais utilizadas em análises devido a facilidade de implementá-lo. Outra variante basante utilizada é o algoritmo de Metropolis-Hasting (Gamerman e Lopes, 2006), mais robusto que o amostrador de Gibbs. Em esmagadora maioria os métodos de simulação necessitam, de alguma forma, da função de verossimilhança, geralmente através de manipulações algébricas.

O $\boldsymbol{A} \boldsymbol{B} \boldsymbol{C}$, também conhecido como likelihood-free method, é um amostrador que gera dados observáveis sem necessitar calcular, ou efetuar cálculos, em uma função de verossimilhança. Sua gênese, funcionamento e peculiaridades serão apresentado a seguir, bem como uma breve discussão sobre o que é verossimilhança, e se o $\boldsymbol{A} \boldsymbol{B} \boldsymbol{C}$ a utiliza ou não.

\subsection{Função de verossimilhança}

É usualmente dito que a função de verossimilhança nada mais é que a função de probabilidade (função densidade de probabilidade) vista em função do parâmetro, usualmente retratado por $\theta$, com uma observação amostral $x$, podendo ser uma única observação ou uma amostra com várias observações, mas fixa, já observada (DeGroot, 1989, capítulo 6). Esse fato faz com que a função do parâmetro não seja mais uma função de probabilidade, mas sim uma função, de qualquer, exceto de probabilidade ou densidade de probabilidade. Em Fisher (1922, pag. 310) é dito "The Likelihood that any parameter (or set of parameters) should have any assigned value (or set of values) is proportional that if this were so, the totality of observations should be that observed." Portanto a função pode ser escrita como:

$$
L(\theta) \propto f(x \mid \theta) .
$$

A verossimilhança de algum parâmetro $(\theta)$, dado a observação de uma amostra $(x)$ de tamanho 1(um), ou seja, uma única observação oriunda de uma população (ou variável) discreta, pode ser escrita da forma:

$$
L(\theta \mid x)=P(x \mid \theta) .
$$

Considerando agora uma amostra de tamanho $n, \mathbf{x}=\left(x_{1}, x_{2}, \ldots, x_{n}\right)$ (supondo observações inde- 
pendentes e identicamente distribuídas), pode-se escrever:

$$
L(\theta \mid \mathbf{x})=\prod_{i=1}^{n} P\left(x_{i} \mid \theta\right) .
$$

Analogamente, quando é observada uma amostra, de tamanho um e de tamanho $n$, respectivamente, de uma população (ou variável) continua, tem-se:

$$
\begin{aligned}
L(\theta \mid x) & =f(x \mid \theta) ; \\
L(\theta \mid \mathbf{x}) & =\prod_{i=1}^{n} f\left(x_{i} \mid \theta\right) .
\end{aligned}
$$

Em Bayarri e DeGroot (1992), além da definição supracitada, é dito que a função de verossimilhança, usualmente, também pode ser considerada como sendo proporcional a função de probabilidade, ou função de densidade de probabilidade, de quantidade observadas em um determinado experimento, condicionadas a quantidades não observadas neste mesmo experimento,

$$
L(\theta) \propto f(\text { o que foi observado|o que não foi observado) }
$$

De maneira mais simples, esta forma de pensar abre um precedente para interpretar a função de verossimilhança como sendo o condicionamento da amostra observada nas amostras que poderiam ser observadas, mas não foram. Assim, não há um interesse direto no parâmetro $\theta$, caso haja um no problema em estudo.

Note que a única diferença entre as duas interpretações é exatamente o que é considerado, ou não, parâmetro. Na primeira abordagem, equação 2.1, o parâmetro, geralmente, é considerado uma quantidade fixa, mas desconhecida. Já na segunda abordagem, equação 2.4 , isso pode não necessariamente acontecer. Esse fato fica mais claro com o exemplo contido em Bayarri e DeGroot (1992), exemplo 2.2.1.

Exemplo 2.2.1. Considere um problema em que, para cada valor do parâmetro $\theta$, uma variável aleatória $Y$ tem função densidade de probabilidade $f(y \mid \theta)$. Considera-se que $Y$ não pode ser, por si só, observada, mas sim pode-se observar uma pertubação aleatória $X$ de $Y$. Isto é, assume-se que o que é observado é uma realização da variável aleatória $X$ com função densidade de probabilidade condicional $f(x \mid y, \theta)=f(x \mid y)$.

Qual é a função de verossimilhança para esse problema? Existem diversas possibilidades. Primeiro deve-se decidir onde o valor desconhecido de y de $Y$ deve ser incluída na função de verossimilhança. Pode-se argumentar que, já que y não pode ser, nem nunca será, observado, é mais apropriado que não seja incluída ao se definir a função de verossimilhança, e assim definir:

$$
L_{0}(\theta) \propto f(x \mid \theta) .
$$

Por outro lado, já que a formulação básica do problema é em função de y, então pode-se pensar que y deve ser levada em consideração na função de verossimilhança (este caso seria óbvio se houvesse interesse também em y). Neste caso precisa-se decidir em como considerar y, como uma realização de uma variável aleatória, e portanto ficando a esquerda de |, da seguinte forma,

$$
L_{1}(\theta) \propto f(x, y \mid \theta),
$$

ou considerar y como uma quantidade não observada, e desconhecida, e assim fazendo-o ficar a direita de |, resultando uma função de verossimilhança da seguinte forma:

$$
L_{2}(\theta) \propto f(x \mid y, \theta)=f(x \mid y) .
$$

Qual função de verossimilhança usar não é, a principio, fácil de escolher, e é necessário realizar 
um julgamento de caráter subjetivo para poder decidir qual usar.

A motivação desse exemplo é mostrar que a função de verossimilhança pode ser ambígua e difícil de ser definida mesmo em problemas com um nível baixo de complexidade. Assim, a afirmação feita por Bayarri e DeGroot (1992) ("...there is not such a thing as ' $a$ ' likelihood function that can be unambigouosly defined in all statistical problems.") ganha bastante força. Não há uma definição única da função de verossimilhança para todos os problemas estatísticos. Com isso em mente um questionamento pode ser levantado: um conceito, tão difundido na estatística, pode ser delimitado somente pela definição de uma única função, que se mostrou ambígua? Pode-se considerar que o modelo probabilístico, do experimento de interesse, é um "sinônimo" da verossimilhança, no sentido de que ambos podem ser facilmente confundidos? O exemplo 2.2.1 deixa claro que função de verossimilhança e modelo probabilístico, função (densidade) de probabilidade, são distintas, apesar de uma depender da outra.

\subsection{Algoritmo $A B C$}

Em qualquer experimento sempre são feitas suposições sobre os dados observáveis. Essas suposições sempre são projetadas na função de verossimilhança, e algumas dessas suposições tornam a função de verossimilhança intratáveis, deixando qualquer cálculo impossível, se não computacionalmente bastante custoso. Nos casos em que a intratabilidade da função de verossimilhança não é tão extrema, algoritmos $\boldsymbol{M C M C}$ são bastante utilizados, mas o que pode ser feito quando a intratabilidade é tão grande que um algoritmo $M C M C$ demoraria um tempo maior do que o disponível para gerar uma amostra desejável? Nesta seção está apresentado uma alternativa para casos como esse, o algoritmo $\boldsymbol{A B C}$ (Approximate Bayesian Computation), que não utiliza a função de verossimilhança. Por esse motivo, esse método é chamado de likelihood-free.

\subsubsection{Gênese do Approximate Bayesian Computation}

A primeira aparição do $\boldsymbol{A B C}$, ainda como um conceito apareceu em Rubin (1984). nesse trabalho é dito que: "Suppose the model for data set $X$ is given by $f(X \mid \theta) p(\theta)$ where $\theta$ is the parameter whose posterior distribution is to be calculated. Of course, the posterior distribuition of $\theta$ given observed $X$ is calculated via Bayesian's theorem, but how is conceptual content of this theorem easily conveyed? Consider the simple description.

Suppose we first draw equally likely values of $\theta$ from $p(\theta)$, and label this $\theta_{1}, \ldots, \theta_{s}$. The $\theta_{j}, j=$ $1, \ldots, s$ can be thought of as representing the possible populations that might have generated the observed $X$. For each $\theta_{j}$, we now draw an $X$, that might have been observerd under the full model $f(X \mid \theta) p(\theta)$. Now some of the $X$ will look just like the observerd $X$ and many will not; of course, subject to the degree of rounding and the number of possible values of $X, s$ might have to be very large in order to find generated $X_{j}$ that agree with observerd $X$, but this creates no problem for our conceptual experiment. Suppose we collect together all $X_{j}$ match the observerd $X$, and then all $\theta_{j}$ that correspond to these $X_{j}$. This collection of $\theta_{j}$ represents the values of $\theta$ that could have generated the observerd $X$; formally, this collection of $\theta$ values represents the posterior distribution of $\theta$."

De maneira resumida, a ideia motriz do $A B C$ é a suposição de que, um valor $\theta$ oriundo da distribuição de $\theta \mid X=x$ é capaz de gerar, simular, um conjunto de dados igual, ou suficientemente próximos como mostrado a seguir, do conjunto de dados originalmente observado.

Essa ideia simples, desenvolvida por Rubin (1984), acabou se tornando uma alternativa computacional para o processo inferencial. Os casos que apresentam uma função de verossimilhança intratável, beneficiam-se bastante do algoritmo $\boldsymbol{A} \boldsymbol{B} \boldsymbol{C}$. Isso pode ser visto mais detalhadamente nos capítulos 4 e 5 .

A transcrição, para pseudo-código, da ideia presente em Rubin (1984) está presente no algoritmo 1. Este algortimo também pode ser encontrado em outros trabalhos, que contam a historia do $\boldsymbol{A} \boldsymbol{B} \boldsymbol{C}$, como por exemplo Marin et al. (2011) que, além disso apresentam outras versões do $\boldsymbol{A} \boldsymbol{B} \boldsymbol{C}$.

Para melhor entendimento do algoritmo 1, suponha que se deseja gerar amostras da distribuição a posteriori de uma quantidade não observável de um determinado experimento. Seja $\theta$ essa quantidade desconhecida e $\pi(\theta)$ sua distribuição de probabilidade a priori. Seja $X$ uma variável observável e $x$ o valor observado de $X$, podendo ser uma única ou vámrias observações. Suponha 
um modelo probabilístico para a variável $X$ dado o valor da quantidade desconhecida $\theta$. Denota-se por $f(x \mid \theta)$ tal modelo probabilístico. A verossimilhança gerada pela observação do experimento, já definida anteriormente, é $L(\theta \mid x)$. Assim a distribuição de probabilidade a posteriori de $\theta \mid X=x$ é dada por $\pi(\theta \mid X=x) \propto L(\theta \mid x) \pi(\theta)$.

Considerando um caso em que se tem uma função de verossimilhança intratável, mas em que se sabe como gerar uma observação de $f(\cdot \mid \theta)$, um exemplo disso está bem detalhada no capítulo 3 . O algoritmo 1, gera um $\theta$ da distribuição priori, denominado $\theta^{\prime}$ (passo 3). Então, com $\theta^{\prime}$ é gerado uma amostra $y$ oriunda de $f\left(\cdot \mid \theta^{\prime}\right)$ (passo 4). Se a amostra simulada é igual a amostra observada, então $\theta^{\prime}$ é uma observação de $\pi(\theta \mid X=x)$ (Passo 5).

Perceba que o $\boldsymbol{A} \boldsymbol{B} \boldsymbol{C}$ necessita, de maneira vital, que haja uma maneira de replicar o experimento observado: se não se sabe como fazer isso, então não há como implementar qualquer versão do $\boldsymbol{A} B \boldsymbol{C}$.

Note que em nenhum momento foi feita nenhuma manipulação, algébrica ou numérica, na função de verossimilhança. O processo inferencial, a maneira de como a informação de $\theta$ é obtida através da amostra observada, $x$, baseia-se somente no ato de replicar o experimento observado e anotar seus resultados. Por esse motivo o $\boldsymbol{A} \boldsymbol{B} \boldsymbol{C}$ também é chamado de likelihood-free method.

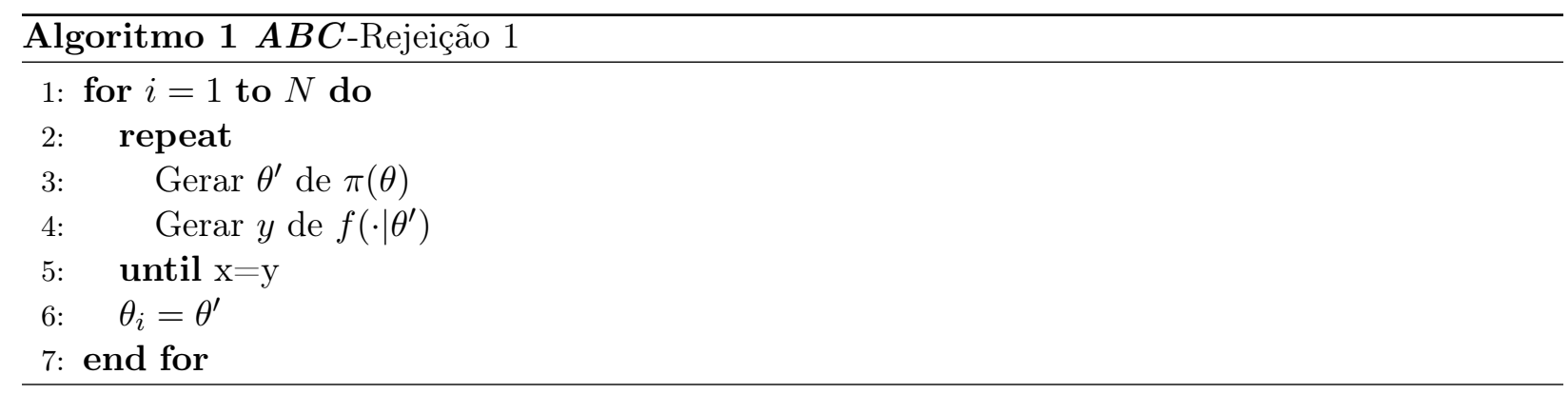

A prova matemática de que uma amostra gerada pelo $\boldsymbol{A B C}$ pode ser considerada como uma amostra da distribuição a posteriori de interesse é dada a seguir. Seja $f\left(\theta_{i}\right)$ a densidade de $\theta_{1}$ gerada pelo algoritmo 1. Assim,

$$
\begin{aligned}
f\left(\theta_{i}\right) & \propto \sum_{y \in \mathcal{X}} \pi\left(\theta_{i}\right) f\left(y \mid \theta_{i}\right) \mathbf{I}_{x}(y)=\pi\left(\theta_{i}\right) f\left(x \mid \theta_{i}\right) \\
& \propto \pi\left(\theta_{i} \mid x\right),
\end{aligned}
$$

onde $\mathcal{X}$ é o conjunto, enumerável, de valores possíveis de $x$.

Uma demonstração deveras simples, mas o suficiente para dar credibilidade matemática ao $A B C$.

\subsubsection{Observando variáveis aleatórias contínuas}

Do ponto de vista computacional, a condição de parada do algoritmo 1, passo 5, é inalcançável, quando os valores $x$ e $y$ pertencem ao conjunto dos números reais.

Sofrendo desse problema, observando valores contínuos, Pritchard et al. (1999) utilizam o $\boldsymbol{A} \boldsymbol{B} \boldsymbol{C}$ de forma modificada. Com o intuito de relaxar a condição de igualdade do algoritmo 1. Isso foi possível utilizando mecanismos,que hoje são chamados de tunning parameters.

Devido ao fato de observar uma amostra de tamanho grande, Pritchard et al. sumarizam a amostra, condensando asim a informação contida nela e assume que a condição de igualdade entre as amostras, geradas e observadas, pode ser substituída por: a amostra gerada, devidamente sumarizada, está suficientemente perto da amostra observada, também devidamente sumarizada.

Com esse relaxamento, a ideia original de Rubin (1984) se modifica. Agora, se um valor $\theta^{\prime}$ gerado de $\pi(\theta)$ pode gerar uma amostra $y$ na vizinhança da amostra observada $x$, então esse valor $\theta^{\prime}$ específico é considerado como uma realização da distribuição a posteriori $\pi(\theta \mid x)$.

A transcrição, para pseudo-código, do algoritmo apresentado em Pritchard et al. (1999) encontrase logo a baixo, algoritmo 2. Nele são presentes os seguintes tunning parameters: 
$\eta(\cdot)$ é a função sumário, que é utilizada para resumir a informação da amostra em uma ordem de grandeza menor que a da amostra(e.g. estatísticas suficientes);

$\rho(\cdot)>0$ é a função distância, que vai informar quão longe, ou perto, as duas amostras, observada e a simulada, estão;

$\epsilon>0$ é a distância máxima tolerada entre as duas amostras.

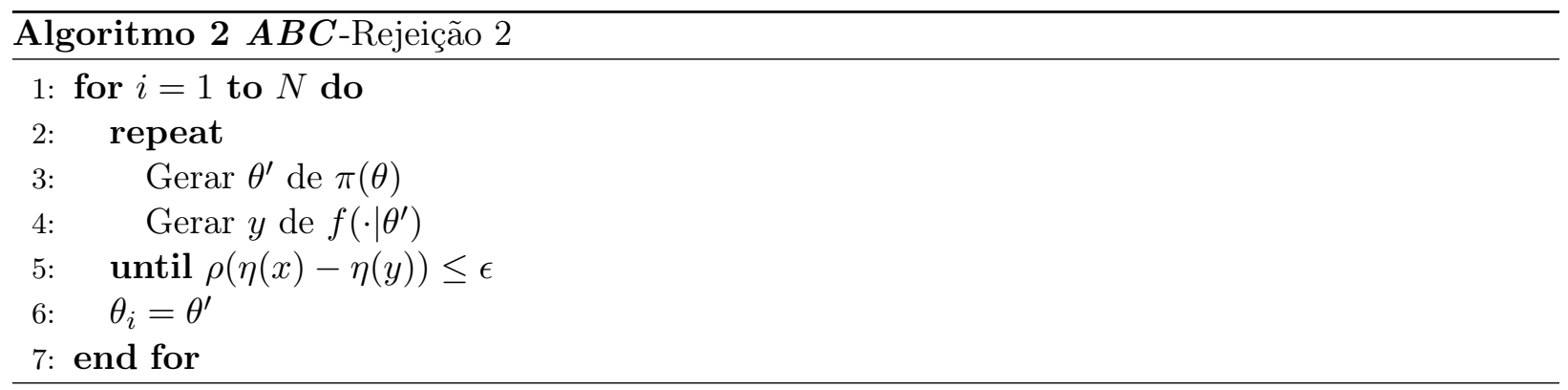

A demonstração teórica de que a amostra gerada pelo algoritmo 2 segue, aproximadamente, a distribuição de probabilidade a posteriori de interesse segue abaixo.

Considere o conjunto $A_{\epsilon, x}=\{y \in \mathcal{X} \mid \rho(\eta(x)-\eta(y)) \leq \epsilon\}$, todos os valores $y$ que satisfizeram a condição de aceitação do algoritmo 2 , para um $\epsilon$ determinado. Note que no contexto que envolve o algoritmo 2, quando observamos variáveis aleatórias continuas, $\mathcal{X}$ não é enumerável.

A distribuição a posteriori conjunta de $\left(\theta_{i}, y\right)$ é da forma:

$$
\pi_{\epsilon}(\theta, y \mid x)=\frac{\pi(\theta) f(y \mid \theta) \mathbf{I}_{A_{\epsilon, x}}(y)}{\int_{A_{\epsilon, x} \times \Theta} \pi(\theta) f(y \mid \theta) d \theta} .
$$

Assim,

$$
\pi_{\epsilon}(\theta \mid x)=\int \pi_{\epsilon}(\theta, y \mid x) d y \approx \pi(\theta \mid x)
$$

A qualidade da aproximação, que pode não estar muito clara na demonstração acima, depende da escolha conjunta de $\eta$ e $\epsilon$. A escolha de um sumário de amostra $\eta$ representativa o suficiente aliado com uma tolerância $\epsilon$ pequena o suficiente, deve produzir uma aproximação, suficientemente boa.

Quando $\epsilon$ é igual a zero, mesmo caso do $\boldsymbol{A B C}$-Rejeição quando se observam variáveis discretas, tem-se a amostra exata da distribuição alvo, mas, quando são observadas variáveis contínuas, escolher um $\epsilon$ igual a zero revela-se uma impossibilidade computacional. A escolha do valor de $\epsilon$ é uma negociação entre os custos computacionais e a qualidade da aproximação. Uma discussão mais aprofundada sobre as diferenças entre $\pi_{\epsilon}(\theta \mid x)$ e $\pi(\theta \mid x)$, através de resultados empíricos, pode ser encontrada em Sisson et al. (2007), e resultados teóricos sobre limites superiores, em casos bem específicos, para $\epsilon$ podem ser vistos em Dean et al. (2014).

Quando se tem um conjunto de dados com várias dimensões, sumários de amostra de dimensões menores, condensando a maior quantidade de informação possível da maneira mais simples que se possa, são essenciais. Mas nem sempre é possível condensar a informação dos dados da maneira que é mais útil. A má escolha de funções sumários, além de aumentar a diferença entre $\pi_{\epsilon}(\theta \mid x)$ e $\pi(\theta \mid x)$, podem levar a uma perda de informação o que implica numa inferência de má qualidade (Csilléry et al., 2010). Uma revisão pode métodos para escolher $\eta$ pode ser encontrada em Blum et al. (2013).

É importante frisar que em cada caso, a escolha de $\eta$ e $\epsilon$ deve ser feita depois de investigações da qualidade da amostra gerada, em contra partida com custos computacionais envolvidos na execução do algoritmo. 


\subsection{3 $M C M C-A B C$}

Outras variações do algoritmo $\boldsymbol{A B C}$, desenvolvidas para superar dificuldades encontradas em diversos problemas, podem ser encontradas em Marin et al. (2011). Uma dessas variações é o

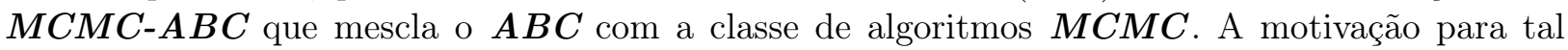
junção foi a tendência em gerar pontos com baixa probabilidade a posteriori que o $A B C$-Rejeição tem em alguns casos. O $\boldsymbol{M} \boldsymbol{C} \boldsymbol{M} \boldsymbol{C}-\boldsymbol{A} \boldsymbol{B} \boldsymbol{C}$ fez sua primeira aparição em Marjoram et al. (2003), com a ideia de adicionar uma nova condição de aceitação para $\theta^{\prime}$, condição essa também presente no algoritmo de Metropolis-Hastings, além da adição de um kernel de transição para gerar novos $\theta^{\prime} s$ dependendo do ultimo $\theta^{\prime}$ aceito. A transcrição do $\boldsymbol{M C M C}-\boldsymbol{A} \boldsymbol{B C}$ pode ser vista a seguir, algoritmo 3 .

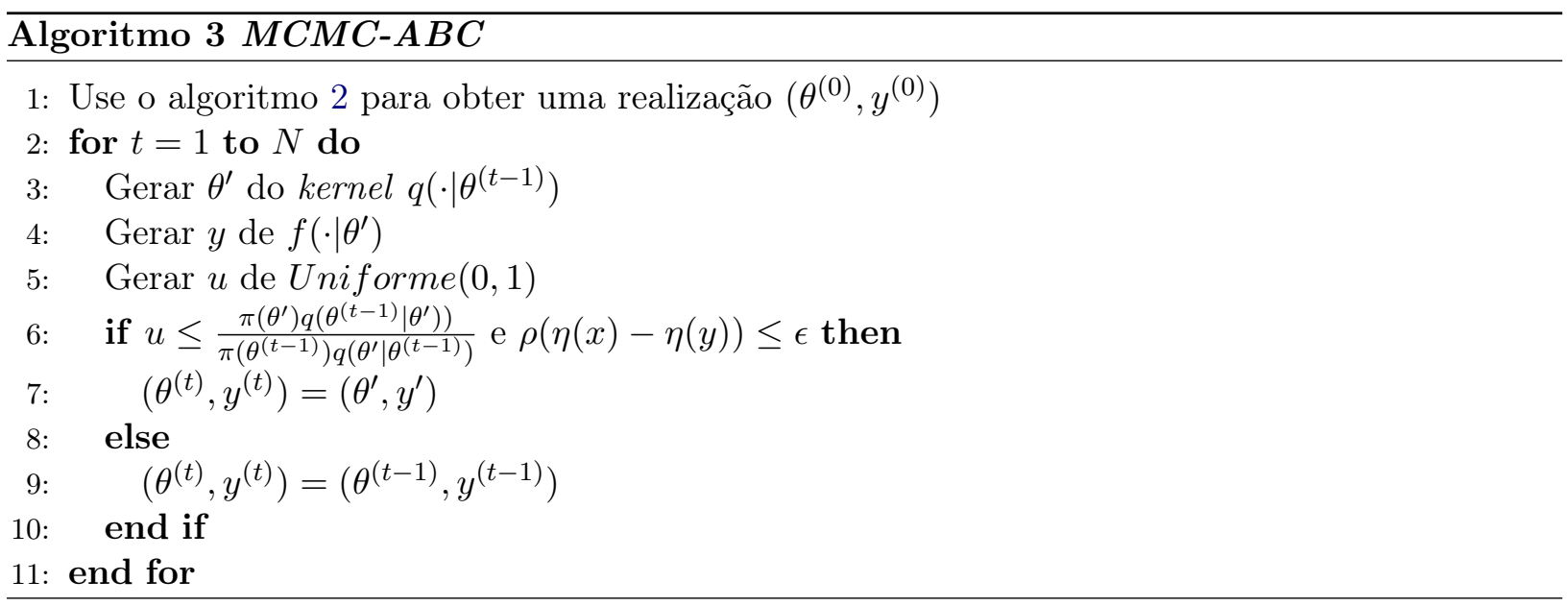

Ao custo de aumentar a complexidade computacional do $A B C$-Rejeição, que pode ser impraticável em algumas situações, o $\boldsymbol{M C M C}-\boldsymbol{A} \boldsymbol{B C}$ promete diminuir, talvez até eliminar, a tendência do $\boldsymbol{A} \boldsymbol{B} \boldsymbol{C}$-Rejeição gerar pontos em regiões de baixa probabilidade a posteriori.

\subsubsection{Noisy $A B C$}

Outra variação do $\boldsymbol{A} \boldsymbol{B} \boldsymbol{C}$, também comentada em Marin et al. (2011), mas apresentada pela primeira vez em Wilkinson (2008) chama-se Noisy $\boldsymbol{A} \boldsymbol{B} \boldsymbol{C}$. A particularidade desse método é aceitar que o $\boldsymbol{A} \boldsymbol{B} \boldsymbol{C}$ gera amostras perfeitas, mas da distribuição errada.

Semelhante a um modelo linear, ou modelo linear generalizado, onde os dados são observações de um modelo adicionado de um erro aleatório, Wilkinson (2008) interpreta a distribuição gerada pelo $\boldsymbol{A} \boldsymbol{B} \boldsymbol{C}$ como se fosse o melhor modelo de predição possível e assume que há uma discrepância entre esse melhor modelo de predição e os dados observados. Essa discrepância pode representar tanto um erro de medida nos dados como um erro de modelo. Fazendo esta discrepância ser como o erro aleatório de um modelo linear, é possível escrever os dados observados da seguinte forma:

$$
x=P(\cdot \mid \hat{\theta})+\delta,
$$

uma realização do modelo com o melhor argumento $\hat{\theta}$, adicionado de um termo aleatório $\delta$. Por ser um termo aleatório é assumido uma distribuição para $\delta$, denominada por $\pi_{\delta}(\cdot)$.

A condição de aceitação do $\boldsymbol{A} \boldsymbol{B} \boldsymbol{C}$, na metodologia presente em Wilkinson (2008), baseia-se na distribuição $\pi_{\delta}(\cdot)$ avaliada no ponto $x-y$, o que torna a regra de aceitação variável a cada iteração do algoritmo. Detalhes em como construir $\pi_{\delta}(\cdot)$ encontram-se em Wilkinson (2008).

Todos as simulações desse capítulo, bem como de todo o trabalho, feitas usando o $\boldsymbol{A} \boldsymbol{B C}$ foram geradas pelo $\boldsymbol{A} \boldsymbol{B} \boldsymbol{C}$-Rejeição. Nenhuma outra variação foi utilizada, pois tendo em vista a alta complexidade ds modelos discutidos neste trabalho, manter a simplicidade computacional do $\boldsymbol{A} \boldsymbol{B C}$ tornou-se prioridade. 


\subsection{Toy model}

Para ilustrar o funcionamento do $\boldsymbol{A B C}$, ele foi implementado para gerar amostras de duas distribuições a posteriori de modelos Bayesianos já conhecidos, toy models. Em um dos modelos a variável observada é discreta e no outro é uma variável contínua. Isso permite observar os algoritmos 1 e 2 em ação. Os modelos em estudo são:

1. Considerando $\mathbf{X}$ uma sequência de $n$ ensaios de Bernoullis

$$
\begin{aligned}
\theta & \sim \operatorname{Beta}(a, b) \\
\mathbf{X} \mid \theta & \sim \operatorname{Bernoulli}(\theta), \\
\theta \mid \mathbf{X}=\mathbf{x} & \sim \operatorname{Beta}\left(a+\sum_{i=1}^{n} x_{i}, b+1-\sum_{i=1}^{n} x_{i}\right)
\end{aligned}
$$

2. Considerando uma amostra de tamanho $n$

$$
\begin{aligned}
\tau & \sim \operatorname{Gama}\left(\alpha_{0}, \beta_{0}\right), \\
\mu \mid \tau & \sim \operatorname{Normal}\left(\mu_{0},(\lambda \tau)^{-1}\right), \\
X \mid \mu, \tau & \sim \operatorname{Normal}\left(\mu, \tau^{-1}\right), \\
\tau \mid X=x & \sim \operatorname{Gama}\left(\alpha_{n}, \beta_{n}\right), \\
\mu \mid \tau, X=x & \sim \operatorname{Normal}\left(\mu_{n},[(n+\lambda) \tau]^{-1}\right) ;
\end{aligned}
$$

onde

$$
\begin{aligned}
& \alpha_{n}=\alpha_{0}+n / 2, \\
& \beta_{n}=\beta_{0}+\frac{\sum_{i=1}^{n}\left(x_{i}-\bar{x}\right)^{2}}{2}+\frac{n \lambda\left(\bar{x}-\mu_{0}\right)^{2}}{2(n+\lambda)}, \\
& \mu_{n}=\frac{n \bar{x}+\lambda \mu_{0}}{n+\lambda} .
\end{aligned}
$$

Como é sabido, em outros métodos computacionais que, também utilizam parâmetros de tunning, Random Walk Chain $\boldsymbol{M C M C}$ por exemplo (Gamerman e Lopes, 2006, pag. 198), não existe uma regra geral para determinar o melhor valor para o parâmetro de tunning. Por isso foram escolhidos alguns valores para esses parâmetros, gerando assim alguns cenários diferentes em que o $\boldsymbol{A B C}$ será implementado.

$\mathrm{Na}$ escolha de $\eta(\cdot)$, tem-se a crença que usar um vetor cujas componentes são estatísticas suficientes, ou utilizar um vetor cujas as componentes são funções da amostra que estão parte de estatísticas suficientes, como o boxplot (um vetor cujas componentes são os três quartis), que é parte de uma estatística suficiente(estatísticas de ordem), devem gera amostras de boa qualidade. Dependendo das características amostrais (tendência central, dispersão, assimetria, curtose, etc.) de $\mathbf{x}$ que deseja-se replicar na amostra gerada $y$ será necessário uma função $\eta(\cdot)$ especifica.

Para escolher a função distância, $\rho(\cdot)$, a distância euclideana parece natural para isso, mas também foram testadas outras composições de distâncias.

O nível de tolerância $\epsilon$ funciona como em qualquer outro algoritmo: quanto menor, mais próximo estão as duas amostras, e mais tempo computacional é gasto para gerar uma amostra $y$ que satisfaça a condição de aceitação desejada.

\subsubsection{Beta-binomial}

O primeiro dos toy models a ser estudado é o modelo beta-binomial. O $\boldsymbol{A} \boldsymbol{B C}$ configurado para este caso está apresentado em seguida, algoritmo 4.

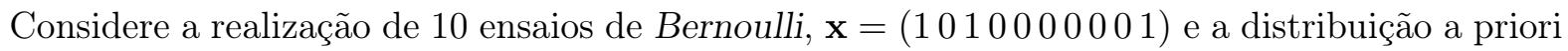
$\theta \sim \operatorname{beta}(1 / 2,1 / 2)$. Pela equação 2.9 tem-se que,

$$
\theta \mid X=\mathbf{x} \sim \operatorname{Beta}(7 / 2,15 / 2) .
$$




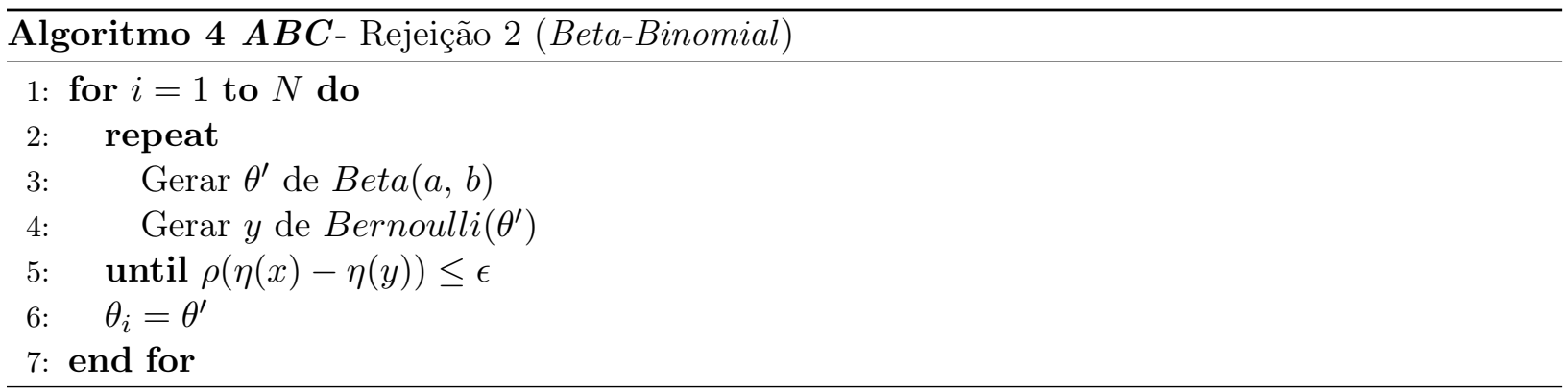

O algoritmo $\boldsymbol{A} \boldsymbol{B} \boldsymbol{C}$-Rejeição 2 (Beta-Binomial) foi configurado em quatro cenários diferentes

1. $\eta(x)=\bar{x}, \rho(\eta(x)-\eta(y))=(\bar{x}-\bar{y})^{2}$ e $\epsilon=10^{-2}$;

2. $\eta(x)=\mathbf{x}, \rho(\eta(x)-\eta(y))$ é a distância euclidiana e $\epsilon=10^{-2}$

3. $\eta(x)=\bar{x}, \rho(\eta(x)-\eta(y))=(\bar{x}-\bar{y})^{2}$ e $\epsilon=10^{-6}$;

4. $\eta(x)$ é um vetor cujas componentes são os quantis de ordem $25 \%, 50 \%, 75 \%, \rho(\eta(x)-\eta(y))$ é a distância euclidiana $\epsilon=10^{-6}$.

Note que, quando o algoritmo 2 é implementado com $\eta(\cdot)$ descrito como no segundo cenário, independente das escolhas de $\rho(\cdot)$ e de $\epsilon$, o algoritmo executado acaba sendo o algoritmo 1.

Para cada cenário foram gerados 100 amostras de tamanho 1000 cada uma, e com essas amostras obtiveram-se as seguintes informações:

1. Para gerar uma amostra de tamanho 1000 foi necessário gerar, em média, $7153 \theta^{\prime}$, em outras palavras é necessário gerar $8 \theta^{\prime}$ para aceitar 1 ;

2. Para gerar uma amostra de tamanho 1000 foi necessário gerar, em média, $1840000 \theta^{\prime}$, em outras palavras é necessário gerar $1841 \theta^{\prime}$ para aceitar 1;

3. Para gerar uma amostra de tamanho 1000 foi necessário gerar, em média, $153200 \theta^{\prime}$, em outras palavras é necessário gerar $154 \theta^{\prime}$ para aceitar 1 ;

4. Para gerar uma amostra de tamanho 1000 foi necessário gerar, em média, $153300 \theta^{\prime}$, em outras palavras é necessário gerar $154 \theta^{\prime}$ para aceitar 1 .

Ainda utilizando as réplicas, os gráficos da figura 2.1 foram feitos com a intenção de saber quão aproximado está a amostra da posteriori gerada pelo $\boldsymbol{A B C}$ da densidade da distribuição a posteriori desejada (equação 2.12).

Em cada cenário, cada uma das réplicas foi ordenada, gerando assim as estatísticas de ordem. Calculou-se a média de cada uma das estatística de ordem nas 100 réplicas, gerando assim uma amostra composta pelas médias de cada estatística de ordem. Esta amostra composta pelas médias das estatísticas de ordem será denominada amostra média. Na figura 2.1, estão representados cada uma das amostras médias para cada cenário.

Percebe-se que a configuração do cenário 1 não é tão boa quanto as dos outros três cenários, pois foi a que mais se afastou da distribuição alvo, equação 2.9 , embora tenha sido a que necessitou de menos interações para a amostra. A amostra gerada com as configurações do cenário 2 , teoricamente, tem a melhor qualidade, pelo fato de usar toda a amostra $\mathbf{x}$ como função sumário. Mas foi o caso em que o $\boldsymbol{A} \boldsymbol{B} \boldsymbol{C}$ mais foi dispendioso computacionalmente, já que necessitou mais de 10 vezes mais interações que o $\boldsymbol{A} \boldsymbol{B} \boldsymbol{C}$ nos outros dois cenários, para gerar a amostra. As configurações dos cenários 3 e 4 geraram amostras muito próximas da densidade alvo, tanto quanto as do cenário 2, e com uma taxa de aceitação bem maior, mostrando-se as melhores alternativas, dentre os quatro cenários, aliando qualidade da amostra com consumo de recursos computacionais intermediário. Como comentado anteriormente, a escolha de $\epsilon$ é uma negociação entre recursos computacionais e qualidade da amostra. 


\subsubsection{Normal-gama}

O segundo toy model estudado neste capítulo é um modelo conjugado bastante conhecido, modelo normal-gama. $\mathrm{O}$ algoritmo 2 foi configurado para gerar amostras da distribuição de $(\mu, \tau) \mid X=$ $x$ apresentado em seguida.

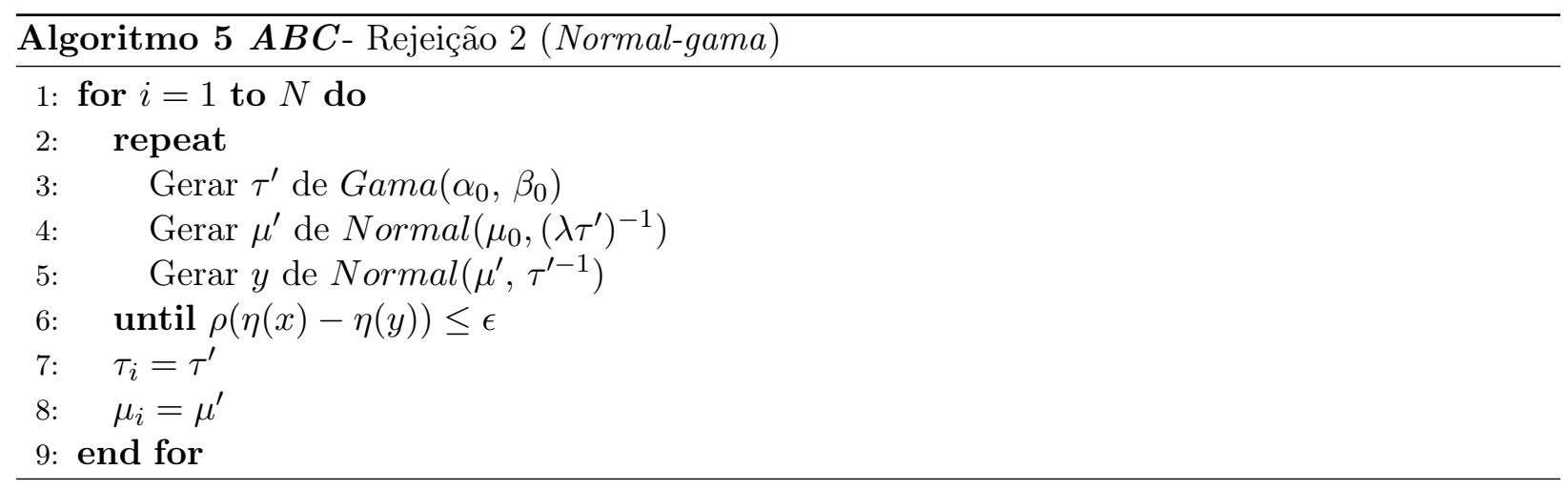

Os hiperparâmetros foram escolhidos da seguinte forma, $\lambda=1, \mu_{0}=0.45, \alpha_{0}=1.53$ e $\beta_{0}=0.81$. Novamente foram escolhidos cenários diferentes para estudar o desempenho do $\boldsymbol{A} \boldsymbol{B} \boldsymbol{C}$. Estes estão apresentados a seguir:

1. $\eta(x)=\left(\sum_{i=1}^{n} x_{i}, \sum_{i=1}^{n} x_{i}^{2}\right), \rho(\eta(x)-\eta(y))$ é a distância euclidiana e $\epsilon=10^{-2}$;

2. $\eta(x)$ é um vetor cujas as componentes são os quantis de ordem $25 \%, 50 \%, 75 \%, \rho(\eta(x)-\eta(y))$ é a distancia euclidiana e $\epsilon=10^{-2}$.

Novamente, para cada cenário, foram gerados 100 amostras de tamanho 1000, com uma mesma amostra observada para cada um dos cenários. A amostra observada nesse exemplo, x, tem tamanho 100 e possui $\left(\sum_{i=1}^{n} x_{i}, \sum_{i=1}^{n} x_{i}^{2}\right)=(40.46047,45.42041)$ e Quantis $(25 \%, 50 \%, 75 \%)=(-0.01946887,0.46127636,0.8$ A distribuição a posteriori de $(\mu, \tau) \mid \mathbf{x}$ pode ser escrita da seguinte forma:

$$
\begin{aligned}
\tau \mid \mathbf{X}=\mathbf{x} & \sim \operatorname{Gama}(51.53,15.33598) \\
\mu \mid \tau, \mathbf{X}=\mathbf{x} & \sim \operatorname{Normal}\left(0.4050541,(101 * \tau)^{-1}\right)
\end{aligned}
$$

Note que $\eta(x)$ é um vetor em qualquer um dos dois cenários.

Antes de apresentar os resultados das simulações é interessante ressaltar que no modelo betabinomial possui um parâmetro é escalar. Já o modelo normal-gama tem um vetor de parâmetros $(\mu, \tau)$. Não há como usar estatísticas de ordem, como foram usadas no toy model com o modelo Beta-Binomial, para os vetores observados. A alternativa utilizada foi calcular a função empírica de probabilidade acumulada para cada uma das réplicas geradas, e então calcular a média das funções empíricas ponto a ponto. Assim há a possibilidade de comparar a função empírica média de cada cenário com função de probabilidade acumulada da distribuição alvo.

Os seguintes resultados foram obtidos, para cada cenário:

1. Para gerar uma amostra da distribuição a posteriori de tamanho 1000 foi necessário gerar, em média, $14915067 \theta^{\prime}$. Significa que é necessário gerar $14916 \theta^{\prime}$ para aceitar 1 desses 14916;

2. Para gerar uma amostra da distribuição a posteriori de tamanho 1000 foi necessário gerar, em média, 298800.1 $\theta^{\prime}$. Significa que é necessário gerar $299 \theta^{\prime}$ para aceitar 1 desses 299.

Na figura 2.2 são apresentados as curvas de níveis das funções de probabilidade acumulada da distribuição alvo, figura (a), e das funções empíricas média de cada um dos cenários, figuras (b), (c). Percebe-se que dos dois cenários o que mais se aproxima da distribuição alvo é o segundo. 
Este cenário não trabalha com uma estatística suficiente como função sumário, mas sim uma parte dela. Como falado anteriormente, a suficiência, utilizada como sumário, ou parte da suficiência, aliada com a escolha da função de distância, deve gerar boas amostras. É imprescindível investigar cada caso a fim de determinar a melhor combinação possível. Quando as estatísticas suficientes foram usadas como função sumário, primeiro cenário, amostras y suficientemente próximas das amostras $\mathbf{x}$ (passo 6 do algoritmo 5) são geradas em grande quantidade, o que diminui a taxa de rejeição de $\theta^{\prime}$. Tal fato não acontece no segundo cenário, que utiliza uma outra estatística, não suficiência e, dificulta, restringe, a geração de amostras $\mathbf{y}$ próximas de $\mathbf{x}$ aumentando assim a taxa de rejeição de $\theta^{\prime}$. Esse fato pode explicar a razão da grande geração de pontos em uma região de baixa probabilidade a posteriori.

De modo geral, as suspeitas sobre a influência dos parâmetros de tunning foram comprovadas.A utilização de estatísticas suficientes, ou parte delas, como resumo das amostras, associada com a distância euclidiana fazendo o papel de $\rho(\cdot)$, tende a uma amostra mais plausível com a distribuição alvo. A tolerância $\epsilon$ influencia a velocidade computacional e a qualidade da amostra como prevíamos.

O fato do $\boldsymbol{A} \boldsymbol{B} \boldsymbol{C}$ gerar pontos amostrais regiões de baixa probabilidade a posteriori é comentado em Marin et al. (2011). O $\boldsymbol{M} \boldsymbol{C} \boldsymbol{M} \boldsymbol{C}-\boldsymbol{A} \boldsymbol{B} \boldsymbol{C}$, já exposto anteriormente foi criado com o intuito de resolver esse problema.

Como os modelos estatísticos apresentados nos capítulos seguintes tem uma complexidade computacional elevada, decidiu-se implementar somente o $\boldsymbol{A B C}$-Rejeição para não elevar inda mais a complexidade dos cálculos. Por esse motivo, os desempenho do $\boldsymbol{M C M C} \boldsymbol{C} \boldsymbol{A} \boldsymbol{C}$ não foi apresentado aqui. mas os dados sobre ele estão disponíveis em Campos e Wechsler (2012).

\subsection{Comparando o $A B C$ com um algoritmo $M C M C$}

Apenas com as informações apresentadas na seção anterior, não se pode afirmar se o $\boldsymbol{A} \boldsymbol{B} \boldsymbol{C}$ é um bom amostrador, se gera amostras confiáveis e se é de rápida execução computacional. Por isso é necessário compará-lo com a classe de algoritmos $M C M C$.

Foi decidido comparar as amostras geradas, pelo $\boldsymbol{A B C}$, para o segundo toy-model, modelo normal-gama, com as amostras geradas por um algoritmo $\boldsymbol{M C M C}$. O escolhido para este modelo foi o amostrador de Gibbs (veja Casella e George, 1992).

Como mostrado anteriormente o modelo normal-gama apresenta as seguintes distribuições a posteriori,

$$
\begin{aligned}
\tau \mid X=x & \sim \operatorname{Gama}\left(\alpha_{n}, \beta_{n}\right), \\
\mu \mid \tau, X=x & \sim \operatorname{Normal}\left(\mu_{n},[(n+\lambda) \tau]^{-1}\right) ;
\end{aligned}
$$

deste modo,

$$
\pi(\mu, \tau \mid X=x)=\frac{\sqrt{(n+\lambda) \tau}}{2 \pi} e^{-\frac{1}{2}\left(\mu-\mu_{n}\right)^{2}(n+\lambda) \tau} \frac{\beta_{n}}{\Gamma\left(\alpha_{n}\right)} \tau^{\alpha_{n}-1} e^{-\beta_{n} \tau} .
$$

Não é difícil ver que as distribuições condicionais completas, $\pi(\mu \mid \tau, X=x)$ e $\pi(\tau \mid \mu, X=x)$, que são necessárias para implementar o amostrador de Gibbs, coincidem com as distribuições a posteriori de $\tau \mid X=x$ e $\mu \mid \tau, X=x$. Utilizando a mesma amostra observada que foi utilizada na seção 2.4.2, pode-se obter osseguintes valores $\alpha_{n}=51.53, \beta_{n}=15.33598$ e $\mu_{n}=0.4050541$. O processo para obter uma amostra da distribuição de $\mu, \tau \mid X=x$ usando o amostrador de Gibbs encontra-se descrito abaixo, algoritmo 6.

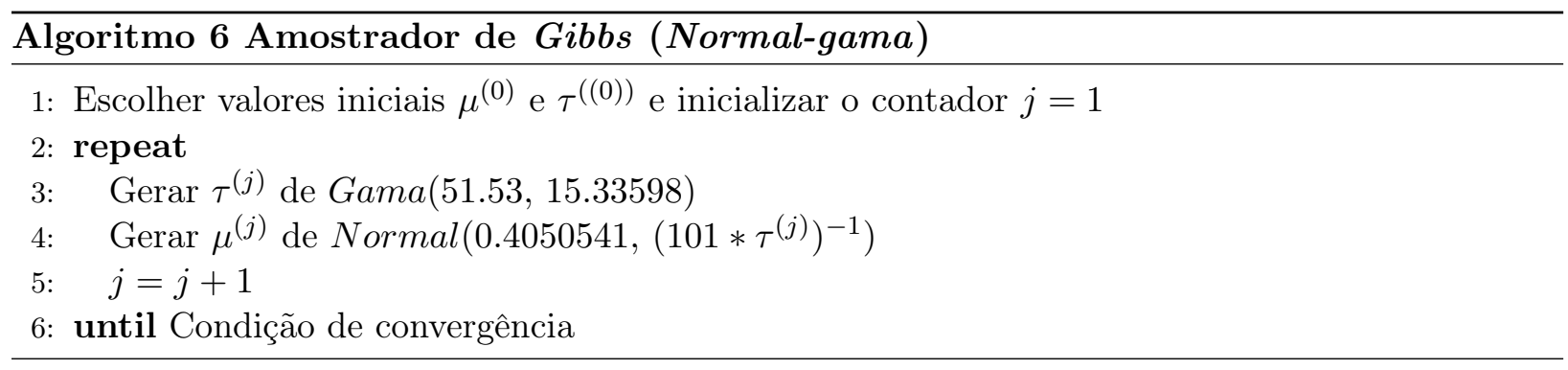


Foi escolhido utilizar um critério gráfico para diagnosticar a convergência da cadeia, monitorando a média ergódica das duas cadeias, uma para $\mu$ e outra para $\tau$. Foi estipulado que se a média ergódica na iteração $j$ for igual a média ergódica da interação $j-1$, com pelo menos 4 casas decimais de igualdade, e se essa igualdade se mantiver h por pelo menos 500 iterações, as cadeias terão atingido a convergência.

Por um motivo de comparação, decidiu-se iniciar sempre um novo par de cadeias de Markov para cada nova observação da amostra gerada. Deste modo é possível comparar o número de interações que o $\boldsymbol{A B C}$ necessitou para gerar uma observação com o número de iterações que o amostrador de Gibbs necessitou para gerar uma observação, descontando as 500 iterações obrigatórias para a constatação da convergência, além de não haver preocupação com a dependência entre as observações.

Tabela 2.1: Sumário do número de interações necessárias para gerar uma observação usando o amostrador de Gibbs

\begin{tabular}{ccccccc}
\hline \hline Mínimo & $1^{\circ}$ Quartil & Mediana & Média & $3^{\circ}$ Quartil & Máximo & Variância \\
\hline 858 & 1249 & 1367 & 1386 & 1504 & 2334 & 34778,59 \\
\hline \hline
\end{tabular}

Ao comparar a informação apresentada na tabela 2.1 com as informações contidas na seção 2.4.2, nota-se que, desconsiderando as 500 interações obrigatórias para a constatação de convergência, o amostrador de Gibbs necessitou, em média, de 1386 interações para gerar uma observação da amostra da distribuição de interesse, enquanto o $\boldsymbol{A} \boldsymbol{B} \boldsymbol{C}$, no melhor dos casos necessitou de apenas 299 interações, em média, para gerar uma observação.

Comparando os dois algoritmos apenas por esse critério, tem-se a impressão de que o $\boldsymbol{A} \boldsymbol{B C}$ é um algoritmo melhor, mas essa decisão pode estar equivocada. Também é necessário comparar a qualidade da amostra gerada por cada um dos amostradores. As amostras geradas pelo $\boldsymbol{A} \boldsymbol{B} \boldsymbol{C}$, primeiro e terceiro cenário, e Gibbs foram comparadas, nas figuras 2.4 e 2.5. Estes cenários, apresentados na seção 2.4.2, foram escolhidos por terem gerado as amostras que mais se aproximaram com a distribuição alvo (ver figura 2.2).

Com o auxílio das figuras 2.4 e 2.5 pode-se dizer que, para o modelo normal-gama, o amostrador de Gibbs conseguiu gerar melhores amostras, embora necessitasse de um pouco mais de 6 vezes mais interações que o $\boldsymbol{A} \boldsymbol{B} \boldsymbol{C}$ no cenário que gerou a melhor amostra, mas ainda assim o desempenho computacional do amostrador de Gibbs é considerado bom. Os gráficos na figura 2.4 confirmam o que já foi dito, o algoritmo $\boldsymbol{A} \boldsymbol{B} \boldsymbol{C}$-rejeição tende a gerar pontos em baixa área de probabilidade a posteriori. O amostrador de Gibbs conseguiu gerar de maneira satisfatória a cauda da distribuição alvo.

Note que, mesmo com as escalas dos gráficos na figura 2.5 sendo diferentes, é possível ver que as amostras gerada pelo $\boldsymbol{A} \boldsymbol{B} \boldsymbol{C}$ mais dispersas que a amostra gerada pelo amostrador de Gibbs, uma explicação para isso pode ser a tendência do $\boldsymbol{A} \boldsymbol{B} \boldsymbol{C}$-Rejeição de gerar pontos em regiões de baixa probabilidade a posteriori.

Se fosse necessário escolher um algoritmo como o melhor seria o amostrador de Gibbs pois mostrou-se mais confiável quando foi utilizado. Deve-se lembrar que este modelo em especifico é simples, e já era esperada essa "derrota" do $\boldsymbol{A} \boldsymbol{B} \boldsymbol{C}$. Mas para casos com uma função de verossimilhança de forma analítica intratável, o $A B C$ se mostrará um algoritmo mais eficiente do que visto até aqui, para gerar observações de modelos em estudo, como será mostrado nos próximos capítulos. 


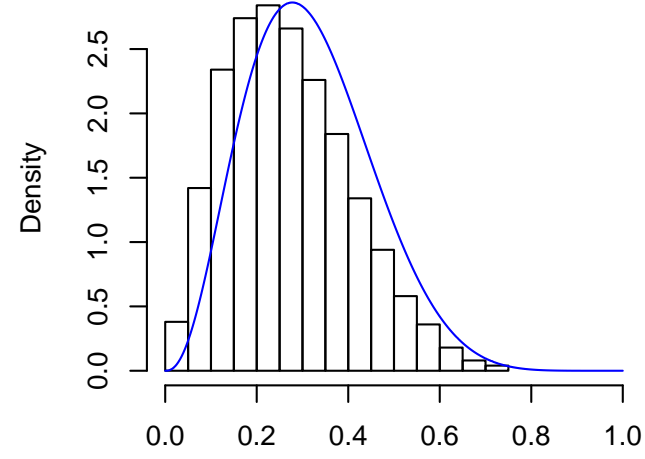

$\theta$

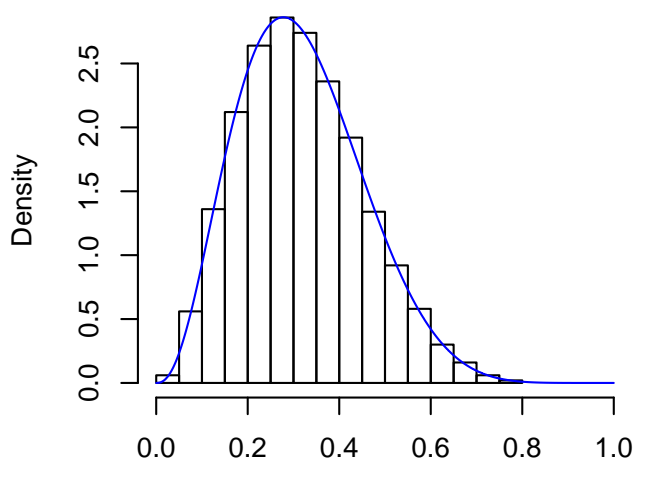

$\theta$

(c)

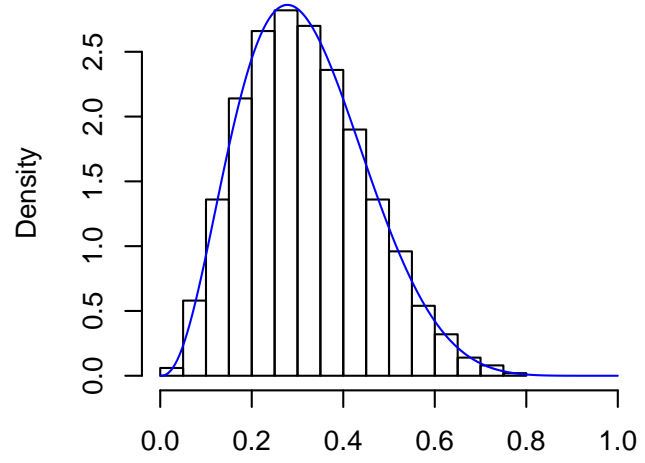

$\theta$

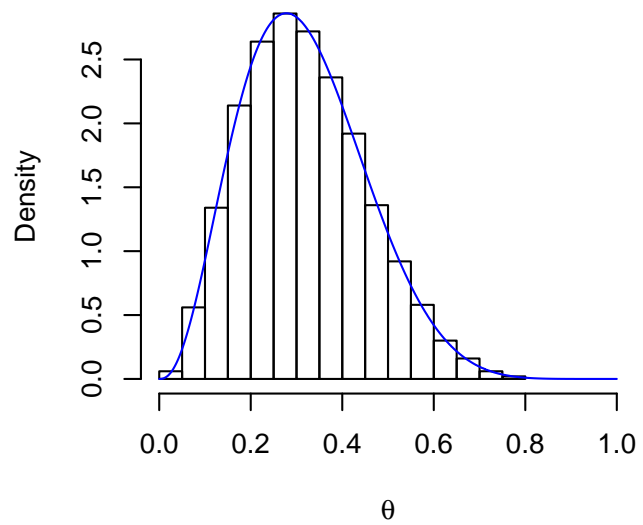

(d)

Figura 2.1: (a) Histograma da amostra média gerada no primeiro cenário comparada com a densidade a posteriori (equação 2.12); (b) Histograma da amostra média gerada no segundo cenário comparada com a densidade a posteriori (equação 2.12); (c) Histograma da amostra média gerada no terceiro cenário comparada com a densidade a posteriori (equação 2.12); (d) Histograma da amostra média gerada no quarto cenário comparada com a densidade a posteriori (equação 2.12) 


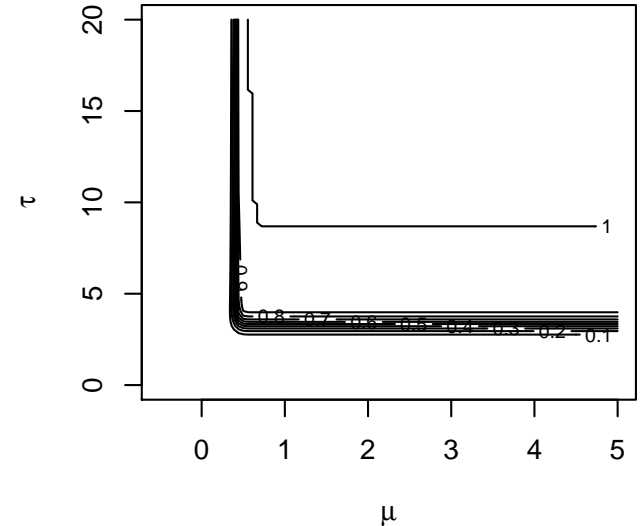

(a)

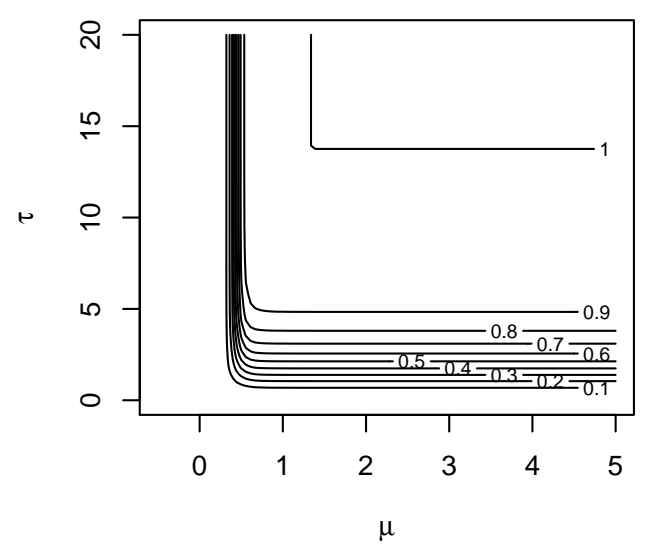

(c)

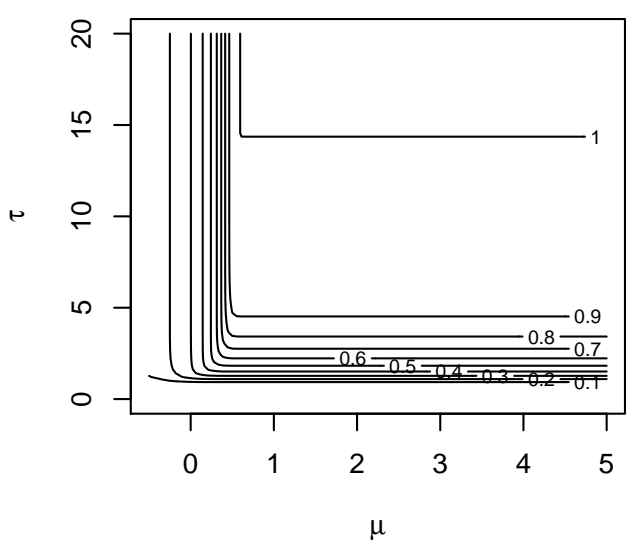

(b)

Figura 2.2: (a) Função de probabilidade acumulada de $(\mu, \tau) \mid \mathbf{x}$; (b) Função empírica média da amostra do primeiro cenário; (c) Função empírica média da amostra do segundo cenário. 


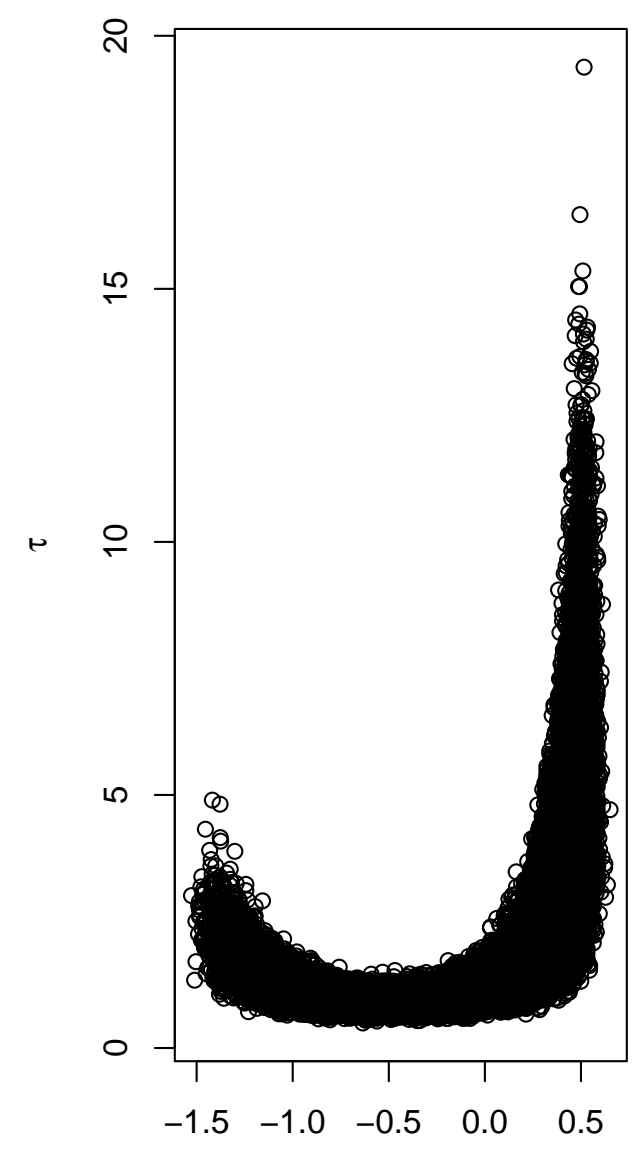

$\mu$

(a)

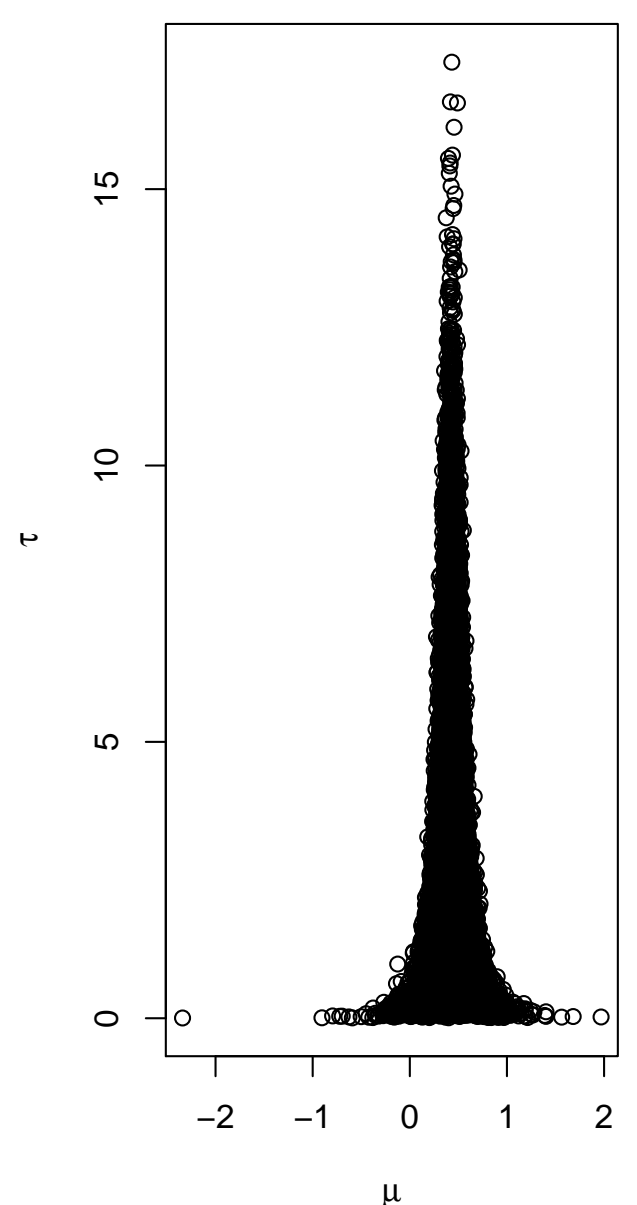

(b)

Figura 2.3: (a) Gráfico de dispersão de todas as amostras geradas no primeiro cenário; (b) Gráfico de dispersão de todas as amostras geradas no segundo cenário. 


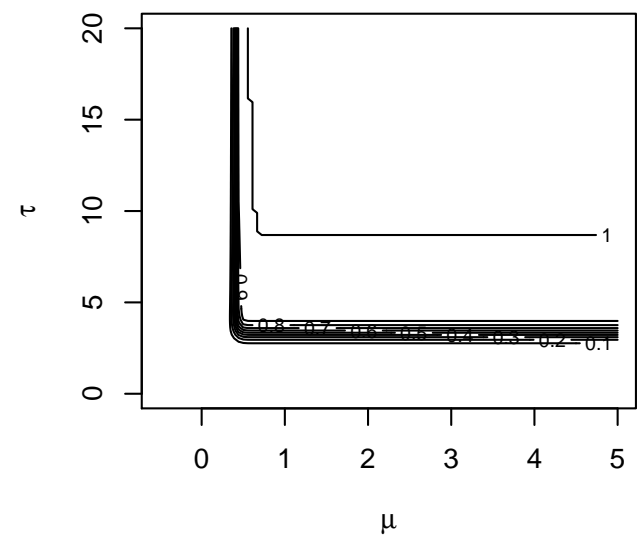

(a)

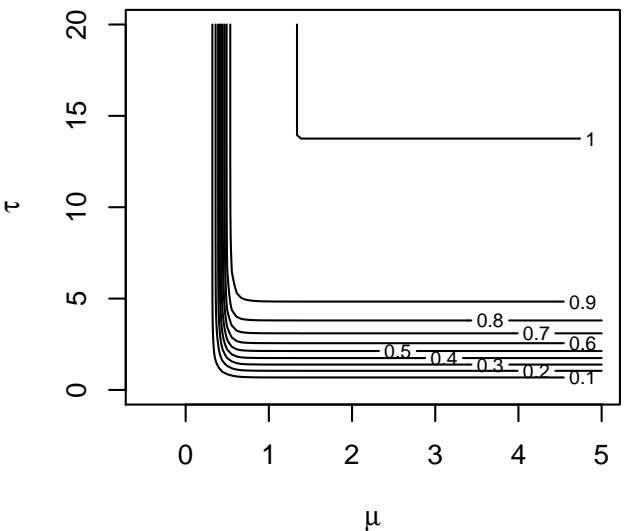

(c)

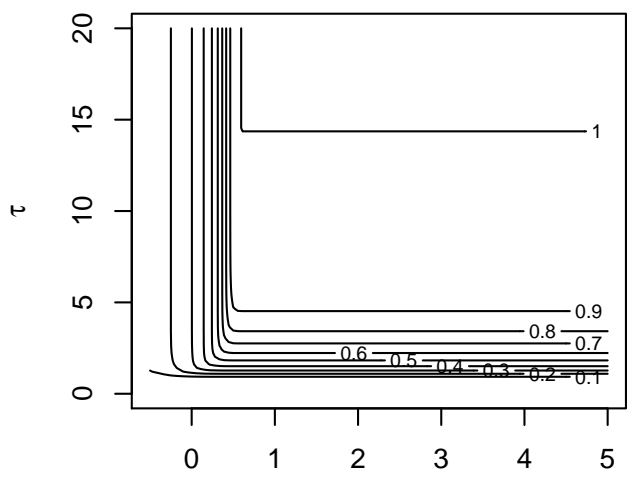

$\mu$

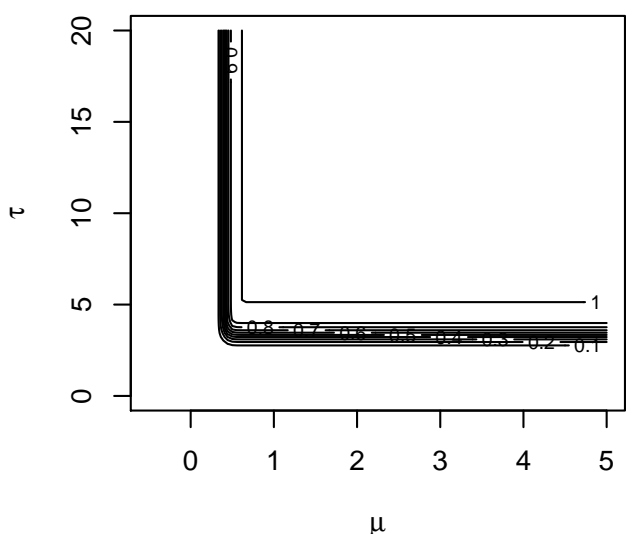

(d)

Figura 2.4: (a) Função de probabilidade acumulada de $(\mu, \tau) \mid \mathbf{x}$; (b) Função acumulada empírica média da amostra do primeiro cenário; (c) Função acumulada empírica média da amostra do segundo cenário; (d) Função acumulada empírica média da amostra gerada pelo amostrador de Gibbs. 


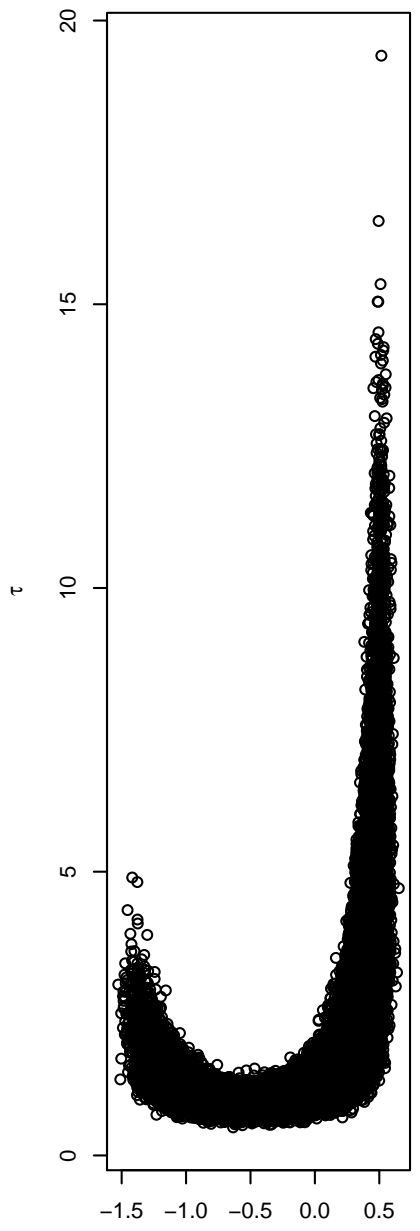

(a)

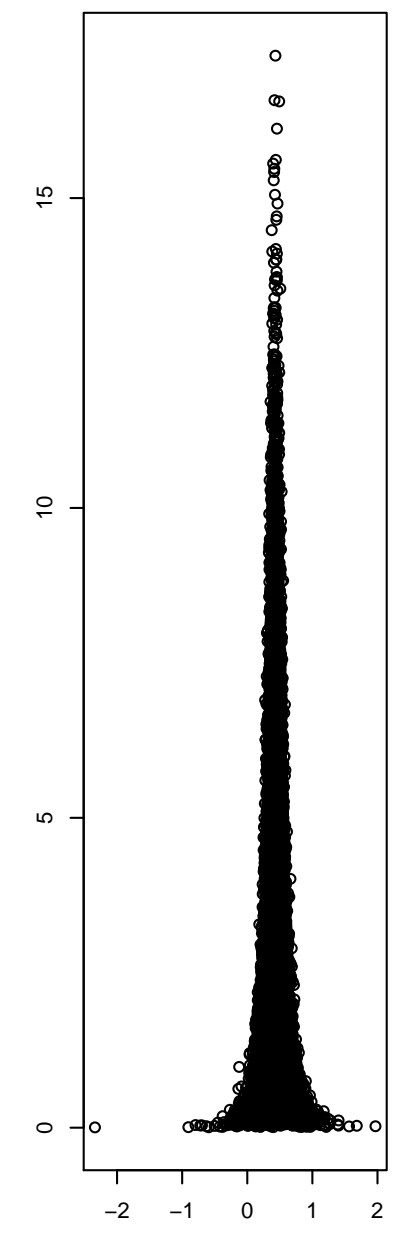

$\mu$
(b)

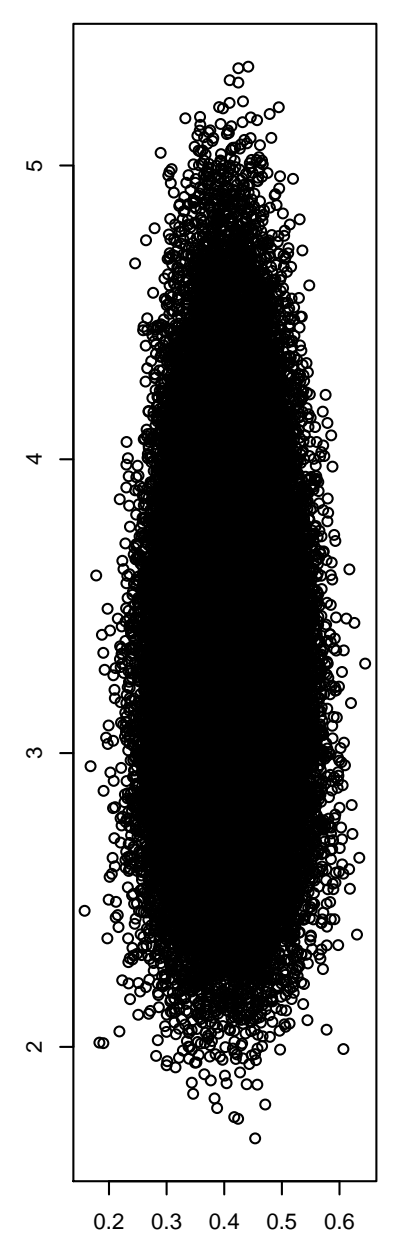

$\mu$
(c)

Figura 2.5: (a) Gráfico de dispersão de todas as amostras geradas no primeiro cenário; (b) Gráfico de dispersão de todas as amostras geradas no segundo cenário; (c) Gráfico de dispersão de todas as amostras geradas pelo amostrador de Gibbs. 


\section{Capítulo 3}

\section{Gerando observações de uma distribuição de Holgate}

\subsection{Introdução}

No presente capítulo é apresentado um outro Toy Model, mais complexo que os apresentados no capítulo anterior. Esse novo exemplo, considera os dados observados obedecendo a distribuição de Holgate. Essa complexidade é consequência de uma maior sofisticação matemática, que exemplifica de forma mais clara o fato de que o $\boldsymbol{A B C}$ não necessita da função de verossimilhança para gerar uma amostra de uma determinada distribuição a posteriori.

Esse grau complexidade, apresentada e analisada no decorrer desse capítulo, dificulta a implementação de algum algoritmo para gerar observações desta distribuição. Será mostrado como o algoritmo de Metropolis-Hastings (Gamerman e Lopes, 2006) pode ser usado para tal tarefa. Como nos exemplos passados, o $\boldsymbol{A} \boldsymbol{B} \boldsymbol{C}$ será posto em ação, e consequentemente, as amostras geradas por cada um dos dois algoritmos serão comparadas.

\subsection{Distribuição de Poisson bivariada}

Existem diferentes formas de representar a distribuição Poisson bivaraida. A que será tratada aqui, distribuição de Holgate, como dito em Chire (2013), é construído pelo método da redução trivariada. Basicamente, esse método consiste em reduzir a dimensão de um vetor, de três dimensões para duas, através de uma operação nas componentes do vetor original, aqui essa operação será a soma.

A construção de uma distribuição Holgate é feita da seguinte forma:

- Considere $U_{1}, U_{2}, U_{3}$ variáveis aleatórias (independentes) Poisson com parâmetros $\lambda_{1}, \lambda_{2}, \lambda_{3}$, respectivamente;

- Seja $X_{1}=U_{1}+U_{3}$ e $X_{2}=U_{2}+U_{3}$. O par $\left(X_{1}, X_{2}\right)$ tem distribuição Holgate com parâmetros $\left(\lambda_{1}, \lambda_{2}, \lambda_{3}\right)$ (ou $\left(\lambda_{1}+\lambda_{3}, \lambda_{2}+\lambda_{3}, \lambda_{3}\right)$ como visto em Stern e Zacks (2002));

- A função de probabilidade do par $\left(X_{1}, X_{2}\right)$ é da forma:

$$
P\left(X_{1}=x, X_{2}=y \mid \lambda_{1}, \lambda_{2}, \lambda_{3}\right)=e^{-\left(\lambda_{1}+\lambda_{2}+\lambda_{3}\right)} \sum_{i=0}^{\min (x, y)} \frac{\lambda_{1}^{x-i} \lambda_{2}^{y-i} \lambda_{3}^{i}}{(x-i) !(y-i) ! i !}
$$

Notem que, quando $U_{3}$ é igual a zero, as componentes do par $\left(X_{1}, X_{2}\right)$ são independentes. As distribuições de probabilidade marginais das variáveis $X_{1}$ e $X_{2}$ são da forma

$$
\begin{aligned}
& P\left(X_{1}=x \mid \lambda_{1}, \lambda_{3}\right)=\frac{e^{-\left(\lambda_{1}+\lambda_{3}\right)}\left(\lambda_{1}+\lambda_{3}\right)^{x}}{x !}, \\
& P\left(X_{2}=x \mid \lambda_{2}, \lambda_{3}\right)=\frac{e^{-\left(\lambda_{2}+\lambda_{3}\right)}\left(\lambda_{2}+\lambda_{3}\right)^{x}}{x !},
\end{aligned}
$$

i. e., palavras $X_{1} \sim \operatorname{Poisson}\left(\lambda_{1}+\lambda_{3}\right)$ e $X_{2} \sim \operatorname{Poisson}\left(\lambda_{2}+\lambda_{3}\right)$. Uma consequência desse fato é a covariância de $X_{1}$ e $X_{2}$ que é igual a $\lambda_{3}, \operatorname{COV}\left(X_{1}, X_{2}\right)=\lambda_{3}$ (Chire, 2013, pag. 11).

$$
\operatorname{COV}\left(X_{1}, X_{2}\right)=\operatorname{COV}\left(U_{1}+U_{3}, U_{2}+U_{3}\right)
$$




$$
\begin{aligned}
& =\operatorname{COV}\left(U_{1}+U_{3}, U_{2}\right)+\operatorname{COV}\left(U_{1}+U_{3}, U_{3}\right) \\
& =\operatorname{COV}\left(U_{1}+U_{2}\right)+\operatorname{COV}\left(U_{1}+U_{3}\right)+\operatorname{COV}\left(U_{3}, U_{2}\right)+\operatorname{COV}\left(U_{3}, U_{3}\right) \\
& =\operatorname{COV}\left(U_{3}, U_{3}\right) \\
& =\operatorname{Var}\left(U_{3}\right) \\
& =\lambda_{3} .
\end{aligned}
$$

Portanto $\lambda_{3}$ é uma médida de dependência entre $X_{1}$ e $X_{2}$ e,

$$
\rho_{X_{1}, X_{2}}=\frac{\lambda_{3}}{\sqrt{\left(\lambda_{1}+\lambda_{3}\right)\left(\lambda_{2}+\lambda_{3}\right)}} .
$$

Note que $0 \leq \rho_{X_{1}, X_{2}} \leq 1$, pois $\lambda_{1}, \lambda_{2}, \lambda_{3}>0$ e, além disso, a função de probabilidade do par $\left(X_{1}, X_{2}\right)$, quando $\rho_{X_{1}, X_{2}}=0$, é da seguinte forma,

$$
P\left(X_{1}=x, X_{2}=y\right)=\frac{\lambda_{1}^{x} \lambda_{2}^{y} e^{-\left(\lambda_{1}+\lambda_{2}\right)}}{x ! y !}, x, y=0,1, \ldots
$$

Considere agora um conjunto de observações independentes $\mathbf{x}=\left[\left(x_{1}, x_{2}\right)_{1},\left(x_{1}, x_{2}\right)_{2}, \ldots,\left(x_{1}, x_{2}\right)_{n}\right]^{\prime}$. A função de verossimilhança é dada da seguinte forma:

$$
L(\boldsymbol{\Lambda}, \mathbf{x})=e^{-n\left(\lambda_{1}+\lambda_{2}+\lambda_{3}\right)} \prod_{j=1}^{n} \sum_{i=0}^{\min \left(x_{j}, y_{j}\right)} \frac{\lambda_{1}^{x_{j}-i} \lambda_{2}^{y_{j}-i} \lambda_{3}^{i}}{\left(x_{j}-i\right) !\left(y_{j}-i\right) ! i !}
$$

onde $\boldsymbol{\Lambda}=\left(\lambda_{1}, \lambda_{2}, \lambda_{3}\right)$. Em Chire (2013) a função de verossimilhança é apresentada com uma diferente parametrização, em função das esperanças de $X_{1}$ e $X_{2}$. Sejam $\lambda_{1}^{*}=\lambda_{1}+\lambda_{3}=E\left[X_{1}\right]$ e $\lambda_{2}^{*}=\lambda_{2}+\lambda_{3}=E\left[X_{2}\right]$. A função de verossimilhança, parametrizada em função de $\boldsymbol{\Lambda}^{*}=\left(\lambda_{1}^{*}, \lambda_{2}^{*}, \lambda_{3}\right)$ é da forma:

$$
L\left(\boldsymbol{\Lambda}^{*}, \mathbf{x}\right)=e^{-n\left(\lambda_{1}+\lambda_{2}+\lambda_{3}\right)} \prod_{j=1}^{n} \sum_{i=0}^{\min \left(x_{j}, y_{j}\right)} \frac{\left(\lambda_{1}^{*}-\lambda 3\right)^{x_{j}-i}\left(\lambda_{2}^{*}-\lambda_{3}\right)^{y_{j}-i} \lambda_{3}^{i}}{\left(x_{j}-i\right) !\left(y_{j}-i\right) ! i !}
$$

Essa reparametrização, originalmente apresentada em Holgate (1964), de acordo com o próprio, torna o processo de estimação via máxima verossimilhança matematicamente mais simples, mas ainda assim continua bastante trabalhoso.

Todo o processo de estimação pontual, via método dos momentos e máxima verossimilhança, é apresentado em Chire (2013), bem como toda a metodologia para teste de hipóteses, via teste da razão de verossimilhança, referente a hipótese de independência entre $X_{1}$ e $X_{2}$, isto é, $\lambda_{3}=0$.

As estimativas pontuais de $\Lambda$, como mostradas em Chire (2013), via método dos momentos são as seguintes:

$$
\begin{aligned}
\widetilde{\lambda_{1}} & =\bar{X}_{1}-m_{1,1}, \\
\widetilde{\lambda_{2}} & =\bar{X}_{2}-m_{1,1}, \\
\widetilde{\lambda_{3}} & =m_{1,1},
\end{aligned}
$$

onde $m_{1,1}=\frac{1}{n} \sum_{(i, j) \in D}\left(i-\bar{X}_{1}\right)\left(j-\bar{X}_{2}\right) n_{i j}, n_{i j}=\sum_{k=1}^{n} \mathbb{I}_{\{(i, j)\}}\left(\left(X_{1}, X_{2}\right)_{k}\right)$ é a frequência de cada par $(i, j)$, para $i, j=0,1, \ldots$, e $D=\left\{(i, j): n_{i j}>0\right\}$ representa o conjunto de dados observados. Por consequência,

$$
n=\sum_{(i, j) \in D} n_{i j} .
$$

Já os estimadores via máxima verossimilhança, mais trabalhosos e complicados de serem obtidos, 
são:

$$
\begin{aligned}
& \widehat{\lambda_{1}}=\overline{X_{1}}-\widehat{\lambda_{3}}, \\
& \widehat{\lambda_{2}}=\bar{X}_{2}-\widehat{\lambda_{3}} .
\end{aligned}
$$

O estimador de máxima verossimilhança para $\lambda_{3}$ é obtido através da raiz da seguinte equação, com relação a $\widehat{\lambda_{3}}$,

$$
\frac{1}{n} \sum_{i=1}^{n} \frac{p\left(x_{i}-1, y_{i}-1\right)}{p\left(x_{i}, y_{i}\right)}=1
$$

utilizando $\widehat{\lambda_{1}^{*}}=\bar{X}_{1}$ e $\widehat{\lambda_{2}^{*}}=\bar{X}_{2}$, no intervalo $\left[0, \min \left(\bar{X}_{1}, \bar{X}_{2}\right)\right]$. A função $p\left(x_{i}, y_{i}\right)$ é a função de probabilidade $P\left(X_{1}=x_{i}, X_{2}=y_{i}\right)$ utilizando a reparametrização presente na equação 3.7;

\subsection{Abordagem Bayesiana}

Em Arruda (2000) há a necessidade de modelar, probabilisticamente, resultados de partidas de futebol, com a finalidade de poder prever os resultados das partidas seguintes, vitória, empate ou derrota do time com o mando de campo.

O placar do jogo pode ser encarado como uma variável aleatória de duas dimensões, com cada dimensão, marginalmente, seguindo uma distribuição univariada de Poisson. Portanto, pode-se considerar que esse vetor, aleatório, segue uma distribuição bivariada de Poisson. A distribuição de Holgate é uma possível distribuição de Poisson bivariada. Chire (2013) apresenta uma outra opção, a distribuição de Poisson generalizada bivariada.

O modelo Bayesiano abordado aqui, para estudar dados com essas características mencionadas acima, utiliza a distribuição de Holgate como distribuição dos dados observados, ou a serem observados.

Considere as seguintes distribuições de probabilidade, a priori, para $\left(\lambda_{1}, \lambda_{2}, \lambda_{3}\right)$.

$$
\begin{aligned}
& \lambda_{1} \sim \operatorname{Gama}\left(\alpha_{1}, \beta_{1}\right), \\
& \lambda_{2} \sim \operatorname{Gama}\left(\alpha_{2}, \beta_{2}\right), \\
& \lambda_{3} \sim \operatorname{Gama}\left(\alpha_{3}, \beta_{3}\right) .
\end{aligned}
$$

Considere agora que $\lambda_{1}, \lambda_{2}$ e $\lambda_{3}$ são, a priori, independentes, e, também, a seguinte parametrização da distribuição gama. Seja $x \sim \operatorname{Gama}(a, b)$, então:

$$
f_{X}(x)=\frac{b^{a}}{\Gamma(a)} x^{a-1} e^{-b x}, a>0, b>0, x>0 .
$$

Portanto, a distribuição a priori do trio ordenado $\left(\lambda_{1}, \lambda_{2}, \lambda_{3}\right)$ é

$$
\pi\left(\lambda_{1}, \lambda_{2}, \lambda_{3}\right) \propto \lambda_{1}^{\alpha_{1}-1} e^{-\beta_{1} \lambda_{1}} \lambda_{2}^{\alpha_{2}-1} e^{-\beta_{2} \lambda_{2}} \lambda_{3}^{\alpha_{3}-1} e^{-\beta_{3} \lambda_{3}} .
$$

Utilizando o teorema de Bayes com a priori, equação 3.17, e a função de verossimilhança, euqção 3.6, tem-se a seguinte distribuição de probabilidade a posteriori:

$$
\pi(\boldsymbol{\Lambda} \mid \mathbf{x}) \propto \lambda_{1}^{\alpha_{1}-1} \lambda_{2}^{\alpha_{2}-1} \lambda_{3}^{\alpha_{3}-1} e^{-\left(\lambda_{1}\left(n+\beta_{1}\right)+\lambda_{2}\left(n+\beta_{2}\right)+\lambda_{3}\left(n+\beta_{3}\right)\right)} \prod_{j=1}^{n} \sum_{i=0}^{m i n\left(x_{j}, y_{j}\right)} \frac{\lambda_{1}^{x_{j}-1} \lambda_{2}^{y_{j}-i} \lambda_{3}^{i}}{\left(x_{j}-1\right) !\left(y_{j}-i\right) ! i !} .
$$

Notem que o fator $\prod_{j=1}^{n} \sum_{i=0}^{\min \left(x_{j}, y_{j}\right)} \frac{\lambda_{1}^{x_{j}-1} \lambda_{2}^{y_{j}-i} \lambda_{3}^{i}}{\left(x_{j}-1\right) !\left(y_{j}-i\right) ! i !}$ é uma quantidade que, dificulta a obtenção da constante de normalização da distribuição a posteriori, e por consequência, deixa complexa qualquer tentativa de inferência da distribuição a posteriori.

É exatamente por esse motivo Chire (2013) apresenta uma abordagem alternativa, introduzindo 
variáveis latentes no modelo. Considere as variáveis $U_{1}, U_{2}$ e $U_{3}$ usadas para construir a distribuição de Holgate na seção 3.2 e o vetor aumentado $\left(X_{1}, X_{2}, U_{3}\right)$. A função de probabilidade deste vetor é dada por:

$$
\begin{aligned}
P\left(X_{1}=x, X_{2}=y, U_{3}=u \mid \boldsymbol{\Lambda}\right) & =P\left(U_{1}=x-u, U_{2}=y-u, U_{3}=u \mid \boldsymbol{\Lambda}\right) \\
& =\frac{\lambda_{1}^{x-u} \lambda_{2}^{y-u} \lambda_{3}^{u} e^{-\left(\lambda_{1}+\lambda_{2}+\lambda_{3}\right)}}{(x-u) !(y-u) ! u !}
\end{aligned}
$$

Note que $x, y=0,1, \ldots$ e $u=0,1, \ldots, \min (x, y)$.

Suponha uma amostra aleatória $D=\left\{\left(x_{1}, y_{1}, u_{1}\right), \ldots,\left(x_{n}, y_{n}, u_{n}\right)\right\}$. A função de verossimilhança baseada em $D$ é da seguinte forma:

$$
\begin{aligned}
L(D, \Lambda) & =\prod_{i=1}^{n} P\left(X_{1}=x_{i}, X_{2}=y_{i}, U_{3}=u_{i} \mid \Lambda\right) \\
& =\prod_{i=1}^{n} \frac{\lambda_{1}^{x_{i}-u_{i}} \lambda_{2}^{y_{i}-u_{i}} \lambda_{3}^{u_{i}} e^{-\left(\lambda_{1}+\lambda_{2}+\lambda_{3}\right)}}{\left(x_{i}-u_{i}\right) !\left(y_{i}-u_{i}\right) ! u_{i} !} \\
& \propto \lambda_{1}^{\sum_{i=1}^{n}\left(x_{i}-u_{i}\right)} e^{-n \lambda_{1}} \lambda_{2}^{\sum_{i=1}^{n}\left(y_{i}-u_{i}\right)} e^{-n \lambda_{2}} \lambda_{3}^{\sum_{i=1}^{n} u_{i}} e^{-n \lambda_{3}}
\end{aligned}
$$

Note que a função de verossimilhança 3.19 tem uma forma mais simples, e amigável, que a função de verossimilhança 3.6, pois não possui o um produtório de somatórios na sua expressão.

Utilizando o teorema de Bayes com a distribuição a priori 3.17 e a função de verossimilhança 3.19 tem-se a seguinte função densidade de probabilidade a posteriori:

$$
\pi(\Lambda \mid D) \propto \lambda_{1}^{\sum_{i=1}^{n}\left(x_{i}-u_{i}\right)+\alpha_{1}-1} e^{-\left(n+\beta_{1}\right) \lambda_{1}} \lambda_{2}^{\sum_{i=1}^{n}\left(y_{i}-u_{i}\right)+\alpha_{2}-1} e^{-\left(n+\beta_{2}\right) \lambda_{2}} \lambda_{3}^{\sum_{i=1}^{n} u_{i}+\alpha_{3}-1} e^{-\left(n+\beta_{3}\right) \lambda_{3}} .
$$

A densidade da distribuição a posteriori 3.20 tem uma forma analítica mais amigável que a densidade a posteriori 3.18, mas ainda assim é um problema para obter estimativas e realizar todo o processo inferencial de maneira direta, apenas utilizando a forma analítica densidade. Por isso há a necessidade de métodos que geram observações da distribuição de Holgate e assim realizar o processo inferencial tão desejados.

\subsection{Gerando observações}

Geralmente algoritmos MCMC são os primeiros que vêm a mente de um estatístico, e destes, o mais simples, de um certo ponto de vista, é o algoritmo de Gibbs (veja Casella e George, 1992).

\subsubsection{Gibbs Sampler}

Para implementar o algoritmo de Gibbs é necessário obter as formas analíticas das distribuições condicionais completas, e o mais importante, saber como gerar observações delas. A partir da distribuição a posteriori 3.18 as seguintes condicionais completas são obtidas:

$$
\begin{aligned}
& \pi\left(\lambda_{1} \mid \lambda_{2}, \lambda_{3}, \mathbf{x}\right) \propto \lambda_{1}^{\alpha_{1}-1} e^{-\lambda_{1}\left(n+\beta_{1}\right)} \prod_{j=1}^{n} \sum_{i=0}^{\min \left(x_{j}, y_{j}\right)} \frac{\lambda_{1}^{x_{j}-1} \lambda_{2}^{y_{j}-i} \lambda_{3}^{i}}{\left(x_{j}-1\right) !\left(y_{j}-i\right) ! i !} \\
& \pi\left(\lambda_{2} \mid \lambda_{1}, \lambda_{3}, \mathbf{x}\right) \propto \lambda_{2}^{\alpha_{2}-1} e^{-\lambda_{2}\left(n+\beta_{2}\right)} \prod_{j=1}^{n} \sum_{i=0}^{\min \left(x_{j}, y_{j}\right)} \frac{\lambda_{1}^{x_{j}-1} \lambda_{2}^{y_{j}-i} \lambda_{3}^{i}}{\left(x_{j}-1\right) !\left(y_{j}-i\right) ! i !} \\
& \pi\left(\lambda_{3} \mid \lambda_{1}, \lambda_{2}, \mathbf{x}\right) \propto \lambda_{3}^{\alpha_{3}-1} e^{-\lambda_{3}\left(n+\beta_{3}\right)} \prod_{j=1}^{n} \sum_{i=0}^{\min \left(x_{j}, y_{j}\right)} \frac{\lambda_{1}^{x_{j}-1} \lambda_{2}^{y_{j}-i} \lambda_{3}^{i}}{\left(x_{j}-1\right) !\left(y_{j}-i\right) ! i !}
\end{aligned}
$$

Novamente tem-se a complicação gerada pelo produto de somas. Devido a essa complicação não é possível obter a constante de normalização das distribuições condicionais completas. 
Os métodos numéricos de integração, utilizados para calcular a constante de normalização da condicionais completas, não obtiveram sucesso e, consequentemente, não foi possível obter-las, impossibilitando a geração observações de 3.21, 3.22 e 3.23 .

Chire (2013) apresenta uma configuração do amostrador de Gibbs utilizando a densidade 3.20, com dados aumentados. As distribuições condicionais completas são da forma:

$$
\begin{aligned}
\lambda_{1} \mid \lambda_{2}, \lambda_{3}, D & \sim \operatorname{Gama}\left(\sum_{i=1}^{n}\left(x_{i}-u_{i}\right)+\alpha_{1}, n+\beta_{1}\right) \\
\lambda_{2} \mid \lambda_{1}, \lambda_{3}, D & \sim \operatorname{Gama}\left(\sum_{i=1}^{n}\left(y_{i}-u_{i}\right)+\alpha_{2}, n+\beta_{2}\right) \\
\lambda_{3} \mid \lambda_{2}, \lambda_{1}, D & \sim \operatorname{Gama}\left(\sum_{i=1}^{n} u_{i}+\alpha_{3}, n+\beta_{3}\right) .
\end{aligned}
$$

Com

$$
P(u \mid x, y, \Lambda) \propto \prod_{i=1}^{n} \frac{1}{\left(x_{i}-u_{i}\right) !\left(y_{i}-u_{i}\right) ! u_{i} !}\left(\frac{\lambda_{3}}{\lambda_{2} \lambda_{1}}\right)^{u_{i}} .
$$

O algoritmo de Gibbs para gerar amostras da distribuição a posteriori da densidade 3.20, apresentado em Chire (2013), encontra-se a seguir.

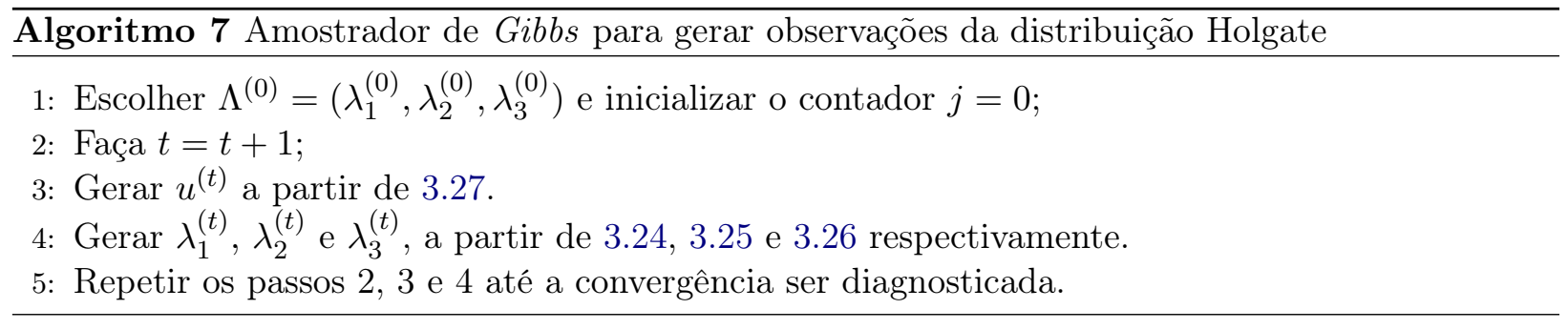

\subsubsection{Metropolis-Hastings}

O algoritmo de Metropolis-Hastings (Veja Gamerman e Lopes, 2006, cap. 6), no caso abordado neste capítulo, tem uma vantagem sobre o algoritmo de Gibbs. A despreocupação com o cálculo da constante de normalização das equações 3.213 .22 3.23. Essa vantagem é oriunda da própria estrutura do algoritmo.

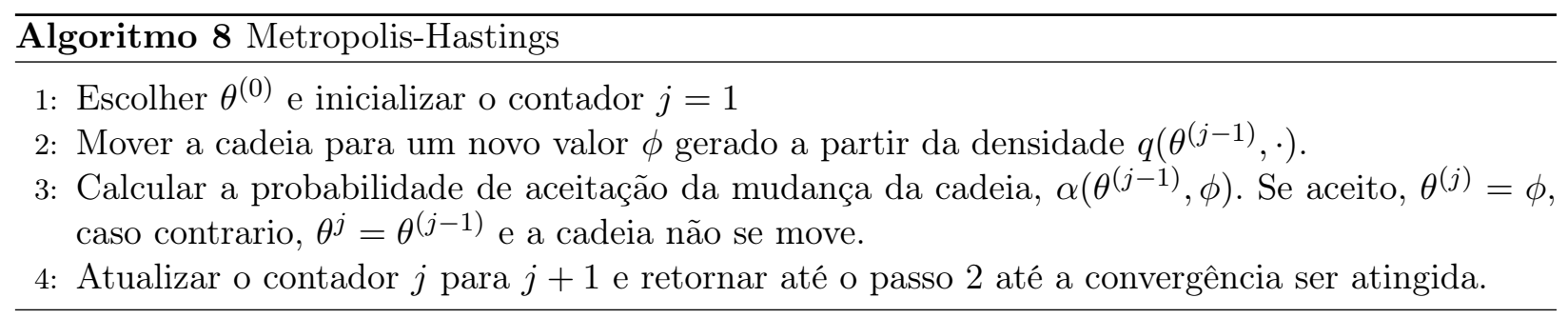

A quantidade $\alpha\left(\theta^{(j-1)}, \phi\right)$ do algoritmo 8 representa a probabilidade de que $\phi$ seja aceito como um novo elemento da trajetória da cadeia de Markov, sendo calculado pela seguinte expressão:

$$
\alpha(\theta, \phi)=\min \left\{1, \frac{\pi(\phi) q(\phi, \theta)}{\pi(\theta) q(\theta, \phi)}\right\} .
$$

A função $q(\theta, \phi)$ é chamada de kernel de transição, uma versão da matriz de transição(espaço de estados discreto) para quando o espaço de estados é contínuo. Representa, como o próprio nome já diz, a transição da cadeia de Markov do estado $\theta$ para o estado $\phi$. A função $\pi(\cdot)$ representa a densidade alvo, da qual há interesse em gerar uma amostra. Como há uma razão de funções, $\pi(\phi) / \pi(\theta)$, tudo o que não depender de $\phi$ e $\theta$ será simplificado, incluindo a constante de normalização. 
Dentre os casos especiais do algoritmo de Metropolis-Hastings apresentados em Gamerman e Lopes (2006) foram geradas amostras de teste usando os caso especiais Random Walk Chain e Independence Chain.

Para implementar o algoritmo de Metropolis-Hastings, através da técnica Random Walk Chain os resultados das equações 3.21 a 3.23 foram aproveitados, decidiu-se evoluir uma cadeia para cada um dos $\lambda_{i}$. Uma cadeia influenciará no na outra através do calculo de $\alpha(\theta, \phi)$.

Como comentado em Gamerman e Lopes (2006), no algoritmo de Metropolis-Hastings via random walk chain, a evolução da cadeia de Markov ocorre da seguinte forma:

$$
\theta^{(j)}=\theta^{(j-1)}+w_{j},
$$

onde $w_{j}$ é uma variável aleatória com distribuição independente da cadeia. Geralmente as variáveis $w_{j}$ são independentes e identicamente distribuídas com densidade $f_{w}$. Deste modo o kernel de transição para este caso especial é $q(\theta, \phi)=f_{w}(\phi-\theta)$.

Decidiu-se criar uma cadeia de Markov para cada um $\operatorname{dos} \lambda^{\prime} s$ e, portanto, foram definidas três distribuições para os ruídos, $w_{j}^{\left(\lambda_{1}\right)} \sim \operatorname{Normal}\left(0, \nu \frac{\alpha_{1}+\sum_{i=1}^{n} x_{i}}{\left(\beta_{1}+n\right)^{2}}\right), w_{j}^{\left(\lambda_{2}\right)} \sim \operatorname{Normal}\left(0, \nu \frac{\alpha_{2}+\sum_{i=1}^{n} y_{i}}{\left(\beta_{2}+n\right)^{2}}\right)$ e $w_{j}^{\left(\lambda_{3}\right)} \sim \operatorname{Normal}\left(0, \nu \frac{\alpha_{3}+\operatorname{cov}(x, y)}{\left(\beta_{3}+n\right)^{2}}\right)$, onde $\nu$ é um parâmetro de tunning que ajusta a velocidade de convergência em oposição a taxa de aceitação do algoritmo. As variâncias das distribuições dos ruídos foram escolhidas da maneira apresentada acima, pelo motivo de restringir o espaço de variação das cadeias, baseando-se na amostra observada. Espera-se que isso melhore o desempenho computacional do algoritmo.

Pelo fato das distribuições de $w_{j}^{\left(\lambda_{1}\right)}, w_{j}^{\left(\lambda_{2}\right)}$ e $w_{j}^{\left(\lambda_{1}\right)}$ serem simétricas em torno do valor zero, há uma simetria nas cadeias de Markov, portanto, $q_{\lambda_{i}}(\theta, \phi)=q_{\lambda_{i}}(\phi, \theta)$ e $\alpha_{\lambda_{i}}(\theta, \phi)=\min \left\{1, \frac{\pi_{\lambda_{i}}(\phi)}{\pi_{\lambda_{i}}(\theta)}\right\}$, onde as distribuições $\pi_{\lambda_{i}}(\cdot)$ referem-se as equações 3.21 a 3.23 .

A outra técnica explorada foi o Metropolis-Hastings via Independence Chain, onde o estado atual da cadeia foi gerado de forma independente do estado anterior. Como Gamerman e Lopes (2006) explica, apesar da cadeia evoluir com independência entre os estados, o algoritmo de MetropolisHastings ainda apresenta a propriedade markoviana, pois a probabilidade, $\alpha(\theta, \phi)$, de aceitar ou não um valor como parte da cadeia no atual instante, ainda depende do estado da cadeia no instante anterior.

Para implementar o algoritmo de Metropolis-Hastings via Independence decidiu-se evoluir uma cadeia tridimensional para o trio ordenado $\Lambda=\left(\lambda_{1}, \lambda_{2}, \lambda_{3}\right)$, da seguinte maneira:

$$
f_{\Lambda}\left(l_{1}, l_{2}, l_{3}\right)=\frac{\beta_{1}^{\alpha_{1}}}{\Gamma\left(\alpha_{1}\right)} l_{1}^{\alpha_{1}-1} e^{-\beta_{1} l_{1}} \frac{\beta_{2}^{\alpha_{2}}}{\Gamma\left(\alpha_{2}\right)} l_{2}^{\alpha_{2}-1} e^{-\beta_{2} l_{2}} \frac{\beta_{3}^{\alpha_{3}}}{\Gamma\left(\alpha_{3}\right)} l_{3}^{\alpha_{3}-1} e^{-\beta_{3} l_{3}}
$$

Deste modo, $\alpha_{\Lambda}(\theta, \phi)=\min \left\{1, \frac{\pi_{\Lambda}(\phi) \prod_{i=1}^{3} \theta^{\alpha_{i}-1} e^{-\theta \beta_{i}}}{\pi_{\Lambda}(\theta) \prod_{i=1}^{3} \phi^{\alpha_{i}-1} e^{-\phi \beta_{i}}}\right\}$, onde $\pi_{\Lambda}(\cdot)$ refere-se a distribuição 3.18 .

O critério de convergência adotado será especificado mais adiante, mas pode-se adiantar que baseia-se na média ergódica da cadeia. Se a média ergódica manter-se, com um certo nível de tolerância, estável por um tempo razoavelmente longo, considera-se que a cadeia convergiu.

Os pseudo-códigos usados para implementar o Random Walk Chain Metropolis-Hastings e Independence Chain Metropolis-Hastings são apresentados a seguir, algoritmos 9 e 10

\subsection{3 $A B C$}

Os detalhes sobre o funcionamento do $\boldsymbol{A} B \boldsymbol{C}$ já foram expostos no capítulo 2, discutindo-se aqui apenas adaptações especificas do $\boldsymbol{A} \boldsymbol{B} \boldsymbol{C}$ para gerar observações para o modelo da distribuição Holgate.

Apesar de observar uma variável de contagem-placar de um jogo-foi implementada a versão do $\boldsymbol{A} \boldsymbol{B} \boldsymbol{C}$ para variáveis contínuas, algoritmo 2. Tal fato é motivado por critérios computacionais. Em simulações preliminares foi constatado que o algoritmo necessitava de um número bastante elevado de iterações para poder aceitar um elemento gerado como parte de uma amostra de interesse. Ao 

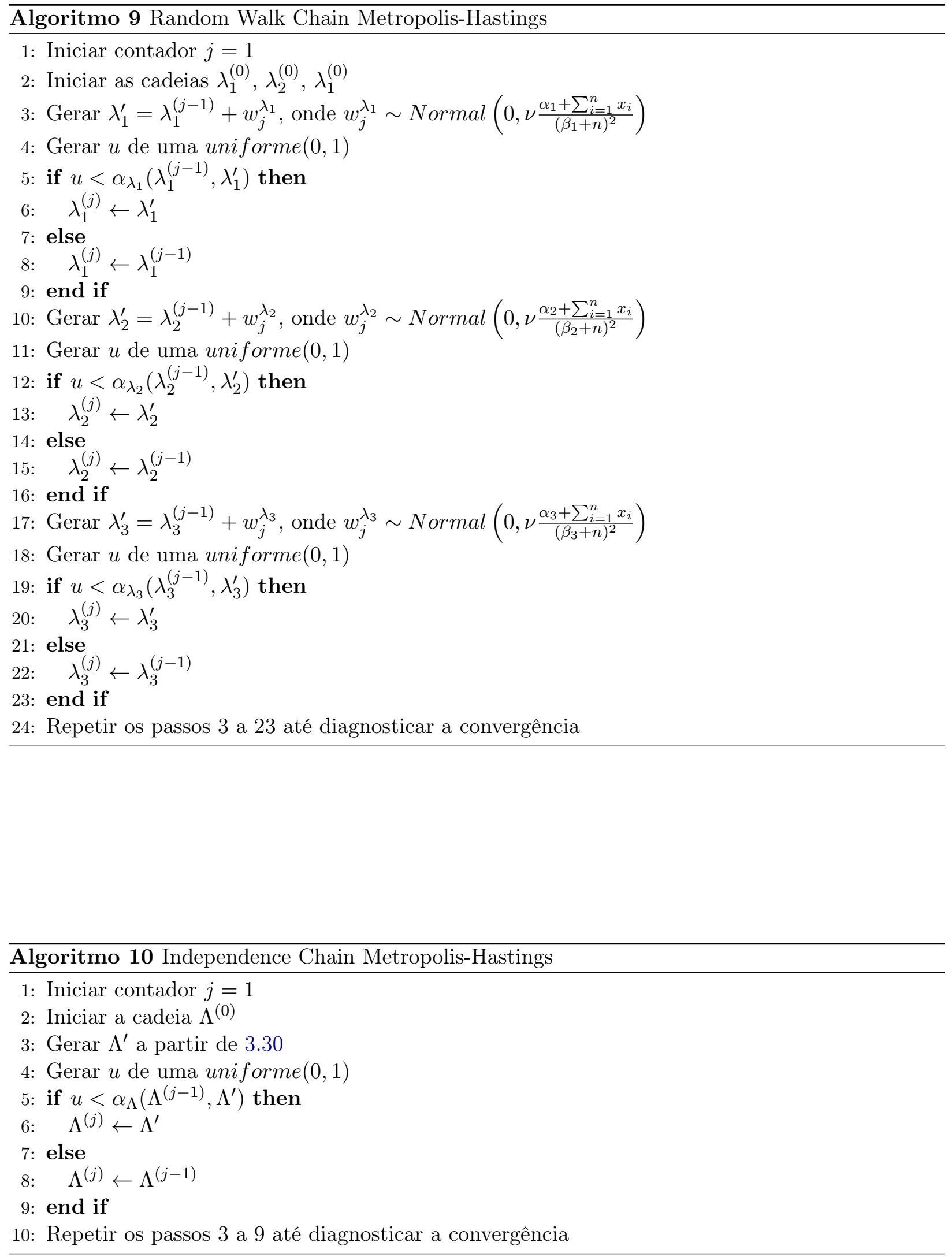
usar a versão do $\boldsymbol{A B C}$ para variáveis contínuas, a taxa de rejeição diminui, poupando assim recursos computacionais, em detrimento da qualidade da amostra gerada. Espera-se que alguns pontos aceitos pelo $\boldsymbol{A} \boldsymbol{B} \boldsymbol{C}$-Rejeição, para variáveis contínuas, não sejam aceitos pela versão do $\boldsymbol{A} \boldsymbol{B} \boldsymbol{C}$-Rejeição para variáveis discretas, o que implica numa aproximação não tão boa quanto a desejada.

O vetor de sumário de amostra será um vetor com a primeira componente igual à média das primeiras componentes das amostras. A segunda componente do vetor de sumário é a média das segundas componentes da amostra, e por fim, a última componente do vetor de sumário é a covariância da primeira e segunda componentes da $\operatorname{amostra}\left(\eta(\mathbf{x})=\left(\overline{\mathbf{x}}_{1, \cdot}, \overline{\mathbf{x}}_{2, .}, \operatorname{cov}\left(\mathbf{x}_{1, \cdot}, \mathbf{x}_{2, \cdot}\right)\right)\right)$. Tal escolha foi motivada pela facilidade em obter tais quantidades, sendo mais simples de calcular que os estimadores de máxima verossimilhança e método dos momentos.

Note que nesse toy model $\eta(\mathbf{x})$ não é uma estatística suficiente para $\left(\lambda_{1} \cdot \lambda_{2}, \lambda_{3}\right)$. A tarefa de encontrar um estimador suficiente para $\Lambda$ não é nada simples, tendo em vista a complexidade da função de probabilidade,e por consequência da função de verossimilhança.

Definiu-se a função distância $\rho(\cdot)$ como sendo a distancia euclidiana entre os dois vetores de sumário, o da amostra observada $(x)$ e o da amostra gerada $(y)$.

O algoritmo $\boldsymbol{A} \boldsymbol{B C}$, configurado para gerar amostras de uma distribuição de Holgate está apresentado a seguir, algoritmo 11

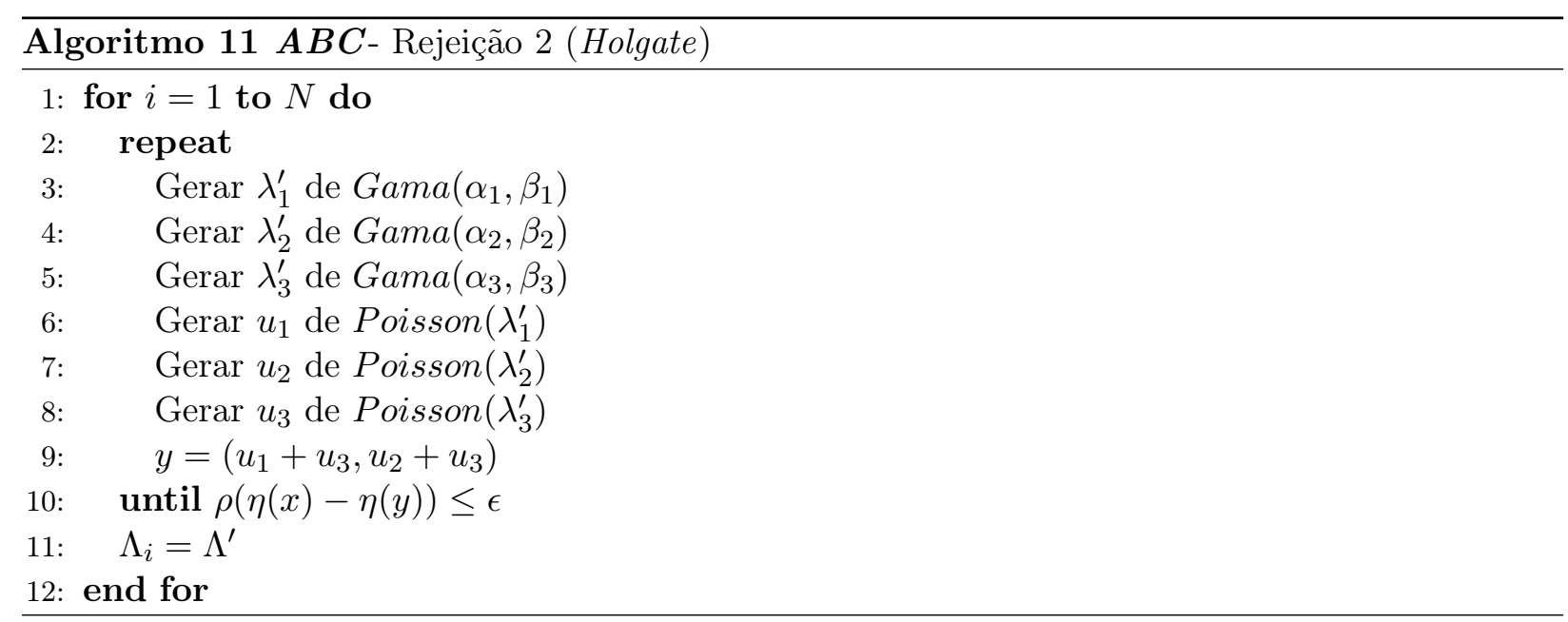

\subsubsection{Comparando métodos}

Com a finalidade de comparar o desempenho dos três algoritmos, 9, 10 e 11, foram geradas observações da mesma distribuição a posteriori.

Para tal distribuição, foram escolhidos os hiperparâmetros da seguinte forma: $\left(\alpha_{1}, \alpha_{2}, \alpha_{3}\right)=$ $\left(\beta_{1}, \beta_{2}, \beta_{3}\right)=(1,1,1)$. Como amostra observada, $\mathbf{x}$ considere a sequência de pares encontrados em Arruda (2000, pag. 12),

$$
\mathbf{x}=((1,0),(1,2),(2,3),(3,2),(3,0),(2,1)),
$$

considere também que tal amostra consiste de observações permutáveis.

O critério usado para diagnosticar a convergência das cadeias de Markov, algoritmos 9 e 10, foi usar a média ergódica da cadeia. No algoritmo que utiliza a metodologia de passeio aleatório as, três cadeias, uma para cada $\lambda_{i}$, as três médias ergódicas devem se manter iguais, com uma tolerância de $10^{-5}$, por 500 iterações. Já o algoritmo que usa a metodologia de cadeias independentes, a média ergódica da cadeia, referente a $\Lambda$ deve se manter igual com uma tolerância de $10^{-5}$, por 500 iterações.

O $\boldsymbol{A B C}$ implementado para o modelo Holgate não necessita de um critério de convergência, mas necessita de uma tolerância, o quanto a amostra gerada está próxima da amostra observada. Sabemos que essa tolerância influencia na velocidade do algoritmo e pensando nisso foi imposta uma tolerância de $10^{-5}$ para manter uma certa justiça entre os algoritmos trabalhados, mas em 
testes preliminares usar um $\epsilon$ tão pequeno revelou-se impraticável. Este fato expõe uma fraqueza do $\boldsymbol{A} \boldsymbol{B} \boldsymbol{C}$. Por fim houve a necessidade da escolher um $\epsilon$ maior. Neste caso, a tolerância escolhida foi $10^{-2}$.

O amostrador de Gibbs não foi implementado por dois motivos. O primeiro é que ele já é implementado e analisado em Chire (2013). O segundo motivo é que ainda não é claro como gerar valores da distribuição de $u \mid x, y, \Lambda$, equação 3.27. Foi considerado que a complexidade de gerar observações de $u \mid x, y, \Lambda$ é equivalente a efetuar cálculos na função de verossimilhança 3.6.

Para os três algoritmos foram geradas 10 amostras de tamanho 1000. Para os algoritmos baseados em $M C M C$, a cada observação gerada, era iniciada uma nova cadeia, com estados iniciais gerados aleatoriamente, para assegurar a independência das observações da amostra final.

Algumas estatísticas descritivas do número de iterações que cada algoritmo levou para gerar uma observação do modelo Holgate estão dispostas na tabela 3.1. É possível ver que a metodologia MCMC, baseada em passeio aleatório, aparenta ser a mais eficiente na geração de amostras, pois é a metodologia mais rápida, necessitando de menos iterações, seguida pela metodologia do $\boldsymbol{A} B \boldsymbol{C}$ e do MCMC, com em cadeias independentes.

Tabela 3.1: Sumário do número de iterações necessárias para gerar uma observação da distribuição de Holgate usando os algoritmos 9, 10 e 11

\begin{tabular}{lccccccc}
\hline \hline & Mínimo & $1^{\circ}$ Quartil & Mediana & Média & $3^{\circ}$ Quartil & Máximo & Variância \\
\hline Random Walk MCMC & 508 & 547 & 560 & 564,2 & 575 & 371 & 634,592 \\
\hline Independence Chain MCMC & 9588 & 15489 & 19648 & 21689 & 26214 & 55365 & 60560197 \\
\hline $\boldsymbol{A B C}$ & 1 & 1500 & 3688 & 5409 & 7456 & 57340 & 30582628 \\
\hline \hline
\end{tabular}

Os valores presente na coluna variância da tabela 3.1 podem parecer confusos. Como a variância do número de iterações do algoritmo $\boldsymbol{A} \boldsymbol{B} \boldsymbol{C}$ pode ser maior que a variância número de iterações do algoritmo Independence Chain $M C M C$, a amplitude do número de iterações do $\boldsymbol{A} \boldsymbol{B} \boldsymbol{C}$ é maior? A resposta para essa pergunta encontra-se nas informações que podem ser extraídas no gráfico da figura 3.1.

É possível ver que $75 \%$ das iterações do $\boldsymbol{A B C}$ foram menores que o valor mínimo das iterações do Independence Chain $M C M C$. Deste modo, a ordem de grandeza do número de iterações do Independence Chain $M C M C$ é bem maior que a ordem de grandeza do número de iterações do $\boldsymbol{A} \boldsymbol{B C}$, o que explica o fato da variância do número da iterações do $\boldsymbol{A} \boldsymbol{B} \boldsymbol{C}$ quase a metade da variância do número da iterações do Independence Chain $M C M C$, mesmo tendo uma amplitude maior.

Na figura 3.2 estão apresentados os histogramas de $\lambda_{1}, \lambda_{2}$ e $\lambda_{3}$, cujas amostras foram geradas pela metodologia de MCMC via passeio aleatório. As 10 amostras de tamanho 1000 foram consideradas como uma única amostra de tamanho 10000, como feito nos toy models do capítulo 2. Percebeu-se que há uma quantidade demasiadamente grande de observações na cauda direita dos histogramas, indicando uma bimodalidade das distribuições.

Este comportamento bimodal dos histogramas pode ser indício de uma dependência entre $\lambda_{1}$, $\lambda_{2}$ e $\lambda_{3}$, em que $\lambda_{2}$ e $\lambda_{3}$ dependem de $\lambda_{1}$, o que contraria as crenças a priori sobre $\Lambda$. Não existem motivos para acreditar que $\lambda_{1}, \lambda_{2}$ e $\lambda_{3}$ apresentem uma dependência a posteriori.

Uma outra explicação para este fato advém da taxa de aceitação do algoritmo. Durante o tempo em que o algoritmo 9 estava em execução percebeu-se que as cadeias passeavam algumas regiões especificas, restringindo sua variação a essas regiões, ocasionando poucas mudanças na trajetória da cadeia.

Por fim, o estimador de Bayes, considerando essa amostra, de $\Lambda=\left(\lambda_{1}, \lambda_{2}, \lambda_{3}\right)$, sob perda quadrática, é $\widehat{\Lambda}=(0.50390,1.66900,2.13800)$.

Já na figura 3.3 observamv-se os histogramas de $\lambda_{1}, \lambda_{2}$ e $\lambda_{3}$, considerando a amostra gerada pelo algoritmo 10. É possível ver que neste caso já não é constatada a bimodalidade nos histogramas de $\lambda_{2}$ e $\lambda_{3}$ que são apresentados na figura 3.2, o que pode indicar que esse fenômeno pode ter acontecido devido ao fato de que no algoritmo 9 atualiza primeiro a cadeia referente a $\lambda_{1}$ e depois as cadeias 
subsequentes, já com o algoritmo 10 há apenas uma cadeia sendo evoluída, e assim $\lambda_{1}, \lambda_{2}$ e $\lambda_{3}$ são atualizados conjuntamente, não havendo a possibilidade de uma componente de $\left(\lambda_{1}, \lambda_{2}, \lambda_{3}\right)$ ficar estagnada em um estado específico.

Os histogramas apresentam um comportamento semelhante ao de distribuição Gama, o que está dentro do esperado, já que a distribuição a priori de $\Lambda$ é uma distribuição trivariada cujas distribuições marginais são Gamas independentes. Finalizando os comentários sobre a amostra gerada pelo algoritmo 10 é apresentada a estimativa de Bayes, com relação a perda quadrática, de $\Lambda$ como sendo o vetor $(0.6417,0.638200,0.7361)$.

Por fim, na figura 3.4 estão os histogramas da amostra, de tamanho 10000, a posteriori de $\lambda_{1}, \lambda_{2}$ e $\lambda_{3}$ gerada pelo algoritmo 11 . Nota-se que há comportamento que lembra uma distribuição Gama para os três histogramas, como na figura 3.3, o que já era esperado. O vetor de estimativas de Bayes, a posteriori, com relação a perda quadrática, para $\left(\lambda_{1}, \lambda_{2}, \lambda_{3}\right)$ é $(1.318548,0.7509752,0.6750414)$, respectivamente. Pode-se considerar que tanto este vetor de estimativas, quanto as estimativas da amostra apresentada na figura 3.3, são estimativas plausíveis pra $\Lambda$.

Perceba que as amostras gerada pelo $\boldsymbol{A} \boldsymbol{B} \boldsymbol{C}$ são mais dispersas que as amostras geradas pelo Metropolis-Hastings via Random Walk Chain, mais um fato que pode ser atribuído à tendência que o $\boldsymbol{A} \boldsymbol{B} \boldsymbol{C}$-Rejeição tem de gerar observações em regiões com baixa probabilidade posteriori. Comparando as amostras geradas por esses dois métodos, com a amostra gerada pelo algoritmo de Gibbs, apresentado em Chire (2013, pag. 53), pode-se constatar que as dua amostras são coerentes, salvo as mudanças nos hiperparâmetros.

Como as amostras geradas pelo $\boldsymbol{A B C}$ e pelo Independence Chain $M C M C$ se mostraram bem semelhantes, marginalmente, decidiu-se por construir os histogramas em três dimensões dos $\lambda^{\prime} s$, dois a dois, gerados por cada um dos métodos. Nas figuras 3.5, 3.6 e 3.6 estão representados os histogramas, em três dimensões, dos $\lambda^{\prime} s$ gerados pelo Independence Chain MCMC. E nas figuras 3.8, 3.9 e 3.9 estão representados os histogramas, em três dimensões, dos $\lambda^{\prime} s$ gerados pelo $\boldsymbol{A} \boldsymbol{B} \boldsymbol{C}$. Ao comparar os histogramas, de duas e três dimensões, das amostras geradas pelos dois métodos, é possível ver, novamente, que as amostras geradas pelo $A B C$ são mais dispersas do que as geradas pelos métodos mais usuais, refletindo a tendencia, possuída pelo $\boldsymbol{A B C}$-Rejeição, de gerar pontos em regiões de baixa probabilidade posteriori.

\subsection{Prevendo novas observações}

O passo final do processo de inferência é a predição de observações futuras, dado as atuais observações. Seja $P\left(X_{n+1} \mid X_{n}=x_{n}, \ldots, X_{1}=x_{1}\right)$ a distribuição de probabilidade preditiva, a distribuição de probabilidade que ditará a probabilidade do que acontecerá em futuras realizações do experimento de interesse. Tal distribuição é obtida da seguinte forma:

$$
P\left(X_{n+1} \mid X_{n}=x_{n}, \ldots, X_{1}=x_{1}\right)=\int_{\theta} P_{X}\left(X_{n+1} \mid \theta\right) \pi\left(\theta \mid X_{n}=x_{n}, \ldots, X_{1}=x_{1}\right) d \theta,
$$

onde $P_{X}(\cdot \mid \theta)$ representa o modelo probabilístico imposto à variável aleatória observada.

De uma maneira simples, pode-se escrever

$$
P\left(X_{n+1} \mid X_{n}=x_{n}, \ldots, X_{1}=x_{1}\right)=E\left[P_{X}\left(X_{n+1} \mid \theta\right)\right] .
$$

Note que a esperança é calculada levando em consideração a distribuição de $\theta \mid X_{n}=x_{n}, \ldots, X_{1}=x_{1}$.

Quando considera-se que os dados seguem a distribuição de Holgate, equação 3.1, tem-se:

$$
\begin{aligned}
P\left(X_{1_{n+1}}=x, X_{2_{n+1}}=y \mid\left(x_{1}, x_{2}\right)_{n}, \ldots,\left(x_{1}, x_{2}\right)_{1}\right) & =E\left[P_{\left(X_{1}, X_{2}\right)}\left(X_{1}=x, X_{2}=y \mid \lambda_{1}, \lambda_{2}, \lambda_{3}\right)\right] \\
& =E\left[e^{-\left(\lambda_{1}+\lambda_{2}+\lambda_{3}\right)} \sum_{i=0}^{\min (x, y)} \frac{\lambda_{1}^{x-i} \lambda_{2}^{y-i} \lambda_{3}^{i}}{(x-i) !(y-i) ! i !}\right]
\end{aligned}
$$

com

$$
\pi\left(\lambda_{1}, \lambda_{2}, \lambda_{3}\right) \propto \lambda_{1}^{\alpha_{1}-1} e^{-\beta_{1} \lambda_{1}} \lambda_{2}^{\alpha_{2}-1} e^{-\beta_{2} \lambda_{2}} \lambda_{3}^{\alpha_{3}-1} e^{-\beta_{3} \lambda_{3}}
$$


$\mathrm{e}$

$$
\pi\left(\lambda_{1}, \lambda_{2}, \lambda_{3} \mid \mathbf{x}\right) \propto \lambda_{1}^{\alpha_{1}-1} \lambda_{2}^{\alpha_{2}-1} \lambda_{3}^{\alpha_{3}-1} e^{-\left(\lambda_{1}\left(n+\beta_{1}\right)+\lambda_{2}\left(n+\beta_{2}\right)+\lambda_{3}\left(n+\beta_{3}\right)\right)} \prod_{j=1}^{n} \sum_{i=0}^{\min \left(x_{j}, y_{j}\right)} \frac{\lambda_{1}^{x_{j}-1} \lambda_{2}^{y_{j}-i} \lambda_{3}^{i}}{\left(x_{j}-1\right) !\left(y_{j}-i\right) ! i !} .
$$

Calcular $P\left(X_{1_{n+1}}=x, X_{2_{n+1}}=y \mid\left(x_{1}, x_{2}\right)_{n}, \ldots,\left(x_{1}, x_{2}\right)_{1}\right)$ é uma tarefa bastante complicada, quase inviável realiza-la de forma analítica, portanto decidiu-se por utilizar as amostras geradas pelo $\boldsymbol{A} \boldsymbol{B} \boldsymbol{C}$ e calcular $E\left[P_{\left(X_{1}, X_{2}\right)}\left(X_{1}=x, X_{2}=y \mid \lambda_{1}, \lambda_{2}, \lambda_{3}\right)\right]$ de forma numérica.

A quantidade $E\left[P_{\left(X_{1}, X_{2}\right)}\left(X_{1}=x, X_{2}=y \mid \lambda_{1}, \lambda_{2}, \lambda_{3}\right)\right]$ foi obtida calculando

$$
\frac{1}{m} \sum_{i=1}^{m} P_{\left(X_{1}, X_{2}\right)}\left(X_{1}=x, X_{2}=y \mid\left(\lambda_{1}, \lambda_{2}, \lambda_{3}\right)_{i}\right)
$$

para um par $(x, y)$ determinado e todos os trios $\lambda^{\prime} s$ gerados pelo $\boldsymbol{A} \boldsymbol{B C}$. Fazendo esse calculo para todos os possíveis pares $(x, y)$ tem-se a distribuição de probabilidade preditiva para uma nova observação.

Em Arruda (2000), a predição de novos placares não é feita usando a função de probabilidade preditiva pelo fato dos placares observados não formarem uma amostra permutável. Os trios de $\left(\lambda_{1}, \lambda_{2}, \lambda_{3}\right)$ não eram os mesmos, por suposição, para os placares observados.

Considerando a amostra presente em Arruda $(2000)(\mathbf{x}=(1,0),(1,2),(2,3),(3,2),(3,0),(2,1))$ como permutáveis, com a finalidade de ilustrar a distribuição preditiva.

Realizando a todo o processo, já comentado aqui, com a amostra, já apresentada aqui, a distribuição de probabilidade preditiva, para quando são observados vetores aleatórios seguindo a distribuição de Holgate, truncada no reticulado $\{0,1,2,3,4,5,6\} \times\{0,1,2,3,4,5,6\}$ está representada na tabela abaixo, tabela 3.2. Os valores foram arredondados para duas casas decimais, portanto, os valores iguais a zero na tabela são valores pequenos, menores que $10^{-2}$.

Tabela 3.2: Distribuição preditiva

\begin{tabular}{cccccccc}
\hline \hline$X \backslash Y$ & 1 & 2 & 3 & 4 & 5 & 6 & 7 \\
\hline 1 & 0.08 & 0.05 & 0.02 & 0.01 & 0.00 & 0.00 & 0.00 \\
2 & 0.09 & 0.11 & 0.05 & 0.02 & 0.01 & 0.00 & 0.00 \\
3 & 0.05 & 0.09 & 0.07 & 0.03 & 0.01 & 0.00 & 0.00 \\
4 & 0.03 & 0.05 & 0.05 & 0.03 & 0.01 & 0.00 & 0.00 \\
5 & 0.01 & 0.02 & 0.02 & 0.02 & 0.01 & 0.00 & 0.00 \\
6 & 0.00 & 0.01 & 0.01 & 0.01 & 0.01 & 0.00 & 0.00 \\
7 & 0.00 & 0.00 & 0.00 & 0.00 & 0.00 & 0.00 & 0.00 \\
\hline \hline
\end{tabular}

Para uma melhor visualização da distribuição preditiva, a mesma foi presentada na forma de gráficos em três dimensões, um diagrama de dispersão( figura 3.11) e um histograma em três dimensões(figura 3.12).

\subsection{Observações sobre o $A B C$}

Após apresentar e discutir o $\boldsymbol{A B C}$, é importante destacar que para o melhor desempenho do método é necessário que o usuário seja capaz de elicitar muito bem a distribuição de probabilidade a priori do estado da natureza, parâmetro do modelo, além de que também é necessário ter um amplo conhecimento em como o experimento em estudo é influenciado por esse parâmetro. Isso já era pré-requisito para a utilização de outros métodos numéricos.

A diferença entre o $\boldsymbol{A} \boldsymbol{B C}$ e outros amostradores está nos casos em que ele pode ser aplicado e em como o $\boldsymbol{A} \boldsymbol{B} \boldsymbol{C}$ reduz a complexidade computacional das ferramentas empregadas. Casos como a abordagem bayesiana para problemas não-paramétricos(abordados no capítulo 4), em que se supõe aleatoriedade das medidas de probabilidades dos eventos que foram observados através de experimentos(impossibilitando uma forma fechada para a função de verossimilhança), ou casos em 
que a utilização de métodos numéricos convencionais é muito custoso são os casos ideais para a utilização do $\boldsymbol{A B C}$.

Outro ponto que precisa ser, e é reforçado pelos algoritmos 11, 5 e 4, é que o $\boldsymbol{A} \boldsymbol{B} \boldsymbol{C}$ não usa a função de verossimilhança. Isto fica claro ao implementar os algoritmos MCMC, que se utilizam da função de verossimilhança do modelo proposto, e ao compará-los com o $\boldsymbol{A B C}$, que incorpora a informação obtida da amostra observada no processo inferencial através do processo de aceitação e rejeição descrito nos algoritmos 1 e 2 . A ideia fundamental do $\boldsymbol{A} B \boldsymbol{C}$ é que se um ponto sorteado aleatoriamente do espaço paramétrico consegue gerar uma amostra que é próxima da amostra que já foi observada, então este ponto do espaço paramétrico deve ser selecionado como uma possível amostra da distribuição posteriori. Não se pode negar que, no amplo sentido da palava, a verossimilhança é utilizada, ela está presente no $\boldsymbol{A B C}$ de forma indireta. 


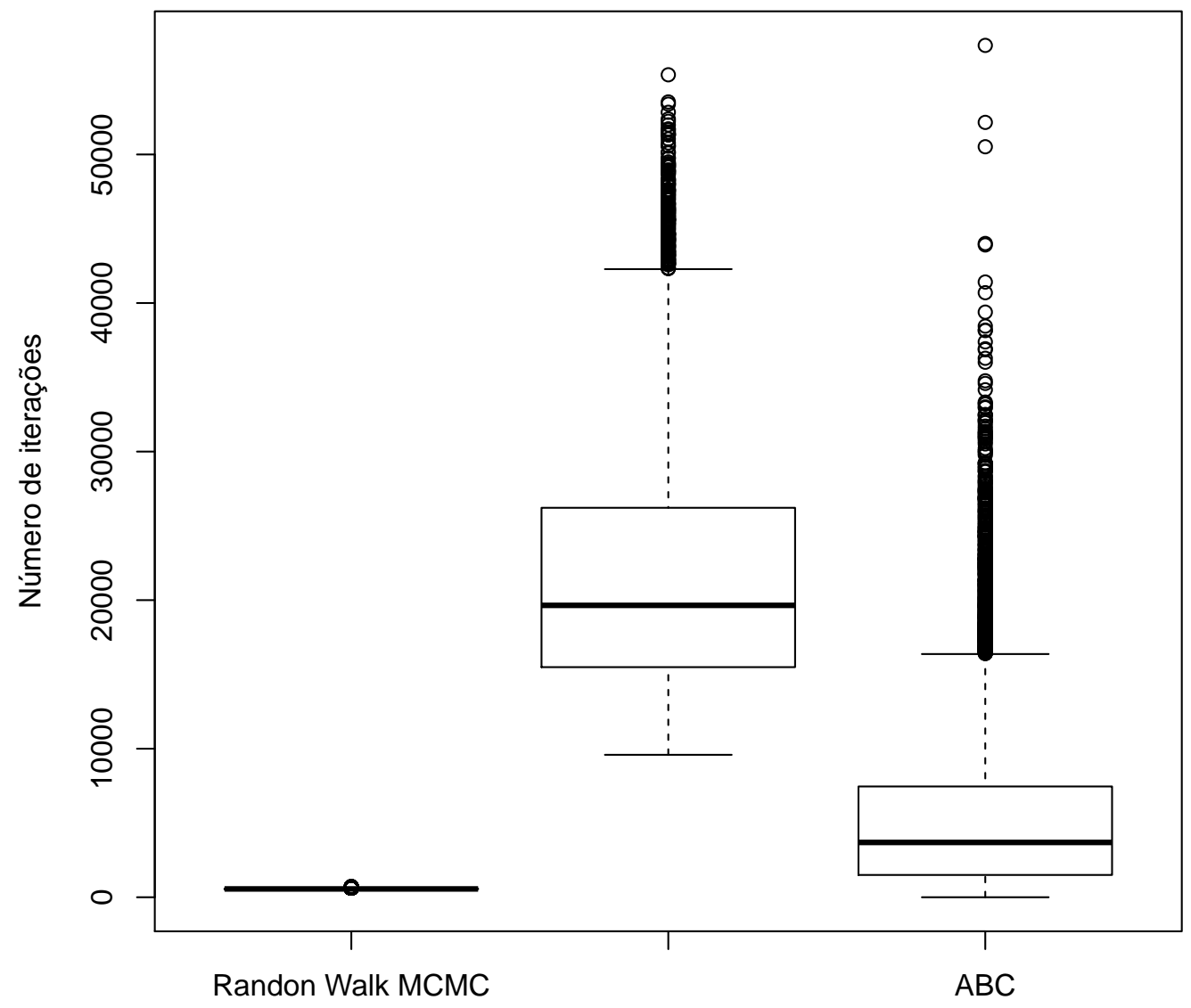

Figura 3.1: Boxplot do número da iterações que cada algoritmo necessitou para gerar a amostra. 

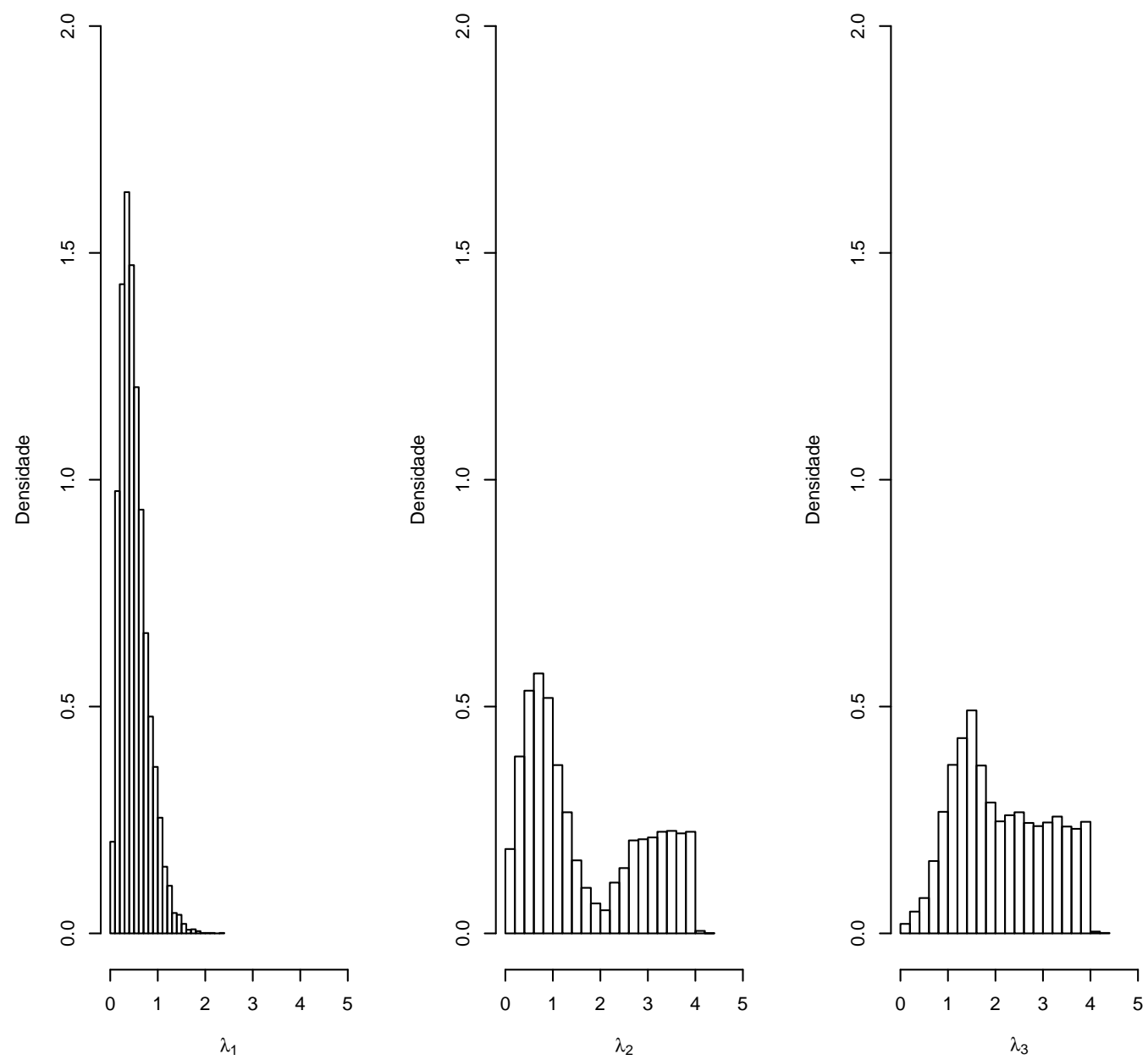

Figura 3.2: Histogramas de observações a posteriori de $\lambda_{1}, \lambda_{2}$ e $\lambda_{3}$ geradas pelo MCMC via Random Walk 

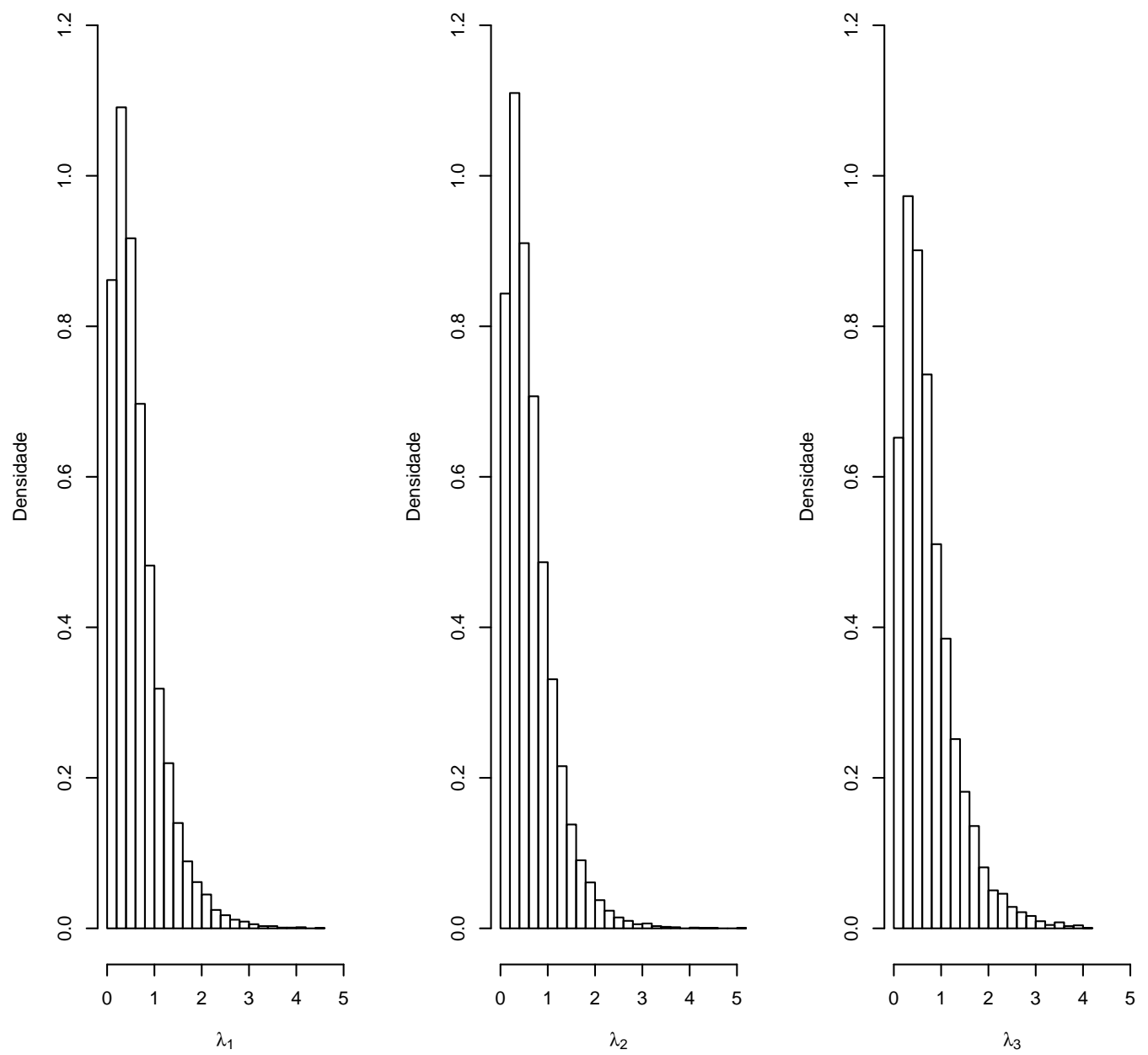

Figura 3.3: Histogramas de observações a posteriori de $\lambda_{1}, \lambda_{2}$ e $\lambda_{3}$ geradas pelo MCMC via Independence Chain 

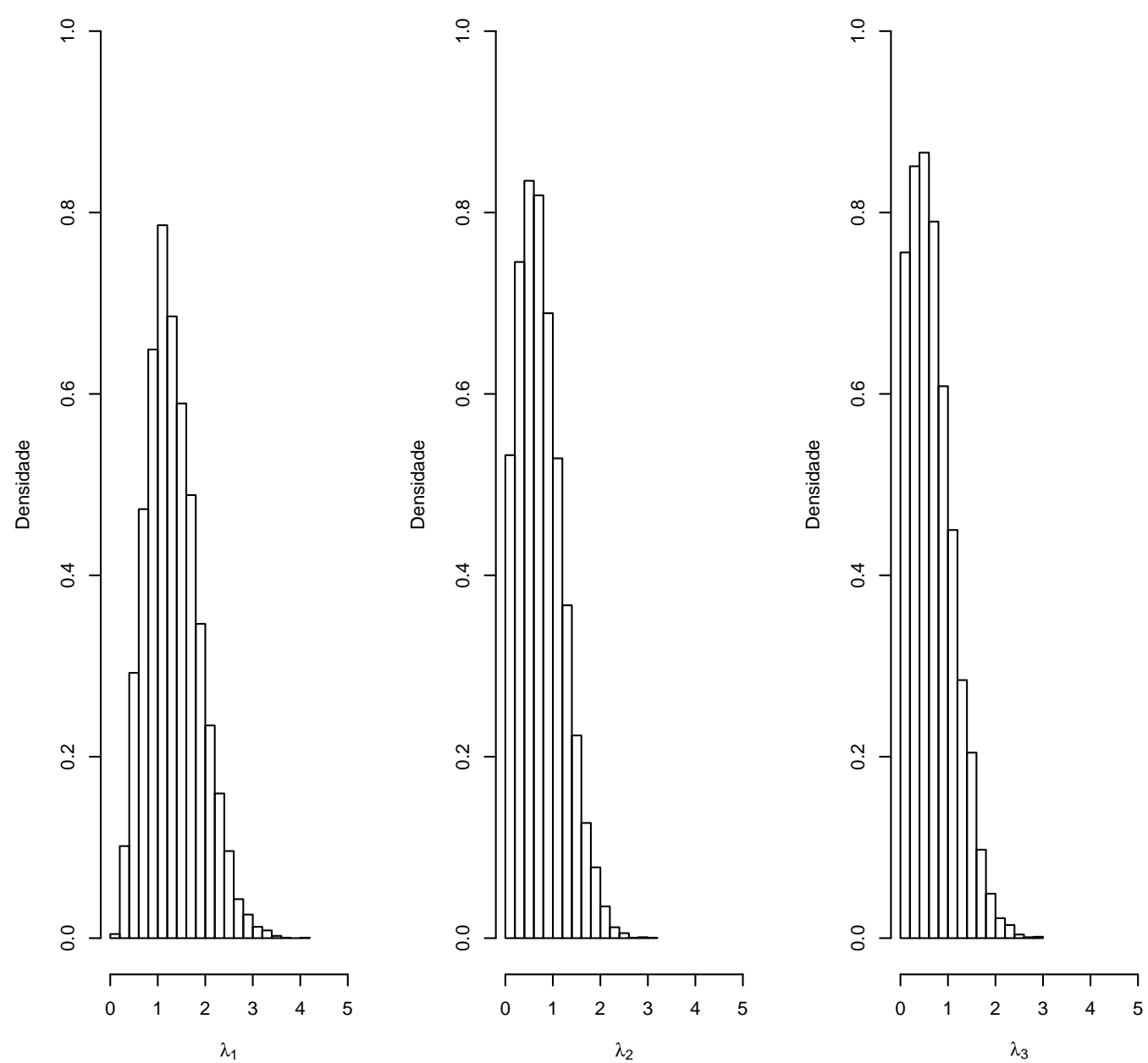

Figura 3.4: Histogramas de observações, geradas pelo $\boldsymbol{A} \boldsymbol{B} \boldsymbol{C}$ a posteriori de $\lambda_{1}, \lambda_{2}$ e $\lambda_{3}$ 


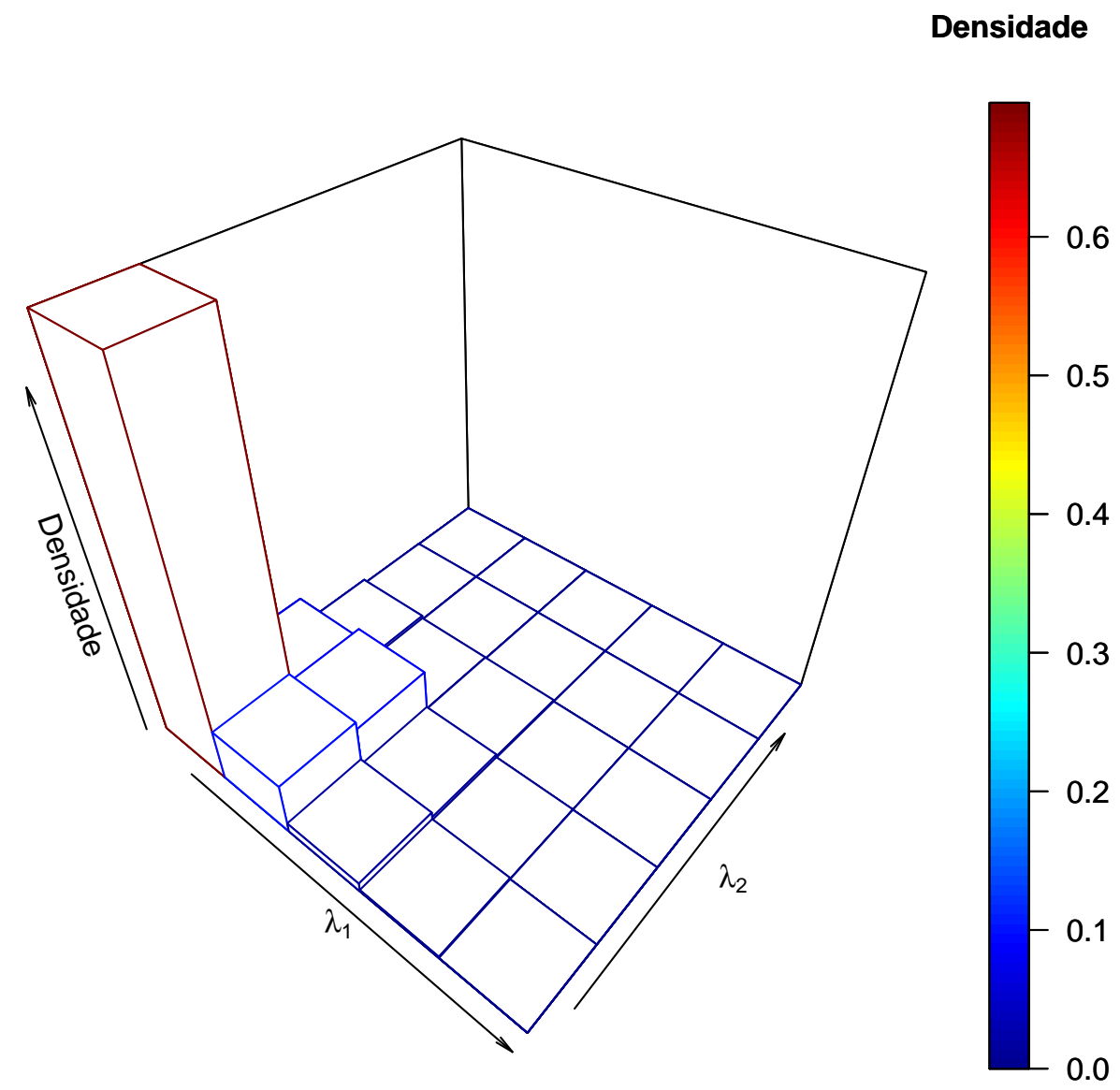

Figura 3.5: Histograma em três dimensões das amostras de $\lambda_{1}$ versus $\lambda_{2}$ geradas pelo Independence Chain $M C M C$ 


\section{Densidade}

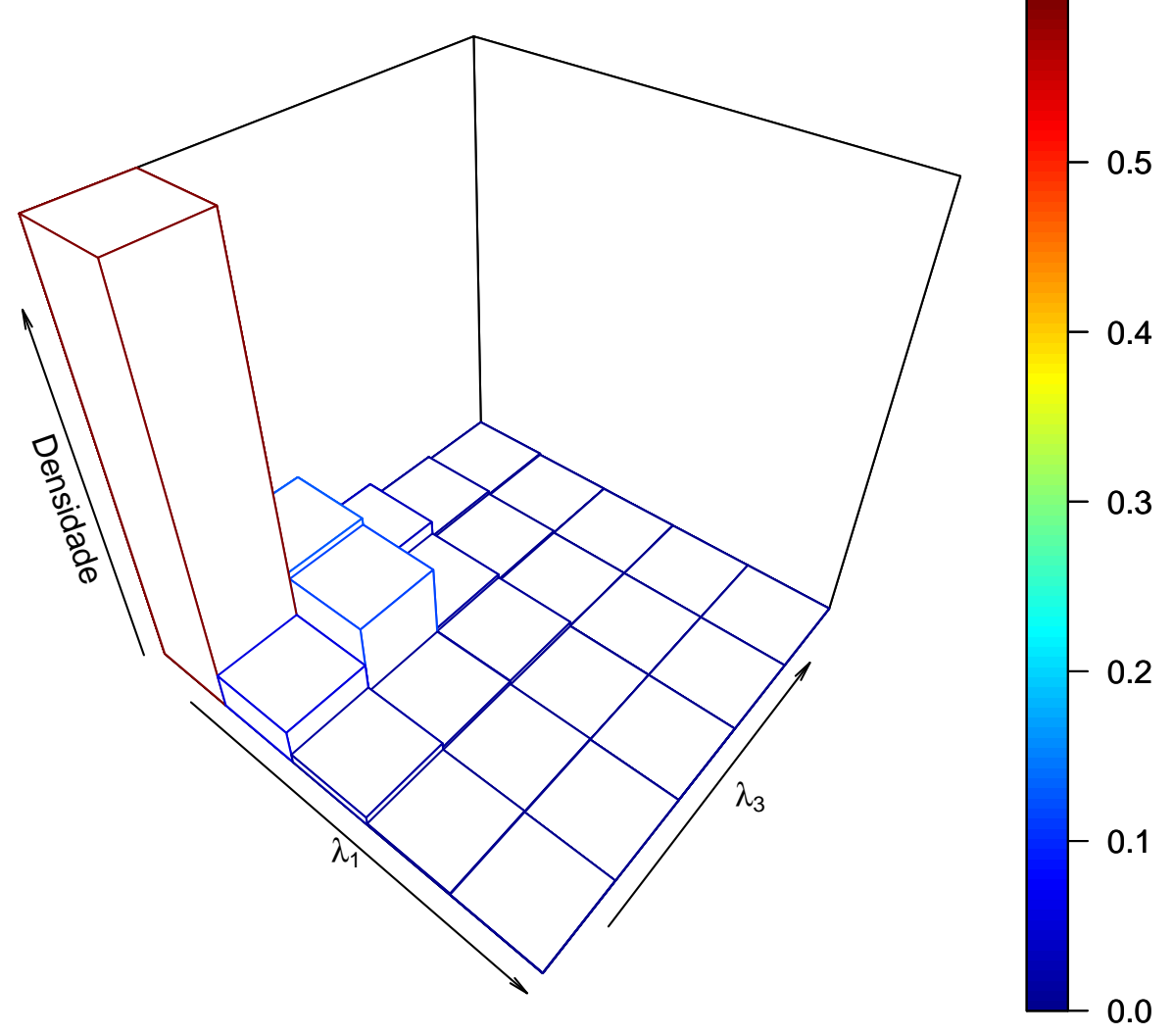

Figura 3.6: Histograma em três dimensões das amostras de $\lambda_{1}$ versus $\lambda_{3}$ geradas pelo Independence Chain $M C M C$ 


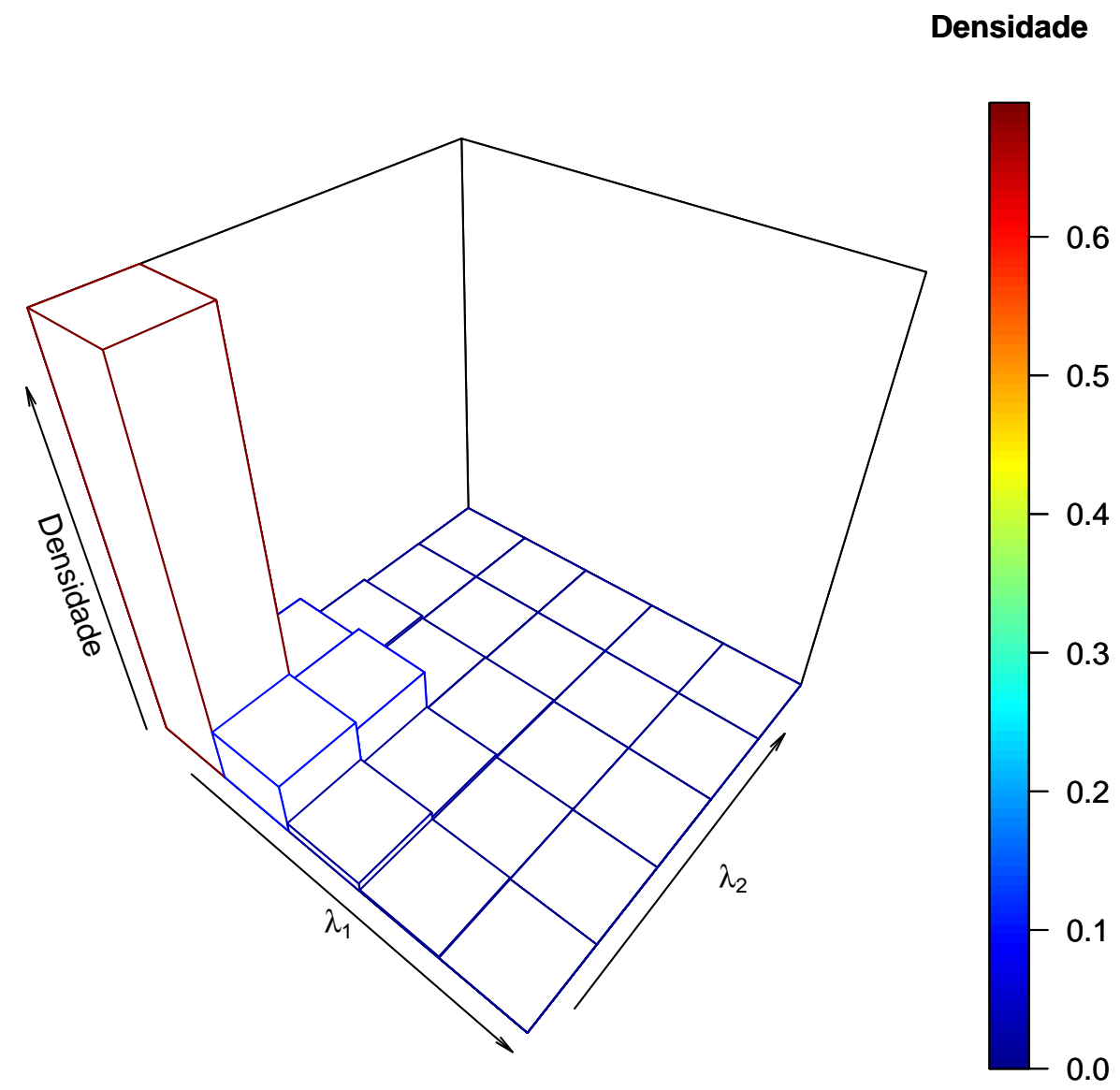

Figura 3.7: Histograma em três dimensões das amostras de $\lambda_{3}$ versus $\lambda_{3}$ geradas pelo Independence Chain $M C M C$ 


\section{Densidade}

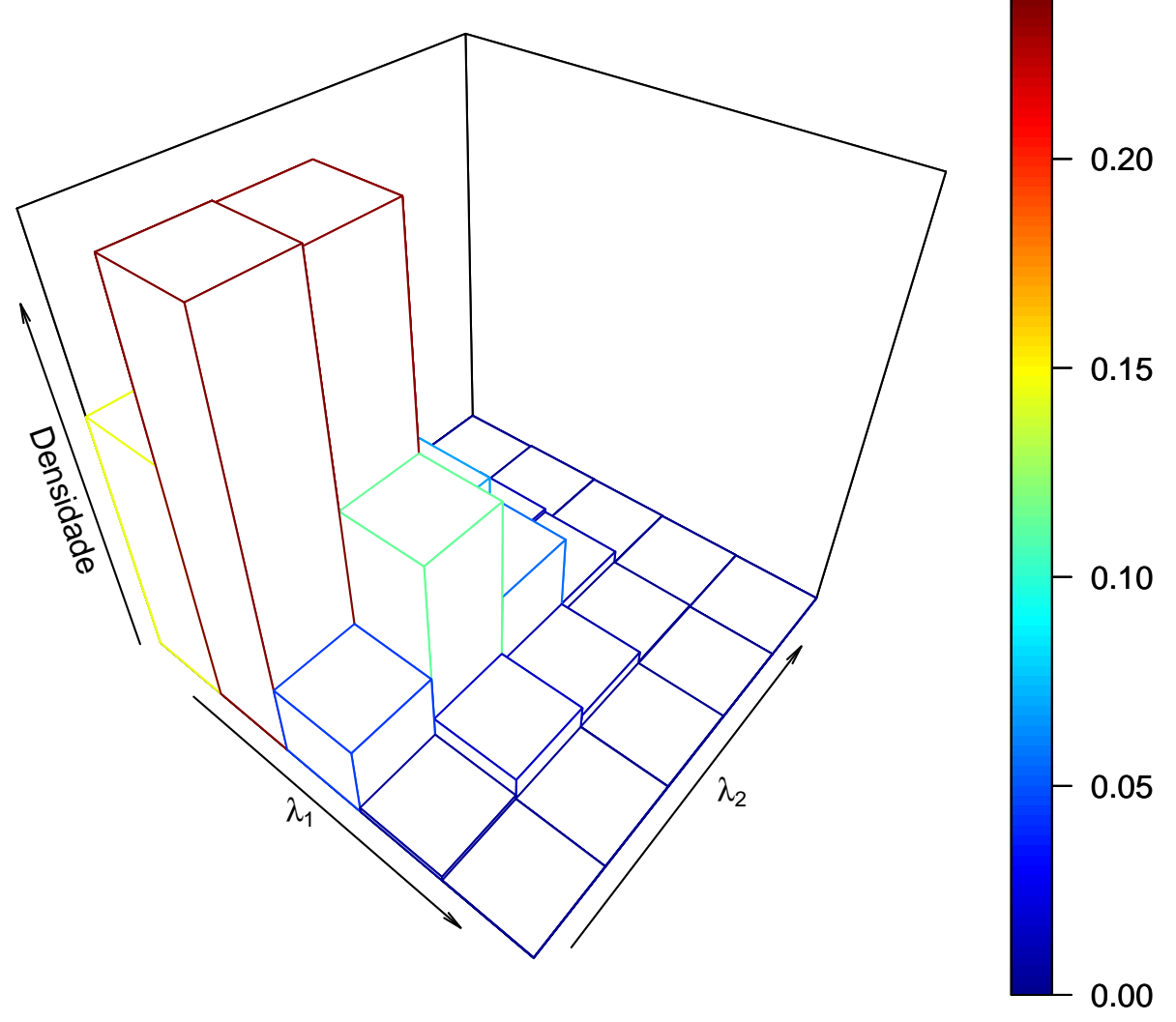

Figura 3.8: Histograma em três dimensões das amostras de $\lambda_{1}$ versus $\lambda_{2}$ geradas pelo $\boldsymbol{A B C}$ 


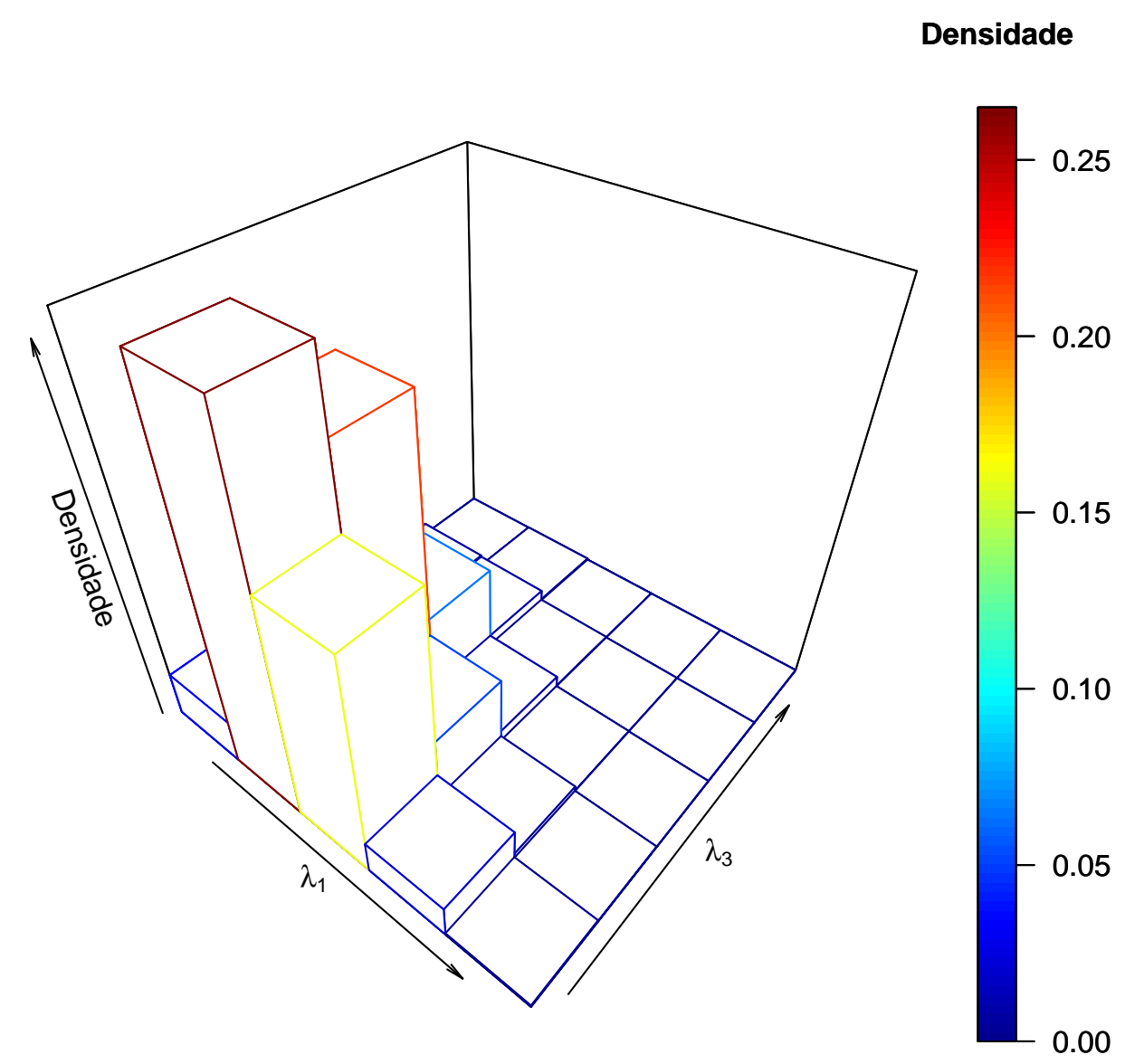

Figura 3.9: Histograma em três dimensões das amostras de $\lambda_{1}$ versus $\lambda_{3}$ geradas pelo $\boldsymbol{A} \boldsymbol{B C}$ 


\section{Densidade}

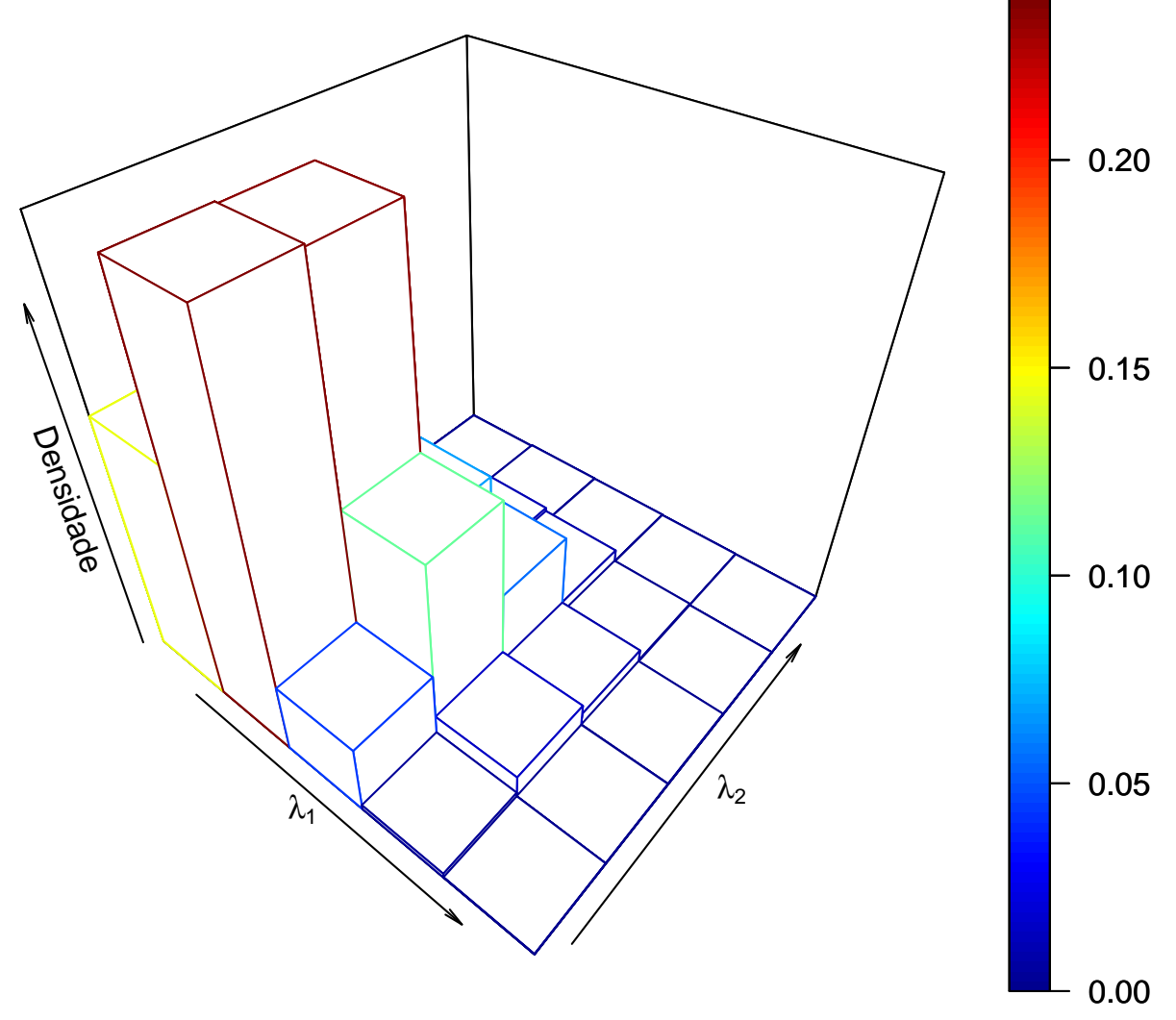

Figura 3.10: Histograma em três dimensões das amostras de $\lambda_{3}$ versus $\lambda_{3}$ geradas pelo $\boldsymbol{A} \boldsymbol{B} \boldsymbol{C}$ 


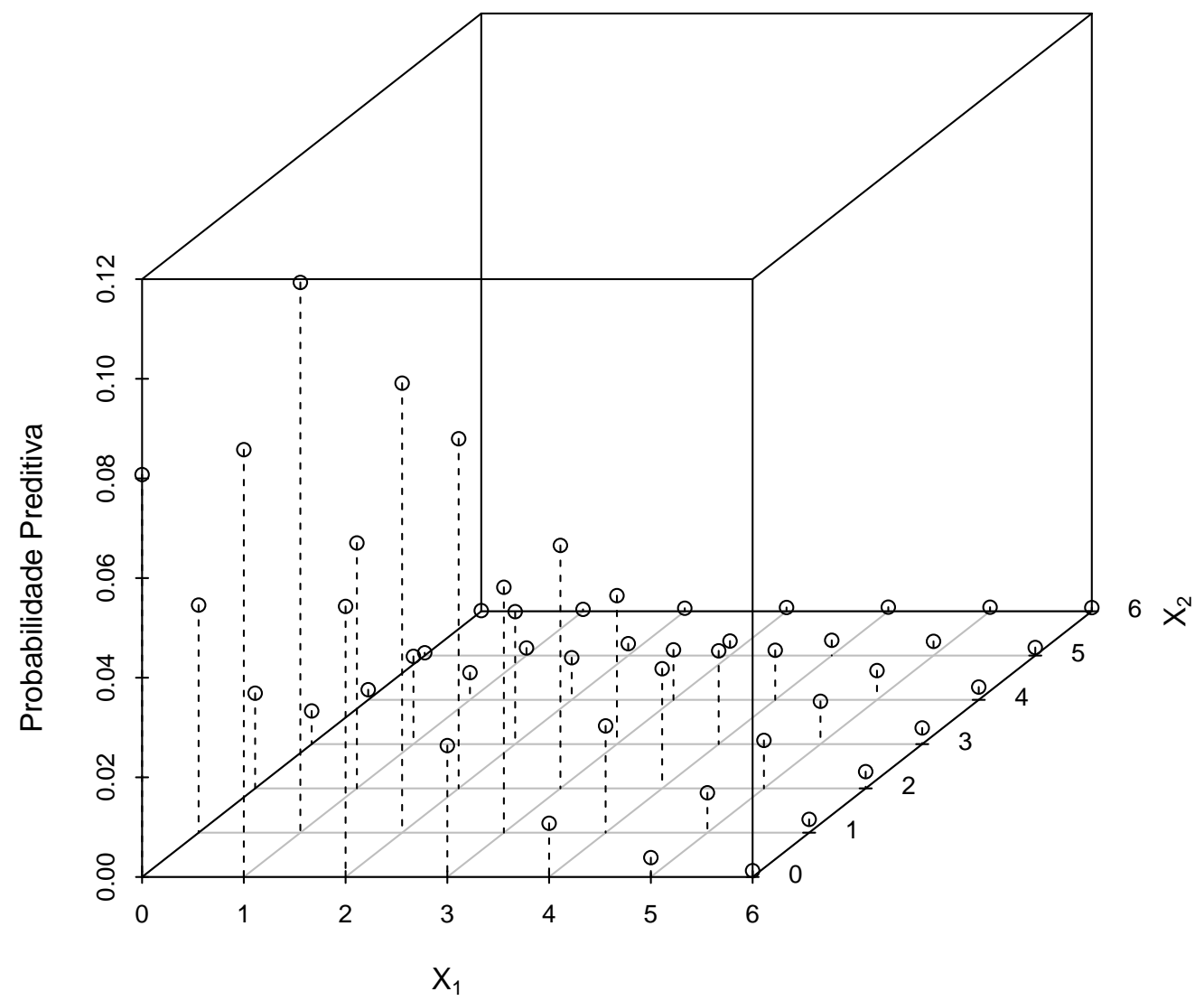

Figura 3.11: Disgrama de dispersão em três dimensões da distribuição preditiva 


\section{Probabilidade}

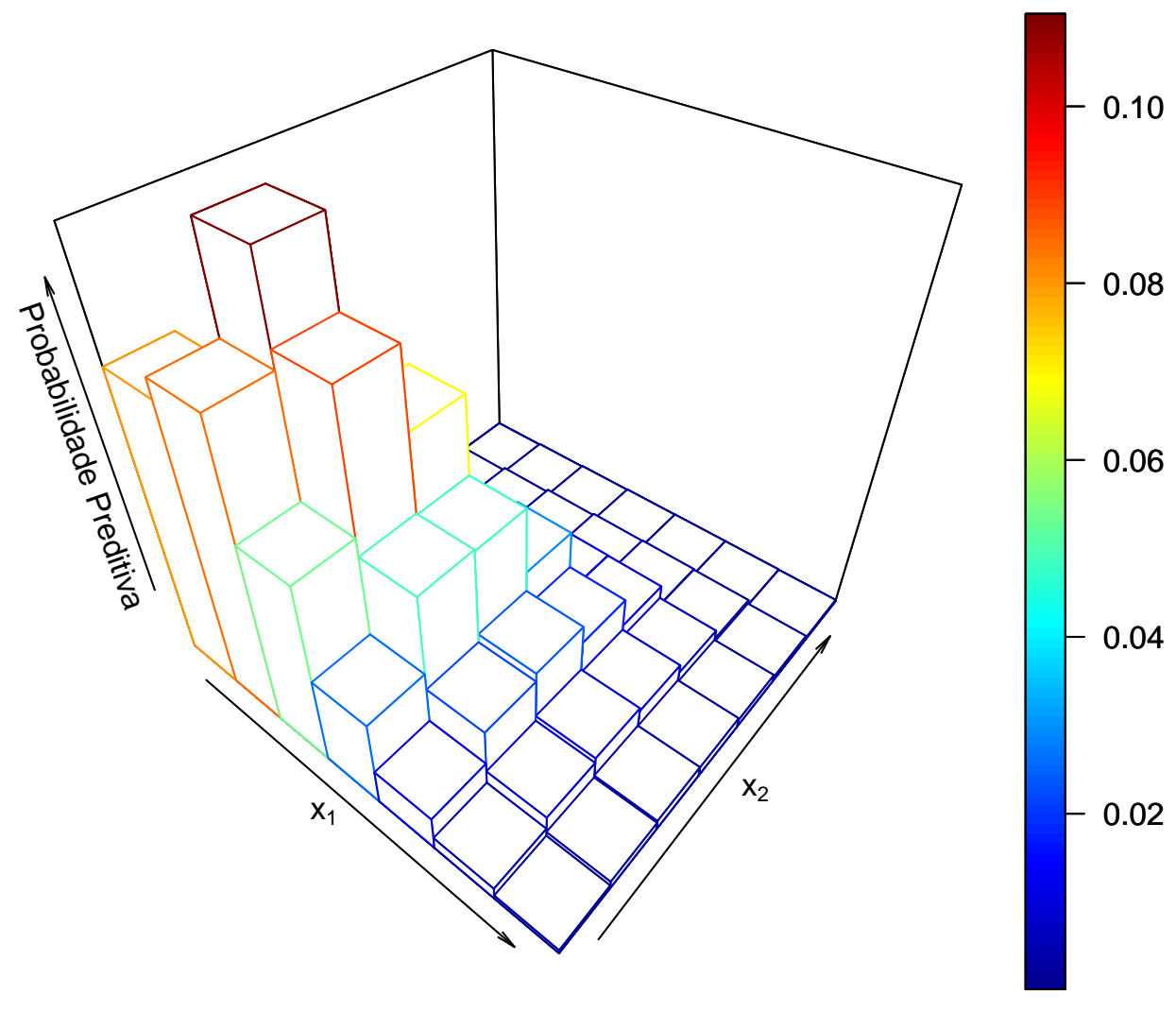

Figura 3.12: Representação da distribuição preditiva na forma de um histograma em três dimensões 


\section{Capítulo 4}

\section{$A B C$ aplicado ao modelo Bayesiano não-paramétrico para o monitoramento "on-line" de qualidade de Taguchi para atributos.}

\subsection{Introdução}

Imagine-se dono de uma fábrica que produz um determinado item. Além dos custos fixos para confeccionar este item existem os extras que incidem sobre o mesmo quando um item não-conforme é produzido. Por exemplo, quando uma montadora de carros detecta que um lote foi vendido com uma não-conformidade em alguns dos itens que compõem o veículo é obrigação da montadora fazer um recall para corrigir a não-conformidade. Esse processo gera custos extras para a montadora. Portanto, é necessário ter um procedimento para o controle da qualidade da linha de produção.

Taguchi et al. (1989) apresentam um procedimento de monitoramento "on-line" para atributos, baseado em fatores econômicos (custos de produção e de inspeção de itens, dentre outros). O objetivo de tal procedimento foi minimizar custos associados a um processo de produção sujeito a deterioração.

Em resumo, Taguchi et al. (1989) examinaram um processo de produção que manufaturava um único item por vez. A fração de itens não-conformes do processo, em um momento desconhecido (aleatório) passa de um valor inicial zero (estado de processo sob controle) para um valor positivo $\theta$ (estado de processo fora de controle). O procedimento de monitoramento consiste em inspecionar o último item de cada $m$ itens produzidos. Assim que um item não conforme é verificado, o processo é interrompido e ajustado para que volte à condição inicial, fechando um ciclo de produção (período entre um (re)começo do processo de produção até a próxima interrupção para ajuste). A determinação do intervalo de inspeção ótimo, $m^{*}$, é então realizada de modo a minimizar o custo esperado a longo prazo do procedimento, por unidade manufaturada. Taguchi et al. (1989) derivaram uma expressão matemática para $m^{*}$ em duas situações, caso 1 (em que $\theta=1$ e o processo passa a produzir somente peças não-conformes) e caso 2 (que é quando $0<\theta<1$ e o processo ainda consegue produzir peças conforme as especificações) embora não especifique um mecanismo de falha do processo (a distribuição de probabilidade do tempo de espera para que o processo saia do controle) ou um modelo estatístico para a observação dos itens produzidos, cujos parâmetros devem ser estimados a fim de determinar $m^{*}$.

Tsunemi et al. (2012) apresentam um modelo Bayesiano não-paramétrico para o procedimento de Taguchi para o monitoramento "on-line" de qualidade para atributos com o objetivo de modelar a gradual deterioração do processo de produção durante um ciclo de produção (um outro caso diferente dos casos 1 e 2 citados anteriormente) e, além disso, incorporar a opinião de um especialista no processo de produção.

Ainda em Tsunemi et al. (2012) é argumentado que a deterioração contínua da qualidade não deve ser negligenciada quando a estratégia de monitoramento de Taguchi é utilizada. A sequência crescente da fração de itens não-conformes durante um ciclo de produção é vista como uma função de distribuição discreta. Além disso, também é argumentado que o modelo em questão pode ser aplicado a uma ampla gama de processos de produção devido à sua estrutura não-paramétrica menos restritiva. 


\subsection{O modelo}

A seguir, o modelo proposto em Tsunemi et al. (2012), será exposto e discutido, começando pelas suposições.

\subsubsection{Suposições}

Tsunemi et al. (2012) consideram uma medida $\mathcal{P}: \mathcal{B}\left(\overline{\mathbb{R}}_{+}^{*}\right) \rightarrow \mathbb{R}_{+}$finita na $\sigma$-álgebra de Borel na reta estendida $\left(\overline{\mathbb{R}}_{+}^{*}=\mathbb{R}_{+}^{*} \cup\{\infty\}=(0, \infty]\right)$ tal que $\mathcal{P}\left(\overline{\mathbb{R}}_{+}^{*}\right)=1$. Também considera-se $F$ como sendo a distribuição de probabilidade definida em $\mathbb{R}_{+}^{*}$ associada com a medida $\mathcal{P}$ (para cada $t \in \mathbb{R}_{+}^{*}$, $F(t)=\mathcal{P}((0, t]))$.

Seja $\left(X_{n}\right)_{n \geq 1}$ um processo estocástico tal que $X_{n}=1$, se o n-ésimo item produzido em um ciclo de produção é um item não-conforme, e $X_{n}=0$, caso contrário. Supõe-se que quando o nésimo item está para ser produzido, a fração de itens não-conformes no processo (não observada) é $\mathcal{P}((0, n])=F(n), n \geq 1$. Então, $(F(n))_{n \geq 1}$ representa uma sequência de frações de itens nãoconformes ao longo de um ciclo de produção. Seja $\eta$ o tempo de espera até que o processo comece a sair de controle (tempo este, aleatório e não observável), $\eta=\inf \{n \leq 1: F(n)>0\}$.

Supõe-se também que, para cada $n \geq 1$, as variáveis aleatórias $X_{1}, \ldots, X_{n}$ são condicionalmente independentes dados $\mathcal{P}((0,1]), \ldots, \mathcal{P}((0, n])$ e $\eta$. Fixado $n \in \mathbb{N}^{*}$, supõe-se também que, para $i=1, \ldots, n$

$$
\begin{aligned}
P\left(X_{i}\right. & =1 \mid \mathcal{P}((0,1]), \mathcal{P}((1,2]), \ldots, \mathcal{P}((n-1, n]), \eta)=P\left(X_{i}=1 \mid \mathcal{P}((0,1]), \mathcal{P}((1,2]), \ldots, \mathcal{P}((i-1, i])\right) \\
& =\mathcal{P}((0,1])+\mathcal{P}((1,2])+\ldots+\mathcal{P}((i-1, i])=\mathcal{P}((0, i])=p_{i} .
\end{aligned}
$$

Considerando todas essas suposições feitas, pode-se dizer que $\left(X_{n}\right)_{n>1}$ são, dadas $\mathcal{P}$ e $\eta$, variáveis, condicionalmente, independentes, não identicamente distribuídas Bernoulli com parâmetros correspondentes $(F(n))_{n \geq 1}$.

De acordo com a estratégia de Taguchi para controle de qualidade, observa-se o número de inspeções até ser verificado o primeiro item não-conforme. Seja $\tau=\inf \left\{k \geq 1: X_{k m}=1\right\}$ a variável aleatória que representa esse número de inspeções, a distribuição amostral de $\tau$ é da forma,

$$
\begin{aligned}
P(\tau & =k \mid \mathcal{P}((0,1]), \ldots, \mathcal{P}((k m-1, k m]), \eta)=P\left(\bigcap_{i=1}^{k-1}\left\{X_{i m}=0\right\} \cap\left\{X_{[k m]}\right\} \mid \mathcal{P}((0,1]), \ldots, \mathcal{P}((k m-1, k m]), \eta\right) \\
& =\left\{\prod_{i=1}^{k-1} P\left(X_{i m}=0 \mid \mathcal{P}((0,1]), \ldots, \mathcal{P}((i m-1, i m])\right)\right\} \times P\left(X_{k m}=1 \mid \mathcal{P}((0,1]), \ldots, \mathcal{P}((k m-1, k m])\right) \\
& =\left\{\prod_{i=1}^{k-1}\left(1-P_{i m}\right)\right\} P_{k m}=\left\{\prod_{i=1}^{k-1}\left(\sum_{j=i+1}^{k+1} \mathcal{P}\left(D_{j}\right)\right)\right\} \times\left\{\sum_{j=1}^{k} \mathcal{P}\left(D_{j}\right)\right\},
\end{aligned}
$$

onde, $D_{j}=((j-1) m, j m], i=1, \ldots, k$ e $D_{k+1}=(k m, \infty]$.

É considerado que $\mathcal{P}$, dado $\eta$, é distribuído de acordo com um processo de Dirichlet com medida suporte finita $\mu_{\eta}: \mathcal{B}\left(\overline{\mathbb{R}}_{+}^{*}\right) \rightarrow \mathbb{R}_{+}$. A distribuição de $\eta$ é dada da forma de um modelo hierárquico; seja $\rho \sim \operatorname{Beta}(a, b), \eta \mid \rho$ segue uma distribuição geométrica com média $\rho^{-1}$. Marginalmente obtêm-se,

$$
P(\eta=t)=\frac{\Gamma(a+b) \Gamma(a+1) \Gamma(b+t)}{\Gamma(a) \Gamma(b) \Gamma(a+b+t+1)}
$$

Aqui $\rho$ é usado apenas como um artificio matemático para dar a $\eta$ a distribuição desejada por Tsunemi et al. (2012).

\subsubsection{Modelo}

Com a informação $\tau=k$, observação do primeiro item não-conforme na $k$-ésima inspeção, a distribuição de $\mathcal{P} \mid \eta, \tau=k$ será derivada, através dos resultados descritos em Tsunemi et al. (2012).

Teorema 4.2.1. Seja $\mathcal{P}, \eta$ e $\tau$ definidos como na seção 4.2.1 e $\left(B_{1}, \ldots, B_{n}\right)$ uma partição de $\overline{\mathbb{R}}_{+}^{*}$, e para $k \leq 1$, considere a partição $\left(D_{1}, \ldots, D_{k+1}\right)$, onde, $D_{j}=((j-1) m, j m], i=1, \ldots, k e$ 
$D_{k+1}=(k m, \infty]$. Então

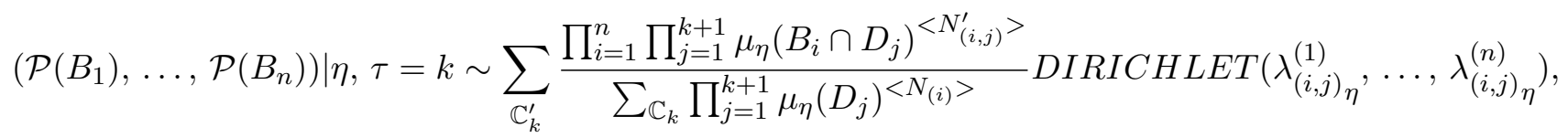

onde, $\mathbb{C}_{k}=\left\{\left(j_{1}, \ldots, j_{k}\right): j_{l} \in\{l+1, \ldots, k+1\}, l=1, \ldots, k-1\right.$ e $\left.j_{k} \in\{1, \ldots, k\}\right\}, \mathbb{C}_{k}^{\prime}=$ $\left\{\left(\left(i_{1}, j_{1}\right), \ldots,\left(i_{k}, j_{k}\right)\right): i_{l} \in\{i, \ldots, n\} \forall l=1, \ldots, k, l j_{l} \in l+1, \ldots, k+1, l=1, \ldots, k-1\right.$ e $j_{k} \in$ $\{1, \ldots, k\}\}, N_{(j)}=\sum_{l=1}^{K} I_{\{j\}}\left(j_{l}\right), N_{(i, j)}^{\prime}=\sum_{l=1}^{K} I_{(i, j)}\left(i_{l}, j_{l}\right), u^{<k>}=\prod_{j=0}^{k-1}(u+j)$ para $u>0 e$ $k \geq 1$ e $\lambda_{(i, j)}^{(i)}=\mu_{\eta}\left(B_{i}\right)+\sum_{l=1}^{K} I_{\{j\}}\left(j_{l}\right)$.

Corolário 4.2.1. Sob as condições do Teorema 4.2.1, seja $t \in \mathcal{R}_{+}^{*}$ e considere $n=2, B_{1}=(0, t]$ e $B_{2}=(t, \infty]$. Então

$$
F(t) \mid \eta, \tau=k \sim \sum_{s=0}^{k} p_{\eta}^{(s)} \times \operatorname{Beta}\left(\mu_{\eta}((0, t])+s, \mu_{\eta}((t, \infty])+k-s\right),
$$

onde

$$
p_{\eta}^{(s)}=\frac{\sum_{\mathbb{C}_{k, s}^{\prime}} \prod_{j=1}^{k+1} \mu_{\eta}\left((0, t] \cap D_{j}\right)^{<N_{(1, j)}^{\prime}>} \mu_{\eta}\left(D_{j} \cap(t, \infty]\right)^{<N_{(2, j)}^{\prime}>}}{\sum_{\mathbb{C}_{k}} \prod_{j=1}^{k+1} \mu_{\eta}\left(D_{j}\right)^{<N_{(i)}>}},
$$

com $s=\sum_{l=1}^{k} I_{\{1\}}\left(i_{l}\right), \mathbb{C}_{k, s}^{\prime}=\left\{\left(i_{1}, j_{1}\right), \ldots,\left(i_{k}, j_{k}\right) \in \mathbb{C}_{k}^{\prime}: \sum_{l=1}^{k} I_{\{1\}}\left(i_{l}\right)=s\right\}$.

O Teorema 4.2.1 apresenta a distribuição a posteriori exata do vetor $\left(\mathcal{P}\left(B_{1}\right), \ldots, \mathcal{P}\left(B_{n}\right)\right) \mid \eta, \tau=$ $k, \forall n$, e $\forall \mathcal{P}\left(B_{1}\right), \ldots, \mathcal{P}\left(B_{n}\right), \ldots$, quando $n=2$ e $B_{1}$ e $B_{2}$ nas condições do Colorário 4.2.1, tem-se, como caso particular, a distribuição exata a posteriori de $F(t) \mid \eta, \tau=k$.

Como é desejado estimar a proporção de itens não-conformes ao longo do processo, é necessário de um estimador para $F(t), \forall t \in \mathbb{N}$.

Corolário 4.2.2. Sob as condições do Teorema 4.2.1, a estimativa de Bayes para $F(t), t \in \mathbb{N}^{*}$, com respeito a perda quadrática quando $\tau=k$ é dada por

$$
E[F(t) \mid \tau=k]=\sum_{j=1}^{\infty}\left\{\frac{\Gamma(b+j-1)}{\Gamma(a+b+j)}\left[\sum_{s=0}^{k} p_{j}^{(s)} \frac{\mu_{j}((0, t]+s)}{\mu_{j}\left(\overline{\mathbb{R}}_{+}^{*}\right)+k}\right]\right\} .
$$

Como um sub-produto desse modelo, tem-se também, a distribuição exata do momento em que o processo de produção sai de controle, $\eta$.

Corolário 4.2.3. Sob as condições do Teorema 4.2.1, a distribuição condicional, exata, de $\eta \mid \tau=k$, é dada por

$$
P(\eta=i \mid \tau=k)=\frac{\sum_{\mathbb{C}_{k}} \frac{\prod_{j=1}^{k+1} \mu_{i}\left(D_{j}\right)^{<N_{(j)}>}}{\mu_{i}\left(\mathbb{\mathbb { R }}_{+}^{*}\right)^{<k>}} \frac{\Gamma(b+i-1)}{\Gamma(a+b+i)}}{\sum_{l=1}^{\infty}\left\{\sum_{\mathbb{C}_{k}} \frac{\prod_{j=1}^{k+1} \mu_{l}\left(D_{j}\right)^{<N}(j)>}{\mu_{l}\left(\overline{\mathbb{R}}_{+}^{*}\right)^{<k>}} \frac{\Gamma(b+l-1)}{\Gamma(a+b+l)}\right\}} .
$$

Todas as demonstrações do resultados aqui apresentados estão em Tsunemi et al. (2012, Apêndice A).

\subsubsection{Vantagens e desvantagens}

No campo das ideias, teorias e modelos, Tsunemi et al. (2012) introduz um modelo inovador e se utiliza de uma teoria sofisticada, possibilitando assim dispensar aproximações probabilísticas, mas tudo isso tem um preço. A alta complexidade computacional é a principal desvantagem.

O calculo dos pesos de cada parcela das misturas de Dirichlet (Betas, no caso $n=2$ ) é onde se encontra toda a complexidade computacional do problema. No termo $p_{\eta}^{(s)}$ os conjuntos $\mathbb{C}_{k}, \mathbb{C}_{k}^{\prime}$ e 
$\mathbb{C}_{k, s}^{\prime}$ são usados como conjuntos de índices dos somatórios, onde cada elemento de cada um desses conjuntos será utilizado na função $\left\langle N_{(i, j)}^{\prime}>\right.$ e $\left\langle N_{(j)}>\right.$, onde $(i, j)$ é parte de um elemento de $\mathbb{C}_{k, s}^{\prime}$ (ou $\mathbb{C}_{k}^{\prime}$ ) e $(j)$ é uma parte de um elemento de $\mathbb{C}_{k}$.

Do ponto de vista computacional, $\mathbb{C}_{k}$ e $\mathbb{C}_{k}^{\prime}$ são matrizes, e cada linha dessas matrizes é o índice usado no somatório. Usando o Teorema 4.2.1 como base, pode-se dizer que $\mathbb{C}_{k}$ é uma matriz de $k$ colunas e $k \times k$ ! linhas. Já $\mathbb{C}_{k}^{\prime}$ é uma matriz de $2 \times k$ colunas e $n^{k} \times k \times k$ ! linhas. Quando $n=2$, temos que $\mathbb{C}_{k}^{\prime}$ tem $2^{k} \times k \times k$ ! linhas. Notem que quanto maior o número de linhas em $\mathbb{C}_{k}^{\prime}$, maior é o número de Dirichlet's na mistura de distribuições e por consequência o número de pesos $p_{j}^{(s)}$ a serem calculados na equação 4.6. Em síntese, quanto maior $k$, maior é a complexidade computacional do problema.

Nas figuras 4.1 e 4.2 , vemos como os números de linhas das matrizes $\mathbb{C}_{k}^{\prime}$ e $\mathbb{C}_{k}$ crescem de acordo com $k$. Nota-se um crescimento de forma exponencial. Veja que se $k=8$ e $n=2$ a matriz $\mathbb{C}_{k}^{\prime}$ será uma matriz de dimensão $16 \times 82575360$, totalizando 1321205760 elementos e, de acordo com o programa $\boldsymbol{R}$, são necessários $9,8 G B$ de memória ram para poder armazenar e manipular os elementos da matriz $\mathbb{C}_{k}^{\prime}$. Assim, constata-se que é de fundamental importância uma alternativa computacional que possa ser usada de forma prática e, comercial em certo nível. Por exemplo o problema da politica de recall utilizada por montadoras de carros, abordado em Tsunemi et al. (2012).

\section{3 $A B C$ aplicado ao modelo não paramétrico}

Uma alternativa à formula direta proposta em Tsunemi et al. (2012) seria a geração de amostras da distribuição de $F(t) \mid \tau=k$ e então calcular, aproximadamente, $E[F(t) \mid \tau=k], \forall t$, através da média amostral das observações geradas. Esta tarefa é tão hercúlea quanto calcular diretamente $E[F(t) \mid \tau=k]$ pela equação 4.6 .

A escolha do $\boldsymbol{A B C}$ parece ideal para gerar amostras da mistura de processos de Dirichilet's descrita no teorema 4.2.1. Por não se utilizar da função de verossimilhança, espera-se que o $\boldsymbol{A} \boldsymbol{B} \boldsymbol{C}$ não sofra das mesmas complexidades computacionais que a distribuição exata sofre. Tamém esperase que o $\boldsymbol{A B C}$ consiga gerar amostras da distribuição 4.4 para valores maiores $\tau \geq 8$.

Para implementar um $\boldsymbol{A} \boldsymbol{B} \boldsymbol{C}$ que irá gerar amostras da distribuição 4.4, é necessário estipular condições especificas, por exemplo, qual versão do $\boldsymbol{A} B \boldsymbol{C}$ usar, como gerar observações da distribuição a priori, e como replicar o experimento observado.

O $\boldsymbol{A} \boldsymbol{B} \boldsymbol{C}$-Rejeição para variáveis discretas será o utilizado para gerar observações da mistura de processos de Dirichlet's em questão.

Para gerar observações da distribuição a priori, é necessário saber como gerar amostras de um processo de Dirichlet, discutido na subseção 4.3.1. A descrição de como replicar o experimento observado, inspeções na linha de produção, está presente na subseção 4.3.2. E por fim, o algoritmo $\boldsymbol{A} \boldsymbol{B} \boldsymbol{C}$ completo para gerar amostras de $\left(\mathcal{P}\left(B_{1}\right), \ldots, \mathcal{P}\left(B_{n}\right)\right) \mid \eta, \tau=k$ será construido na subseção 4.3 .3

\subsubsection{Gerando observações de um processo de Dirichlet}

O primeiro ponto importante que precisa ser exposto na construção de um algoritmo $\boldsymbol{A} \boldsymbol{B} \boldsymbol{C}$ é a geração de observações da distribuição a priori. Tsunemi et al. (2012) determinam o processo de Dirichlet (definido em Ferguson (1973)) como distribuição a priori do modelo apresentado no teorema 4.2.1. Existem algumas representações do processo de Dirichlet conhecidas na literatura, como o stickbreaker (veja Sethuraman (1994)), a mais conhecida.

Um outro método, desenvolvido mais recentemente, promete ser mais eficiente na geração de observações de um processo de Dirichlet que o stickbreaker. Zarepour e Labadi (2011) mostram várias aproximações do processo de Dirichlet, como a própria representação de Ferguson (1973), Sethuraman (1994), dentre outros, e apresentam as dificuldades de cada uma delas. A alternativa apresentada, baseada no processo gama, é chamada de Rapid Simulation of a Dirichlet Process.

O método de Zarepour e Labadi (2011) para gerar observações de um processo de Dirichlet com uma medida suporte $\alpha H$ consiste em: 

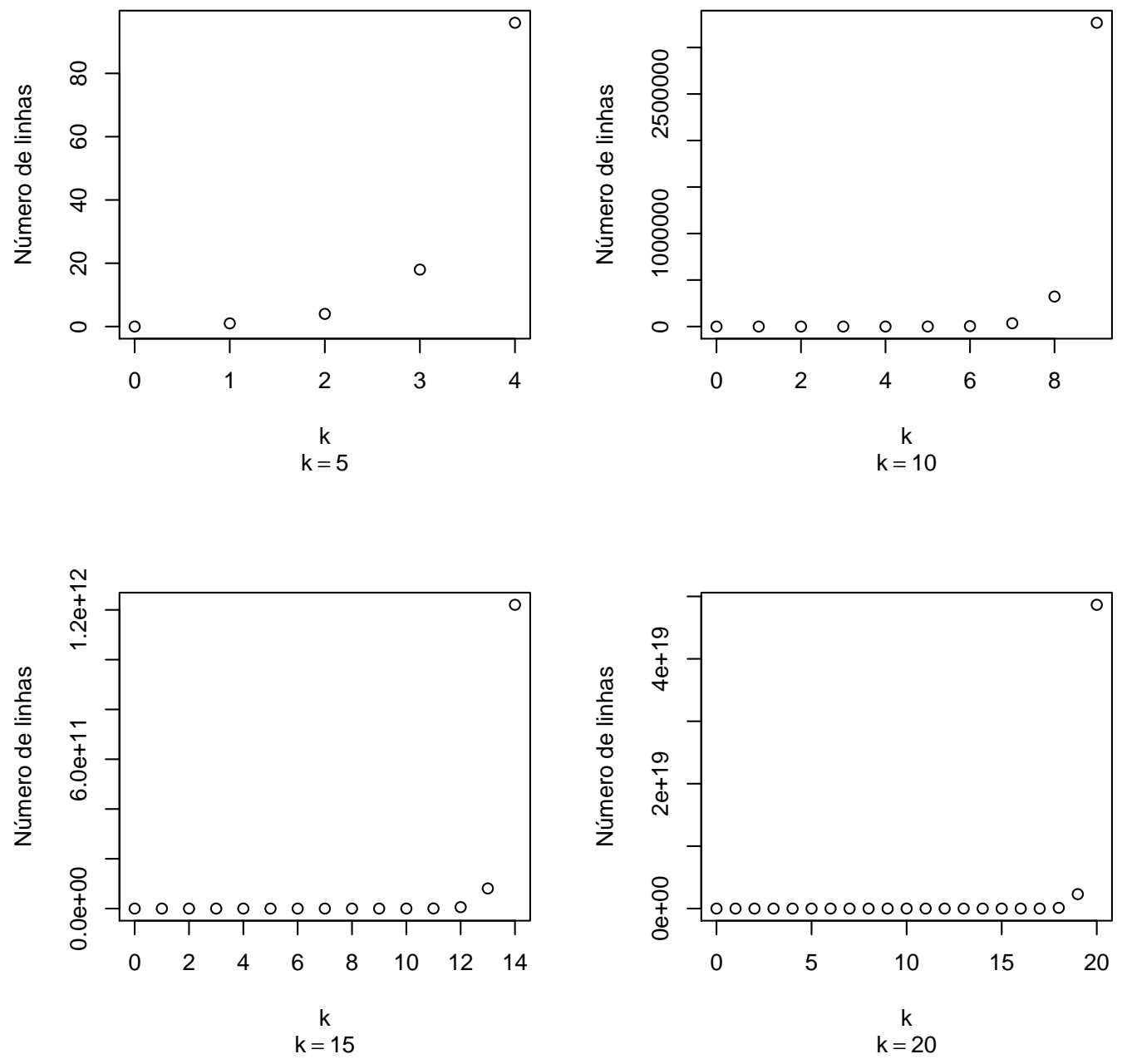

Figura 4.1: Número de linhas da matriz $\mathbb{C}_{k}$ 

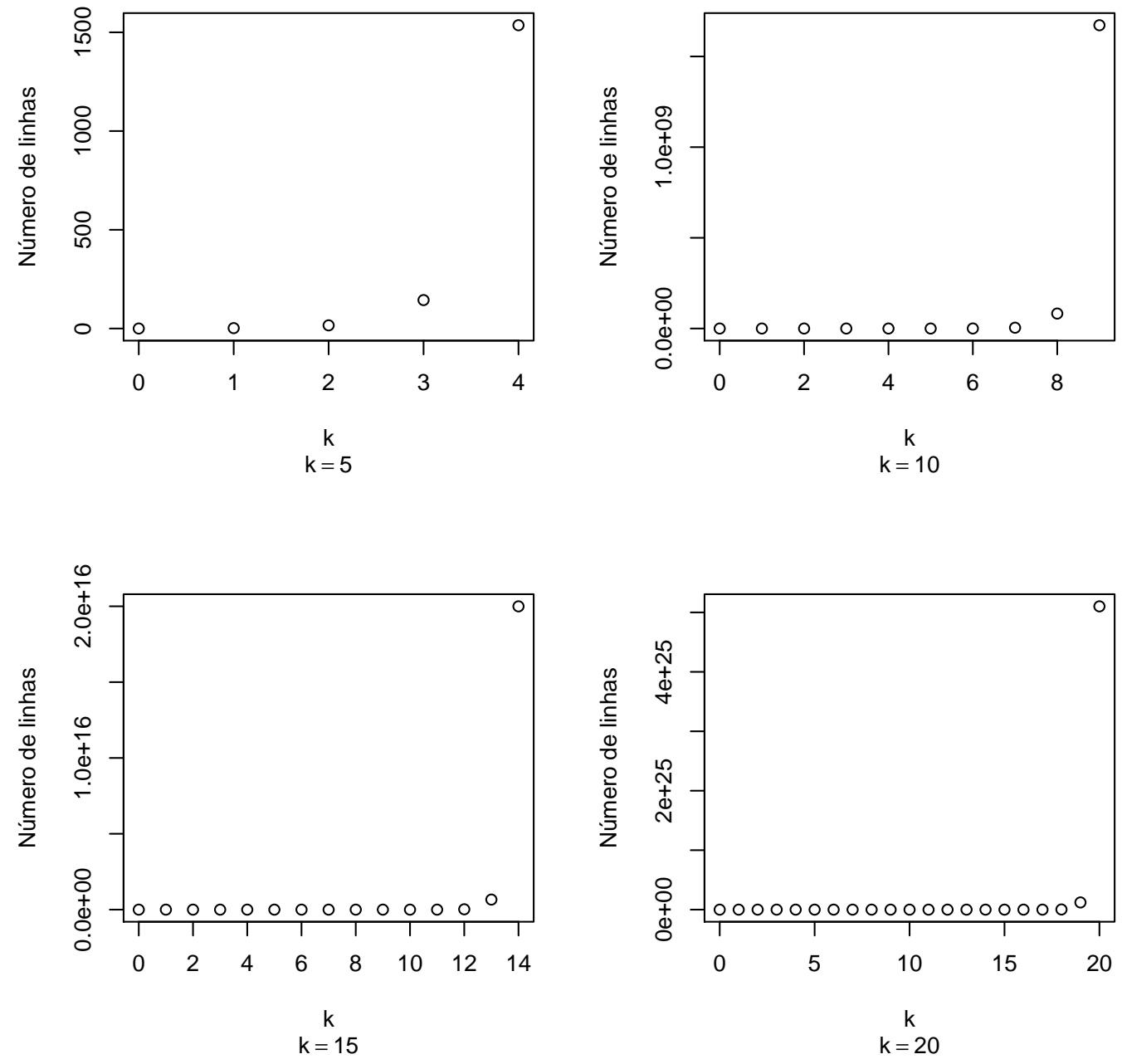

Figura 4.2: Número de linhas da matriz $\mathbb{C}_{k}^{\prime}$ 
1. Fixar um inteiro $n$ relativamente grande;

2. Gerar $\theta_{i} \stackrel{i . i . d .}{\sim} H$ para $i=1, \ldots, n$;

3. Para $i=1, \ldots, n+1$ gerar $E_{i}$ de uma distribuição exponencial com média 1 independente de $\left(\theta_{i}\right)_{1 \leq i \leq n}$ e considere $\Gamma_{i}=E_{1}+\ldots+E_{i}$

4. Calcule $G_{n}^{-1}\left(\Gamma_{i} / \Gamma_{n+1}\right)$,

onde a função $G_{n}^{-1}$ é definida em Zarepour e Labadi (2011) como sendo a função quantílica de uma distribuição $\operatorname{Gama}(\alpha / n, 1)$ aplicada no ponto $1-\Gamma_{i} / \Gamma_{n+1}$, i. e., uma função que retorna o ponto, no domínio de uma variável aleatória qualquer que siga a distribuição gama, que acumula uma probabilidade igual a $1-\Gamma_{i} / \Gamma_{n+1}$.

Por uma questão expositiva, abaixo são apresentados as representações do processo de Dirichlet, a desenvolvida por Ferguson (1973)

$$
P^{\text {Ferg. }}(\cdot)=\sum_{i=1}^{\infty} \frac{N^{-1}\left(\Gamma_{i}\right)}{\sum_{i=1}^{\infty} N^{-1}\left(\Gamma_{i}\right)} \delta_{\theta_{1}}(\cdot)
$$

onde $N(x)=\alpha \int_{x}^{\infty} \exp ^{-t} t^{-1} d t$, para $x>0$ e $\delta_{x}(B)=1$ se $x \in B$ e $\delta_{x}(B)=0$ caso contrario. E a respresentação aproximada, contida em Zarepour e Labadi (2011),

$$
P_{n}^{N e w}(\cdot)=\sum_{i=1}^{n} \frac{G_{n}^{-1}\left(\Gamma_{i} / \Gamma_{n+1}\right)}{\sum_{i=1}^{n} G_{n}^{-1}\left(\Gamma_{i} / \Gamma_{n+1}\right)} \delta_{\theta_{i}}(\cdot) .
$$

Com a aproximação, suge a questão de quantos $\theta_{i}^{\prime} s$ devem ser gerados para que se tenha uma aproximação satisfatória? Qual o menor $n$ que possibilita uma aproximação de tamanho $\epsilon$ ? Este resultado também é definido em Zarepour e Labadi (2011).

$$
n=\min \left\{j: \frac{G_{j}^{-1}\left(\Gamma_{i} / \Gamma_{j+1}\right)}{\sum_{i=1}^{j} G_{j}^{-1}\left(\Gamma_{i} / \Gamma_{j+1}\right)}<\epsilon\right\} .
$$

Em Tsunemi et al. (2012), a medida suporte do processo de Dirichlet é definida como

$$
\mu_{\eta}(A)=\Lambda \int_{A} \gamma \exp ^{-\gamma(t-\eta)} \mathbb{I}_{(\eta, \infty)}(t) d t+\Delta \mathbb{I}_{A}(\infty)
$$

uma medida cujo suporte é a reta estendida, intervalo $(0, \infty]$, e por isso define um peso, $\Lambda$, para o intervalo $(0, \infty)$ e um peso, $\Delta$, no "ponto" infinito.

Note que a variável $\eta$ é uma complicação na geração do processo, devido à dependência da medida suporte no instante em que o processo de produção sai do controle. Se fhhhhhhhhhhhhhhorem geradas observações diretamente de $\mu_{\eta}$ como escrita na equação 4.11, o resultado obtido no final de todo o processo de simulação, serão observações de $F(t) \mid \tau=k, \eta$ e não de $F(t) \mid \tau=k$, o que é desejado.

A solução encontrada para esse problema foi gerar observações de $\eta$, e com essas observações, gerar um $\mu_{\eta}$ para cada $\eta$. Com isso, pode-se extrair a influência de $\eta$, calculando uma simples média, que será considerada como uma amostra de do processo de Dirichlet em questão.

Depois de todas essas observações e comentários sobre os detalhes de se simular um processo de Dirichlet, segue abaixo o algoritmo usado para gerar uma observação desse processo.

O algoritmo 12 gera a medida aleatória do intervalo $[\eta, \infty]=\bigcup_{i=1}^{n}\left(\theta_{i-1}, \theta_{i}\right)$, onde $\theta_{0}$ seria o limite inferior do suporte, $\eta$. Para gerar uma medida aleatória na reta estendida devemos executar o algoritmo várias vezes e então calcular uma média das medidas aleatórias para os vários $\eta$ que foram gerados, eliminando, de certa forma, a influência de $\eta$ na medida suporte do processo. Para tal cálculo é interessante distribuir a medida aleatória de probabilidade dos intervalos $\left(\theta_{i-1}, \theta_{i}\right)$ (para $i$ 


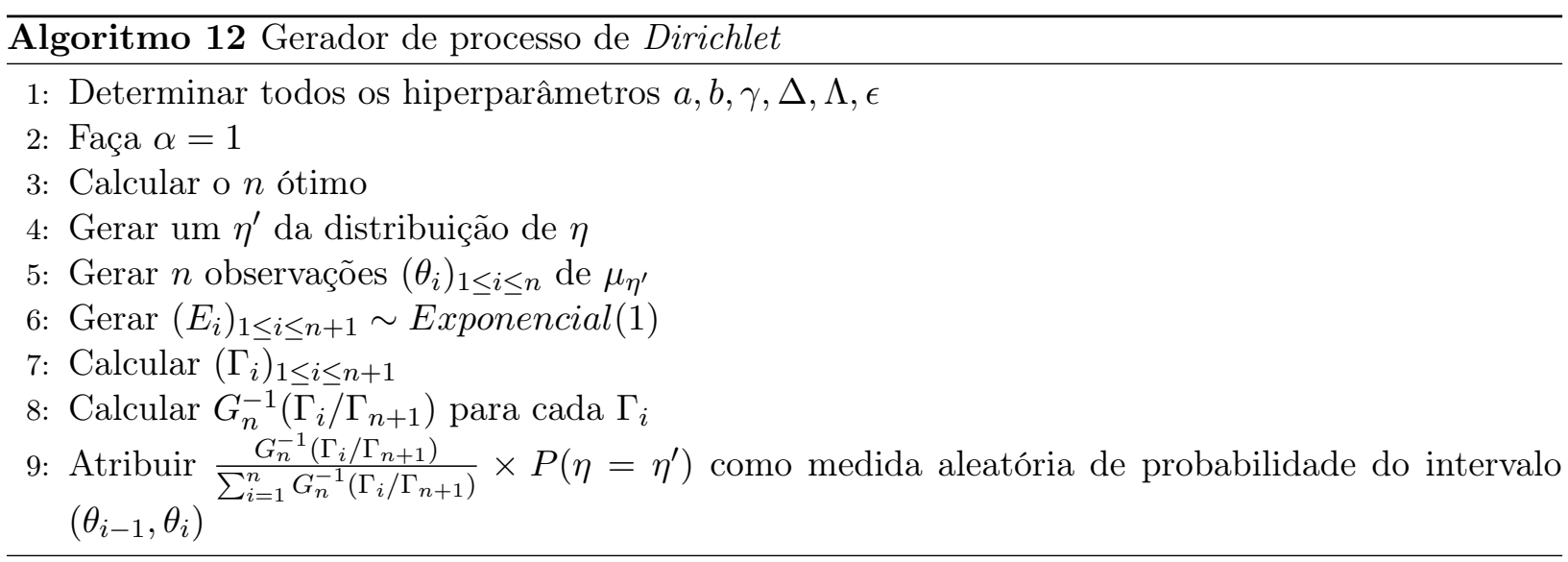

variando de 1 a $n)$ para os intervalos $(j-1, j] \forall j \in\{1, \ldots, \infty\}$. Este procedimento facilita o cálculo da média das várias medidas aleatórias geradas.

Essa média será usada como medida de probabilidade para o processo de controle de qualidade via procedimento de Taguchi, ou seja a probabilidade da se encontrar um item não conforme na $k$-ésima inspeção será a média das várias medidas aleatórias geradas através do algoritmo 12.

\subsubsection{Gerando observações do processo de controle de qualidade.}

O procedimento de Taguchi consiste em inspecionar a última peça de cada sequência de $m$ produzidas. A probabilidade de que na primeira inspeção seja encontrado um item não-conforme será, dada a medida aleatória (comentada na seção anterior), $P(0, m]$. Uma vez que não foi verificado um item não-conforme na primeira inspeção, a probabilidade de que na segunda inspeção se encontre um item não-conforme será a mesma medida aleatória, mas agora avaliada no intervalo $(0,2 m]$, e assim por diante até se encontrar um item não-conforme, como na equação 4.2. A variável $\tau$, descrita antes, assim como $\tau^{\prime}$, presente no algoritmo 13, representam o número de inspeções necessárias para verificar um item não-conforme.

Uma descrição mais clara desse processo é apresentada no algoritmo 13.

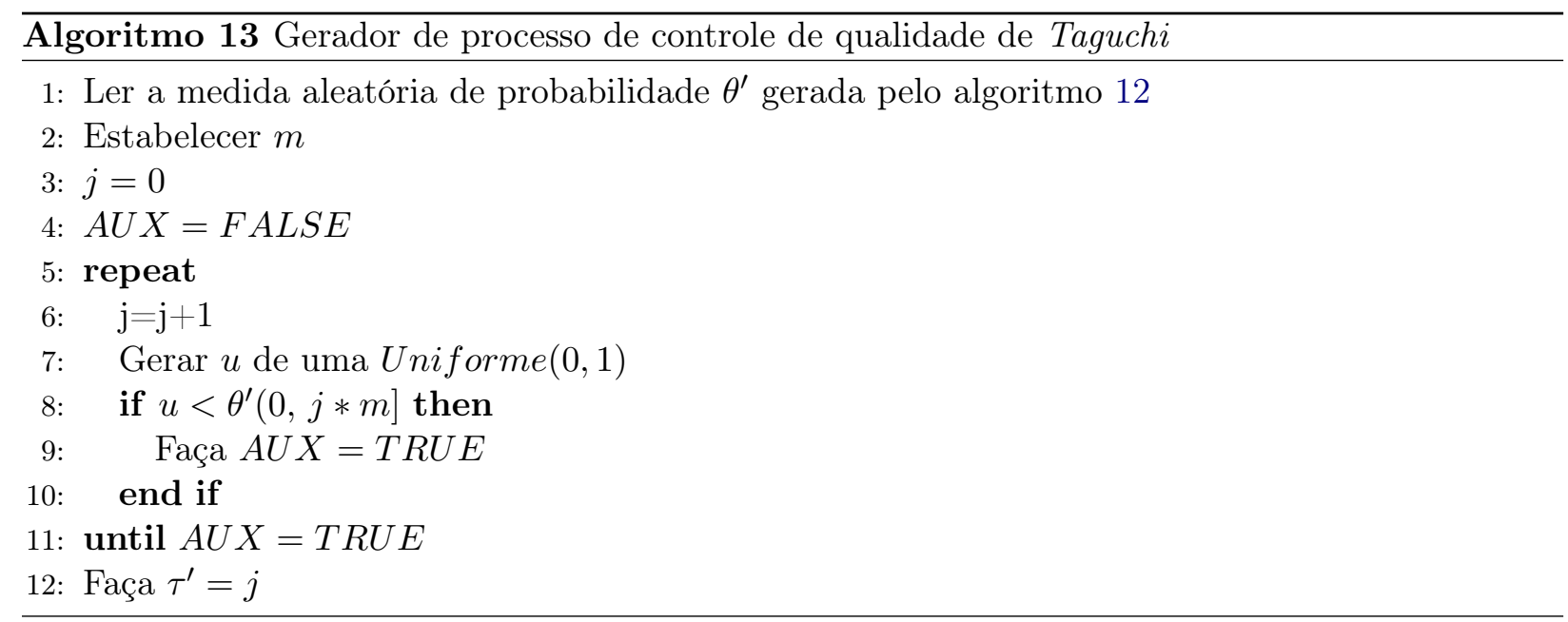

\subsection{3 $A B C$ configurado para gerar uma função de probabilidade acumulada}

De posse dos algoritmos apresentados nas seções anteriores, temos tudo que é necessário para aplicar um algoritmo $\boldsymbol{A} \boldsymbol{B} \boldsymbol{C}$ ao modelo de Tsunemi et al. (2012). Já se sabe como gerar observações da priori e como replicar o experimento.

Como a variável observada tem como domínio o conjunto dos números naturais (o instante em que foi detectado um item não-conforme), então os parâmetros de tuning $(\rho(\cdot), \eta(\cdot)$ e $\epsilon)$ não são necessários. 
Depois de ter simulado um processo de inspeção de Taguchi e verificado um item não-conforme, determinando assim uma inspeção simulada $\left(\tau^{\prime}\right)$, compara-se esse $\tau^{\prime}$ com a inspeção observada $(\tau)$. Se as duas inspeções forem iguais, então a medida aleatória de probabilidade, que gerou o processo de inspeção simulado, é aceita como uma observação da distribuição a posteriori, equação 4.4.

Uma vez que uma amostra, suficientemente grande da distribuição de probabilidade a posteriori de medidas aleatórias de probabilidade, foi gerada, é necessário calcular o estimador de Bayes, sob perda quadrática, da proporção de itens não-conformes ao longo do processo, F, objetivo principal do trabalho de Tsunemi et al. (2012). Como é sabido, o estimador em questão é a média da distribuição a posteriori. Assim, basta calcular a média das medidas aleatórias de probabilidade geradas pelo $\boldsymbol{A} \boldsymbol{B} \boldsymbol{C}$, nos intervalos $(i-1, i]$, para $i=1,2, \ldots$ Esse processo de estimação está transcrito no algoritmo 14. Note que esse algoritmo é junção dos algoritmos 12 e 13

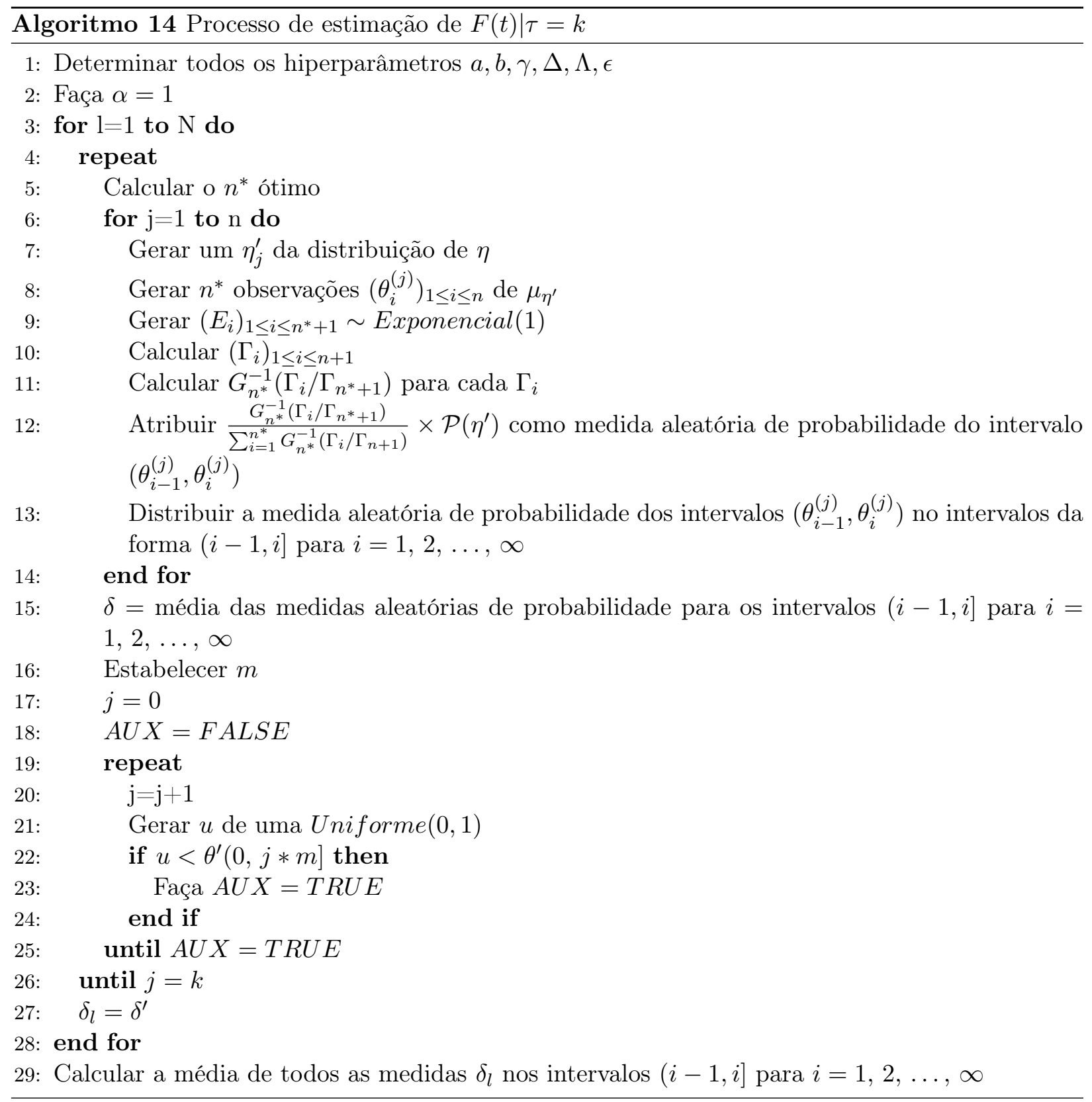

Para calcular a média de todos as medidas $\delta_{l}$ (Definido no algoritmo 14) nos intervalos $(i-1, i]$ para $i=1,2, \ldots, \infty$, primeiro distribui-se cada medida $\delta_{l}$ nos intervalos já citados, obtendo assim $\delta_{l}(i-1, i]$ para $i=1,2, \ldots, \infty$. Então é calculada a média de cada $\delta_{l}(i-1, i] \forall i, l$. 


\subsubsection{Escolha do método para gerar uma observação de um processo de Dirichlet}

Para fins comparativos, aqui será apresentado um comparativo das amostras geradas pelo $\boldsymbol{A B C}$ para o modelo em estudo, mas utilizando-se diferentes métodos para gerar observações da distribuição a priori.

O método Rapid Simulation of a Dirichlet Process será, aqui, comparado com o stickbreaker. O método que obtiver melhor desempenho será utilizado para gerar observações da distribuição 4.4, e por consequência, obter estimativas de $F(t) \mid \tau=k$.

Uma comparação das estimativas da proporção de itens não-conformes ao longo do processo de produção, usando os dois métodos de simulação de um processo de Dirichlet é apresentada na Figura 4.4. Ambas as estimativas foram calculadas considerando $\tau=3$ e todos os hiperparâmetros iguais a 5. Foram geradas 10 amostras de tamanho 100, e o número de interações necessárias para se gerar uma amostra foi anotado. As estatísticas descritivas do número de interações se encontra na Tabela 4.1 e na Figura 4.3

Tabela 4.1: Estatísticas descritivas do número de interações que o $\boldsymbol{A B C}$ levou para gerar as estimativas de $F(t) \mid \tau=k$, usando tanto o Stickbreaker como o Rapid Simulation

\begin{tabular}{c|cc}
\hline \hline & Rapid Simulation & Stickbreaker \\
\hline Média & 744.90 & 1253.20 \\
Mínimo & 649.00 & 1153.00 \\
$1^{\circ}$ Quartil & 694.00 & 1176.50 \\
Mediana & 740.50 & 1234.00 \\
$3^{\circ}$ Quartil & 783.25 & 1320.75 \\
Máximo & 856.00 & 1394.00 \\
Desvio Padrão & 70.90 & 86.92 \\
Variância & 5026.99 & 7554.62 \\
\hline \hline
\end{tabular}

As informações contidas no gráfico 4.3 e na tabela 4.1 reforçam os argumentos de Zarepour e Labadi (2011) afirmando que seu método é o mais rápido que o stickbreaker na simulação de um processo de Dirichlet. Espera-se que também seja o método com a melhor aproximação das amostras do presente modelo em estudo.

É possível ver (Figura 4.4) que os valores das estimativas geradas pelo $\boldsymbol{A B C}$, usando o stickbreaker como gerador da priori, estão em um patamar maior que os valores das estimativas geradas pelo $\boldsymbol{A B C}$, quando é utilizado o Rapid Simulation.

Comparando esses resultados, com os que se encontram em Tsunemi et al. (2012, páginas 2705 e 2706), tem-se a impressão de que as estimativas geradas através do stickbreaker são superestimadas, gerando valores em um patamar maior que o esperado.

Com base nessas informações, maior velocidade do Rapid Simulation, aliado com a suspeita de superestimar a proporção de itens não-conformes do stikbreaker, o método escolhido para gerar amostras de um processo de Dirichlet foi o Rapid Simulation of a Dirichilet Process.

\subsection{Calculando a estimativa da proporção dos itens não-conforme ao longo do processo de produção usando o amostrador de Gibbs}

Como citado em Tsunemi (2009), Calle e Gómez (2001) propuseram uma metodologia baseada no amostrador de Gibbs para obter o estimador bayesiano não-paramétrico da função de sobrevivência para o caso de censura intervalar quando a priori é um processo de Dirichlet.

O que é feito em Calle e Gómez (2001) é introduzir uma variável latente no modelo, estudado neste trabalho, e então configurar um amostrador de Gibbs. Calle e Gómez propõem, para gerar amostras do vetor $w$, dada as observações $D$, a introdução de um vetor $n$ como uma variável latente. Então denota-se $[n \mid w, D]$ como a distribuição a posteriori do vetor $n$ condicionado ao vetor $w$ e, $[w \mid n, D]$ a distribuição a posteriori do vetor $w$ condicionado ao vetor $n$.

Tendo posse de $[n \mid w, D]$ e $[w \mid n, D]$, o amostrador de Gibbs é da seguinte forma, algoritmo 15. 


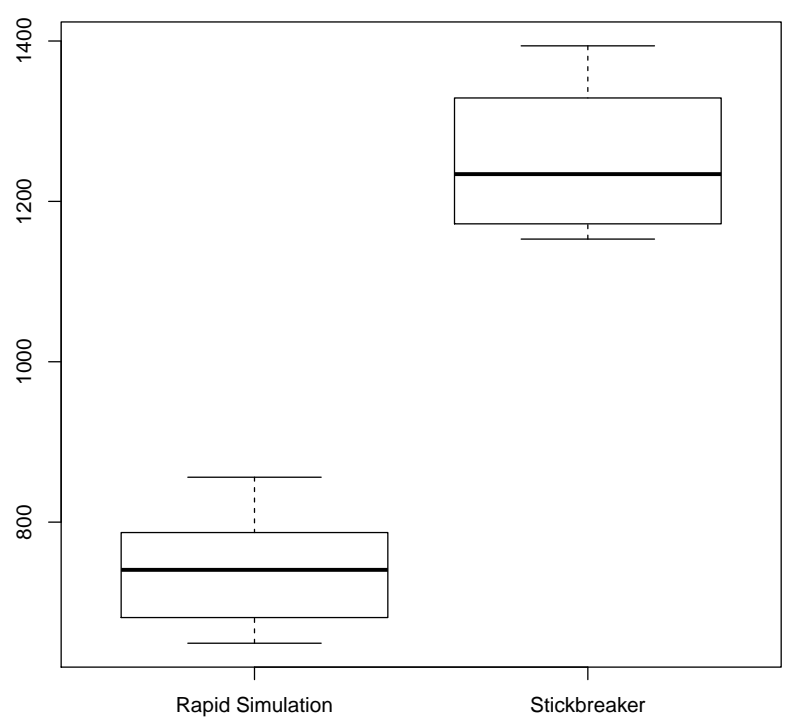

Figura 4.3: Boxplot do numero de interações necessárias para gerar amostras utilizando o stickbreacker e o Rapid Simulation, como amostradores da distribuição priori

Como no modelo apresentado em Tsunemi et al. (2012) já existe uma variável latente, $\eta$ e já conhecemos sua distribuição a posteriori, equação 4.7. A variável de interesse, $\left(\mathcal{P}\left(B_{1}\right), \ldots, \mathcal{P}\left(B_{n}\right)\right)$, possui uma distribuição que, por construção, já é condicionada em $\eta$, equação 4.4, e, também por construção, $\eta$ é independente de $\left(\mathcal{P}\left(B_{1}\right), \ldots, \mathcal{P}\left(B_{n}\right)\right)$. Assim, o amostrador de Gibbs, para gerar amostras da distribuição 4.4 é o seguinte (algoritmo 16 ).

Aparentemente é bem mais simples gerar observações de 4.4 via amostrador de Gibbs do que usar o $\boldsymbol{A B C}$, mas como já foi mostrado anteriormente, a complexidade matemática para executar os passos 4 e 5 do algoritmo 16 aumenta, conforme $\tau$ aumenta, o que possibilita o seu funcionamento apenas para valores pequenos de $\tau$.

A menor complexidade possível é atingida quando $\tau=1 \mathrm{e}$, é nesse caso em que será executada uma comparação entre o amostrador de Gibbs e o $\boldsymbol{A B C}$.

Também serão comparadas as estimativas criadas pelos dois métodos com as estimativas obtidas pela equação 4.4. É bom lembrar que isso é possível, apenas, por se usar $\tau=1$, pois qualquer outro valor de $\tau$ elevaria muito a complexidade para calcular as estimativas de $F(t) \mid \tau$.

Na figura 4.5 é possível ver as estimativas dos três estimadores (exato, via Gibbs e $\boldsymbol{A} \boldsymbol{B C}$ ) comparadas. Observa-se que as estimativas obtidas através do $\boldsymbol{A} \boldsymbol{B} \boldsymbol{C}$ não se aproximam das estimativas alvo como as que foram geradas pelo algoritmo de Gibbs, que coincidem numericamente com as es- 

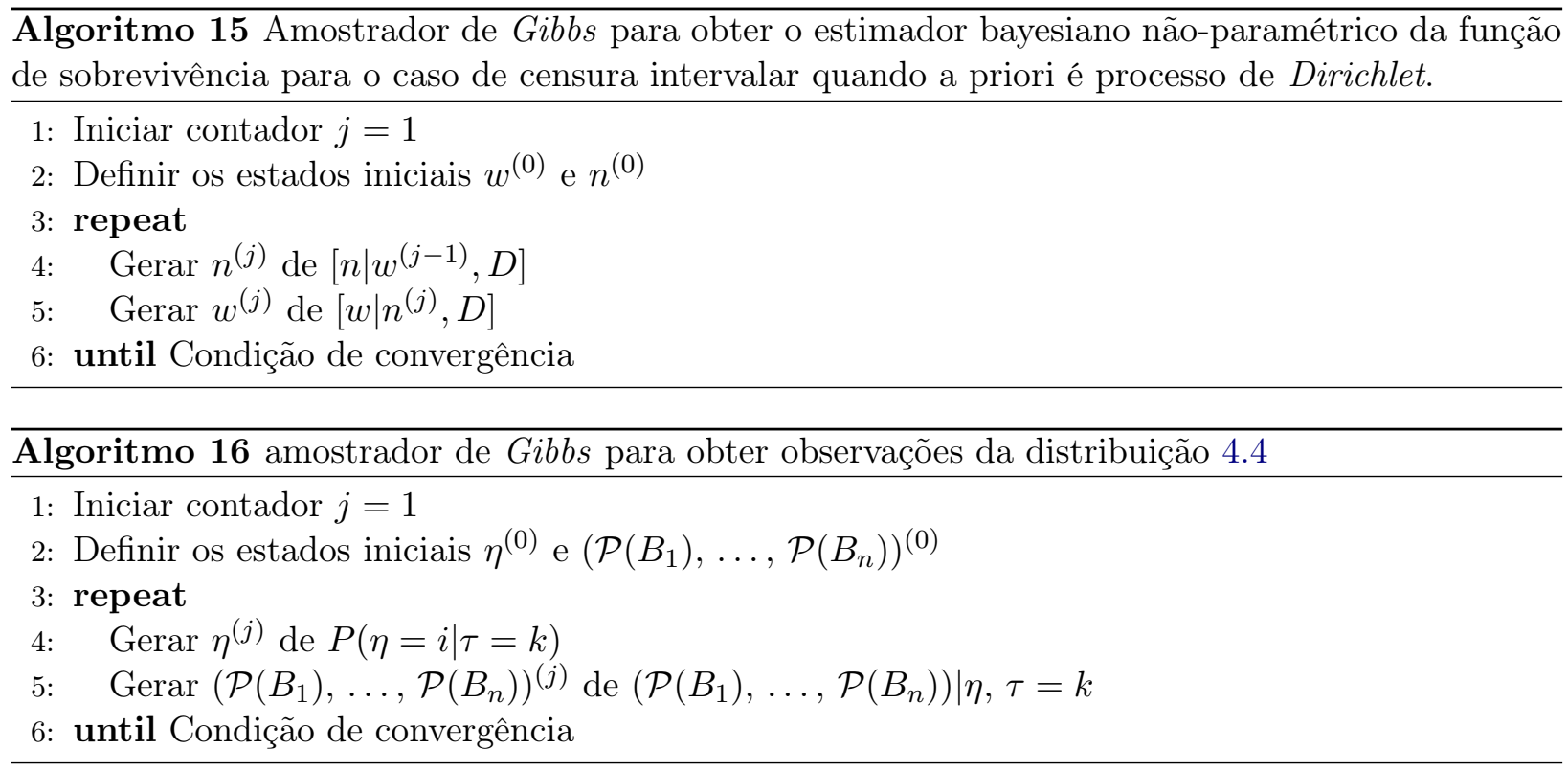

timativas alvo. Esse fato pesa negativamente para o $\boldsymbol{A} \boldsymbol{B C}$. Uma motivo para tal fato ter acontecido é a tendência em gerar amostras em baixa região de probabilidade, comentada anteriormente, que o $\boldsymbol{A} \boldsymbol{B C}$ possui, em particular a versão mais simples do $\boldsymbol{A B C}$, o $\boldsymbol{A} \boldsymbol{B} \boldsymbol{C}$-rejeição, que foi a utilizada para gerar gerar essas estimativas.

As estimativas geradas pelo amostrador de Gibbs coincidiram com as estimativas exatas, alvo, mas tal fato só se fez possível porque estipulou-se $\tau=1$. Para qualquer outro valor de $\tau$ a complexidade do problema, e a complexidade do amostrador de Gibbs, são aumentadas, podendo chegar a um patamar que inviabilize a sua utilização.

Mesmo com esse revés, o $\boldsymbol{A} \boldsymbol{B} \boldsymbol{C}$ continuará sendo utilizado, pois deseja-se investigar o comportamento das estimativas de $F(t) \mid \tau=k$ para outros valores de $k$, o que é, computacionalmente muito difícil usando algoritmos do tipo $M C M C$.

\subsection{Resultados das simulações do $A B C$}

Depois de todas as considerações e análises feitas sobre o $\boldsymbol{A} \boldsymbol{B} \boldsymbol{C}$, serão apresentadas aqui as estimativas de Bayes, sob perda quadrática, da proporção de itens não-conformes ao longo do processo de produção.

As inspeções feitas nos itens produzidos tinham um espaçamento (intervalo entre inspeções) de cinco peças. Os hiperparâmetros fixados nos valores $a=b=\gamma=\Lambda=\Delta=5$. As estimativas de $F(t) \mid \tau=k$ foram calculadas para $\tau=1, \ldots, 10$, ultrapassando a barreira compultacional imposta ao estimador exato 4.6 e, por consequência, ao amostrador de Gibbs.

As estimativas de $F(t) \mid \tau=k$ obtidas através do $\boldsymbol{A} \boldsymbol{B} \boldsymbol{C}$ tem as mesmas propriedades das estimativas encontradas em Tsunemi et al. (2012). À medida que $\tau$ cresce a curva vai se estabilizando em um patamar cada vez menor, como mostrado na Figura 4.6.

Este fato depõe a favor do $\boldsymbol{A B C}$. Dependendo da situação em que o $\boldsymbol{A} B \boldsymbol{C}$ está sendo empregado, o fato dele gerar estimativas de $F(t) \mid \tau=k$ superestimadas também pode ser positivo.

A utilização do $\boldsymbol{A} \boldsymbol{B} \boldsymbol{C}$ não deve ser descartada apenas por superestimar a proporção de itens não-conformes, pois ele permite supere as dificuldades computacionais que o modelo descrito no teorema 4.2.1 tem.

\subsubsection{Análise dos hiperâmetros}

Com o advento do $\boldsymbol{A B C}$ é possível estudar como a variação dos hiperparâmetros influencia nas estimativas da proporção de itens não-conformes, já que, até então, não era viável realizar essa análise utilizando o estimador exato, ou estimativas aproximadas vai amostrador de Gibbs.

Acredita-se que mesmo a aproximação das amostras geradas pelo $\boldsymbol{A} B \boldsymbol{C}$ não sendo tão boa, não vá influenciar na maneira em como uma variação em um hiperparâmetro vá influenciar o estimador. 
Novamente, são fixados $a=b=\gamma=\Lambda=\Delta=5$ e o valor de cada um dos hiperparâmetros é variado, de forma isolada (Figuras 4.7 a 4.11). Também foi fixado $\tau=1$ para cada um dos casos abordados.

Os hiperparâmetros $a$ e $b$, referem-se a opinião à priori do instante em que o processo saiu de controle. Relembrando, $\eta$ é o instante em que o processo de produção sai de controle e $\eta \mid \rho \sim$ Geomtrica $(\rho)$ e $\rho \sim \operatorname{Beta}(a, b)$. Quando somente $a$ está próximo de zero (primeiro caso, figura 4.7), a esperança de $\rho$ também está próxima de zero e, por consequência, a esperança de $\eta\left(E(\eta)=\rho^{-1}\right)$ tende a valores muito grandes. Essa característica está representada na Figura 4.7. Note que as curvas crescem de maneira cada vez menos suavizada e se estabilizam em valores de $t$ cada vez maiores.

Já para quando somente $b$ está próximo de zero, a esperança de $\rho$ é um número muito grande, e, assim, a esperança de $\eta$ é um número muito pequeno. É possível ver na Figura 4.8 as curvas estabilizam-se em valores de $t$ menores conforme diminuimos o valor de $\mathrm{b}$.

Os outros parâmetros, $\gamma, \Lambda$, e $\Delta$, são relacionados à medida suporte do processo de Dirichlet. Como mostrado anteriormente, a medida suporte do processo é uma mistura de outras duas medidas. Uma medida degenerada no infinito positivo e uma medida exponencial na reta real. Lembrando também que a medida $\mu$ depende de $\eta$, pois $\mu_{\eta}$ só assume valores positivos no intervalo $(\eta, \infty]$. Os pesos das medidas exponencial na reta real e degenerada no ponto infinito são, respectivamente, $\Lambda$ e $\Delta$. O hiperparâmetro $\gamma$, o mesmo que o parâmetro de uma distribuição exponencial qualquer, representa, dentre outras coisas, o quanto a medida $\mu_{\eta}$ é dispersa na reta real. Para $\gamma$ grande, a medida exponencial é mais concentrada nas proximidades de $\eta$ e quando $\gamma$ é pequeno, a medida se espalha por mais regiões da reta real. Essa propriedade não é fácil de perceber, pois a variação nas curvas é pequena com relação a variação em $\gamma$, mas está presente nas curvas da Figura 4.9.

A Figura 4.10 nos mostra como um pequeno valor de $\Lambda$ afeta as estimativas de $F(t) \mid \tau=1$. Note que ao diminuir a medida na reta real, em relação a medida degenerada no infinito, o valor em que as curvas se estabilizam também diminui. E quando diminui-se o peso da medida degenerada no infinito, com relação a medida na reta real, o valor em que as curvas se estabilizam aumenta (Figura 4.11).

\subsection{Inferência preditiva}

Após a estimação das quantidades desconhecidas, $\left(\mathcal{P}\left(B_{1}\right), \ldots, \mathcal{P}\left(B_{n}\right)\right)$, vetor de probabilidades aleatórias, o passo seguinte na inferência estatística, como já comentado e apresentado anterior, é a predição de novas observações, dada as observações passadas.

No contexto do do modelo abordado aqui, manipular a distribuição de probabilidade a posteriori (equação 4.5), a fim de obter a função de probabilidade preditiva, é extremamente difícil.

Novamente a solução é obter a função de probabilidade preditiva de maneira numérica.

Serão usadas as amostras geradas pelo $\boldsymbol{A} \boldsymbol{B} \boldsymbol{C}$, as mesmas utilizadas para calcular as estimativas encontradas na figura 4.6, para calcular, de forma numérica, $f\left(x_{n+1} \mid x_{1}, \ldots, x_{n}\right)$ usando a mesma técnica utilizada no caso do ultimo capítulo.

Na tabela 4.2 é apresentada a distribuição de probabilidade preditiva do número de inspeções necessárias para detectar um item não-conforme, num futuro ciclo de produção, dado a inspeção que detectou um item não-conforme no atual ciclo de produção. É possível ver que é mais provável encontrar itens não-conforme nas primeiras inspeções, mesmo quando, num ciclo de produção anterior, foi encontrado um item não-conforme na decima inspeção. Tal fato pode ser creditado a medida suporte do processo de Dirichlet e os outros hiperparâmetros supostos, pois eles concentram a medida de probabilidade em valores pequenos do intervalo $(0, \infty]$.

Os valores da tabela 4.2 foram arredondados para duas casas decimais, portanto os valores iguais a zero, presentes na tabela, são valores menores que $10^{-2}$.

\subsection{Observações e considerações finais}

Tsunemi (2009) e Tsunemi et al. (2012) introduziram a abordagem Bayesiana na estratégia de monitoramento de Taguchi. O modelo proposto nesses trabalhos é a primeira tentativa de resolver 
Tabela 4.2: Distribuição preditiva do número de inspeções necessárias para detectar um item não-conforme, num próximo ciclo de produção

\begin{tabular}{ccccccccccc}
\hline \hline \multirow{2}{*}{$\tau$} & \multicolumn{10}{c}{ Inspeções do próximo ciclo de produção } \\
\cline { 2 - 12 } & 1 & 2 & 3 & 4 & 5 & 6 & 7 & 8 & 9 & 10 \\
\hline 1 & 0.46 & 0.38 & 0.12 & 0.03 & 0.01 & 0.00 & 0.00 & 0.00 & 0.00 & 0.00 \\
2 & 0.41 & 0.41 & 0.14 & 0.03 & 0.01 & 0.00 & 0.00 & 0.00 & 0.00 & 0.00 \\
3 & 0.39 & 0.37 & 0.16 & 0.05 & 0.02 & 0.01 & 0.00 & 0.00 & 0.00 & 0.00 \\
4 & 0.34 & 0.36 & 0.19 & 0.07 & 0.03 & 0.01 & 0.00 & 0.00 & 0.00 & 0.00 \\
5 & 0.33 & 0.35 & 0.19 & 0.07 & 0.03 & 0.01 & 0.01 & 0.00 & 0.00 & 0.00 \\
6 & 0.31 & 0.34 & 0.20 & 0.09 & 0.04 & 0.02 & 0.01 & 0.00 & 0.00 & 0.00 \\
7 & 0.28 & 0.34 & 0.21 & 0.10 & 0.04 & 0.02 & 0.01 & 0.00 & 0.00 & 0.00 \\
8 & 0.27 & 0.32 & 0.20 & 0.10 & 0.05 & 0.03 & 0.01 & 0.01 & 0.00 & 0.00 \\
9 & 0.27 & 0.30 & 0.20 & 0.11 & 0.06 & 0.03 & 0.01 & 0.01 & 0.00 & 0.00 \\
10 & 0.22 & 0.29 & 0.21 & 0.13 & 0.07 & 0.04 & 0.02 & 0.01 & 0.01 & 0.00 \\
\hline \hline
\end{tabular}

o problema de estimação da proporção de itens não conformes durante um ciclo do processo de produção, e assim, permitir estudar o problema de recall após a produção.

Como todo modelo inicial, sempre há pontos a serem melhorados. Na seção 4.2.3, foi abordada sua limitação computacional. A utilização do $\boldsymbol{A B C}$ para calcular as estimativas da proporção de itens não-conformes durante o processo de produção supera a limitação computacional do modelo 4.6, gerando estimativas com uma aproximação não tão boa quanto as estimativas geradas pelo algoritmo de Gibbs, mas ainda assim aceitáveis, e com um tempo de processamento muito menor, possibilitando a geração de grandes amostras, a análise das mesmas, além de obter a distribuição preditiva da número de inspeções até detectar um item não-conforme.

Apesar do avanço computacional que o $\boldsymbol{A} \boldsymbol{B} \boldsymbol{C}$-Rejeição (versão utilizada aqui) proporciona, ele ainda é um método de amostragem baseado em rejeição. É possível haver combinações específicas de $a, b, \gamma, \Lambda, \Delta$, e $\tau$ que vão diminuir a probabilidade de aceitação. Esse fato leva a uma grande quantidade de interações necessárias para gerar uma amostra da distribuição a posteriori, o que pode impossibilitar o processo inferencial. 


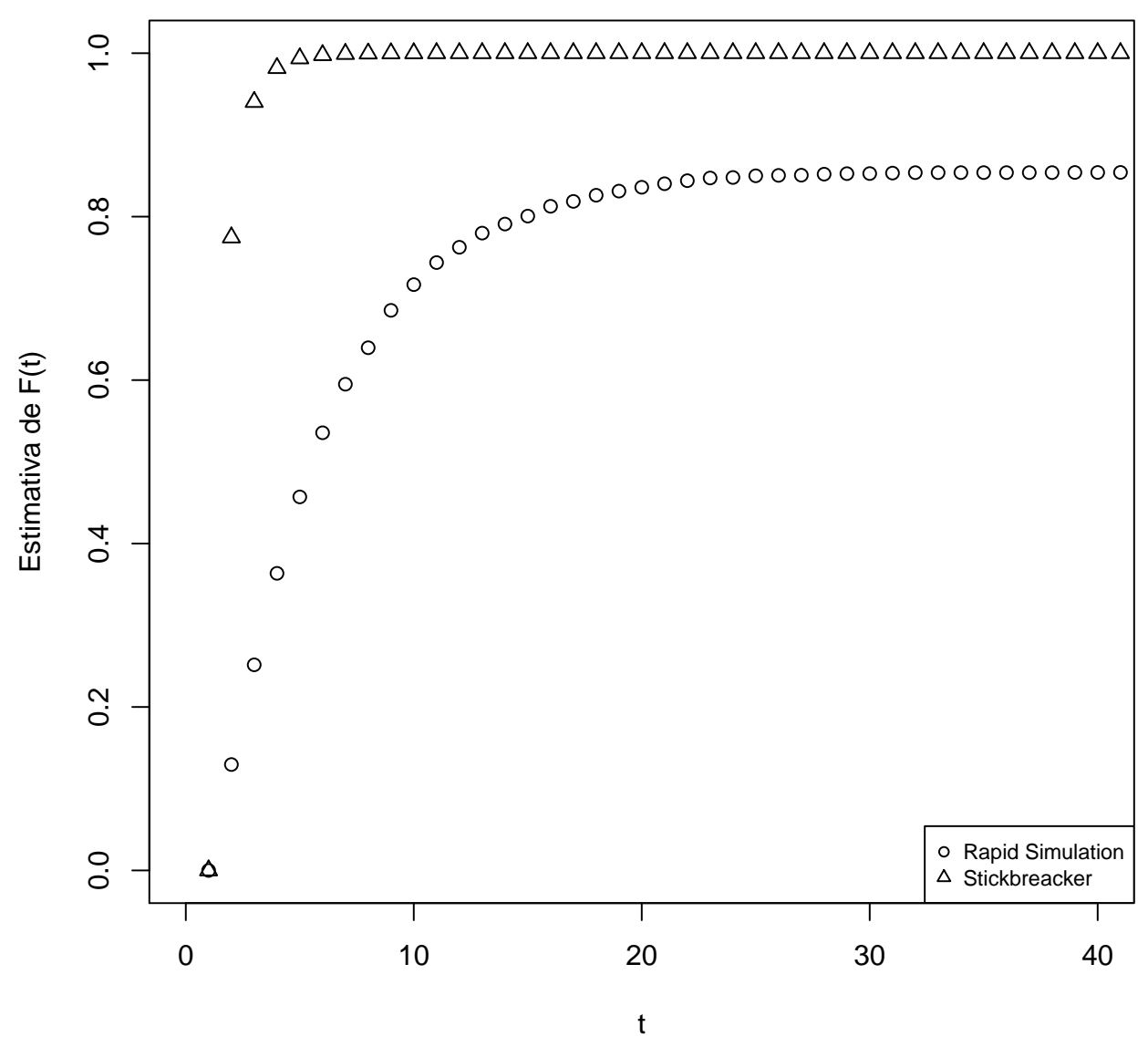

Figura 4.4: Estimativas de $F(t) \mid \tau=k$ geradas pelo $\boldsymbol{A B C}$, usando o Stickbreaker e o Rapid Simulations Of A Dirichlet Process 
$60 \boldsymbol{A} \boldsymbol{B} \boldsymbol{C}$ APLICADO AO MODELO BAYESIANO NÃO-PARAMÉTRICO PARA O MONITORAMENTO "ON-LINE" DE QUALIDADE DE TAGUCHI PARA ATRIBUTOS.

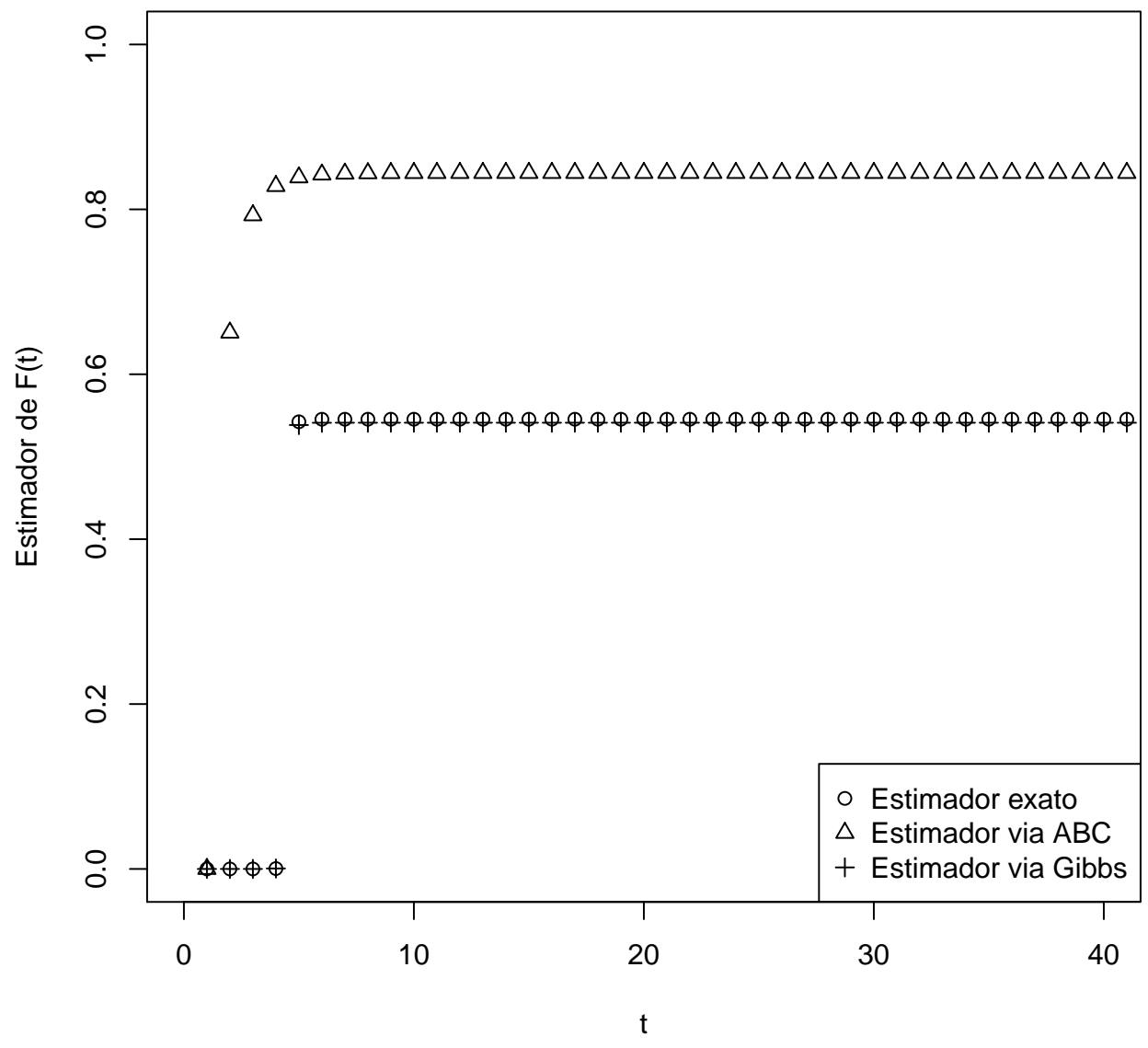

Figura 4.5: Estimativas de $F(t) \mid \tau=1$, obtidos pela equação 4.4, pelo $\boldsymbol{A B C}$ (algoritmo 14) $e$ Gibbs(algorimo 16) 


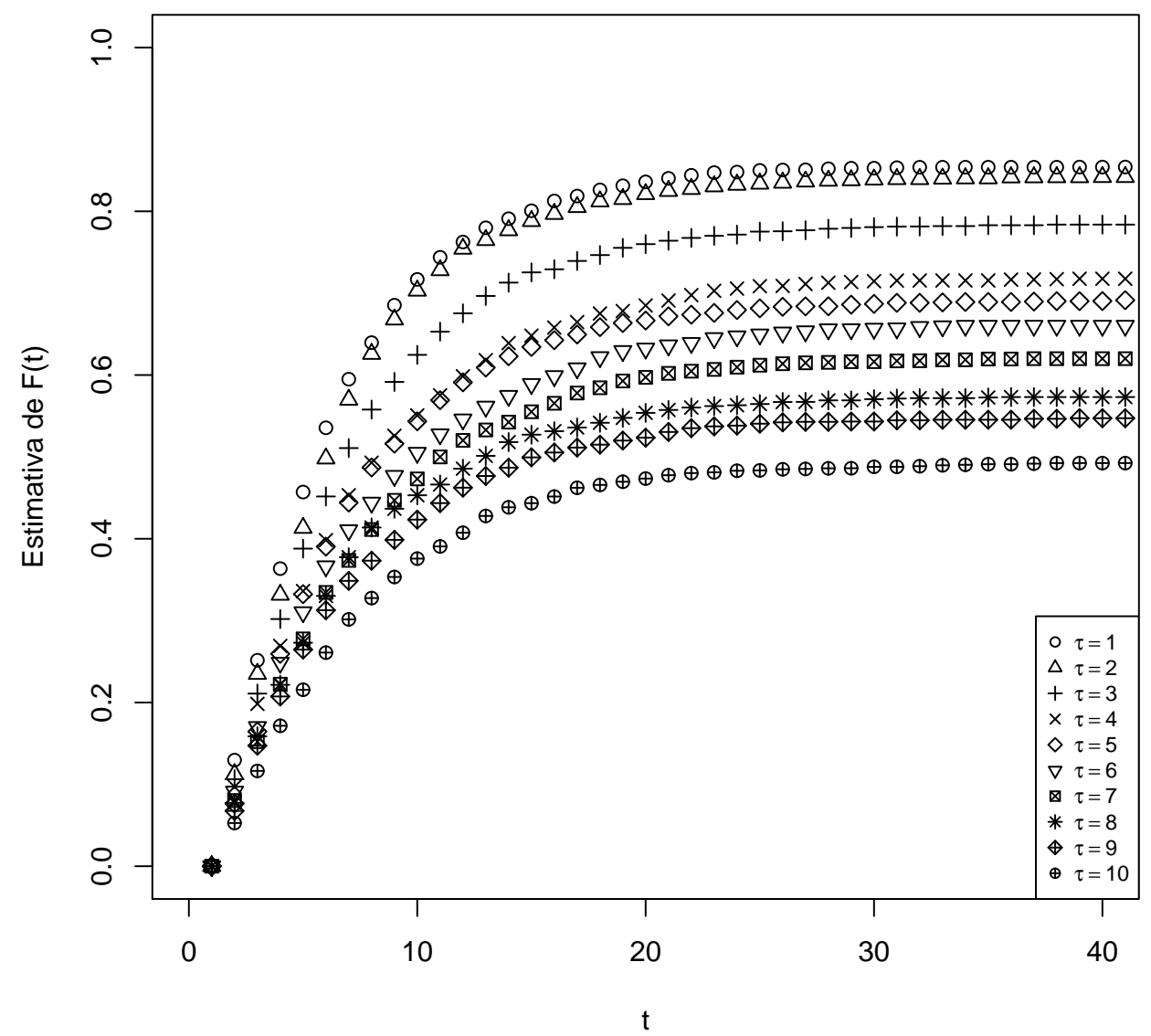

Figura 4.6: Estimativas de $F(t) \mid \tau=k$ para $a=b=\gamma=\Lambda=\Delta=5$, dado $\tau=k$ para $k=1, \ldots, 10$ 
$62 \boldsymbol{A} \boldsymbol{B} \boldsymbol{C}$ APLICADO AO MODELO BAYESIANO NÃO-PARAMÉTRICO PARA O MONITORAMENTO "ON-LINE" DE QUALIDADE DE TAGUCHI PARA ATRIBUTOS.

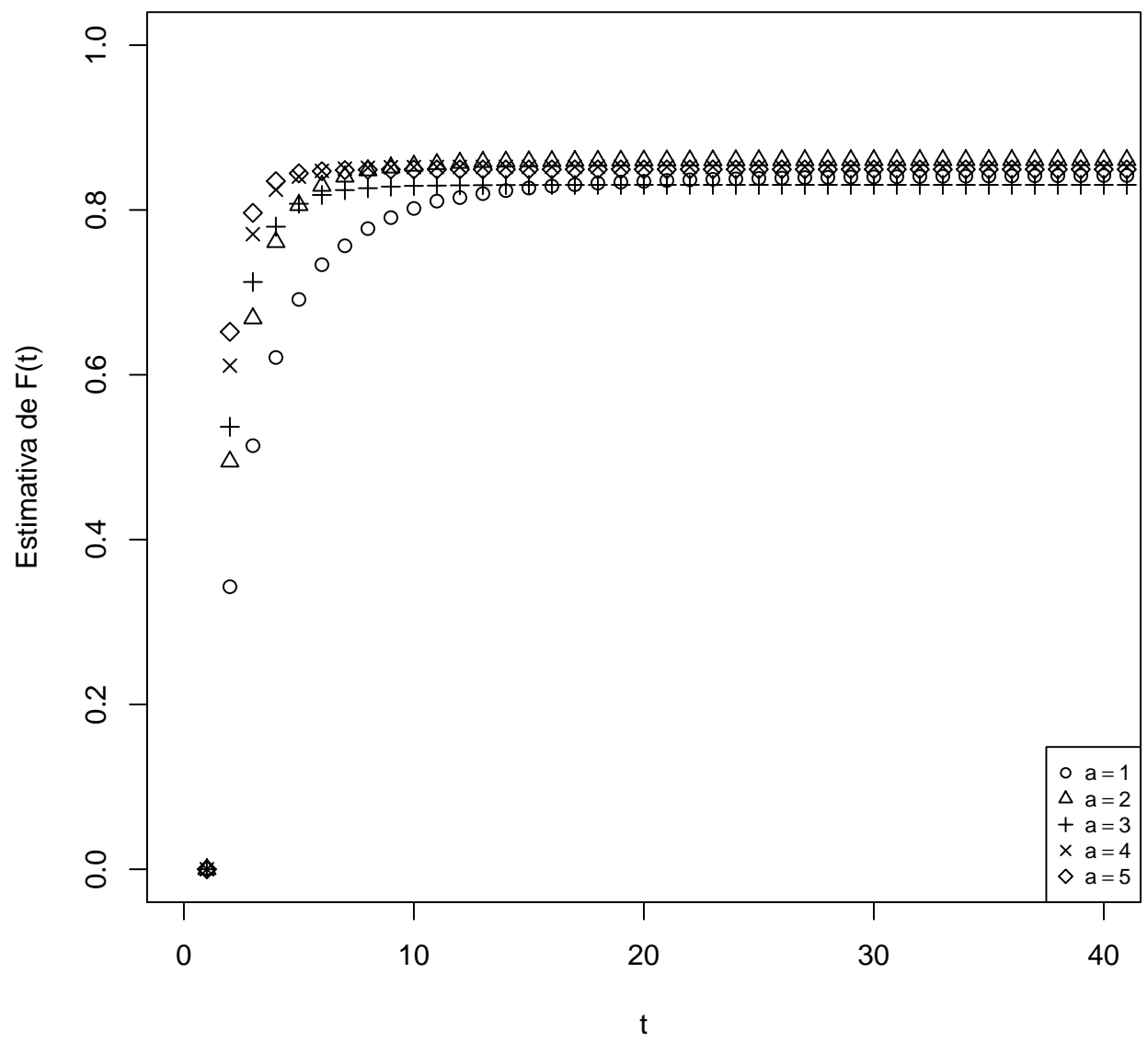

Figura 4.7: Estimativas de $F(t) \mid \tau=1$ variando de acordo com valores de a 


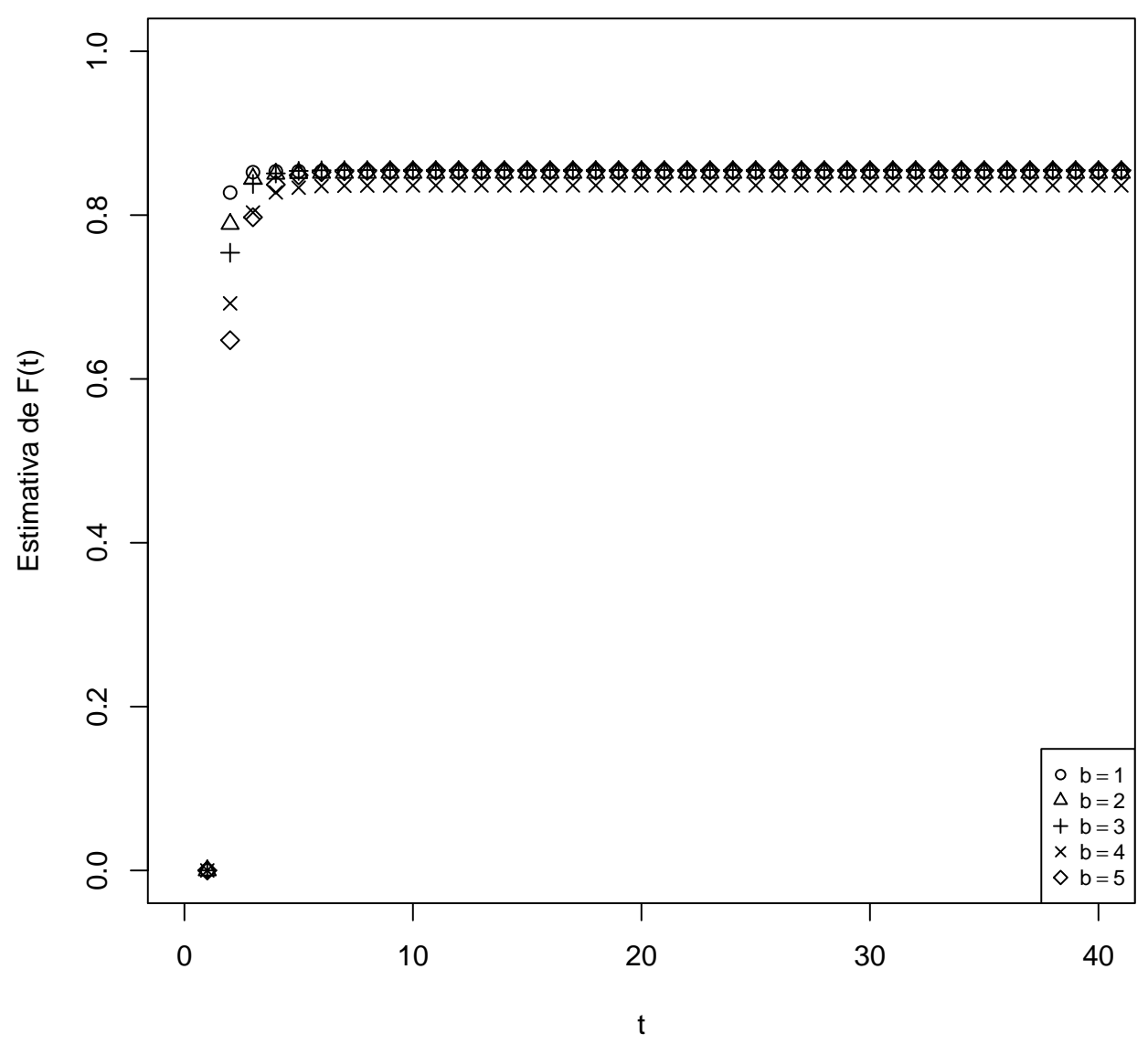

Figura 4.8: Estimativas de $F(t) \mid \tau=1$ variando de acordo com valores de $b$ 
$64 \boldsymbol{A} \boldsymbol{B} \boldsymbol{C}$ APLICADO AO MODELO BAYESIANO NÃO-PARAMÉTRICO PARA O MONITORAMENTO "ON-LINE" DE QUALIDADE DE TAGUCHI PARA ATRIBUTOS.

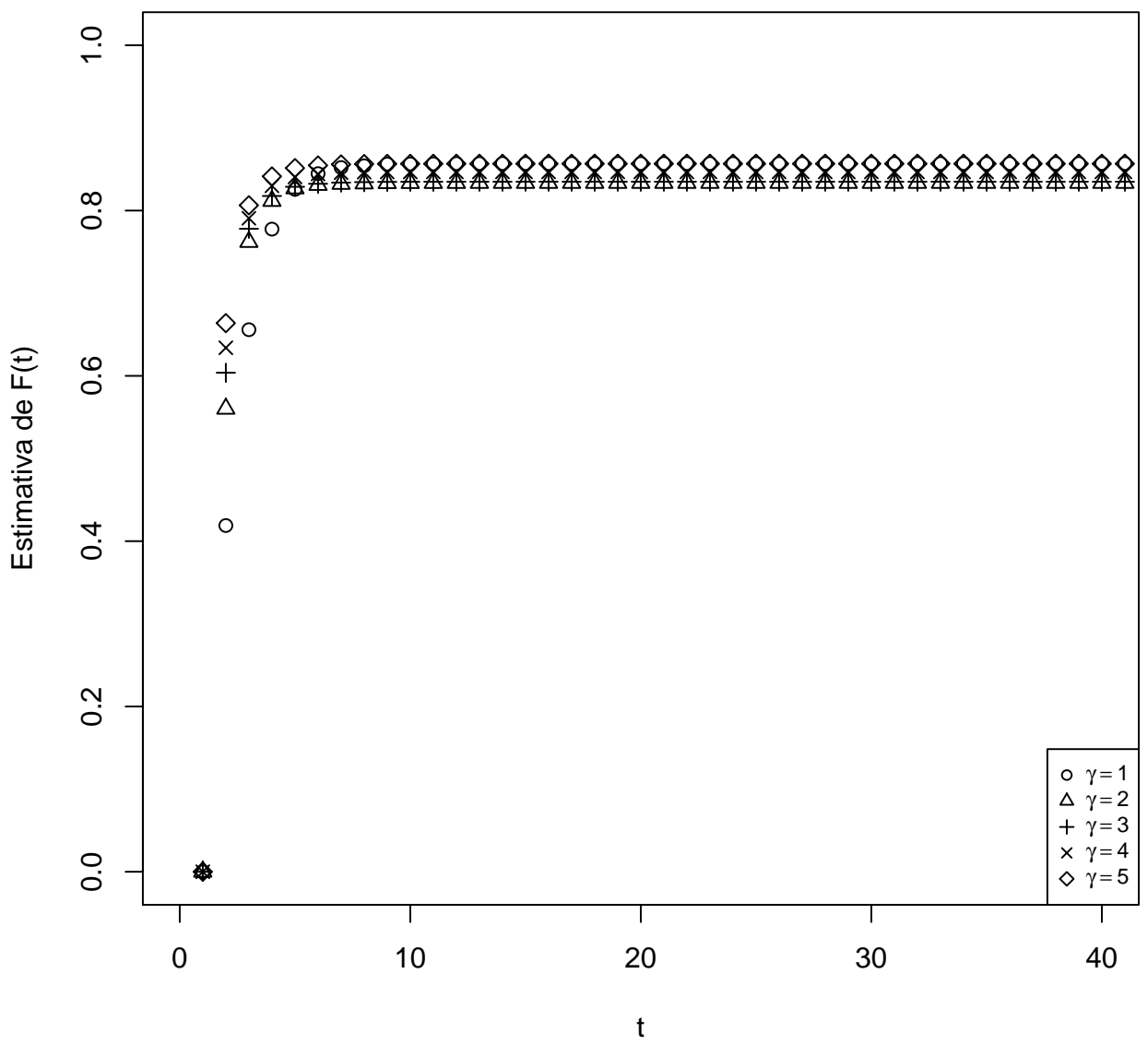

Figura 4.9: Estimativas de $F(t) \mid \tau=1$ variando de acordo com valores de $\gamma$ 


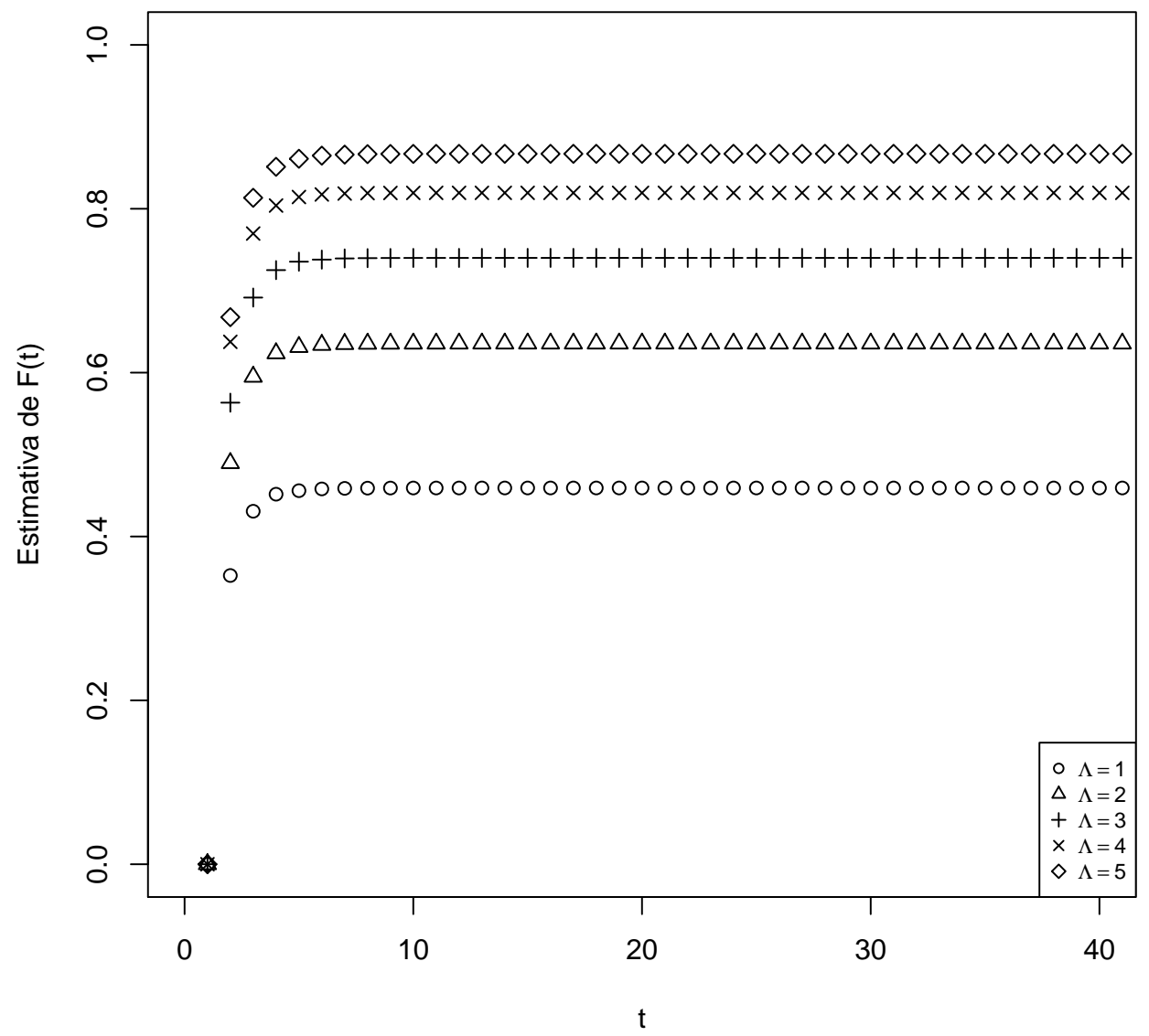

Figura 4.10: Estimativas de $F(t) \mid \tau=1$ variando de acordo com valores de $\Lambda$ 
$66 \boldsymbol{A} \boldsymbol{B} \boldsymbol{C}$ APLICADO AO MODELO BAYESIANO NÃO-PARAMÉTRICO PARA O MONITORAMENTO "ON-LINE" DE QUALIDADE DE TAGUCHI PARA ATRIBUTOS.

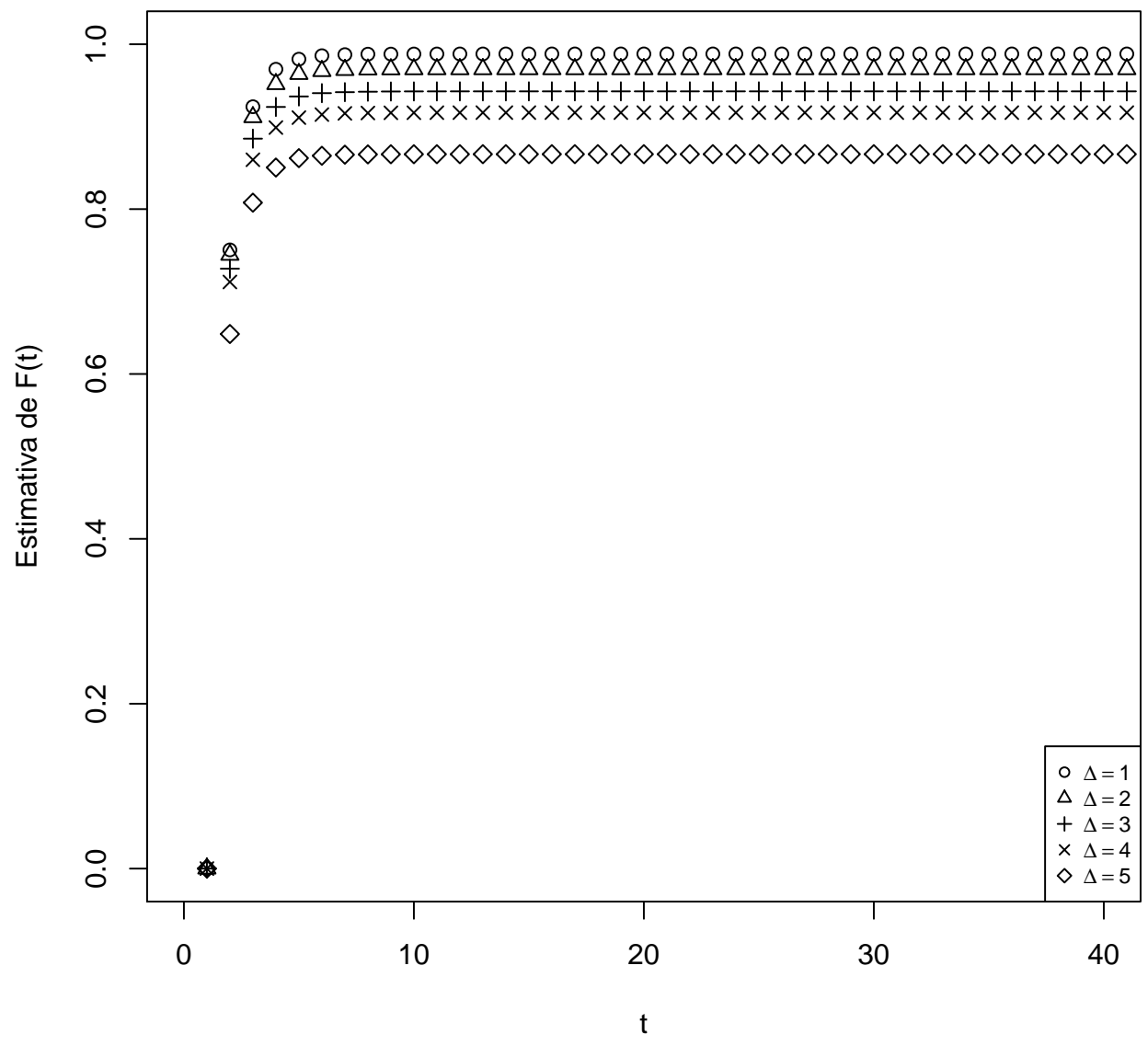

Figura 4.11: Estimativas de $F(t) \mid \tau=1$ variando de acordo com valores de $\Delta$ 


\section{Capítulo 5}

\section{$A B C$ aplicado a Enviromental Stress Screening}

\subsection{Introdução}

Uma outra ferramenta utilizada para aferir a qualidade de itens produzidos por um determinado processo será apresentada aqui.

O Enviromental Stress Screening, denominado a partir de agora como $\boldsymbol{E S S}$, é útil para eliminar, da população de itens produzidos, por um determinado processo, aqueles que possam possuir defeitos latentes. De certa forma pode-se dizer que o $\boldsymbol{E} \boldsymbol{S} \boldsymbol{S}$ é o ultimo estágio para a deteç̧ão da ausência do controle da qualidade do processo de produção.

A proposta do $\boldsymbol{E} \boldsymbol{S} \boldsymbol{S}$ é simular o desgaste que um item saído da linha de produção sofre devido a anos de uso, mas em um tempo reduzido.

O procedimento da investigação de estresse dá-se da seguinte forma:

1. Amostrar um lote de $N$ itens produzidos;

2. Submeter os itens produzidos ao estresse, de forma acelerada, de anos de uso convencional;

3. Apos o término do ensaio de estresse acelerado, investigam-se os itens que sobreviveram, ou não, ao estresse.

4. Dos não sobreviventes investiga-se se o item produzido é, ou não, de boa qualidade.

Neste capítulo será discutido um modelo estatístico, sob a linha de pensamento da inferência Bayesiana, apresentado em Barlow et al. (1994), para a metodologia ESS.

\subsection{Abordagem Bayesiana para a metodologia ESS}

Considere um lote de $N$ itens produzidos e, que dentre esses itens, existem itens de boa qualidade, e outros que parecem ser de boa qualidade, mas de fato não o são, pois possuem um defeito latente que não pôde ser detectado pelos métodos de aferição da qualidade realizados anteriormente no processo de produção.

No trabalho de Barlow et al. (1994) esse cenário é abordado. A utilização do $\boldsymbol{E} \boldsymbol{S} \boldsymbol{S}$ é proposta para detectar a qualidade dos itens produzidos. No trabalho em questão, foram feitas algumas suposições sobre a investigação de estresse, dentre elas, eles consideram que em um lote de $N$ itens existe uma proporção $p$ de itens de uma qualidade inferior (características latentes do item que não atendem determinadas especificações).

A taxa de falha, tempo até apresentar um mal funcionamento, dos itens de qualidade inferior é estabelecida como $\lambda_{e}^{\prime}$, enquanto a taxa de falha dos itens de boa qualidade é $\lambda_{g}^{\prime}$, onde $\lambda_{e}^{\prime}>\lambda_{g}^{\prime}$.

Como os ensaios de estresse simulam o estresse de toda uma vida de uso do item em um intervalo de tempo reduzido, Barlow et al. (1994) estipulam um fator de aceleração do ensaio, representado como $l$, e deste modo, $\lambda_{e}=l \lambda_{e}^{\prime}$ representa a taxa de falha do item de qualidade inferior submetido ao estresse de um ensaio, e $\lambda_{g}=l \lambda_{g}^{\prime}$ representa a taxa de falha do item de boa qualidade submetido ao estresse de um ensaio. A relação se mantêm a mesma, $\lambda_{e}>\lambda_{g}$.

Ainda sobre as taxas, Barlow et al. (1994) dão argumentos sobre a validade de supor que as taxas de falha $\lambda_{e}$ e $\lambda_{g}$ são constantes e, assim, assumir uma distribuição Exponencial para o tempo de vida de cada item, implicando diretamente que os itens não envelhecem com o tempo. 
Considere que um item, dentre os produzidos é selecionado aleatoriamente. Não é sabido se este item em questão é de boa ou baixa qualidade e, assim, seja $X$ o tempo de vida, sob o estresse acelerado, deste item, então:

$$
P\left(X>x \mid p, \lambda_{g}, \lambda_{e}\right)=p e^{-\lambda_{e} x}+(1-p) e^{-\lambda_{g} x} .
$$

Como $\left(p, \lambda_{g}, \lambda_{e}\right)$ não podem ser observados, serão tratados como parâmetros, quantidades desconhecidas. Deste modo, do ponto de vista Bayesiano, pode-se atribuir distribuições de probabilidade representando a opinião, de um especialista por exemplo, a priori. Barlow et al. (1994) sugerem o uso da família de distribuições Beta como densidade a priori para $p$. Assim $p \sim \operatorname{Beta}(a, b)$, onde $a, b>0$. Como há a relação entre as taxas de falhas $\left(\lambda_{e}>\lambda_{g}\right)$, também é sugerido, atribuir, de forma independente de $p$, uma distribuição Exponencial, com hiperparâmetro $\theta$, para $\lambda_{g}$ e uma distribuição Exponencial deslocada por $\lambda_{g}$, com hiperparâmetro $\tau$ para $\lambda_{e}, \operatorname{com} \tau, \theta>0$. De forma analítica:

$$
\pi\left(p, \lambda_{g}, \lambda_{e}\right)=\theta \tau \frac{\Gamma(a+b)}{\Gamma(a) \Gamma(b)} p^{a-1}(1-p)^{b-1} e^{-\lambda_{g}(\theta-\tau)} e^{-\lambda_{e} \tau} \mathbb{I}_{(0,1)}(p) \mathbb{I}_{(0, \infty)}\left(\lambda_{g}\right) \mathbb{I}_{\left(\lambda_{g}, \infty\right)}\left(\lambda_{e}\right) .
$$

A ação de coletar e estressar um lote de itens envolve certos custos. Barlow et al. (1994) estipulam duas classes de custos, uma ligada a decisões que podem ser tomadas, como o custo de um item de baixa qualidade sobreviver ao estresse (denominado $c_{1}$ ) e o custo de um item de boa qualidade ser destruído durante o estresse (denominado $c_{2}$ ). A outra classe de custos compreende custos de estresse. O custo de estressar e destruir um item e esse item ser destruído (denominado $c_{3}$ ) e o custo de estressar um item e esse item ser liberado (denominado $c_{4}$ ).

Seja $T$ o tempo ótimo da duração do estresse aplicado aos itens a serem investigados (com um determinado nível de estresse $l$ ), e seja $t$ o tempo de duração do estresse. A função de perda que envolve todos os custos já citados, envolvendo $l$ e $t$, depende de $p, \lambda_{g}$ e $\lambda_{e}$. Assim, o custo condicional por peça, em uma investigação de estresse com fator de estresse $l$ e duração $t$, utilizando a equação 5.1 como base, é dado pela seguinte forma,

$$
p\left[\left(1-e^{-\lambda_{e} t}\right) c_{3}+e^{-\lambda_{e} t}\left(c_{1}+c_{4}\right)\right]+(1-p)\left[\left(1-e^{-\lambda_{g} t}\right)\left(c_{2}+c_{3}\right)+e^{-\lambda_{g} t} c_{4}\right] .
$$

\subsubsection{Função de risco a priori}

O passo seguinte, numa análise estatística, seria construir a função de risco, a priori, do modelo. Essa função nada mais é a expectativa, a priori, dos custos de estressar um item. Barlow et al. (1994) estabelece a função de risco para a investigação por estresse, com a finalidade de determinar o tempo ótimo, $T$, de duração da investigação de estresse dos itens. Tempo este que minimiza a expectativa dos custos envolvidos no ensaio de estresse.

Pensando nisso Barlow et al. (1994) desenvolvem a expressão 5.3, simplificando-a, obtendo a seguinte expressão:

$$
c_{3}+c_{2}(1-p)+\left(c_{1}+c_{4}-c_{3}\right) p e^{-\lambda_{e} t}+\left(c_{4}-c_{2}-c_{3}\right)(1-p) e^{-\lambda_{g} t} .
$$

Notem que a influência do fator de estresse, $l$, na função de custo está representado pelas taxas de falhas (lembrando que $\lambda_{g}=l \lambda_{g}^{\prime}$ e $\lambda_{e}=l \lambda_{e}^{\prime}$ ). Com isso, a expressão da função de risco, a priori, é a seguinte:

$$
R(t, l)=c_{3}+c_{2} \frac{b}{a+b}+\left(c_{1}+c_{4}-c_{3}\right) \frac{a}{a+b} \frac{\theta \tau}{(\tau+t)(\theta+t)}+\left(c_{4}-c_{2}-c_{3}\right) \frac{b}{a+b} \frac{\theta}{\theta+t} .
$$

\subsubsection{Análise Bayesiana}

Após estabelecer todas as suposições a priori sobre o tempo de vida de um item produzido, e dos custos envolvidos em submeter este item ao estresse acelerado, é hora de fazer as suposições sobre os dados que serão observados ao final da investigação por estresse. 
Como descrito no início deste capítulo, a metodologia $\boldsymbol{E S S}$ consiste em submeter um lote, de tamanho $N$, de itens a um estresse de uso, estresse esse mais intenso que o usual. Após um tempo $t$ observa-se a proporção de itens que falharam durante a duração do estresse. Os itens que falharam submetemos a uma autopsia e, então, verifica-se a a qualidade desse item, se é ou não de boa qualidade. Também há itens que não sobreviveram ao estresse dos quais não foi possível extrair informação sobre sua qualidade com a realização da autopsia. Barlow et al. (1994) consideram então:

- $x$ : número de itens que falharam e são de baixa qualidade;

- y: número de itens que falharam e são de boa qualidade;

- z: número de itens que falharam e que não é sabido se são de boa ou baixa qualidade;

- $m=N-x-y-z$ : número de itens que sobreviveram ao estresse.

Com essas considerações, Barlow et al. (1994) definem a função de verossimilhança dos dados da seguinte forma:

$$
\begin{aligned}
L\left(\alpha, p, \lambda_{g}, \lambda_{e} \mid x, y, z, m\right) & =\left[p\left(1-e^{-\lambda_{e} T}\right)(1-\alpha)\right]^{x} \times\left[(1-p)\left(1-e^{-\lambda_{g} T}\right)(1-\alpha)\right]^{y} \\
& \times\left[\alpha p\left(1-e^{-\lambda_{e} T}\right)+\alpha(1-p)\left(1-e^{-\lambda_{g} T}\right)\right]^{z} \\
& \times\left[p e^{-\lambda_{e} T}+(1-p) e^{-\lambda_{g} T}\right]^{m}
\end{aligned}
$$

Nessa expressão é introduzida uma incógnita, $\alpha$. Esse novo elemento no modelo foi utilizado para representar a probabilidade da autópsia conseguir, ou não, qualificar a qualidade de um item que não sobreviveu ao estresse. Em outras palavras $\alpha$ e sua distribuição a priori (denominada $h(\alpha)$ ) representam a opinião a priori sobre o quão eficiente é o processo de autopsia utilizado. Supõe-se que a distribuição a priori de $\alpha$ seja independente de $\left(p, \lambda_{g}, \lambda\right)$, pois não há razões para crer que o processo de autópsia sofra algum tipo interferência da proporção de itens de baixa qualiade no lote, ou das taxas de falha dos itens, independente da qualidade.

Aplicando o teorema de Bayes, tem-se a expressão da distribuição conjunta a posteriori de $\left(\alpha, p, \lambda_{g}, \lambda_{e}\right)$,

$$
\pi\left(\alpha, p, \lambda_{g}, \lambda \mid x, y, z, m\right)=\mathbf{C} h(\alpha) \pi\left(p, \lambda_{g}, \lambda_{e}\right) L\left(\alpha, p, \lambda_{g}, \lambda_{e} \mid x, y, z, m\right),
$$

onde $\mathbf{C}$ é a constante de normalização da densidade.

Fazendo todas as manipulações algébricas necessárias, tem-se:

$$
\begin{aligned}
& \pi\left(\alpha, p, \lambda_{g}, \lambda_{e} \mid x, y, z, m\right)=\mathbf{C}\left[h(\alpha) \alpha^{z}(1-\alpha)^{x+y}\right] \\
\times & p^{a+x-1}(1-p)^{b+y-1} \sum_{i=0}^{x} \sum_{j=0}^{y} \sum_{l=0}^{m} \sum_{k=0}^{z} \sum_{r=0}^{k} \sum_{s=0}^{z-k}\left(\begin{array}{c}
x \\
i
\end{array}\right)\left(\begin{array}{c}
y \\
j
\end{array}\right)\left(\begin{array}{c}
m \\
l
\end{array}\right)\left(\begin{array}{c}
z \\
k
\end{array}\right)\left(\begin{array}{c}
k \\
r
\end{array}\right)\left(\begin{array}{c}
z-k \\
s
\end{array}\right) \\
\times \quad & (-1)^{x+y+z-i-j-r-s} \times p^{k+l}(1-p)^{m-l+z-k} e^{-\lambda_{e} T(x-i+l+k-r+\tau / T)} \\
\times & e^{-\lambda_{g} T(y-j+m-l+z-k-s+(\theta-\tau) / T)} .
\end{aligned}
$$

De maneira similar ao problema encontrado no capítulo 4, realizar cálculos de probabilidade na função de probabilidade a posteriori conjunta de $\left(\alpha, p, \lambda_{g}, \lambda_{e}\right)$ é uma tarefa bastante complexa. Quanto maior o tamanho do lote de itens, mais complicado se torna efetuar qualquer tipo de cálculo de probabilidade. A principal dificuldade é a presença de vários somatórios. É fácil ver que o número total de parcelas calculadas, que depende essencialmente dos valores de $x, y, z$ e $m$. O número total de parcelas a serem somadas na equação 5.8 é da forma:

$$
n p=(x+1)(y+1)(z+1)(m+1)\left[2^{z}+z(z+1)\right],
$$

onde $n p$ é o número de parcelas. 
Qualquer manipulação, ou cálculo que deseje fazer na equação 5.8, seja para um simples cálculo de esperança, encontrando assim a estimativa de Bayes sob perda quadrática, ou para tarefas mais complexas como minimizar uma função de risco, a posteriori, encontra uma grande barreira na forma analitica da distribuição a posteriori de $\left(\alpha, p, \lambda_{g}, \lambda_{e}\right)$.

Para qualquer tarefa que envolva a manipulação da equação 5.8 métodos numéricos são imprescindíveis. Como a função de verossimilhança tem uma forma analítica bastante complicada, o $A B C$ é uma boa indicação para resolver os problemas aqui apresentados.

\subsection{Usando o $A B C$ na metodologia $E S S$}

Como já foi exaustivamente comentado nos capítulos anteriores, para utilizar um algoritmo $\boldsymbol{A} \boldsymbol{B} \boldsymbol{C}$ é necessário saber como gerar observações da distribuição a priori, e saber como replicar o experimento observado.

Não houve preocupações relativas a suposições de distribuições a priori pelo fato delas serem bem definidas em Barlow et al. (1994), apenas aqui foi definido uma distribuição uniforme no intervalo $[0,1]$ como distribuição priori para $\alpha$.

A primeira preocupação, real, ao configurar o $\boldsymbol{A} \boldsymbol{B} \boldsymbol{C}$ para ser útil a uma análise estatística que utiliza-se da estratégia $\boldsymbol{E S S}$, é em como replicar o experimento. Felizmente Barlow et al. (1994) apresentam um diagrama de influência que, possibilita suplantar esta preocupação.

Ironicamente, a própria função de verossimilhança auxilia a como replicar o experimento. Os termos entre colchetes da equação 5.6 podem ser utilizados como probabilidades de um determinado item testado ser classificado em uma determinada categoria. Deste modo o item vai estar na categoria 1 com probabilidade $p\left(1-e^{-\lambda_{e} T}\right)(1-\alpha)$, na categoria 2 com probabilidade $(1-p)\left(1-e^{-\lambda_{g} T}\right)(1-\alpha)$, categoria 3 com probabilidade $\alpha p\left(1-e^{-\lambda_{e} T}\right)+\alpha(1-p)\left(1-e^{-\lambda_{g} T}\right)$ e, finalmente, o item é classificado na categoria $4 \mathrm{com}$ probabilidade $p e^{-\lambda_{e} T}+(1-p) e^{-\lambda_{g} T}$. Note que o somatório das quatro probabilidades é igual a 1, caracterizando uma distribuição Multinomial com os parâmetros $\boldsymbol{\Theta}=\left[p\left(1-e^{-\lambda_{e} T}\right)(1-\alpha),(1-p)\left(1-e^{-\lambda_{g} T}\right)(1-\alpha), p\left(1-e^{-\lambda_{e} T}\right)+\alpha(1-p)(1-\right.$ $\left.\left.e^{-\lambda_{g} T}\right), p e^{-\lambda_{e} T}+(1-p) e^{-\lambda_{g} T}\right]$ e $N$ para o vetor $\mathbf{x}=(x, y, z, m)$.

As quatro categorias citadas acima referem-se ao que pode acontecer com um item submetido a estresse: sobreviver, não sobreviver e ser de baixa qualidade, não sobreviver e ser de boa qualidade e não sobreviver e não conseguir atestar sua qualidade.

Como o vetor de observações é um vetor de componentes discretas, então escolheu-se o $\boldsymbol{A} \boldsymbol{B C}$ Rejeição 1, algoritmo 1 para gerar observações da distribuição a posteriori de $\left(\alpha, p, \lambda_{g}, \lambda_{e}\right)$. Abaixo é apresentado o algoritmo $\boldsymbol{A} \boldsymbol{B C}$ já configurado para gerar amostras da densidade a posteriori, equação 5.8. O vetor observado é representado por $\mathbf{x}$

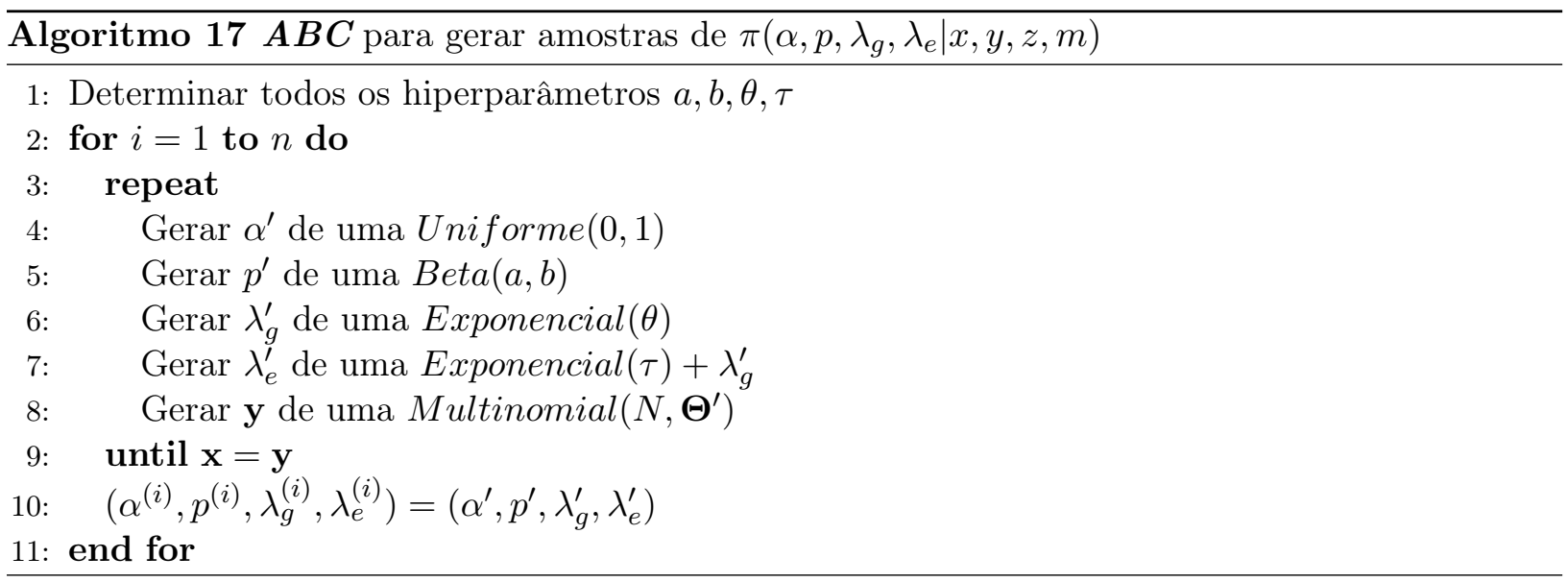

\subsubsection{Exemplo numérico}

Para ilustrar o funcionamento do $\boldsymbol{A B C}$ gerando amostras da função densidade 5.8 , foi considerada uma situação hipotética. Suponha que um certo lote de itens será colocado sob estresse, e um especialista do processo de produção dos itens dá a informação sobre o processo de produção, e do 
tempo de vida dos itens de baixa e alta qualidade. Com essas informações, é possível estabelecer as seguintes distribuições a priori:

$$
\begin{aligned}
p & \sim \operatorname{Beta}(1,2) ; \\
\lambda_{g} & \sim \operatorname{Exponencial}(2) ; \\
\lambda_{e} & \sim \lambda_{g}+\operatorname{Exponencial}(3) ; \\
\alpha & \sim \operatorname{Uniforme}(0,1) .
\end{aligned}
$$

Com as distribuições a priori bem definidas, é interessante encontrar um valor ótimo para $T$ usando a função de perda 5.3. Os custos $c_{1}, c_{2}, c_{3}, c_{4}$ utilizados foram os mesmos utilizados em Barlow et al. (1994, seção 4) $\left(c_{1}=100, c_{2}=20, c_{3}=1, c_{4}=0,01\right)$. O tempo ótimo a priori, obtido otimizando a função de perda a priori, é $T=10,63$ horas, que gera um custo esperado, por item submetido ao estresse de 15,56.

O departamento de controle de qualidade decidiu então submeter um lote de 1000 itens a um estresse com o objetivo de estimar a proporção de itens de baixa qualidade, o que gera um custo total de 15560. Dos 1000 itens, 167 itens não sobreviveram ao estresse e foram diagnosticados pela autopsia como de baixa qualidade, 332 itens que não sobreviveram ao estresse e foram diagnosticados pela autopsia como de boa qualidade, 498 itens não sobreviveram ao estresse e o processo de autopsia não conseguiu concluir sobre a qualidade destes itens e, apenas 3 itens sobreviveram ao estresse.

A distribuição a posteriori, depois de observar o resultado do ensaio de estresse, é da seguinte forma:

$$
\begin{aligned}
& \pi\left(\alpha, p, \lambda_{g}, \lambda_{e} \mid x, y, z, m\right)=\mathbf{C}\left[\alpha^{498}(1-\alpha)^{499}\right] \\
\times & p^{167}(1-p)^{333} \sum_{i=0}^{167} \sum_{j=0}^{332} \sum_{l=0}^{3} \sum_{k=0}^{498} \sum_{r=0}^{k} \sum_{s=0}^{498-k}\left(\begin{array}{c}
167 \\
i
\end{array}\right)\left(\begin{array}{c}
332 \\
j
\end{array}\right)\left(\begin{array}{c}
3 \\
l
\end{array}\right)\left(\begin{array}{c}
498 \\
k
\end{array}\right)\left(\begin{array}{c}
k \\
r
\end{array}\right)\left(\begin{array}{c}
498-k \\
s
\end{array}\right) \\
\times & (-1)^{997-i-j-r-s} \times p^{k+1}(1-p)^{501-l-k} e^{-\lambda_{e}(10,63)(167,2822-i+l+k-r)} \\
\times & e^{-\lambda_{g}(10,63)(667,9059-j-l-k-s)}
\end{aligned}
$$

A densidade a posteriori desse exemplo, como já era esperado, é difícil de ser manipulada e, também difícil de ser aproximada pelo $\boldsymbol{A B C}$, algoritmo 17. Em uma distribuição Multinomial, com um espaço amostral tão vasto quanto o desse exemplo, qual a probabilidade de observar a amostra que foi observada $(\mathbf{x}=(167,332,498,3))$ ?. No melhor dos casos, quando o vetor de probabilidades é igual o vetor de proporções amostrais, isto é, $\Theta=(0,498,0,332,0,167,0,003)$, $P(\mathbf{X}=(167,332,498,3) \mid \Theta=(0,498,0,332,0,167,0,003))=0,00021441$. Essa baixa probabilidade implica diretamente no desempenho do $\boldsymbol{A B C}$, tornando a taxa de rejeição muito alta. Visando diminuir a taxa de rejeição rejeição o algoritmo 17 foi modificado, introduzindo os três argumentos computacionais, tunning parameters, presentes no algoritmo 2 e comentados na seção 2.3 , resultando no algoritmo 18.

A distância euclidiana foi escolhida como $\rho(\cdot)$. Definiu-se $\epsilon=10^{-2}$ e, como sumário de amostra utilizou-se a própria amostra dividida por $N$, vetor de proporções amostrais $(\eta(\mathbf{x})=\mathbf{x} / N)$.

As amostras geradas pelo algoritmo 18 estão apresentadas, na forma de um histograma, na figura 5.1. Vê-se amostras coerentes com o que era esperado. As distribuições a priori de $\alpha$ e $p$ são da família de distribuições Beta, assim como parece ser sua distribuição a posteriori. As distribuições a priori de $\lambda_{g}$ e $\lambda_{e}$ são da família de distribuições Gama, deslocadas, mas ainda Gama. De acordo com os histogramas as distribuições a posteriori de $\lambda_{g}$ e $\lambda_{e}$ parecem ser distribuições Gama e deslocadas também. A estimativa de Bayes, com relação a perda quadrática, para $\left(p, \lambda_{g}, \lambda_{e}\right)$ é $(0,3312,0,8655,1,1860)$. Na figura 5.2 tem-se a função de risco a posteriori avaliada em vários tempos $t$. Calculou-se o risco esperado de forma numérica utilizando as amostras geradas. Minimizando a função de perda, verifica-se que o tempo ótimo para a duração do estresse, a posteriori, é 10,01 horas. 


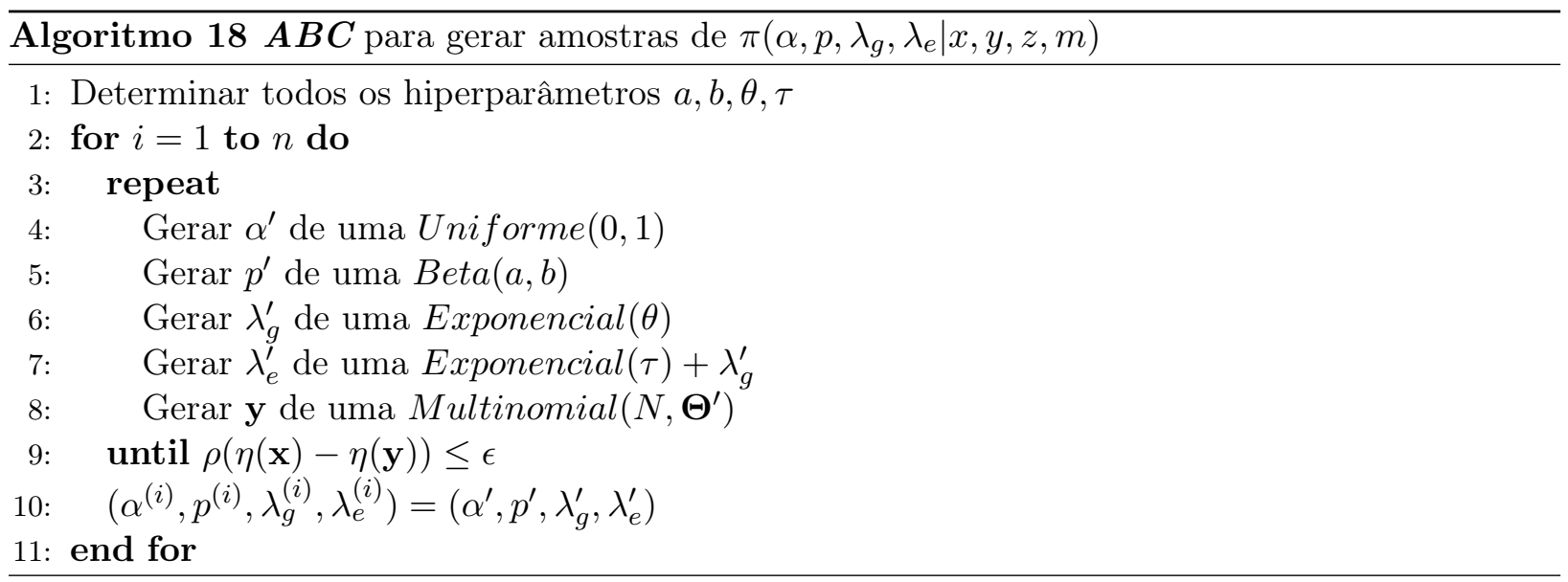

\subsection{MCMC aplicado à metodologia $E S S$}

Com a intenção de comparar os desempenhos do $\boldsymbol{A B C}$ com o de um algoritmo $\boldsymbol{M C M C}$, mais precisamente um algoritmo Metropolis-Hastings, o mesmo será configurado para gerar observações do modelo 5.8 .

Como abordado no capítulo 3, para construir um algoritmo Metropolis-Hastings é necessário uma função que informa a probabilidade da cadeia de Markov em questão ir de um estado a outro, kernel de transição, $q(\theta, \phi)$, e da probabilidade de aceitação, $\alpha(\theta, \phi)$. Foi escolhido o método Independece chain. Tal escolha foi motivada pelo fato de, em sua metodologia, não haver necessidade de introduzir um parâmetro de tunning para controlar a variância do kernel de transição e a probabilidade de rejeição do algoritmo.

Como kernel de transição decidui-se por usar a função densidade conjunta a priori de $\left(\alpha, p, \lambda_{g}, \lambda_{e}\right)$. Como definido anteriormente, $\alpha \sim \operatorname{Uniforme}(0,1)$, e portanto:

$$
q\left(\Theta^{(j-1)}, \Theta^{\prime}\right)=\theta \tau \frac{\Gamma(a+b)}{\Gamma(a) \Gamma(b)} p^{\prime a-1}\left(1-p^{\prime}\right)^{b-1} e^{-\lambda_{g}^{\prime}(\theta-\tau)} e^{-\lambda_{e}^{\prime} \tau} \mathbb{I}_{(0,1)}\left(\alpha^{\prime}\right) \mathbb{I}_{(0,1)}\left(p^{\prime}\right) \mathbb{I}_{(0, \infty)}\left(\lambda_{g}^{\prime}\right) \mathbb{I}_{\left(\lambda_{g}^{\prime}, \infty\right)}\left(\lambda_{e}^{\prime}\right),
$$

onde, $\Theta^{(j-1)}=\left(\alpha^{(j-1)}, p^{(j-1)}, \lambda_{g}^{(j-1)}, \lambda_{e}^{(j-1)}\right)$ são os estados da cadeia no tempo $j-1$ e, $\Theta^{\prime}=$ $\left(\alpha^{\prime}, p^{\prime}, \lambda_{g}^{\prime}, \lambda_{e}^{\prime}\right)$ são os valores gerados pelo kernel de transição que serão testados e, caso aceitos, serão os valores da cadeia no tempo $j$. Deste modo a razão de teste é,

$$
\begin{aligned}
\alpha\left(\Theta^{(j-1)}, \Theta^{\prime}\right) & =\min \left\{1, \frac{\pi\left(\Theta^{\prime} \mid(x, y, z, m)\right) q\left(\Theta^{\prime}, \Theta^{(j-1)}\right)}{\pi\left(\Theta^{(j-1)} \mid(x, y, z, m)\right) q\left(\Theta^{(j-1)}, \Theta^{\prime}\right)}\right\} \\
& =\min \left\{1, \frac{\pi\left(\Theta^{\prime} \mid(x, y, z, m)\right) p^{(j-1)^{a-1}}\left(1-p^{(j-1)}\right)^{b-1} e^{-\lambda_{g}^{(j-1)}(\theta-\tau)} e^{-\lambda_{e}^{(j-1)} \tau}}{\pi\left(\Theta^{(j-1)} \mid(x, y, z, m)\right) p^{\prime a-1}\left(1-p^{\prime}\right)^{b-1} e^{-\lambda_{g}^{\prime}(\theta-\tau)} e^{-\lambda_{e}^{\prime} \tau}}\right\} .
\end{aligned}
$$

Onde, $\pi(\cdot \mid(x, y, z, m))$ é a densidade a posteriori do modelo de Barlow et al. (1994), equação 5.8.

Note que, pelo menos uma vez a cada iteração do algoritmo, será necessário calcular $\pi(\cdot \mid(x, y, z, m))$ para um determinado ponto. Deste modo será necessário calcular, e depois somar, todas as parcelas de 5.8, que dependerão dos valores de $(x, y, m, z)$. Considerando o exemplo da seção 5.3.1, $(x, y, z, m)=(167,332,498,3)$, onde um lote de 1000 itens que foi submetido a um ensaio de estresse acelerado. De acordo com a equação 5.9 , só o fator $(x+1)(y+1)(z+1)(m+1)$ resulta em 111, 664, 224 parcelas. Como a variável $z$ observada é relativamente grande, tem-se 499 combinações diferentes de limites superiores para os somatórios mais internos da densidade 5.8. Ao considerar as parcelas destes somatórios internos, para cada limite superior diferente, obtêm-se um número muito grande, $9,1380 \times 10^{157}$ parcelas.

Um número tão grande de parcelas, inviabiliza a utilização de qualquer algoritmo amostrador que necessite efetuar cálculos na função de verossimilhança, dentre eles o $\boldsymbol{M C M C}$. 
Como o objetivo desta seção em si é apenas ilustrar o funcionamento de um $\boldsymbol{M C M C}$ para gerar observações do modelo 5.8 não será utilizada a mesma amostra do exemplo numérico anterior, mas sim uma nova amostra tal que o número de itens submetidos à metodologia $\boldsymbol{E} \boldsymbol{S} \boldsymbol{S}$ é menor.

Considere uma situação hipotética em que num lote de 10 itens submetidos a estresse, 2 itens falharam sob estresse e são comprovadamente de baixa qualidade; 3 itens falharam e são comprovadamente de boa qualidade; 1 item falhou sob as condições de estresse e não se sabe sua qualidade; 4 itens sobreviveram ao teste de estresse. Com essa amostra o número total de parcelas da densidade a posteriori é de 240 .

O pseudo-código do algoritmo $\boldsymbol{M C M C}$ configurado para o modelo em estudo, encontra-se abaixo, algoritmo 19. Assim como as outras amostras geradas com um algoritmo $\boldsymbol{M C M C}$ dos capítulos anteriores, decidiu-se, como regra para diagnosticar a convergência da cadeia, um critério gráfico. Quando a média ergódica da cadeia se mantém igual, com quatro casas decimais, por 500 iterações, então a cadeia convergiu.

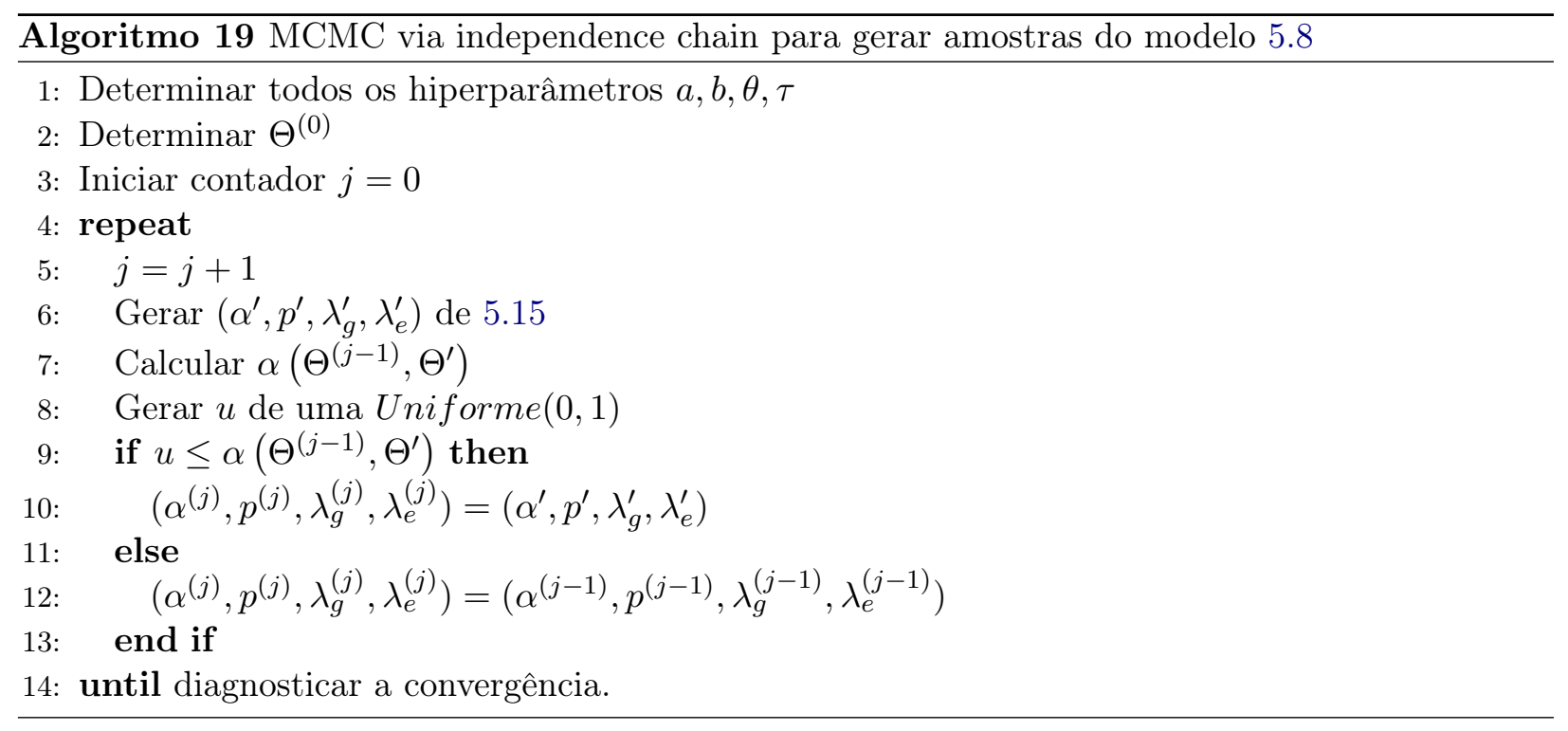

Para evitar problemas com a dependência, indesejada, dentro da amostra, foi decidido que, para a geração de cada unidade amostral uma nova cadeia seria iniciada, com um novo estado inicial, produzindo assim uma amostra com observações independentes.

Os histogramas das amostras geradas pelo algoritmo 19 estão apresentados na figura 5.3. Percebe-se que $\alpha$ e $p$ apresentam um comportamento semelhante a distribuições Betas, o que era esperado, já que suas distribuições a priori são desta família e as condições do experimento de estresse não dão motivos para que haja uma mudança drástica nas crenças a priori.

Os histogramas de $\lambda_{g}$ e $\lambda_{e}$ também se comportam como esperado, mantendo um comportamento similar a uma distribuição Gama e mantendo a relação de dependência, estipulada em Barlow et al. (1994), $\lambda_{e}>\lambda_{g}$.

No âmbito da qualidade de amostras geradas, o $\boldsymbol{M C M C}$ se saiu tão bem quanto o $\boldsymbol{A B C}$, os histogramas das amostras geradas pelos dois métodos ficaram dentro do esperado. Como não há meios de saber a forma, gráfica, que a distribuição a posteriori possui, não se pode atestar o quão próximas as distribuições geradas com o $\boldsymbol{A B C}$ e о $\boldsymbol{M C M C}$ estão da distribuição alvo. No âmbito da eficiência computacional o $\boldsymbol{A} \boldsymbol{B} \boldsymbol{C}$ demonstra-se mais eficiente, pois para gerar uma unidade amostral o $\boldsymbol{M C M C}$ levou, em média, 27206 iterações, enquanto o $\boldsymbol{A} \boldsymbol{B} \boldsymbol{C}$ demorou apenas 6780 interações, em média.

\subsection{Taxa de fala crescente}

Uma outra situação, bastante plausível num mundo prático, é quando os itens produzidos envelhecem com o tempo e, assim, quanto mais antigo o item é mais fácil esse item apresentar uma falha. Essa situação é bastante comum em peças automotivas, amortecedores, discos de freios, entre 
outras, que necessitam ser trocados de tempos em tempo, ou de acordo com o uso e desgaste da peça.

Do ponto de vista estatístico, sob a ótica de análise de sobrevivência, essa nova situação em estudo impede a suposição de uma taxa de falha constante para os itens produzidos e, por consequência, não é possível usar a equação 5.21 como função de sobrevivência de um item produzido.

Para resolver esse problema, é sugerido usar a distribuição Weibull como a distribuição de probabilidade do tempo de vida de um item produzido. Para relembrar, a função densidade da distribuição Weibull é da forma:

$$
f(x \mid k, \lambda)=\frac{k}{\lambda}\left(\frac{x}{\lambda}\right)^{k-1} e^{-\left(\frac{x}{\lambda}\right)^{k}}
$$

E a taxa de falha é da forma:

$$
h(x \mid k, \lambda)=\frac{k}{\lambda}\left(\frac{x}{\lambda}\right)^{k-1}
$$

com $x, \lambda>0$. Se $k=1$ tem-se uma taxa de falha constante, coincidindo com a taxa de falha da distribuição Exponencial, já quando $k<1$ a taxa de falha é decrescente, e quando $k>1$ ela é crescente, caso abordado aqui.

A escolha da distribuição Weibull foi motivada pelo fato da taxa de falha gerada por essa distribuição ter uma forma analítica fácil de ser manipulada.

\subsubsection{Modelo com taxa de falha crescente}

Com essa alteração de suposições, quais as consequências no modelo apresentado em Barlow et al. (1994)? A seguir são apresentadas as modificações nesse modelo.

Seja $h_{g}(x \mid k, \lambda)$ a taxa de falha dos itens de boa qualidade e, seja $h_{e}(x \mid k, \lambda)$ a taxa de falha dos itens de baixa qualidade. Sejam também $E_{g}(x)$ o tempo esperado até a falha do item de boa qualidade e $E_{e}(x)$ o tempo esperado até a falha do item de baixa qualidade. É desejável que $h_{g}(x \mid k, \lambda)<h_{e}(x \mid k, \lambda)$ e $E_{g}(x)>E_{e}(x)$. Dessas duas condições é possível afirmar que:

$$
\begin{aligned}
\lambda_{g} & >\lambda_{e} \\
1<k_{g} & <k_{e} .
\end{aligned}
$$

Como a distribuição de probabilidade para o tempo de vida dos itens produzidos é modificada, a função de sobrevivência também deve ser mudada. Assim,

$$
P\left(X>x \mid p, \lambda_{g}, \lambda_{e}, k_{g}, k_{e}\right)=p e^{-\left(\frac{x}{\lambda_{e}}\right)^{k_{e}}}+(1-p) e^{-\left(\frac{x}{\lambda_{g}}\right)^{k_{g}}} .
$$

As suposições e significados dos custos envolvidos no $\boldsymbol{E S S}$ mantiveram-se inalteradas. Portanto a única modificação foi na função de perda (equação 5.3), e é motivada pela troca da distribuição do tempo de vida dos itens produzidos, exponencial por Weibull. Assim, a nova função de perda é da seguinte forma:

$l\left(p, \lambda_{g}, \lambda_{e}, k_{g}, k_{e}\right)=p\left[\left(1-e^{-\left(\frac{t}{\lambda_{e}}\right)^{k_{e}}}\right) c_{3}+e^{-\left(\frac{t}{\lambda_{e}}\right)^{k_{e}}}\left(c_{1}+c_{4}\right)\right]+(1-p)\left[\left(1-e^{-\left(\frac{t}{\lambda_{g}}\right)^{k_{g}}}\right)\left(c_{2}+c_{3}\right)+e^{-\left(\frac{t}{\lambda_{g}}\right)^{k_{g}}} c\right.$

Os argumentos dados por Barlow et al. (1994) para justificar a escolha de uma família de distribuições Beta para $p$ e distribuições Exponenciais para $\lambda_{g}$ e $\lambda_{e}$ continuam valendo nesse novo caso. modificando apenasa relação entre $\lambda_{g}$ e $\lambda_{e}$ que foi invertida. Para $k_{g}$ e $k_{e}$, que são maiores que 1(equação 5.20), foram escolhidas, também, distribuições Exponenciais como prioris de $k_{g}$ e $k_{e}$ por ter uma forma analítica simples e de fácil manipulação. Deste modo, determinam-se as seguintes prioris para $p, \lambda_{g}, \lambda_{e}, k_{g}$ e $k_{e}$ :

$$
\begin{aligned}
p & \sim \operatorname{Beta}(a, b) \\
\lambda_{e} & \sim \operatorname{Exponencial}(\tau)
\end{aligned}
$$




$$
\begin{aligned}
\lambda_{g} & \sim \operatorname{Exponencial}(\theta)+\lambda_{e} \\
k_{g} & \sim \operatorname{Exponencial}(\rho)+1 ; \\
k_{e} & \sim \operatorname{Exponencial}(\varphi)+k_{g} .
\end{aligned}
$$

Com base das distribuições a priori marginais escreve-se a distribuição a priori conjunta de $\left(p, \lambda_{g}, \lambda_{e}, k_{g}, k_{e}\right)$ da seguinte maneira:

$$
\begin{aligned}
\pi\left(p, \lambda_{g}, \lambda_{e}, k_{g}, k_{e}\right) & =\tau \theta \varphi \rho e^{-\rho} \frac{\Gamma(a+b)}{\Gamma(a) \Gamma(b)} p^{a-1}(1-p)^{b-1} e^{-\lambda_{g} \theta} e^{-\lambda_{e}(\tau+\theta)} e^{-k_{e} \varphi} e^{-k_{g}(\rho-\varphi)} \times(5 . \\
& \times \mathbb{I}_{(0,1)}(p) \mathbb{I}_{(0, \infty)}\left(\lambda_{e}\right) \mathbb{I}_{\left(\lambda_{e}, \infty\right)}\left(\lambda_{g}\right) \mathbb{I}_{(1, \infty)}\left(k_{g}\right) \mathbb{I}_{\left(k_{g}, \infty\right)}\left(k_{e}\right)
\end{aligned}
$$

Infelizmente as prioris impostas aqui não possibilitam obter uma forma analítica fechada para a função de risco, $R(t, l)=E\left(l\left(p, \lambda_{g}, \lambda_{e}, k_{g}, k_{e}\right)\right)$, como na equação 5.5 e, para obter um tempo ótimo, $T$, é necessário usar métodos numéricos para tal tarefa. Simular $\left(p, \lambda_{g}, \lambda_{e}, k_{g}, k_{e}\right)$ a priori e calcular a função 5.22, e depois calcular a média, para cada valor de $t$ possibilitará a obtenção da duração ótima das investigações por estresse.

A verossimilhança, para quando os itens envelhecem, pouco se modifica, já que à função de sobrevivência da distribuição Weibull é muito semelhante a função de sobrevivência da distribuição exponencial. Portanto, a verossimilhança do problema é da forma:

$$
\begin{aligned}
L\left(\alpha, p, \lambda_{g}, \lambda_{e}, k_{g}, k_{e} \mid x, y, z, m\right) & =\left[p\left(1-e^{-\left(\frac{T}{\lambda_{e}}\right)^{k_{e}}}\right)(1-\alpha)\right]^{x} \\
& \times\left[(1-p)\left(1-e^{\left.-\left(\frac{T}{\lambda_{g}}\right)^{k_{g}}\right)}\right)(1-\alpha)\right]^{y} \\
& \times\left[\alpha p\left(1-e^{-\left(\frac{T}{\lambda_{e}}\right)^{k_{e}}}\right)+\alpha(1-p)\left(1-e^{\left.-\left(\frac{T}{\lambda_{g}}\right)^{k_{g}}\right)}\right]^{z}\right. \\
& \times\left[p e^{-\left(\frac{T}{\lambda_{e}}\right)^{k_{e}}}+(1-p) e^{-\left(\frac{T}{\lambda_{g}}\right)^{k_{g}}}\right]^{m} .
\end{aligned}
$$

O parâmetro $\alpha$ continua com as mesmas interpretações e suposição de distribuição a priori $(\alpha \sim$ Uniforme $(0,1))$.

Naturalmente, a distribuição de probabilidade a posteriori de $\left(\alpha, p, \lambda_{g}, \lambda_{e}, k_{g}, k_{e}\right)$ é da seguinte forma:

$$
\begin{aligned}
& \pi\left(\alpha, p, \lambda_{g}, \lambda_{e}, k_{g}, k_{e} \mid x, y, z, m\right) \propto \tau \theta \varphi \rho e^{-\rho} \frac{\Gamma(a+b)}{\Gamma(a) \Gamma(b)} p^{a-1}(1-p)^{b-1} e^{-\lambda_{g} \theta} e^{-\lambda_{e}(\tau+\theta)} e^{-k_{e} \varphi} e^{-k_{g}(\rho-\varphi)} \\
& \times\left[p\left(1-e^{-\left(\frac{T}{\lambda_{e}}\right)^{k_{e}}}\right)(1-\alpha)\right]^{x} \\
& \times\left[(1-p)\left(1-e^{-\left(\frac{T}{\lambda_{g}}\right)^{k_{g}}}\right)(1-\alpha)\right]^{y} \\
& \times\left[\alpha p\left(1-e^{-\left(\frac{T}{\lambda_{e}}\right)^{k_{e}}}\right)+\alpha(1-p)\left(1-e^{-\left(\frac{T}{\lambda_{g}}\right)^{k_{g}}}\right)\right]^{z} \\
& \times\left[p e^{-\left(\frac{T}{\lambda_{e}}\right)^{k_{e}}}+(1-p) e^{-\left(\frac{T}{\lambda_{g}}\right)^{k_{g}}}\right]^{m} \\
& \times \mathbb{I}_{(0,1)}(p) \mathbb{I}_{(0, \infty)}\left(\lambda_{e}\right) \mathbb{I}_{\left(\lambda_{e}, \infty\right)}\left(\lambda_{g}\right) \mathbb{I}_{(1, \infty)}\left(k_{g}\right) \mathbb{I}_{\left(k_{g}, \infty\right)}\left(k_{e}\right)
\end{aligned}
$$

e, de forma análoga à equação 5.8 , a distribuição de probabilidade a posteriori de $\left(\alpha, p, \lambda_{g}, \lambda_{e}, k_{g}, k_{e}\right)$ além de, não ter uma forma analítica amigável, não tem uma forma analítica fechada. As integrais com relação a $k_{e}$ e $k_{g}$ não podem ser resolvidas analiticamente. 
Novamente um método computacional como o $\boldsymbol{A B C}$ é necessário para realizar a inferência desejada. O algoritmo 20 apresenta o $\boldsymbol{A} \boldsymbol{B} \boldsymbol{C}$ configurado para gerar amostras da distribuição a posteriori de $\left(\alpha, p, \lambda_{g}, \lambda_{e}, k_{g}, k_{e}\right)$.

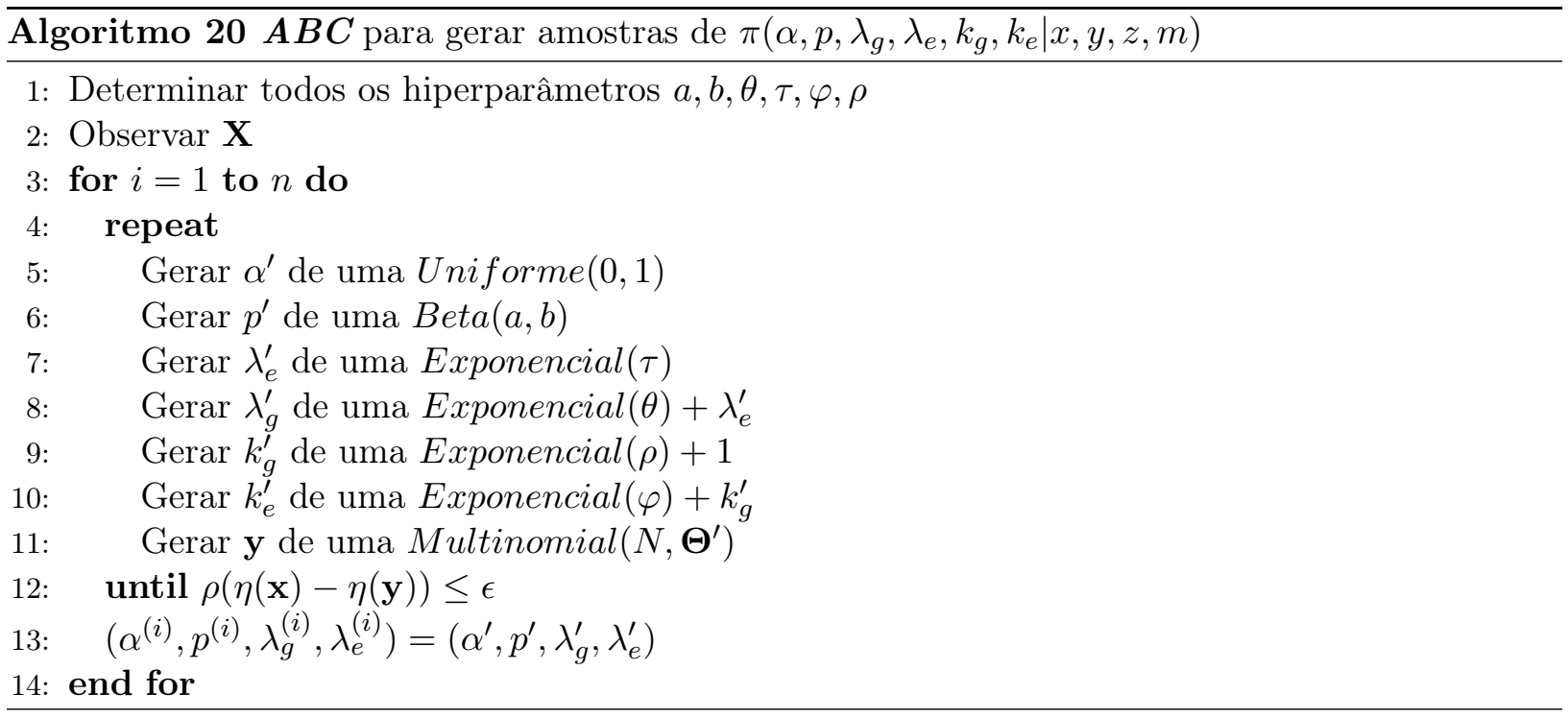

$$
\begin{gathered}
\text { Onde } \Theta^{\prime}=\left(p^{\prime}\left(1-e^{-\left(\frac{T}{\lambda_{e}^{\prime}}\right)^{k_{e}^{\prime}}}\right)\left(1-\alpha^{\prime}\right),\left(1-p^{\prime}\right)\left(1-e^{-\left(\frac{T}{\lambda_{g}^{\prime}}\right)^{k_{g}^{\prime}}}\right)\left(1-\alpha^{\prime}\right), \alpha p\left(1-e^{-\left(\frac{T}{\lambda_{e}}\right)^{k_{e}}}\right)+\right. \\
\left.\alpha^{\prime}\left(1-p^{\prime}\right)\left(1-e^{-\left(\frac{T}{\lambda_{g}^{\prime}}\right)^{k_{g}^{\prime}}}\right), p^{\prime} e^{-\left(\frac{T}{\lambda_{e}^{\prime}}\right)^{k_{e}^{\prime}}}+\left(1-p^{\prime}\right) e^{-\left(\frac{T}{\lambda_{g}^{\prime}}\right)^{k_{g}^{\prime}}}\right) .
\end{gathered}
$$

\subsubsection{Exemplo numérico}

Analogamente à subseção 5.3.1, será usado um exemplo para ilustrar o funcionamento do $\boldsymbol{A B C}$ para realizar a inferência, mas agora com a taxa de falha dos itens crescente. Supõe-se que um certo lote de itens será submetido a metodologia $\boldsymbol{E S S}$, e um especialista do processo de produção dos itens fornece a informação sobre a produção, e do tempo de vida dos itens de baixa e alta qualidade. Com essas informações estabelecem-se as seguintes distribuições a priori:

$$
\begin{aligned}
p & \sim \operatorname{beta}(1,2) ; \\
\lambda_{e} & \sim \operatorname{exponencial}(3) ; \\
\lambda_{g} & \sim \lambda_{e}+\operatorname{exponencial}(2) ; \\
k_{g} & \sim 1+\operatorname{exponencial}(3) ; \\
k_{e} & \sim k_{g}+\operatorname{exponencial}(4) ; \\
\alpha & \sim \operatorname{Uniforme}(0,1) .
\end{aligned}
$$

Com as distribuições de probabilidades a priori já definidas, deve-se encontrar um tempo ótimo, $T$, de duração do estresse. Decidiu-se usar os seguintes custos, $c_{1}=100, c_{2}=20, c_{3}=1, c_{4}=0,01$, iguais ao exemplo anterior. O valor de $R(t, l)$ foi calculado como descrito na seção 5.5.1 para vários valores de $t$. Na figura 5.4 pode ser vista a função de risco a priori para o atual caso em estudo, taxa de falha crescente e o tempo de vida dos itens produzidos seguindo uma distribuição Weibull. Quando minimizada a função exibida na figura 5.4 obtem-se $T=2,38$ horas.

Dos 1000 itens submetidos ao teste de estresse, 160 itens não sobreviveram ao estresse e foram diagnosticados pela autopsia como de baixa qualidade, 330 itens que não sobreviveram ao estresse e foram diagnosticados pela autopsia como de boa qualidade, 493 itens não sobreviveram ao estresse e o processo de autopsia não conseguiu concluir sobre a qualidade destes itens e, 17 itens sobreviveram ao estresse. 
Para a implementação do $\boldsymbol{A} \boldsymbol{B} \boldsymbol{C}$ foram usadas as mesmas funções $\eta(\cdot)$ e $\rho(\cdot)$ para quando a taxa de falha era constante. A figura 5.5 apresenta os histogramas das amostras geradas pelo algoritmo 20 para a distribuição a posteriori de $\left(\alpha, p, \lambda_{g}, \lambda_{e}, k_{g}, k_{e}\right)$. É possível ver que as amostras são coerentes com o que era esperado encontrar. As distribuições a priori de $\alpha$ e $p$ são da família de distribuições Beta, assim como supõe ser sua distribuição a posteriori. As distribuições a priori de $\lambda_{g}, \lambda_{e}, k_{g} \mathrm{e}$ $k_{e}$ são da família de distribuições Exponencial, deslocadas, mas ainda Exponencial. De acordo com os histogramas, as distribuições a posteriori de $\lambda_{g}, \lambda_{e}, k_{g}$ e $k_{e}$ parecem ser distribuições Gama, também deslocadas, o que era esperado.

Ao calcular as estimativas de Bayes, com relação à perda quadrática, para $\left(\alpha, p, \lambda_{g}, \lambda_{e}, k_{g}, k_{e}\right)$, foi obtido o vetor $(0,5021,0,3233,0,8189,0,4795,1,267,1,524)$ como estimativas.

A figura 5.6 apresenta a função de risco a posteriori calculada usando as amostras apresentadas na figura 5.5. Ao manter os custos $c_{1}, c_{2}, c_{3}$ e $c_{4}$ fixos, obtêm-se o instante de tempo $T=4,27$ horas como o que minimiza o risco a posteriori .

\subsection{Previsões de futuros enviromental stress screening}

Seguindo os mesmos passos presentes nos capítulos anteriores, a análise estatística torna-se completa com a predição de novas observações.

Dois novos ensaios de estresse serão realizados, em um é suposto a taxa de falha constante e no outro a taxa é crescente.

Nos dois cenários, taxa de falha constante e crescente, esse novo ensaio de estresse acontecerá com uma duração determinada pelo mínimo da função de risco a posteriori de cada um dos cenários (figuras 5.2 e 5.6), 10,01 horas no cenário da taxa de falha constante 40,27 horas para o cenário da taxa de falha crescente.

Nos dois cenários um lote de 5 itens foi submetido a estresse. O que gera um espaço amostral de 56 combinações de $(x, y, z, m)$ tais que, $x+y+z+m=5$.

Assim como nos capítulos anteriores, a distribuição preditiva será obtida numericamente através do cálculo de

$$
\frac{1}{n} \sum_{i=1}^{n} P_{\mathbf{x}}\left(X=x, Y=y, Z=z, M=m \mid \mathbf{\Theta}_{i}\right),
$$

onde $P_{\mathbf{x}}\left(X=x, Y=y, Z=z, M=m \mid \boldsymbol{\Theta}_{i}\right)$ representa a função de probabilidade de uma distribuição Multinomial $(5, \Theta)$, e o vetor de probabilidade $\Theta$ é da forma já descrita anteriormente, reforçando que o vetor de probabilidade tem formas diferentes para quando é suposto taxa de falha crescente, e taxa de falha constante.

Para a obtenção da distribuição preditiva foram usadas as amostras geradas pelo $\boldsymbol{A} \boldsymbol{B} \boldsymbol{C}$, para os dois casos de taxas de falha.

Por uma impossibilidade física, não foi possível representar graficamente a distribuição preditiva de $(x, y, z, m)$, nas figuras ?? e ?? estão as distribuições marginais de $X, Y, Z$ e $M$. Nas tabelas são apresentadas as distribuições preditivas, conjunta, de $(X, Y, Z, M)$.

\subsection{Considerações finais}

O modelo discutido aqui,assim como o modelo apresentado por Tsunemi et al. (2012) apresentam avanços sofisticados na maneira de abordar cada um dos problemas em questão.O modelo de Barlow et al. (1994) foi pioneiro ao enxergar a metodologia de Enviromental Stress Screening sob a ótica Bayesiana.

Todo esse arrojo teórico cobrou seu preço na forma de complexidade computacional, o que impossibilita a utilização dos modelos em situações não acadêmicas. O modelo discutido nesse capítulo, em particular apresenta uma complexidade computacional tão extrema que nem mesmo o emprego do algoritmo de Metropolis-Hastings conseguiu torna-lo aplicável para um caso não acadêmico.

O uso do $\boldsymbol{A} \boldsymbol{B} \boldsymbol{C}$, na sua forma mais simples, foi essencial para conseguir aplicar o modelo de Barlow et al. (1994) em uma situação que pode ser real, e ainda possibilitou o estudo de uma nova suposição, contemplando a suposição de uma taxa de falha crescente. 
Infelizmente não é possível saber o quanto a amostra gerada pelo $\boldsymbol{A} \boldsymbol{B} \boldsymbol{C}$ se aproxima da distribuição alvo nos exemplos abordados aqui, já que é bastante difícil, e custoso, obter a forma gráfica da distribuição a posteriori. Mas ao utilizar a amostra gerada pelo algoritmo de Metropolis-Hastings como ponto de referência, considerando as devidas proporções, pode-se dizer que a amostra gerada pelo $\boldsymbol{A} \boldsymbol{B C}$ produziu uma amostra com a mesma qualidade que o $\boldsymbol{M C M C}$, com menos com menos custo computacional envolvido, tornando o $\boldsymbol{A B C}$ Mais eficiente que o $\boldsymbol{M C M C}$ para gerar observações das distribuições 5.8 e 5.30 . 

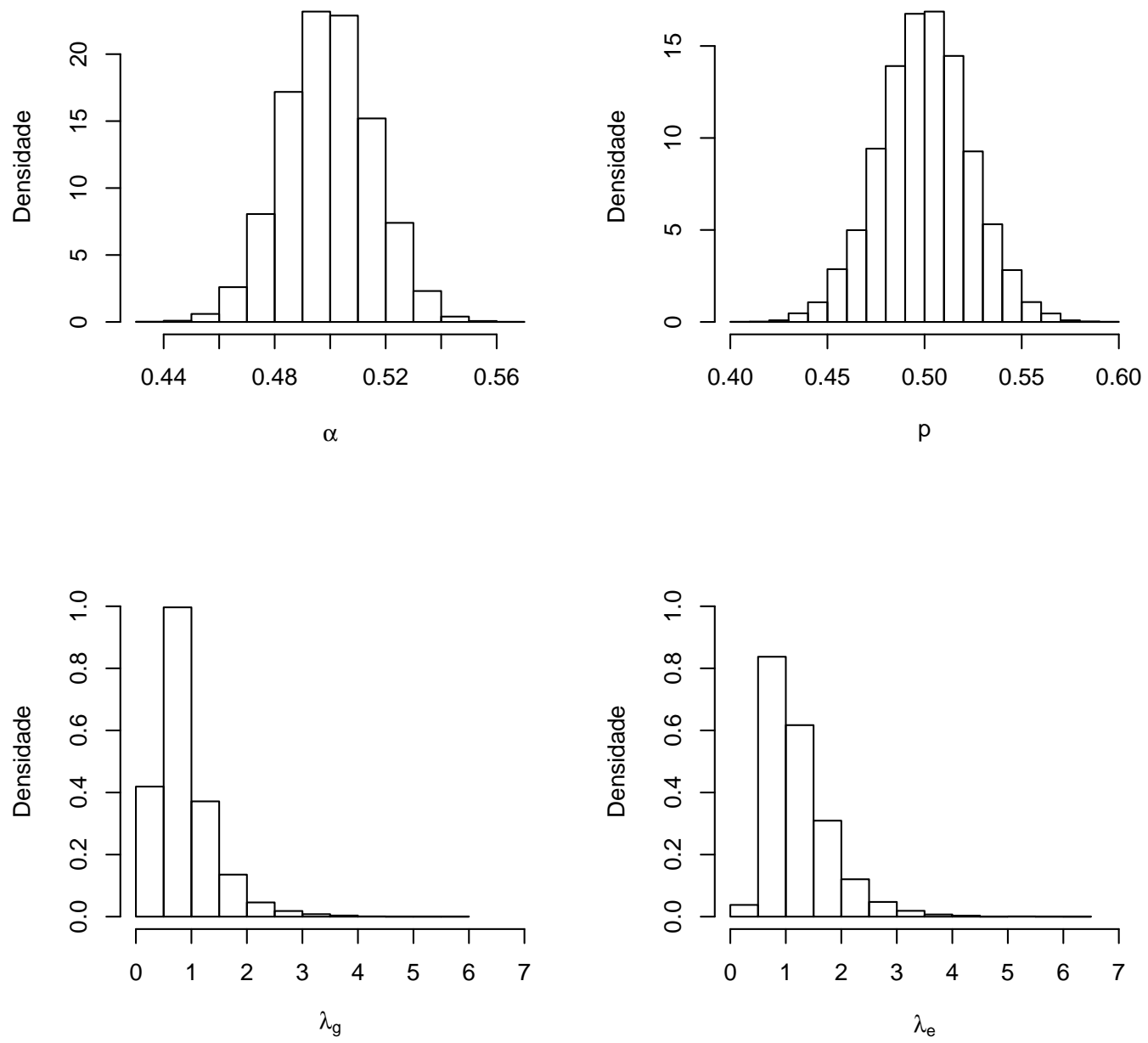

Figura 5.1: Histograma das amostras da distribuição de probabilidade a posteriori de $p, \lambda_{e}$ e $\lambda_{g}$ geradas pelo algoritmo 18 


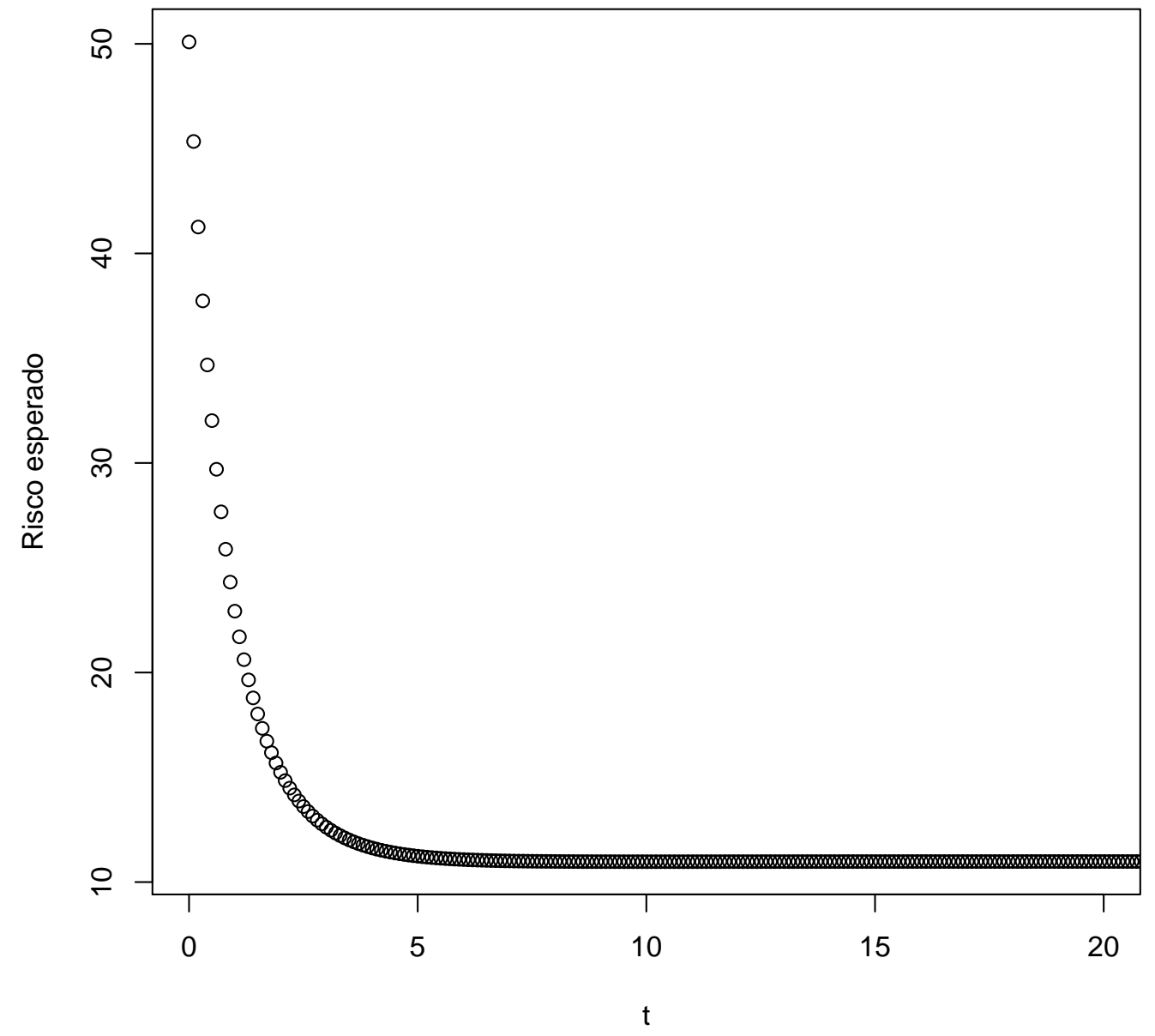

Figura 5.2: Função de risco a posteriori 

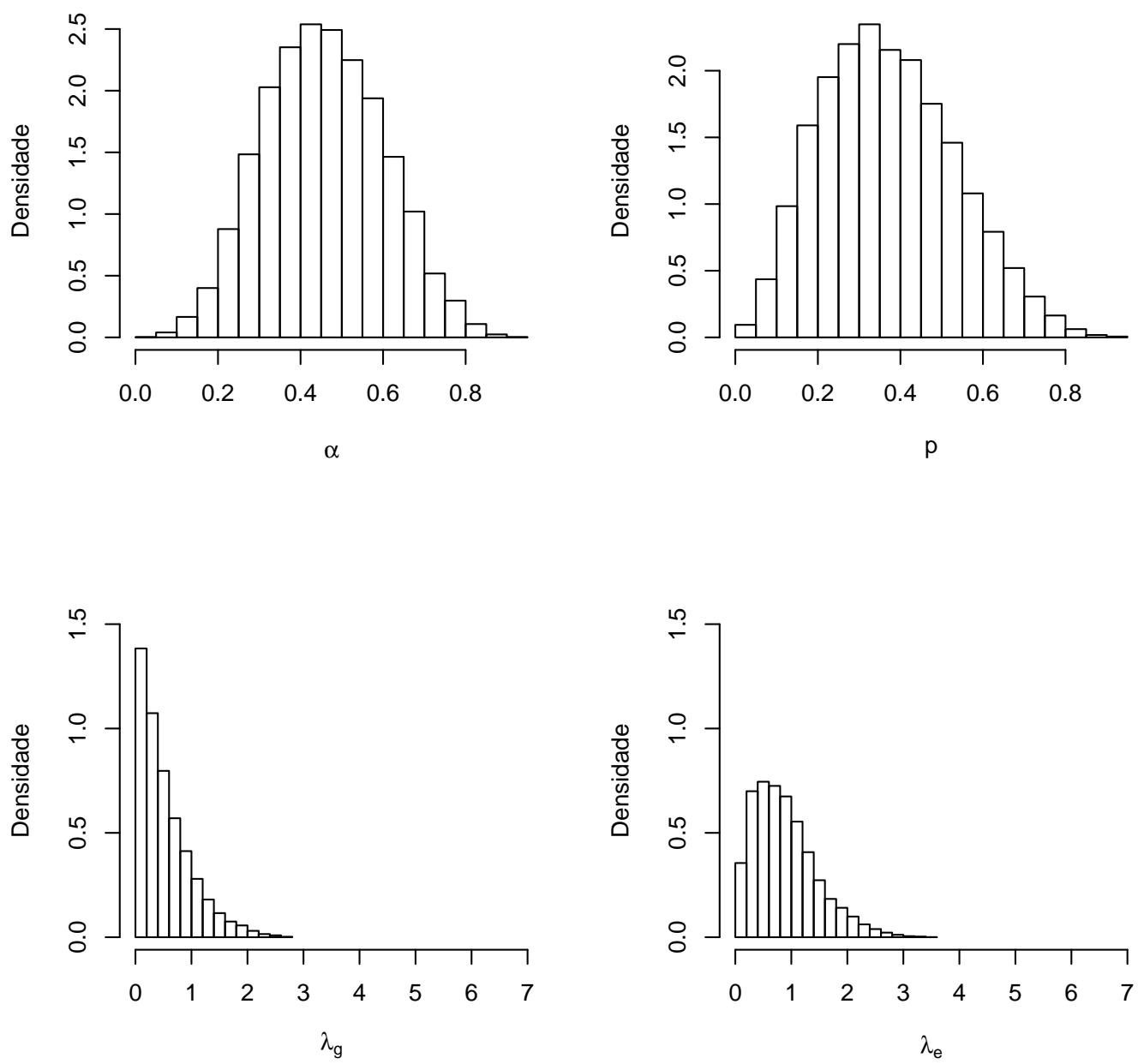

Figura 5.3: Histograma das amostras de $\left(\alpha, p, \lambda_{g}, \lambda_{e}\right)$ gerados pelo $M C M C$, algoritmo 19 


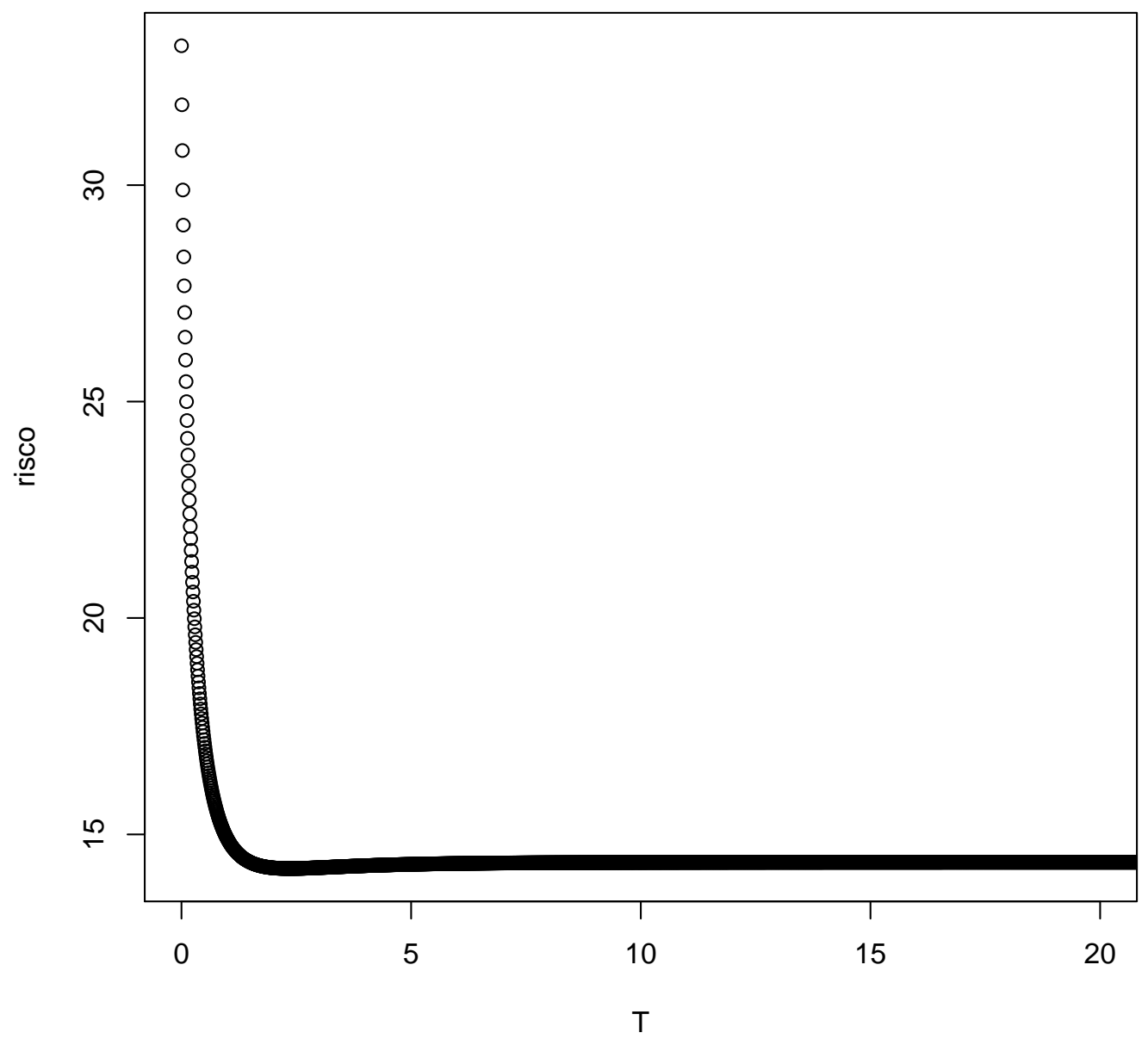

Figura 5.4: Função de risco a priori quando a taxa de falha é crescente 

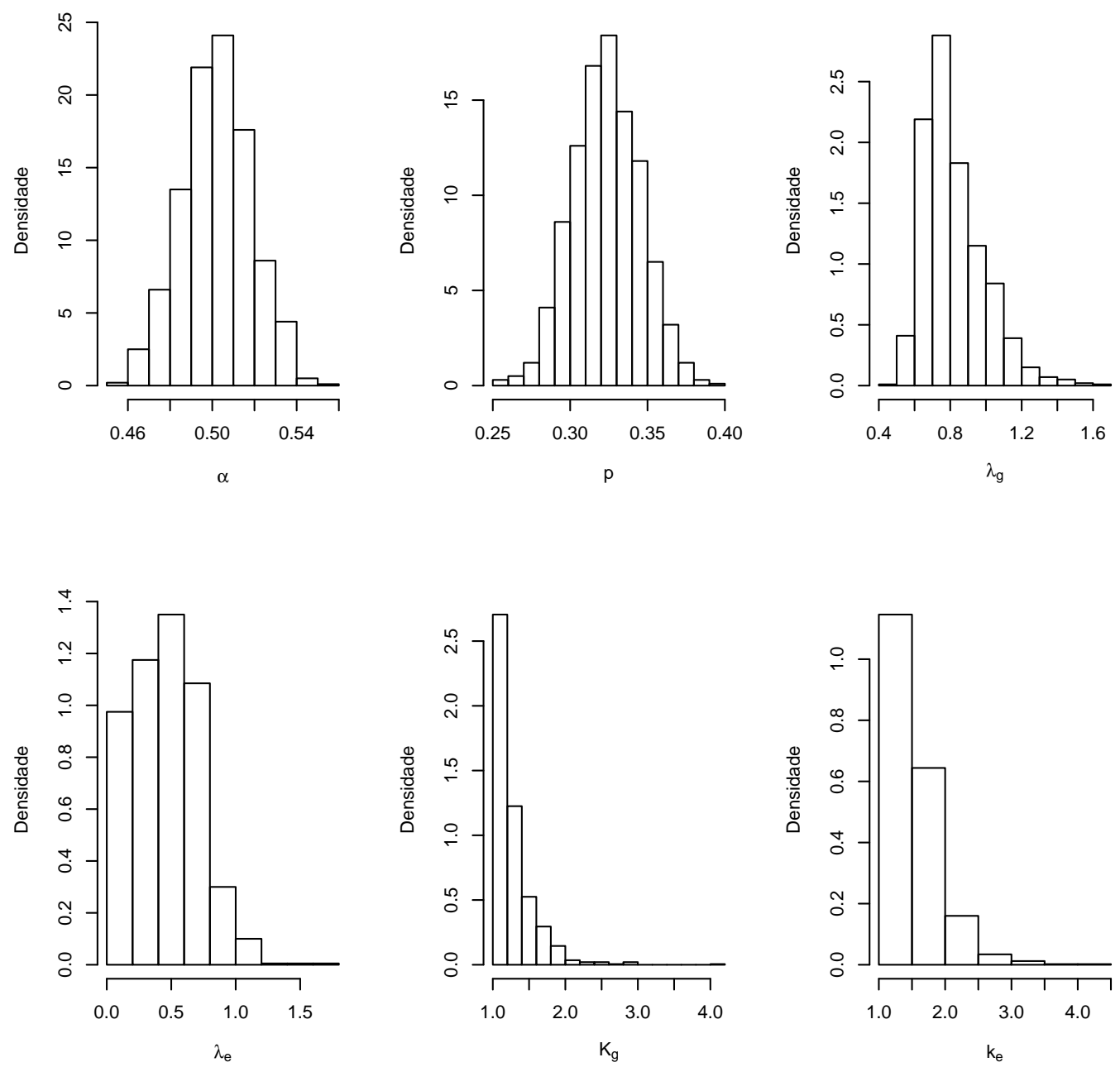

Figura 5.5: Histograma das amostras da distribuição a posteriori de $\left(\alpha, p, \lambda_{g}, \lambda_{e}, k_{g}, k_{e}\right)$ 


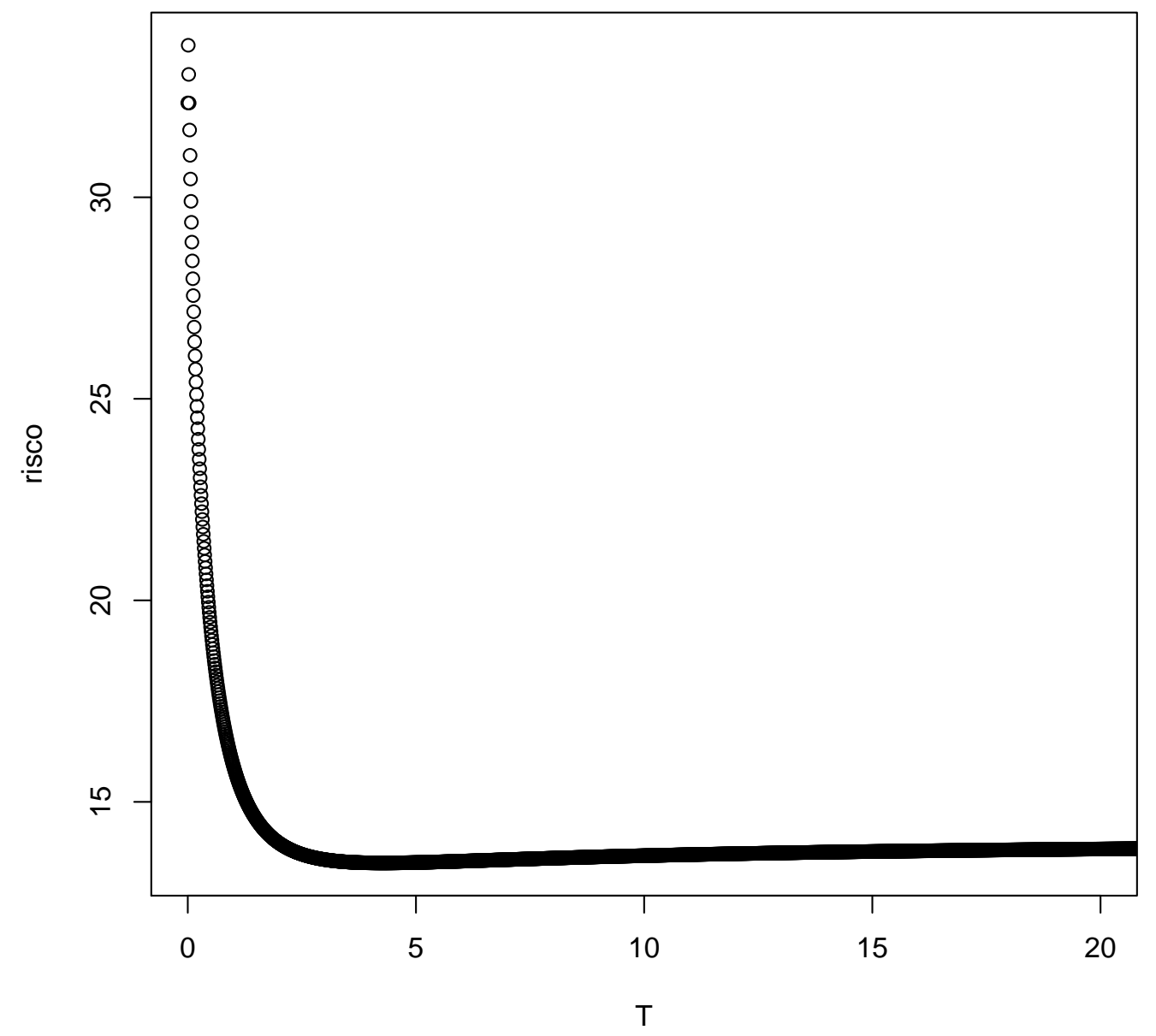




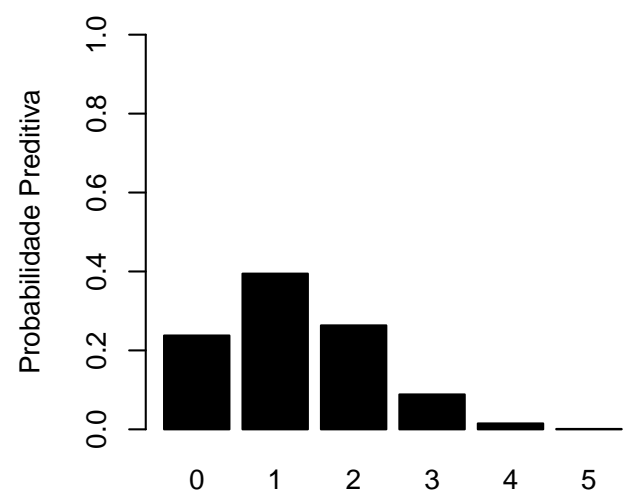

Número de itens que falharam e são de baixa qualidaı

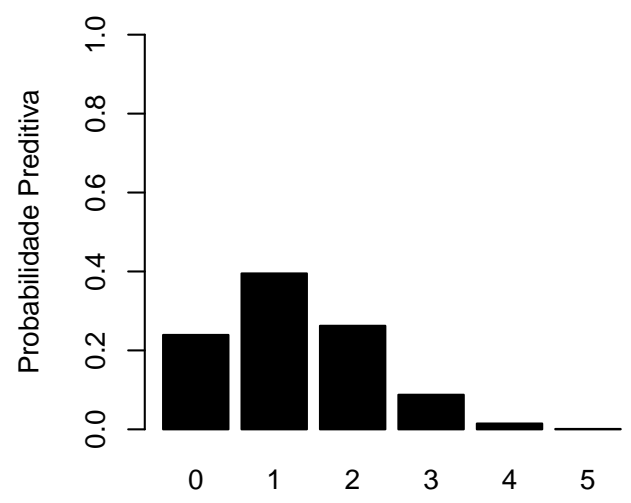

Número de itens que falharam e são de boa qualidad

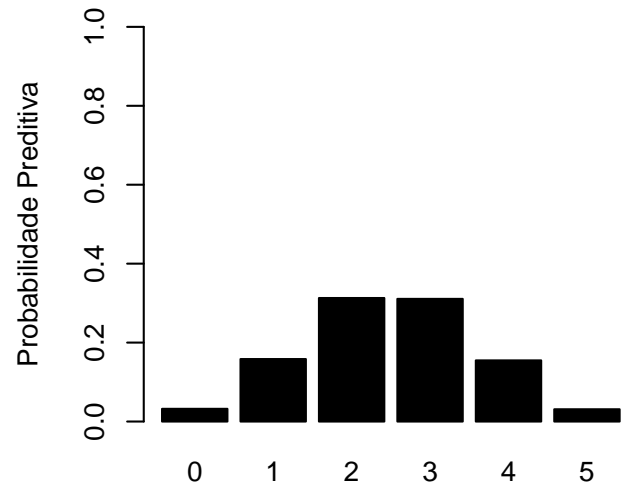

itens que falharam e que não é sabido se são de boa ou

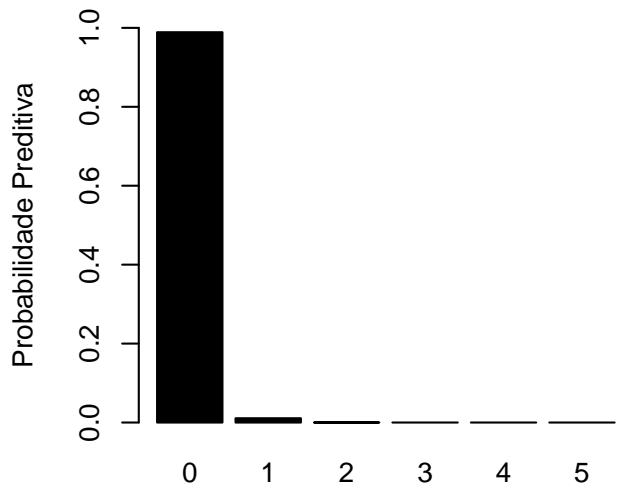

Número de itens que sobreviveram ao estresse

Figura 5.7: Gráfico de barras das distribuições preditivas marginais de $x, y, z$ e m suponto taxa de falha constante. 


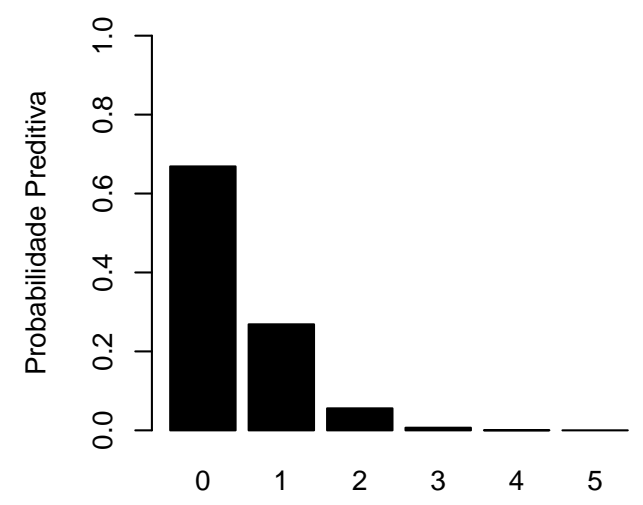

Número de itens que falharam e são de baixa qualida

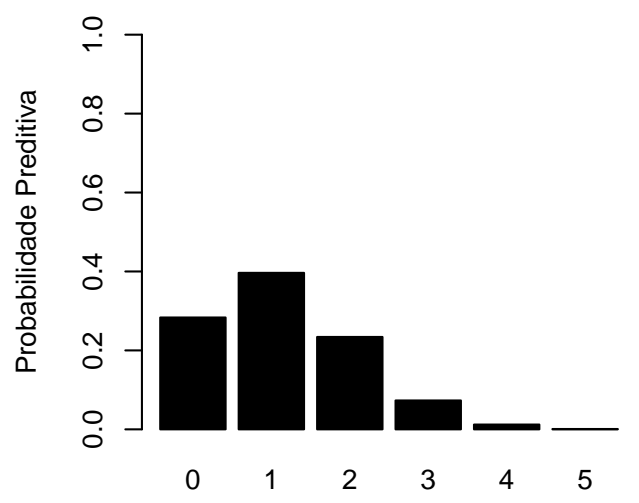

Número de itens que falharam e são de boa qualidad

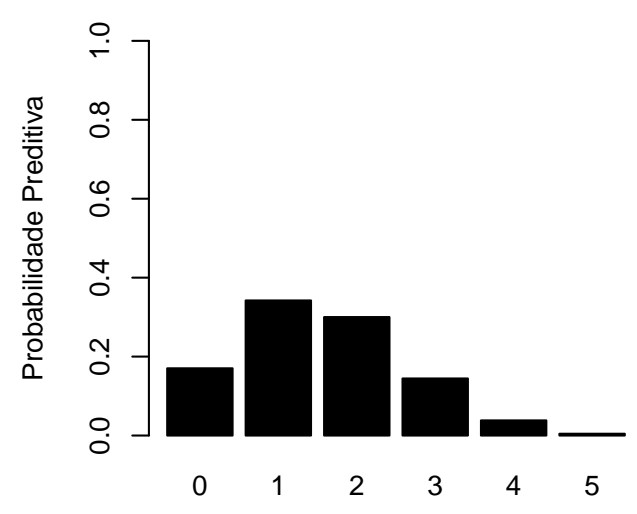

itens que falharam e que não é sabido se são de boa ou

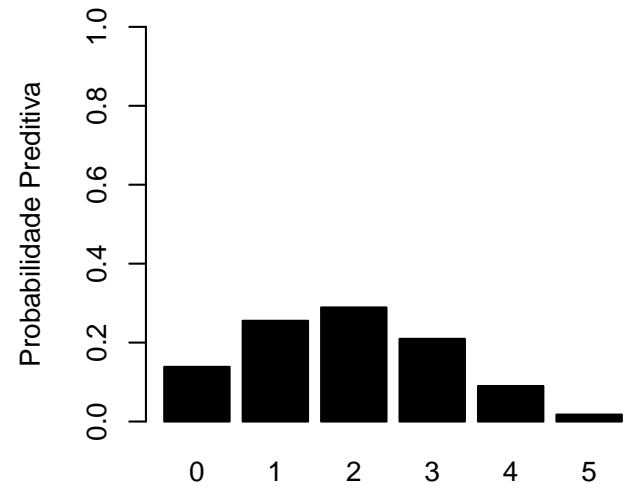

Número de itens que sobreviveram ao estresse

Figura 5.8: Gráfico de barras das distribuições preditivas marginais de $x, y, z$ e $m$ suponto taxa de falha crescente. 


\section{Capítulo 6}

\section{Conclusões}

Após exibir e discutir os modelos de Tsunemi et al. (2012) e Barlow et al. (1994), percebe-se, sem sombra de duvidas, que ambos são extremamente sofisticados. Em Tsunemi et al. (2012) as suposições teóricas chegam ao ponto de admitir uma massa de probabilidade para o infinito, fato bastante raro, mas que faz todo sentido no problema estudado e na forma de como ele é abordado. O modelo não-paramétrico é o que melhor se utiliza de ferramentas teóricas para modelar o problema, mas como já foi comentado, todo esse ferramental teórico, deixou o modelo inutilizável para situações não acadêmicas. É plausível falar que o fator que mais contribui com a dificuldade de implementar o modelo são os conjuntos $\mathbb{C}_{k}$ e $\mathbb{C}_{k}^{\prime}$, que crescem de tamanho de uma maneira incrivelmente rápida a medida que $\tau$ cresce, esse fato impossibilitou até a utilização do amostrador de Gibbs.

O $\boldsymbol{A B C}$ pode não ter se mostrado um bom método para gerar amostras, e por consequência uma boa estimativa, para o modelo apresentado em Tsunemi et al. (2012), já que superestimou a proporção de itens não-conformes. Mas foi o único, dos métodos comparados, que conseguiu gerar amostras para valores de $\tau$ maiores que um, lembrando que em seu trabalho Tsunemi et al. (2012) conseguem obter estimativas da proporção de itens não-conformes para quando se detecta um item não conforme na sexta inspeção, no capítulo 4 encontram-se estimativas para $\tau$ indo de 1 até 10 . Com isso surge um questionamento: vale a pena utilizar um método que sabidamente superestima a proporção de itens não-conformes, mas consegue estimar essa proporção para situações não acadêmicas? Se não, então como obter as estimativas, se o estimador de Tsunemi et al. (2012) demanda uma quantidade extremamente enorme de recursos?

O modelo de Barlow et al. (1994), pioneiro na abordagem Bayesiana à metodologia de Enviromental Stress Screening, ao trazer o uso das distribuições a priori para a análise do problema em questão, além de uma maior exatidão teórica à análise, origina um modelo bastante complicado, com seis somatórios encaixados, onde o limite de cada somatório depende diretamente dos dados observados. Como falado no capítulo 5, os somatórios encaixados geram uma quantidade exacerbadamente grande de parcelas. No exemplo acadêmico com um lote de 10 itens submetidos ao estresse, resultaram 240 parcelas a serem calculadas, e depois somadas, para realizar a análise dos dados observados, de acordo com o modelo de Barlow et al. (1994), exemplos onde lotes maiores são observados são impraticáveis.

O algoritmo de Metropolis-Hastings só pode ser aplicável no modelo de Barlow et al. (1994) em exemplo como o do lote de 10 itens, ou seja, não é possível utilizar este modelo para situações não acadêmicas, mesmo com a utilização de um algoritmo $M C M C$. Isto se deve à necessidade do uso, direta ou indiretamente, da função de verossimilhança. Realizar cálculos nessa função, no modelo em questão, é extremamente custoso, talvez tão custoso quanto no modelo estudado no capítulo 4.

Como o $\boldsymbol{A B C}$ não realiza cálculos na função de verosimilhança, fato discutido no capítulo 2, tornou-se o candidato ideal para auxiliar o processo inferencial do modelo de Barlow et al. (1994), revelando-se extremamente eficiente, e necessário. Sem o $\boldsymbol{A B C}$ não haveria como realizar a inferência do modelo de estudo, para o caso da taxa de falhas constante, nem seria possível estender o modelo, quando é considerada a suposição de que a taxa de falha do produto aumenta com o tempo.

Em suma, nos dois trabalhos, o $\boldsymbol{A} \boldsymbol{B} \boldsymbol{C}$ se mostrou uma ferramenta bastante útil, imprescindível dependendo da situação, para que fosse possível estudá-los, analisá-los e evolui-los. 


\subsection{Sugestões para Pesquisas Futuras}

Ainda há trabalho para ser feito. A utilização do $\boldsymbol{A} \boldsymbol{B} \boldsymbol{C}$ no modelo de Tsunemi et al. (2012) ainda não está totalmente satisfatória. Estudar o comportamento das estimativas deste modelo, geradas com auxílio do $\boldsymbol{A} B \boldsymbol{C}$, utilizando outras variações do $\boldsymbol{A} \boldsymbol{B C}$ é um objetivo para um trabalho futuro, como comentado em Marin et al. (2011), a variante $\boldsymbol{M} \boldsymbol{C} \boldsymbol{M} \boldsymbol{C}-\boldsymbol{A} \boldsymbol{B} \boldsymbol{C}$ é uma boa candidata para tal tarefa.

Uma outra sugestão, mas agora para o modelo de Barlow et al. (1994), seria estipular varias categorias de qualidade diferentes, aumentando assim a dimensão da Multinomial, utilizada como distribuição dos dados. Também pode-se experimentar diferentes taxas de falha, inclusive uma que seja decrescente.

É considerado válido o esforço de aplicar o $\boldsymbol{A} \boldsymbol{B C}$ a qualquer modelo estatístico/probabilístico que seja analiticamente complicado, um exemplo é o modelo de três parâmetros da TRI, Teoria da Resposta ao Item. 


\section{Referências Bibliográficas}

Arruda (2000) Marcelo Leme de Arruda. Poisson, Bayes, Futebol e DeFinetti. Dissertação de mestrado, Instituto de Matemática E Estatística da Universidade de São Paulo. Citado na pág. 23, 28,31

Barlow et al. (1994) Richard E. Barlow, Carlos Aalberto B. Pereira e Sergio Wechsler. A bayesian approach to enviromental stress screening. Naval Research Logistics, 41:215-228. Citado na pág. 1, $2,67,68,69,70,71,72,73,74,77,87,88$

Bayarri e DeGroot (1992) M. Bayarri e M. DeGroot. Difficulties and ambiguities in the definition of a likelihood function. Statistical Methods EGamp; Applications, 1(1):1-15. Citado na pág. 6, 7

Beaumont (2010) Mark A. Beaumont. Approximate bayesian computation in evolution and ecology. Annual Review of Ecology, Evolution, and Systematics, 41(1):379-406. Citado na pág. 2

Beaumont et al. (2002) Mark A. Beaumont, Wenyang Zhang e David J. Balding. Approximate bayesian computation in population genetics. Genetics, 162(4):2025-2035. Citado na pág. 2

Blum et al. (2013) M. G. B. Blum, M. A. Nunes, D. Prangle e S. A. Sisson. A comparative review of dimension reduction methods in approximate bayesian computation. Statist. Sci., 28 (2):189-208. doi: 10.1214/12-STS406. URL http://dx.doi.org/10.1214/12-STS406. Citado na pág. 9

Calle e Gómez (2001) M. Luz Calle e Guadalupe Gómez. Nonparametric bayesian estimation from interval-censored data using monte carlo methods. Journal of Statistical Planning and Inference, 98(1-2):73-87. Citado na pág. 54

Campos e Wechsler (2012) Thiago F. Campos e Sergio Wechsler. Abc for kids. Em XI BRAZILIAN MEETING ON BAYESIAN STATISTICS: EBEB 2012, volume 1490, páginas 67-74. Citado na pág. 14

Casella e George (1992) George Casella e Edward I George. Explaining the gibbs sampler. The American Statistician, 46:167-174. Citado na pág. 5, 14, 24

Chire (2013) Verûnica Amparo Quispe Chire. Infêrencia em DIstribuições Discretas Bivariadas. Dissertação de mestrado, Universidade Federal de São Carlos. Citado na pág. 21, 22, 23, 25, 29, 30

Cornuet et al. (2008) Jean-Marie Cornuet, Filipe Santos, Mark A. Beaumont, Christian P. Robert, Jean-Michel Marin, David J. Balding, Thomas Guillemaud e Arnaud Estoup. Inferring population history with diy abc: a user-friendly approach to approximate bayesian computation. Bioinformatics, 24(23):2713-2719. Citado na pág. 2

Csilléry et al. (2010) Katalin Csilléry, Michael G. B. Blum, Oscar E. Gaggiotti e Olivier François. Approximate Bayesian Computation $(\mathrm{ABC})$ in practice. Trends in Ecology $\&$ Evolution, páginas 1-9. doi: 10.1016/j.tree.2010.04.001. URL http://linkinghub.elsevier.com/retrieve/pii/ S0169534710000662. Citado na pág. 9 
Dean et al. (2014) Thomas A. Dean, Sumeetpal S. Singh, Ajay Jasra e Gareth W. Peters. Parameter estimation for hidden markov models with intractable likelihoods. Scandinavian Journal of Statistics, 41(4):970-987. ISSN 1467-9469. doi: 10.1111/sjos.12077. URL http://dx.doi.org/10.1111/sjos.12077. Citado na pág. 9

DeGroot (1989) Morris H. DeGroot. Probability and Statistics. Addison-Wesley, Reading, Massachusetts. Citado na pág. 5

Ferguson (1973) Thomas S. Ferguson. A Bayesian Analysis of Some Nonparametric Problems. The Annals of Statistics, 1(2):209-230. Citado na pág. 48, 51

Fisher (1922) Ronald A. Fisher. On the mathematical foundations of theoretical statistics. Philosophical Transactions of the Royal Society, 222:309-386. Citado na pág. 5

Gamerman e Lopes (2006) Dani Gamerman e Hedibert Freitas Lopes. Markov Chain Monte Carlo Stochastic Simularion for Bayesian Inference. Chapman \& Hall/CRC. Citado na pág. 5, 11, $21,25,26$

Holgate (1964) P. Holgate. Estimation for the bivariate poisson distribution. Biometrika, 51(1-2): 241-245. Citado na pág. 22

Marin et al. (2011) Jean-Michel Marin, Pierre Pudio, Christian P. Robert e Rubin J. Ryder. Approximate bayesian computational methods. Statistics and Computing, 21(2):289-291. Citado na pág. $7,10,14,88$

Marjoram et al. (2003) Paul Marjoram, John Molitor e Vincent Plagnol. Markov chain monte carlo without likelihoods. Proceedings of the National Academy of Sciences, 100(26):15324-15328. Citado na pág. 10

Pritchard et al. (1999) J. Pritchard, M. Seielstad e M. Perez-Lezaun, A. Feldman. Population growth of human y chromosomes: a study of y chromosome microsatellites. Molecular Biology and Evolution, 16:1791-1798. Citado na pág. 8

Rubin (1984) D. Rubin. Bayesianly justifiable and relevant frequency calculation fot the applied statistician. Annals of Statistics, 12(4):1151-1172. Citado na pág. 7, 8

Sethuraman (1994) Jayaram Sethuraman. A constructive definition of Dirichlet priors. Statistica Sinica, 4:639-650. Citado na pág. 48

Sisson et al. (2007) S. A. Sisson, Y. Fan e Mark M. Tanaka. Sequential monte carlo without likelihoods. Proceedings of the National Academy of Sciences, 104(6):1760-1765. doi: 10.1073/ pnas.0607208104. URL http://www.pnas.org/content/104/6/1760.abstract. Citado na pág. 9

Stern e Zacks (2002) Júlio M. Stern e Shelly Zacks. Testing the independence of poisson variates under the holgate bivariate distribution : the power of a new evidence test. Relatório Técnico RT-MAC-2002-03, Universidade de São Paulo (São Paulo, BR), São Paulo. URL http://opac. inria.fr $/$ record=b1044879. Citado na pág. 21

Taguchi et al. (1989) Genichi Taguchi, Elsayed A. Elsayed e Thomas C. Hsiang. Quality Engineering in Production Systems. McGraw-Hill. Citado na pág. 1, 45

Tsunemi (2009) Miriam H. Tsunemi. Um modelo Bayesiano semi-parametrico para o monitoramento "on-line" de qualidade de Taguchi para atributos. Tese de doutorado, Instituto de Matemática e Estatística da Universidade de São Paulo. Citado na pág. 54, 57

Tsunemi et al. (2012) Miriam H. Tsunemi, Thiago F. Campos, Luís G. Esteves, José G. Leite e Sergio Wechsler. A bayesian nonparametric model for taguchi's on-line quality monitoring procedure for attributes. Journal of Statistical Planning and Inference, 142(9):2701-2709. Citado na pág. $1,2,45,46,47,48,51,52,53,54,55,56,57,77,87,88$ 
Wilkinson (2008) R. D. Wilkinson. Approximate Bayesian computation (ABC) gives exact results under the assumption of model error. ArXiv e-prints. URL http://adsabs.harvard.edu/abs/ 2008arXiv0811.3355W. Citado na pág. 10

Zarepour e Labadi (2011) Mahmoud Zarepour e Luai Al Labadi. On a rapid simulation of the dirichlet process. http://arxiv.org/abs/110\%.0521v2. Citado na pág. 48, 51, 54 